\title{
Advancing Forensic DNA Processing with Optical Detection and Microfluidic Technologies
}

\author{
Kimberly Renee Jackson \\ Virginia Beach, Virginia
}

B.S. Chemistry, Virginia Polytechnic Institute and State University, 2011

\begin{abstract}
A Dissertation presented to the Graduate Faculty of the University of Virginia in Candidacy for the Degree of

Doctor of Philosophy
\end{abstract}

Department of Chemistry

University of Virginia

August 2016 


\begin{abstract}
DNA forensic technology capabilities continue to increase with growing demands for automated, accurate, and rapid processing methods. Unfortunately, automation is commonly associated with complex and expensive instruments, and is not a viable solution for low-resource laboratories. In an effort to keep current DNA processing simple and cost-effective, this work utilizes microfluidic technology and optical detection for rapid screening and purification of forensically-relevant samples.
\end{abstract}

Fast DNA screening methods are necessary to provide contextual clues for criminal cases, and determine which forensic samples are to be further processed. Two inexpensive optical methods are introduced for sequence-specific detection of nucleic acids using a single temperature amplification method known as loop-mediated isothermal amplification (LAMP). The first method exploits the high affinity between biotin-labeled LAMP amplicons and streptavidin beads for nucleic acid detection. When a target sequence is present, the biotin amplicons tether the streptavidin beads together, resulting in a sequence-specific bead aggregation response that is optically detectable down to single copies of DNA or RNA. A second optical detection method utilized LAMP with an embedded metal-indicator dye to colorimetrically detect fluid-specific mRNA markers for a panel of 5 body fluids. An optimized universal sample procedure allowed for the identification of any combination of the targeted body fluids in up to 23 samples simultaneously using a smart phone camera.

DNA extraction is a critical step in DNA processing following DNA screening that is largely dominated by expensive biorobotic instruments. An affordable handheld centrifugal system and disposable polyethylene terephthalate (Pe) microdevices were developed for costeffective sample lysis and DNA purification. Pe is amendable to simple and rapid fabrication of a 
four-layer extraction device with on-board passive valving capability for increased fluidic control. Using an optimized extraction procedure, DNA was purified from whole blood samples and buccal swab lysates, which yielded strong short tandem repeat (STR) profiles. A lysis domain, specifically for buccal swab cuttings, was integrated with the extraction process to provide sampleto-PCR ready DNA within 30 minutes. Overall this work provides development towards costeffective and rapid DNA processing methods that are amendable to automation and beneficial to all forensic laboratories. 


\section{TABLE OF CONTENTS}

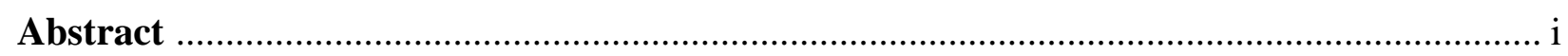

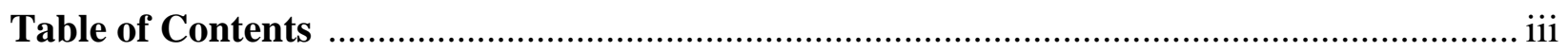

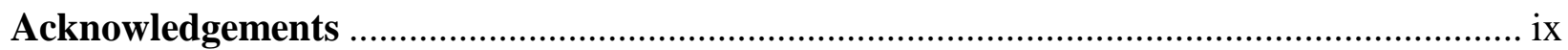

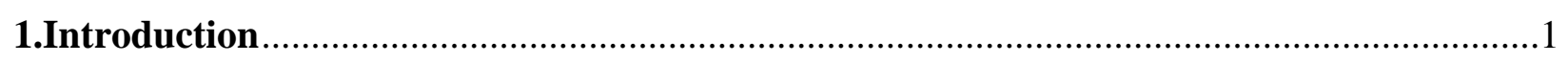

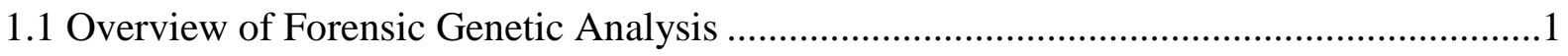

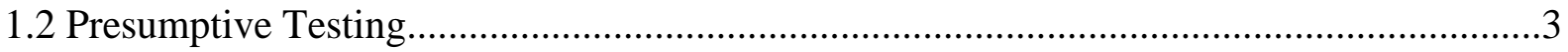

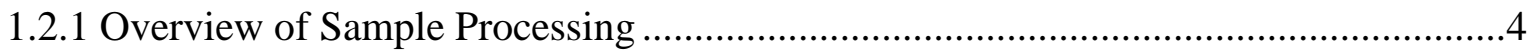

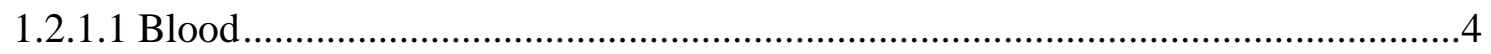

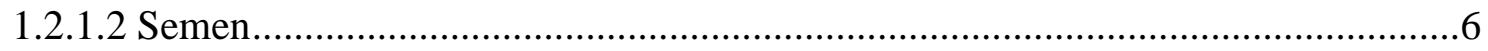

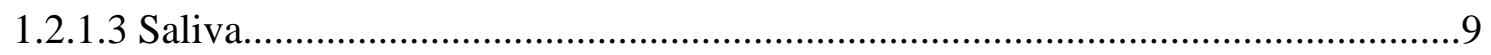

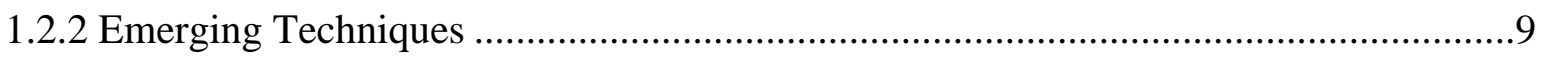

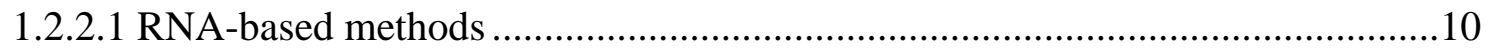

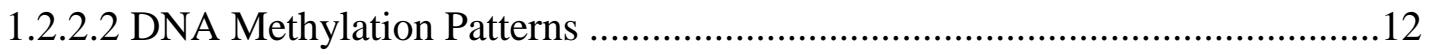

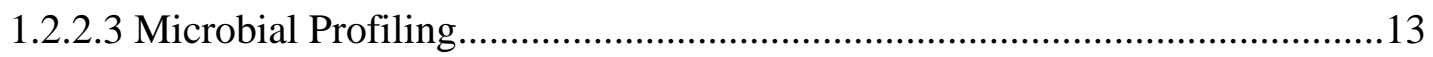

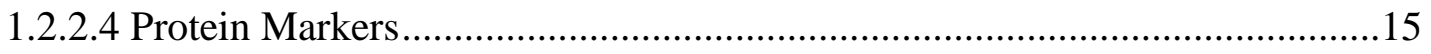

1.3 Conventional Lysis and DNA Purification ........................................................ 18

1.4 DNA Quantitation and Amplification.....................................................................21 
1.5 DNA Separation and Detection ........................................................................24

1.6 Integrated Human Identification Systems .................................................................26

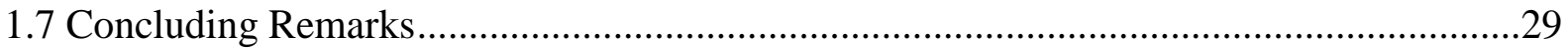

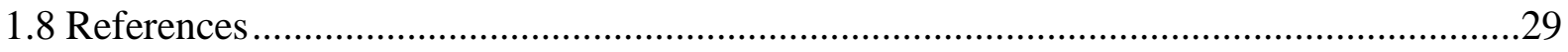

2. Characterization of High Affinity Aggregation for Detection of Targeted Amplicons.....37

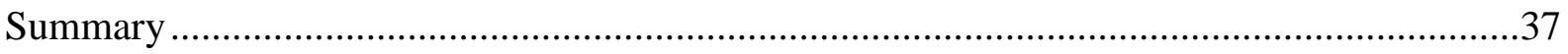

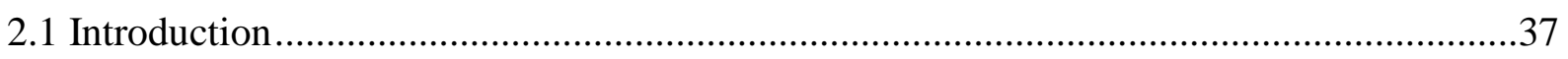

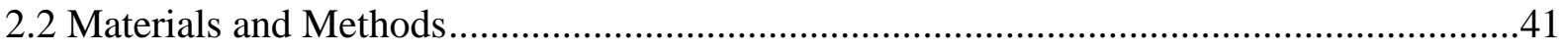

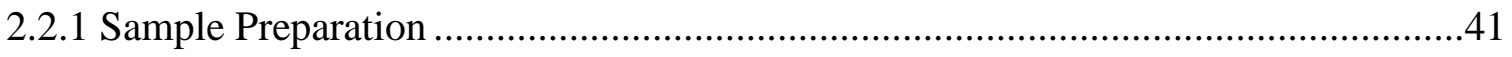

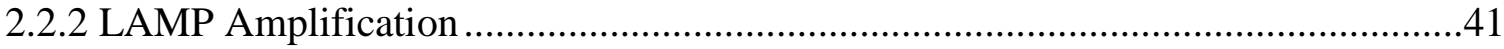

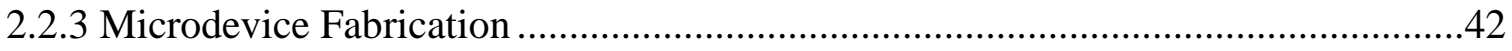

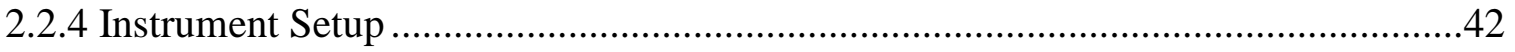

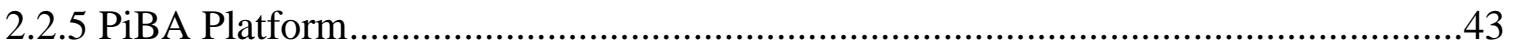

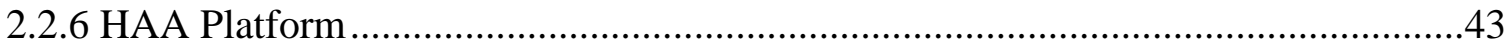

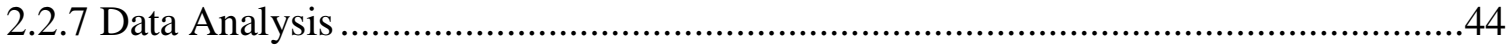

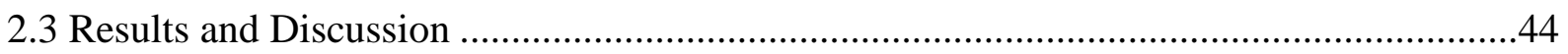

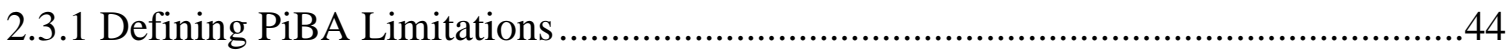

2.3.2 Biotin Labeling during LAMP for HAA ...................................................4

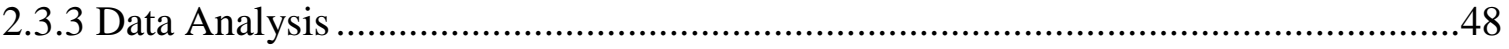

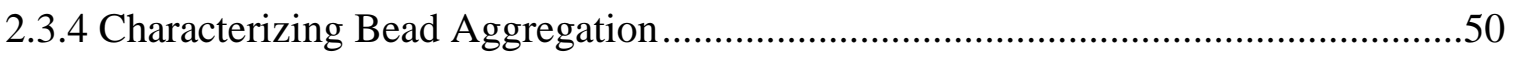

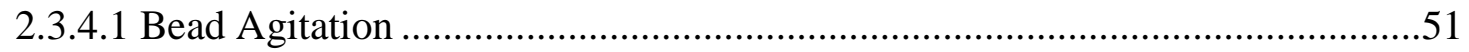

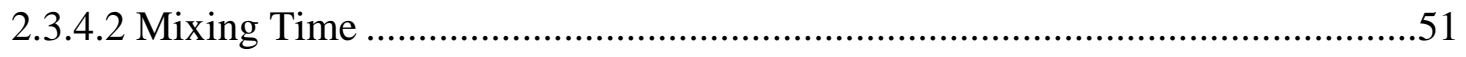




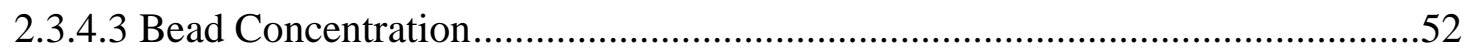

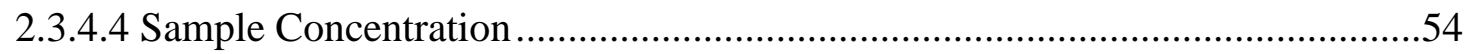

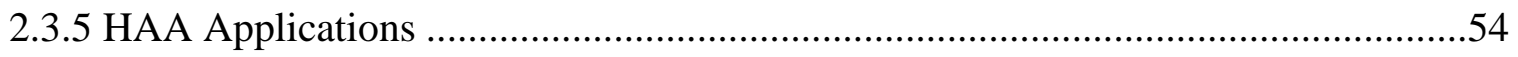

2.3.6 Exploration of HAA Limitations ...........................................................................56

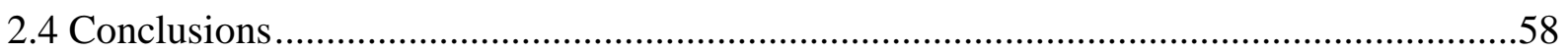

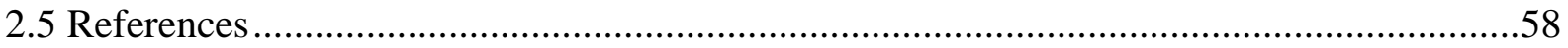

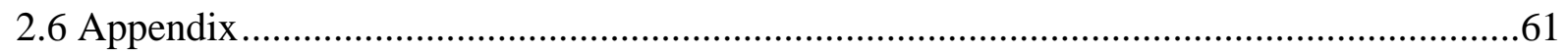

\section{Development of a Novel Loop-Mediated Isothermal}

Amplification Panel for Body Fluid Identification ....................................................62

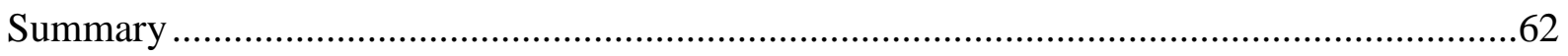

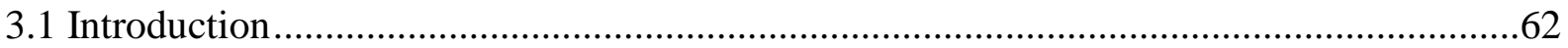

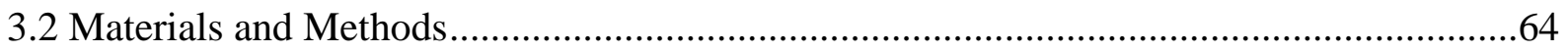

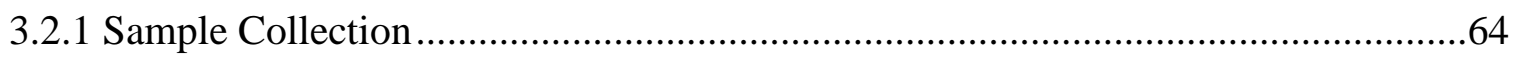

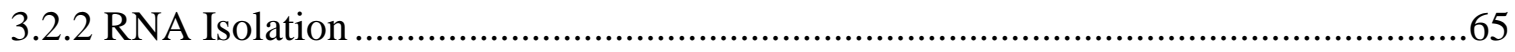

3.2.3 Messenger RNA Marker Selection and LAMP Optimization ..................................66

3.2.4 Colorimetric LAMP Analysis .............................................................................67

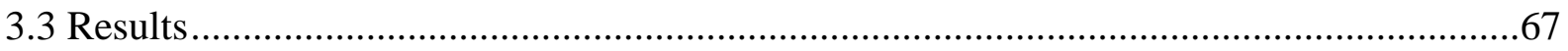

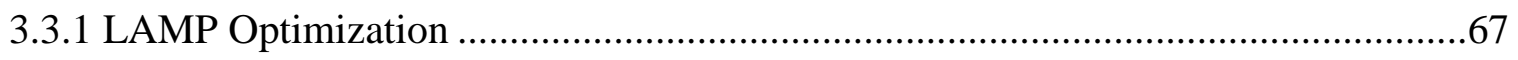

3.3.2 Universal Lysing Procedure ..............................................................................

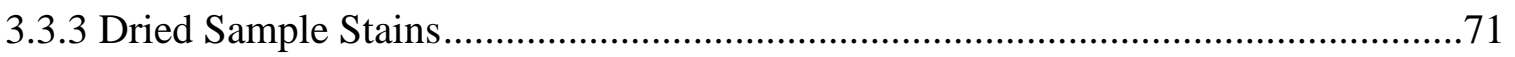

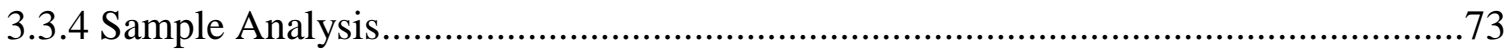




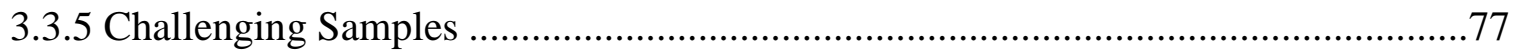

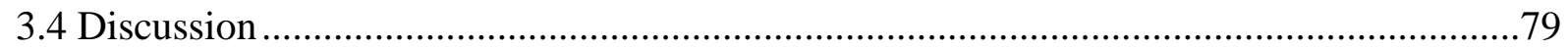

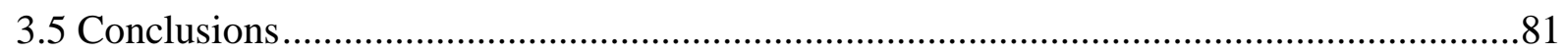

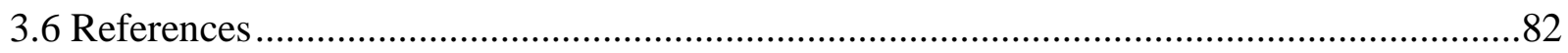

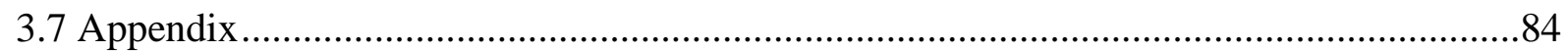

4. DNA Purification using Dynamic Solid-Phase Extraction on a Rotationally-driven

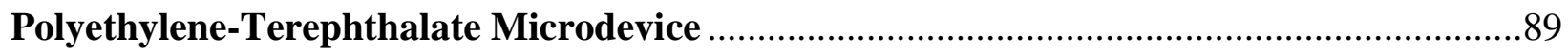

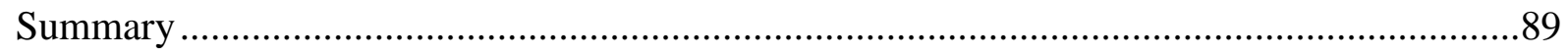

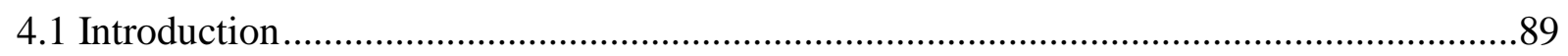

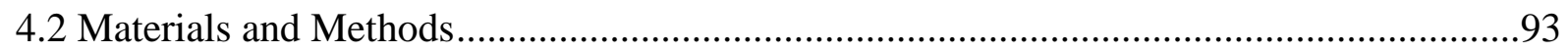

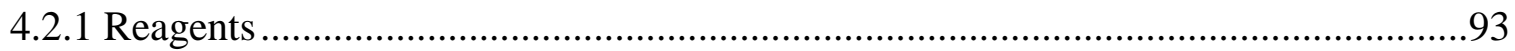

4.2.2 Device Design and Fabrication...........................................................................93

4.2.3 Construction of a Spin Platform and Real-time Monitoring ...................................94

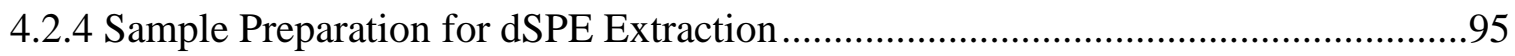

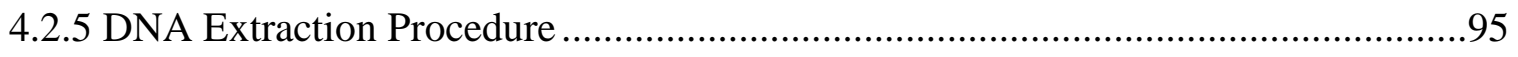

4.2.6 Conventional Solid Phase Extraction......................................................................96

4.2.7 DNA Quantitation and Amplification Procedures ....................................................96

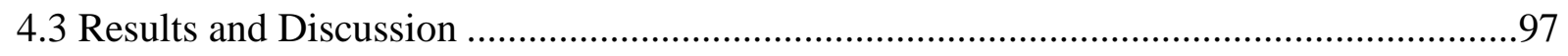

4.3.1 Microdevice Design ..........................................................................................97

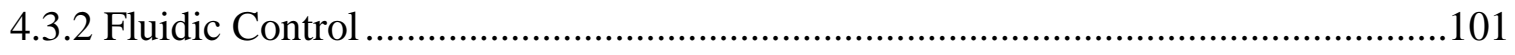

4.3.3 Optimization of the Spin Frequency Protocol for dSPE of DNA .............................104 


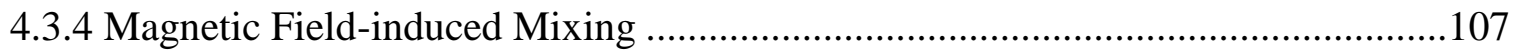

4.3.5 Validation of On-chip Dynamic Solid-Phase Extraction ....................................110

4.3.6 PCR Amplification of Spin-dSPE Purified DNA ...........................................111

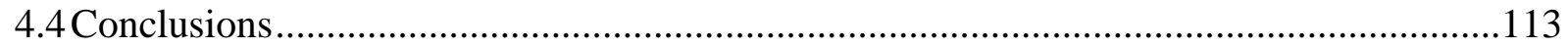

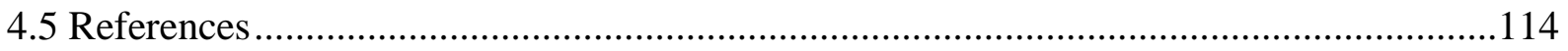

\section{Integrated DNA Lysis and Purification of Buccal Swab DNA on a}

Rotationally-driven Polyethylene-Terephthalate Microdevice .................................118

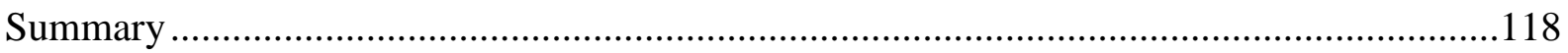

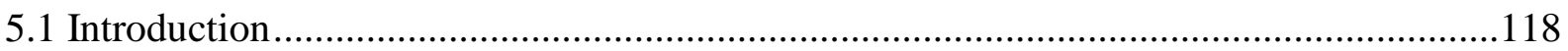

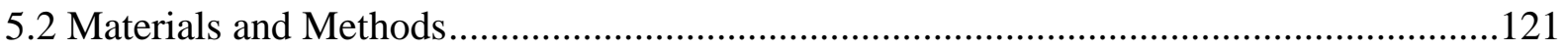

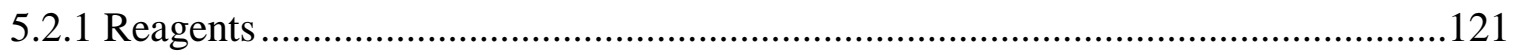

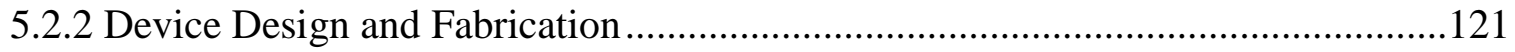

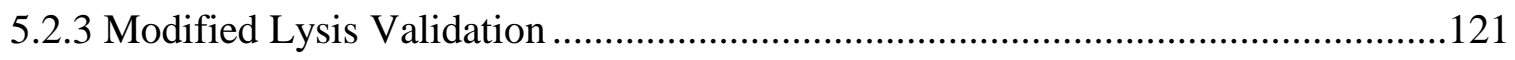

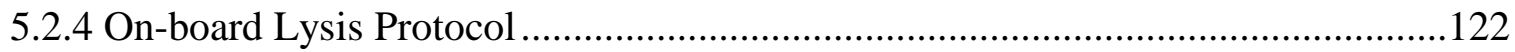

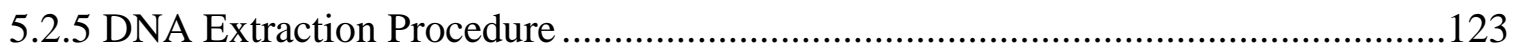

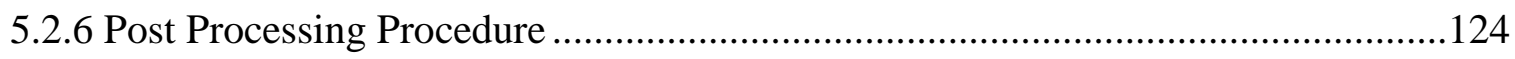

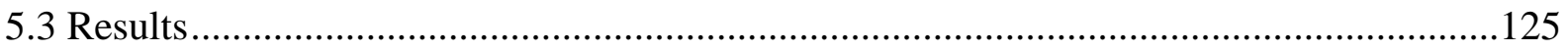

5.3.1 Microdevice Extraction Design Improvements ............................................125

5.3.2 Optimization of the On-chip dSPE Protocol..................................................127

5.3.3 Comparing DNA Extraction Methods ........................................................129

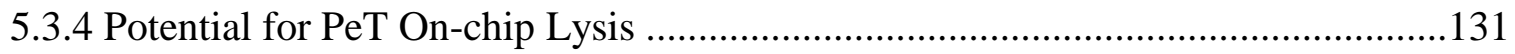




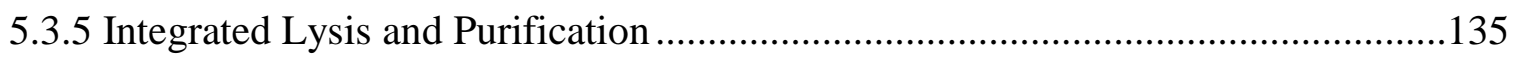

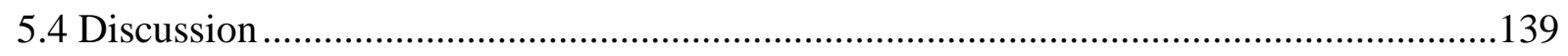

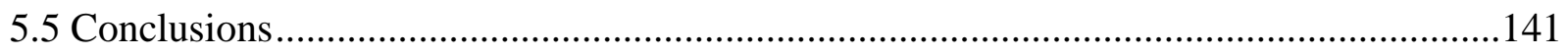

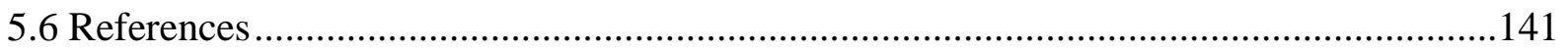

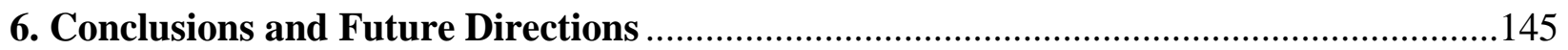

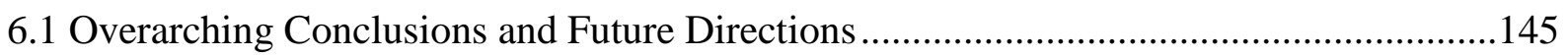

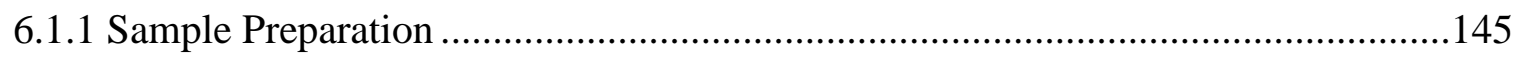

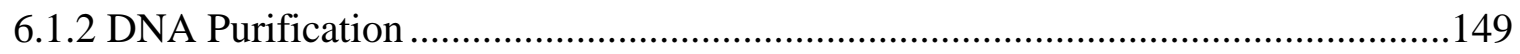

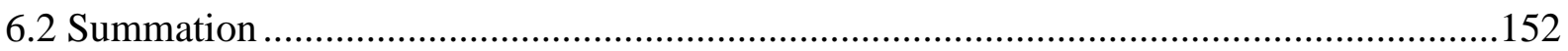

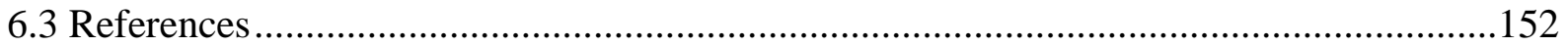




\section{Acknowledgements}

I would first like to thank Dr. Landers for his guidance and support over the past five years. You have taught me so much and always challenged me to be creative and achieve things that I never thought was possible. Also, the opportunities to participate in grant writing, human subject research upkeep, and patent writing were invaluable experiences that taught me skills useful in my future career. Over the years I have grown significantly as a scientist and for that I am truly grateful. I also would like to thank my mentors at the Defense Forensic Science center, Dr. Karen Olson and Dr. Roman Aranda. Your knowledge of forensics and experimental guidance throughout my summer internships were crucial to my success as a graduate student, and I cannot thank you all enough for your support.

I am very thankful for all of those who worked with me on projects over the years both in the Landers laboratory and through collaborations. A special thanks goes to Dr. Gavin Garner, Maximo Meija, and Daniel Mills who built and maintained a centrifugal system that allowed me to obtain a significant amount of data for this dissertation. I also am grateful for the advice and support from current and previous members within the Landers laboratory over the years. A special thanks goes to Dan Nelson for mentoring me through the early stages of my graduate career and Dr. Jingyi Yi for your collaboration on all of my projects. I had the pleasure of working with several undergraduates including Elisabeth Kamano and David Dent who both worked very hard. I'm very appreciative of all of the things you all helped me achieve and for the friendship we have. I especially want to thank Juliane Borba for all of her collaborative work on our project and for your friendship. Words cannot express how much I appreciate everything that you have done for me through the good and bad times. 
I am very appreciative of the support of my church, The Point, my community group, and all of my friends in Charlottesville. You all are like family and your encouragement helped me throughout graduate school. I would like to especially thank my "adopted parents", Lanny and Suzanne White for everything that you all have done throughout my time in graduate school. You accepted me as one of your own and I will always cherish those friendships. I would also like to thank my immediate family for their continuous support and love. You all always led me to look at God's truths and encouraged me to keep putting one foot in front of the other. For that reason, I dedicate this dissertation to you all. 


\section{Introduction}

\subsection{Overview of Forensic Genetic Analysis}

DNA analysis is among the most technologically advanced areas of forensic science due to several critical discoveries in the late $20^{\text {th }}$ century. In 1985, Sir Alexander Jeffreys found oligonucleotide sequences ${ }^{1}$, or 'minisatellites' ${ }^{2,3}$, in human genomic DNA which are repeated units (6-100 base pairs) throughout DNA and highly variable between individuals. Coupling multiple loci of these variable number of tandem repeats (VNTR) together could differentiate a unique pattern, specific to each individual, and was later called "DNA fingerprinting"4. To do this, radio-labeled VNTR probes hybridized to DNA (previously digested by restriction enzymes) and were exposed to X-ray film for visualization of the profile. This technique, also called restriction fragment length polymorphism (RFLP), affords a high degree of discriminating power but required substantial amounts of high molecular weight DNA and lacked the ability to delineate origins of mixed samples ${ }^{5}$. To simplify this process and decrease statistical errors of assigning fragments within loci, single-locus profiling (SLP) was used ${ }^{4}$.

The discovery of polymerase chain reaction (PCR) aided RFLP analysis with improved sensitivity, speed, and genotyping precision. Originally discovered in December 1985 by Dr. Kary Mullis, this method used two oligonucleotides to hybridize on either side of a targeted region of DNA $^{6}$. With careful temperature cycling in the presence of an optimized buffer, dNTPs, and a polymerase, an exponential increase of 220,000 was observed within 1 day from less than $1 \mu \mathrm{g}$ of DNA template. Due to the high quality and large quantity of DNA required for analyzing VNTRs, advancements were made towards using smaller 'microsatellites' instead. These microsatellites, or short tandem repeats (STRs), are repeating units of 2-6 base pairs and are found in noncoding regions of chromosomes within the genome. These STRs can easily be coupled to PCR technology 
for easier analysis of degraded or low template samples that is not possible with RFLP ${ }^{7}$. In addition, the decreased number of repeating units associated with STRs allow more loci to be analyzed simultaneously in a given sample; hence STRs may allow for increased discrimination

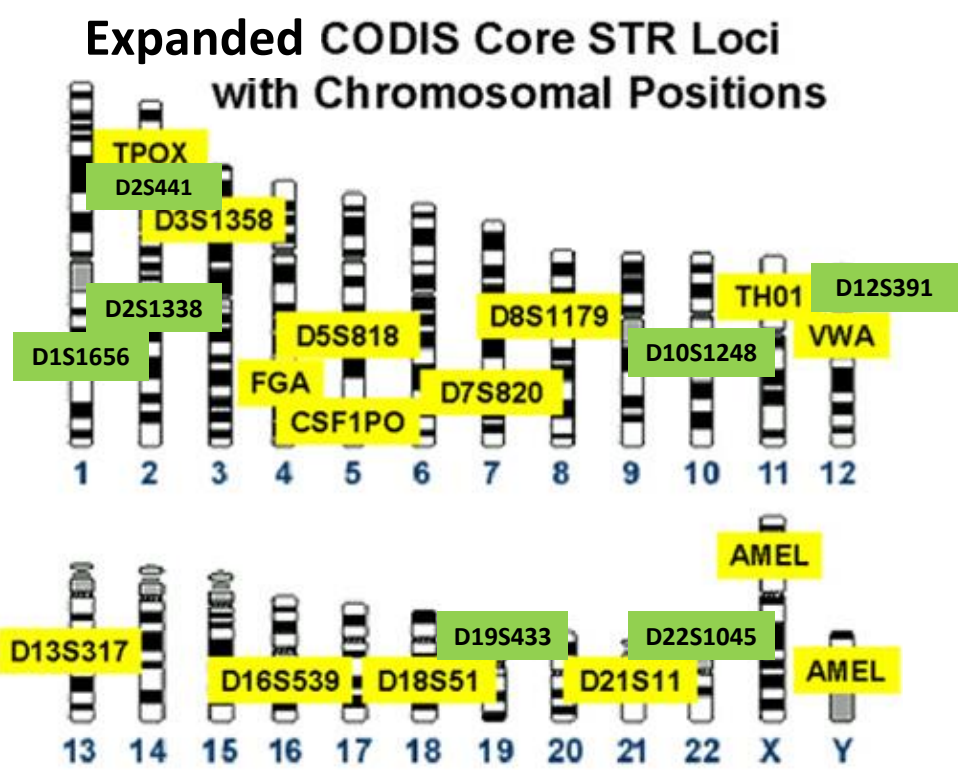

Figure 1: Chromosomal positions of 13 Core Loci (yellow) with expanded European Loci (green) selected by the FBI. Adapted from http://nitro.biosci.Arizona.edu/courses/EEB2082008/Lecuture08/Lecture08.html with expanded loci information from Thanakiatkrai et al ${ }^{8}$. among individuals.

Since 1997,13 of these STR

loci have been viewed by the

Federal Bureau Investigation (FBI) as the most informative PCR-based biological markers available to differentiate individuals ${ }^{9}$. The current STR kits allow for discrimination of $10^{14}$ which surpasses the world population of $10^{9}$ individuals. On January 1, 2017, these 13 core loci will be combined with 7 more to increase the international compatibility and improve the discriminating power between individuals ${ }^{10}$ (Fig. 1).

Several sample preparation and quantitation steps are required prior to obtaining an STR profile of the STR loci. The steps are outlined in Figure 2 and are broken down into the following: presumptive testing, DNA lysis and extraction, DNA quantitation, DNA amplification, and finally separation and detection. This process usually takes approximately 7-10 hours to complete depending on the sample of interest ${ }^{5}$. Attempts have been made to decrease the sample preparation 


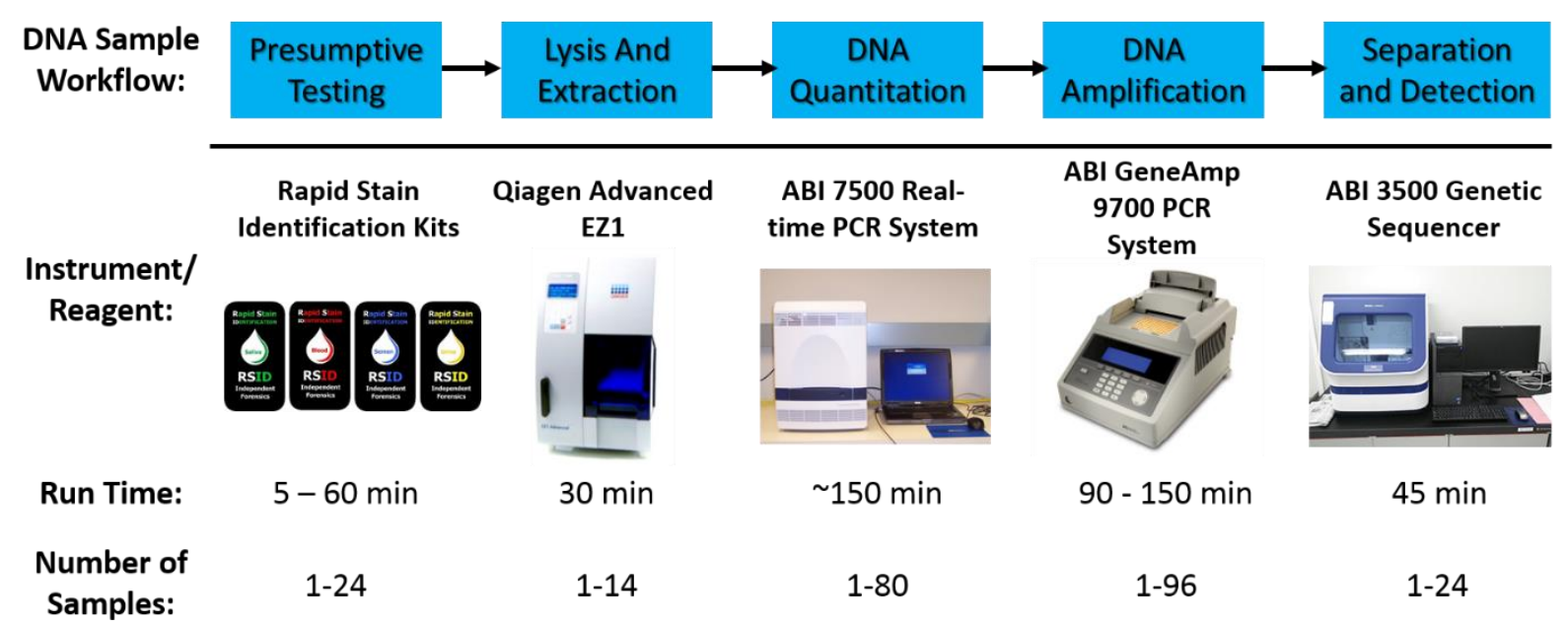

Figure 2: DNA analysis sample workflow with conventional instruments or reagents and associated run times ${ }^{12}$.

time with direct amplification of samples following lysis (bypassing purification and quantitation procedures), but this process is still being optimized with reference samples ${ }^{11}$.

\subsection{Presumptive Testing}

Evidence submitted to a crime lab is commonly screened for traces of DNA in an effort to save DNA analysis reagents and time. Most of these screening tests are presumptive and look for DNA in the presence of different body fluids including blood, saliva, and semen ${ }^{5}$. There currently are no adopted forensic lab methods for sweat, urine, vaginal secretions, or specifically for menstrual blood.

Presumptive testing via body fluid identification is also useful because it can provide critical contextual information that is not provided by a DNA genetic profile. Male DNA found on a female victim's clothing, for instance, may not be as significant as semen found on vaginal $\operatorname{swabs}^{13}$. Therefore, understanding the origin of the male DNA on the clothing and the method of DNA transfer is key for investigative purposes as it can significantly change the investigation in a 
criminal case. However, in testing these body fluids, it is important to aim for methods that are nondestructive and are easily integrated into the DNA sampling workflow towards obtaining a STR profile ${ }^{14}$.

\subsubsection{Overview of Sample Processing}

All pieces of evidence submitted to a forensic lab are inspected for the presence of body fluids. Stains, both visible and invisible to the naked eye are identified with the help of ultraviolet light or alternative light sources, then tested one at a time using presumptive colorimetric tests. Any positive results with presumptive tests are followed by confirmatory testing for the identification of a particular body fluid. Most of these testing methods are destructive to the sample, so it is imperative that an examiner be discerning in choosing which tests to try ${ }^{15,16}$. This is not always an easy task, as many of the invisible stains are not visually distinguishable from each other.

\subsubsection{Blood}

Blood is commonly found at crime scenes, and is frequently identified with the detection of hemoglobin via presumptive colorimetric identification, immunoassays, and alternative light sources (Fig. 3). The Kastle Meyer test with phenolphthalein is the most popular colorimetric presumptive blood test due to its high sensitivity and rapid transition to a bright pink color in the presence of blood ${ }^{17}$. Due to the high risk of false positives, a less sensitive yet more specific colorimetric assay using tetramethylbenzidine (TMB) is sometimes used in combination with

phenolphthalein. TMB blood tests, such as Hemastix ${ }^{\circledR} 18$ (Lynn Peavy Company, USA), will rapidly turn a sample test strip from orange to blue-green in the presence of blood. Even with 
A

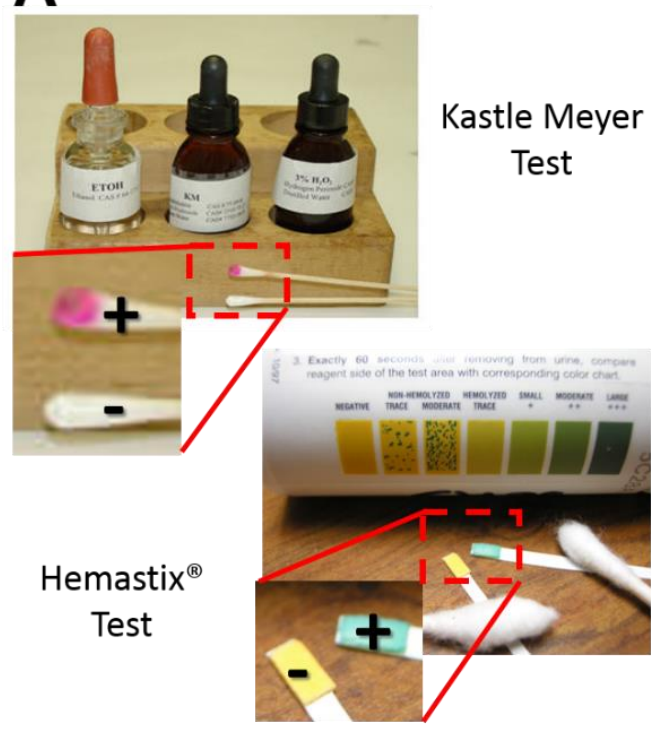

Leucomalachite-green-test with $10 \%$ ferrous sulphate: Before $\mathrm{H}_{2} \mathrm{O}_{2}$;
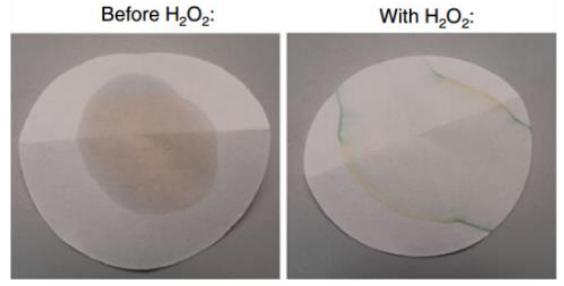

B
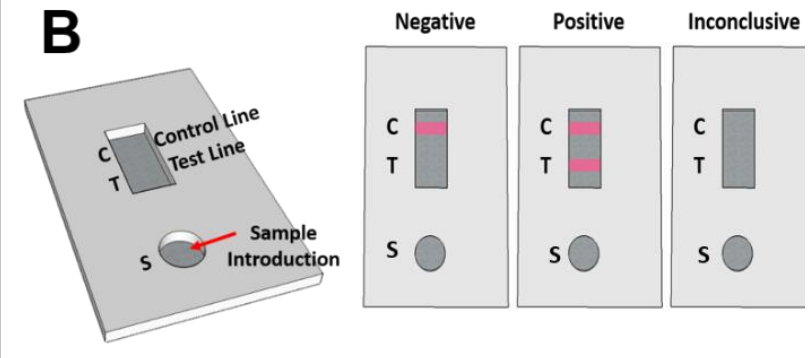

RSID ${ }^{\mathrm{TM}}$ Field Kit: $\quad$ Blood Volume analyzed $(\mu \mathrm{L}): 5.3 \quad 5.3$

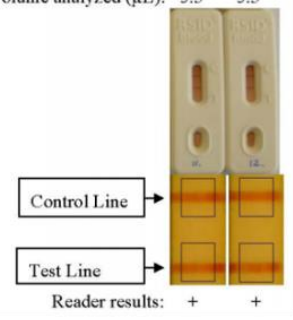

ABAcard® Hematrace

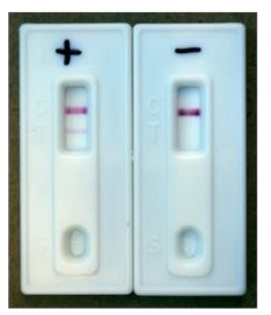

C

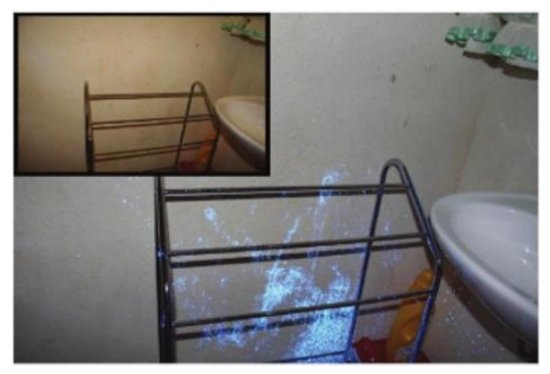

Figure 3: Presumptive and Confirmatory tests for Blood. (A) Presumptive colorimetric blood tests examples including the Kastle Meyer (top), Hemastix ${ }^{\circledR}$ (middle) and leucomalachite- green (bottom) tests ${ }^{5,22}$. (B) Immunological blood test examples ${ }^{23}$. Alternative light source blood detection via luminol spray ${ }^{24}$.

increased specificity, TMB tests are still subject to false positives ${ }^{19}$ and have been found to negatively affect downstream DNA extraction ${ }^{20}$. A third presumptive colorimetric test for blood is the leucomalachite green (LMG) test. It is not commonly used because it is a known carcinogen, interferes with downstream STR processing, and lacks specificity to human blood ${ }^{18,21}$.

Although colorimetric testing is useful for visual stains, it is not practical to use these tests on items where blood cannot be seen. In these cases, analysts rely on alternative light sources for the luminol test ${ }^{16,25}$. This reaction is catalyzed by iron(II) in blood which oxidizes luminol to produce 3 -aminophthalate ${ }^{15}$. This reaction emits a blue light as it relaxes from its excited state and 
can be observed with the naked eye $\mathrm{e}^{13,26}$. Although luminol can pinpoint the locations of possible blood stains and is non-toxic, it may have detrimental effects on downstream STR processing depending on the formulation ${ }^{27-29}$.

Immunoassays are popular confirmatory tests for the presence of blood and are used to validate presumptive test results from luminol and colorimetric testing. Immunoassay tests target antigens within blood to provide increased specificity and improved limit of detection down to $0.07 \mu \mathrm{g} / \mathrm{mL}^{30}$. Some of these tests include ABAcard ${ }^{\circledR}$ HemaTrace $^{\circledR}$ (Abacus Diagnoistics ${ }^{\circledR}$, USA), SERETEC ${ }^{\circledR}$ HemDirect (SERATEC Gesellschaft für Biotechnologie mbH, Germany), and Rapid Stain Identification (RSID ${ }^{\mathrm{TM}}$; Independent Forensics, USA) of blood ${ }^{5,15,31}$. Most of these tests target hemoglobin and are therefore not human specific ${ }^{5,30}$. For this reason, the RSID ${ }^{\mathrm{TM}}$ immunoassay targeting glycophorin A is preferred due to the lack of cross-reactivity ${ }^{32}$. Other confirmatory yet impractical methods include spectroscopic methods, chromatography, and microscope identification of red and white blood cells ${ }^{14,15}$.

\subsubsection{Semen}

The identification of seminal stains are critical in cases involving sexual assault, which encompass approximately $2 / 3$ of cases pursued with DNA evidence ${ }^{5}$ Three methods are used for the identification of semen: colorimetric testing via acid phosphatase (AP), prostate specific antigen (PSA), or microscopic identification of sperm cells (Fig. 4). AP is the most common colorimetric test for semen because it is highly expressed in semen at concentrations between 500 to 1000 times higher than in other body fluids ${ }^{25,33}$. If semen is present, a purple color will rapidly appear following contact with a napthyl phosphaste and diazo blue dye solution. Unfortunately, AP is still detectable in other body fluids including vaginal secretions so this test is not specific to 
semen $^{19}$. This colorimetric AP test can also result in false negatives, so overall it is considered to be highly presumptive.

PSA is highly expressed in the prostate gland with concentrations from $300 \mathrm{ng} / \mathrm{mL}$ up to $4200 \mathrm{ng} / \mathrm{mL}$ and is even detectable in oligospermic (low sperm) and azospermatic (no sperm cells) samples $^{5,34}$. Several immunological tests for the detection of PSA are available including SERATEC $^{\circledR}$ PSA (SERATEC Gesellschaft für Biotechnologie mbH, Germany) and OneStep ABAcard $^{\circledR}$ PSA $^{35}$ (Abacus Diagnostics, USA). SERATEC ${ }^{\circledR}$ is more sensitive than the ABAcard ${ }^{\circledR}$, however, it is susceptible to false positives with condom lubricants. Although PSA provides the sensitivity needed to detect semen, PSA can also be found in other body fluids including blood, vaginal fluid, and urine ${ }^{36,37}$, and PSA tests are susceptible to false positives when condom lubricants are present and on anal swabs ${ }^{38,39}$.

RSID sperm tests and microscopy are used as confirmatory tests following AP or PSA testing. Semenogelin, a prominent protein in semen, is used to detect semen in RSID ${ }^{\text {TM}_{-}}$Semen immulogical tests (Independent Forensics, USA). An independent study found that RSID ${ }^{\mathrm{TM}}$ Semen tests targeting semenogelin are less sensitive than SERATEC ${ }^{\circledR}$ PSA but they eliminated all false negatives ${ }^{40}$. Several stains can assist in microscopic visualization of sperm cells and include the Christmas Tree stain, 'spermpaint', and the PERM HY-LITER PLUS stain. The Christmas Tree stain is a combination of two stains used to identify sperm cells. For this stain, the sperm head will be stained by Nuclear Fast Red stain while the picroindigocarmine stains the sperm tails (if present) in green and blue. ${ }^{34}$ Unfortunately, heavy training is required in identifying sperm cells when the sperm tails are not present in a sample. Even with the positive identification of sperm cells, there is no method for defining only spermatozoa of human origin. Stains that integrate monoclonal antibodies for sperm cells have increased sensitivity and specificity over the 


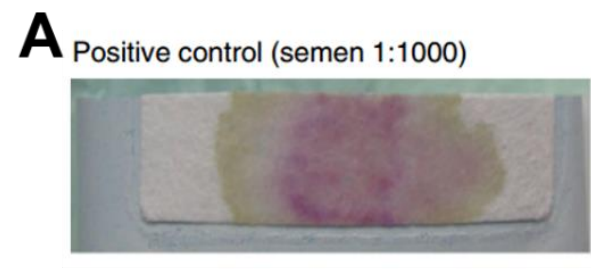

Negative control (water)

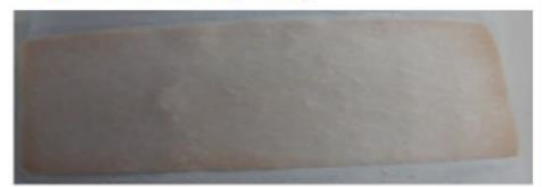

C

Semen volume $(\mathrm{nL}): 0 \quad \begin{array}{llllll}0.5 & 5 & 10 & 50 & 0\end{array}$

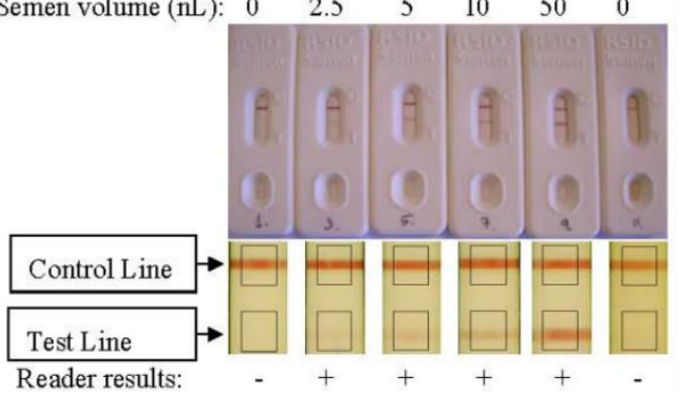

B

\begin{tabular}{|c|c|c|c|c|c|c|}
\hline PSA & Seratec $^{1}$ & Seratec $^{2}$ & Seratec $^{3}$ & $\mathbf{A B A}^{4}$ & $\mathbf{A B A}^{3}$ & $\mathbf{A B A}^{3}$ \\
\hline $\mathbf{5 0 0}$ & + & + & + & + & + & + \\
\hline $\mathbf{1 0 0}$ & + & + & + & + & + & + \\
\hline $\mathbf{5 0}$ & + & + & + & + & Weak + & + \\
\hline $\mathbf{2 5}$ & + & + & + & + & Spot + & + \\
\hline 12.5 & + & + & + & + & Faint + & + \\
\hline 6.25 & + & + & + & Weak + & - & - \\
\hline $\mathbf{2 . 1 3}$ & + & + & + & - & - & - \\
\hline 1.56 & + & + & + & - & - & - \\
\hline $\mathbf{0 . 7 8}$ & - & + & + & - & - & - \\
\hline
\end{tabular}

D

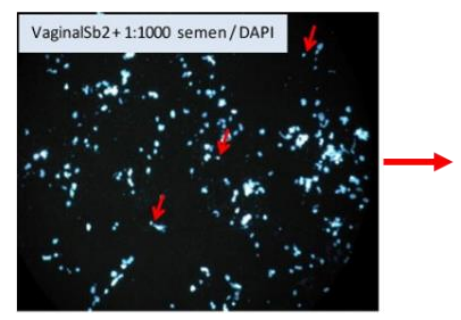

Figure 4: Presumptive and Confirmatory tests for Semen. (A) Presumptive colorimetric acid phosphatase test example ${ }^{22}$. (B) Comparison chart between two presumptive PSA immunological tests for semen ${ }^{43}$. (C) Sensitivity study for confirmatory RSID ${ }^{\mathrm{TM}}$ test $^{23}$. (D) Microscopic image of DAPI stained epithelial cells with intermixed stained sperm cells via Sperm Hy-Liter ${ }^{\mathrm{TM}}$. With a DAPI filter, only sperm cells are visible ${ }^{42}$.

Christmas Tree stain, and can identify small amounts of sperm cells in a given sample. A 'spermpaint' method was developed by John Herr at The University of Virginia, and uses two monoclonal antibodies to selectively label the head and tail of sperm cells ${ }^{41}$. A more sensitive SPERM HY-LITER PLUS stain method (Independent Forensics, USA) is preferred and uses human-specific monoclonal antibodies to detect even a single human sperm cell, and has since been validated as a viable microscopy method that does not disrupt downstream STR processing ${ }^{42}$. 


\subsubsection{Saliva}

Saliva stains can be found on miscellaneous items at a crime scene including envelopes, cigarette butts, and drinking vessels ${ }^{44}$. Saliva stains, initially invisible to the eye, can be located using an alternative light source. Once located, analysts

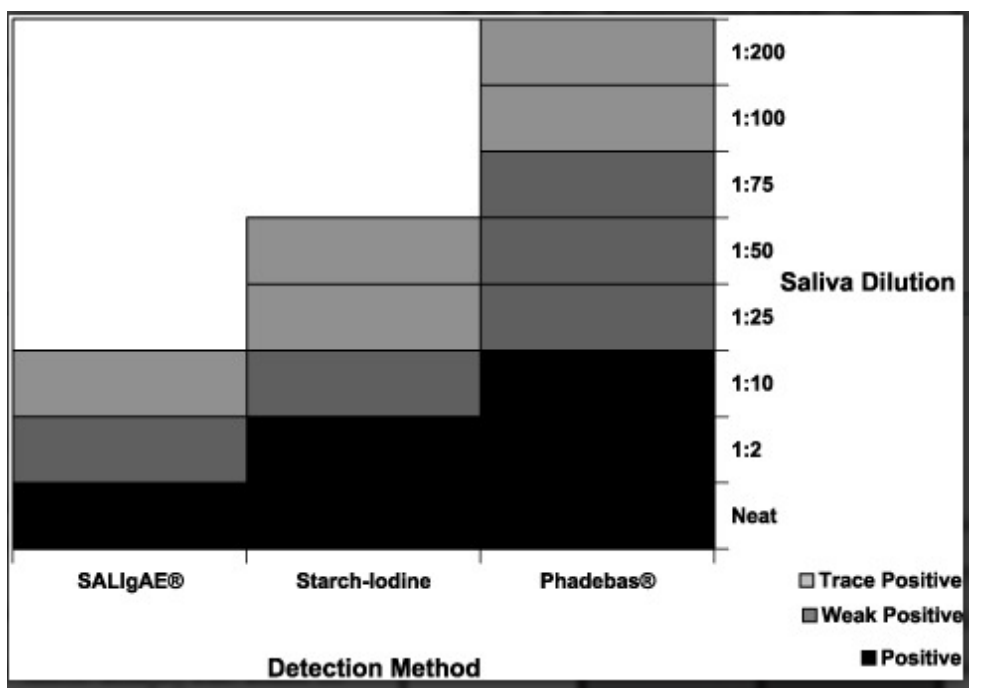
commonly use the starch iodine test or a Phadebas ${ }^{\circledR}$ test (Magel Life tests ${ }^{45}$.

Figure 5: Sensitivity comparison of presumptive saliva Sciences, Sweden) which both look for the presence of $\alpha$-amylase. These presumptive tests provide inexpensive and sensitive detection, but lack fluid specificity, as $\alpha$-amylase can be found in breast milk, sweat, semen, vaginal fluid, etc ${ }^{14,25}$. A newly developed saliva test, SALIgAE ${ }^{\circledR}$ (Abascus Diagnostics, USA), is a proprietary mixture that was found to have equal or improved specificity to the starch-iodine and Phadebas ${ }^{\circledR} \operatorname{tests}^{45}$ and can reportedly detect trace saliva samples $^{46}$. However in an independent study, the SaLIgAE test was found to be less sensitive than other presumptive tests (Fig. 5) $)^{45}$. The RSID ${ }^{\mathrm{TM}}$ saliva test can be used as a confirmatory saliva test for more sensitive and specific detection of human $\alpha$-amylase ${ }^{46,47}$, but still provides false positives from citrus juices ${ }^{48}$.

\subsubsection{Emerging Techniques}

Efforts have been made in developing nondestructive body fluid identification techniques with increased specificity for blood, sperm, and saliva while expanding to other body fluids such 
as vaginal fluid, sweat, urine, and menstrual blood. Several characteristics of body fluid identification methods are kept in mind during the development of new detection methods: (i) simplicity and nondestructive to sample; (ii) high fluid specificity; (iii) low cost; (iv) easy integration into current DNA workflow, and (v) minimal training ${ }^{14}$.

The development of specific tests for body fluids beyond blood, sperm, and saliva is not trivial as many of the identified markers are not unique to one body fluid. Positive identification of urea, for instance, is a common indicator for urine, however, it is also highly expressed in sweat ${ }^{5}$. Despite these limitations, several methods utilizing RNA markers, protein markers, bacteria, and DNA methylation patterns have been explored for body fluid identification with increased specificity.

\subsubsection{RNA-based Methods}

There are significant differences in gene expression of the cells likely to be present in the different body fluids. Investigating the RNA that controls the expression of specific genes could provide clues for how to differentiate body fluids with increased accuracy. RNA, previously thought to be unstable over time, has now been widely accepted as a relatively stable indicator for body fluid identification ${ }^{49,50}$. Comprehensive studies on RNA stability revealed that RNA can be detected in dried samples up to 180 days $^{51}$ and are even detectable in some samples after 547 days $^{52}$. Various forms of RNA, primarily messenger RNA (mRNA) and micro RNA (miRNA), have been useful for identifying body fluids with high specificity using tissue-specific expression markers $^{25}$.

One of the most significant advantages in using mRNA for body fluid identification is the simple integration into the current DNA workflow via co-purification of RNA with DNA ${ }^{53-55}$. 
Table 1 lists several mRNA markers that have been identified by many groups ${ }^{49,56-58}$ as possible fluid-specific markers for body fluids due to increased expression levels in a particular body fluid. The detection sensitivity of these mRNA markers are comparable to presumptive tests and have potential for detection of body fluids with increased accuracy ${ }^{59}$. Thus far, four primary methods

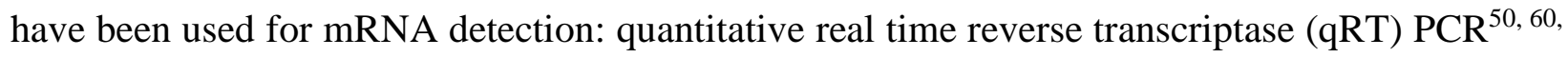
61, Nanostring ${ }^{\circledR}$ hybridization technology $5^{56,}{ }^{58}$, mass spectrometry $(\mathrm{MS})^{62}$, and turbidity/fluorescence analysis ${ }^{3}$. Of these methods, PCR and Nanostring ${ }^{\circledR}$ technology have demonstrated the potential for multiplexing the detection of body fluid types to include blood, saliva, semen, and vaginal fluid detection into one assay. A matrix of 4-5 mRNA markers per fluid were used for more accurate fluid identification, however, some of these mRNA markers (especially vaginal fluid and menstrual blood) are also expressed in other body fluids and are not easily differentiated. In such cases, statistical algorithms are used to determine the probability of the presence of particular body fluids ${ }^{44,58}$. To minimize the need for statistical algorithms, only the most fluid-specific blood marker was chosen for a highly specific isothermal amplification method with fluorescence detection. This simplified process was reportedly more sensitive than traditionally used confirmatory tests for blood and could be useful for trace analysis. To date, this method has only been demonstrated with blood detection ${ }^{3}$, but could be applied to the other body fluids.

Due to the degradation effects of mRNA in the presence of heat and humidity, researchers have looked towards using miRNA markers for body fluid identification. Unlike mRNA, miRNA is noncoding RNA that is responsible for post-transcriptional gene expression ${ }^{63}$. Once matured, these markers extend between 19-25 nucleotide bases long and have long lifetime stabilities due to decreased susceptibility to environmental factors ${ }^{64}$. Several miRNA markers have been 
Table 1: mRNA Proposed Markers ${ }^{3,5,50,56,58}$

\begin{tabular}{|c|c|c|c|c|c|c|}
\hline Blood & Saliva & Semen & Vaginal Fluid & Menstrual blood & Skin & Housekeeping \\
\hline HBB & HTN3 & SEMG1 & MUC4 & MMP7 & CCL27 & 18S RNA \\
ALAS2 & KRT16A & TGM4 & HBD1 & MMP11 & 1L1F7 & B2M \\
SPTB & STATH & PSA & SERPINB3 & LEFTY2 & KRT9 & GAPDH \\
PBGD & FDCSP & PRM1 & CYP2A7 & MMP10 & LCE1C & HMBS-A \\
HBA1 & MUC7 & PRM2 & CYP2B7P1 & & LCE2D & HMBS-B \\
PPBP & KLK4 & KLK3 & DKK4 & & & -actin \\
NKG7 & HTN1 & MSMB & FUT6 & & & COX1 \\
CCL5 & SRM3B & NKX3-1 & IL19 & & & HPRT1 \\
NRGN & & & MYOZ1 & & & PGK1 \\
GZMH & & & NOXO1 & & & PP1H \\
PRF1 & & & & & & S15 \\
GlycoA & & & & & & TFEA1 \\
PF4 & & & & & & UBC \\
ANK1 & & & & & & UBE2D2 \\
\hline
\end{tabular}

proposed for unique identification of body fluids including blood, saliva, and vaginal fluid ${ }^{64-69}$. Unfortunately, very few of the markers identified have been verified by multiple studies due to controversial results ${ }^{65}$. For instance, nine markers were identified for peripheral blood, but of these markers, only two, miR-451 and miR-16 were identified in multiple studies ${ }^{14}$. Unstable gene expression of miRNA is thought to blame for the mixed results ${ }^{14}$. More research must be completed before miRNA markers can be employed for body fluid sample identification in forensic analysis.

\subsubsection{DNA Methylation Patterns}

The human body exhibits a unique epigenome that may be useful to differentiate body fluids for identification. DNA methylation occurs at the $5^{\prime}$ position of cytosine in cystosine phosphate - guanidine $(\mathrm{CpG})$ regions, and is responsible for maintaining cell differentiation through the regulation of gene expression ${ }^{70}$. Different types of cells have a unique methylation profile which can indicate 'tissue specific differentially methylated groups' (tDMRs) that are both stable and specific ${ }^{70,71}$. Several tDMRs have been proposed for body fluid identification following 
the analysis of cellular methylation patterns ${ }^{72-75}$. The primary methods for testing these tDMRs are bisulfite sequencing ${ }^{72,75,76}$ and restriction enzyme-treated $\mathrm{qPCR}^{77,78}$. Bisulfite treatment converts all unmethylated cytosines to uracil in a DNA sequence, which is then amplified to yield a mutated sequence. Restriction enzymes, on the other hand, cleave all unmethylated $\mathrm{CpG}$ locations which can prevent amplification of target sequences if the target DNA sequence is digested. As shown in Figure 6, both of these methods are useful for the identification of body fluids, such as semen. Nucleix DSI Semen ${ }^{\mathrm{TM}}$ (Nucleix, Israel) is a commercial DNA methylation technique that reliably detects semen when not mixed with other fluids without yielding false positives $^{79}$. Although reported tDMR markers are detectable with great sensitivity, more studies have shown that tDMRs, once thought to be fluid-specific, may show cross reactivity in male donors. Furthermore, studies have found differences in methylation patterns between individuals that may affect methylation analysis along with issues such as degradation of a sample, inhibition, and low template samples ${ }^{14}$. Overall, DNA methylation methods are promising for body fluid identification, but require further advancements with genetic marker mapping for more reproducible detection of methylation patterns ${ }^{14,75}$.

\subsubsection{Microbial Profiling}

The difficulty in identifying body fluids such as vaginal fluid and saliva due to the lack of fluid specificity via mRNA and methylation methods has led researchers to explore the human microbiome. Differences in the natural flora throughout the body can possibly be exploited for new methods of body fluid identification. Amplification of hypervariable regions of $16 \mathrm{~S}$ ribosomes in species-specific bacteria could allow specific identification of body fluids that could 

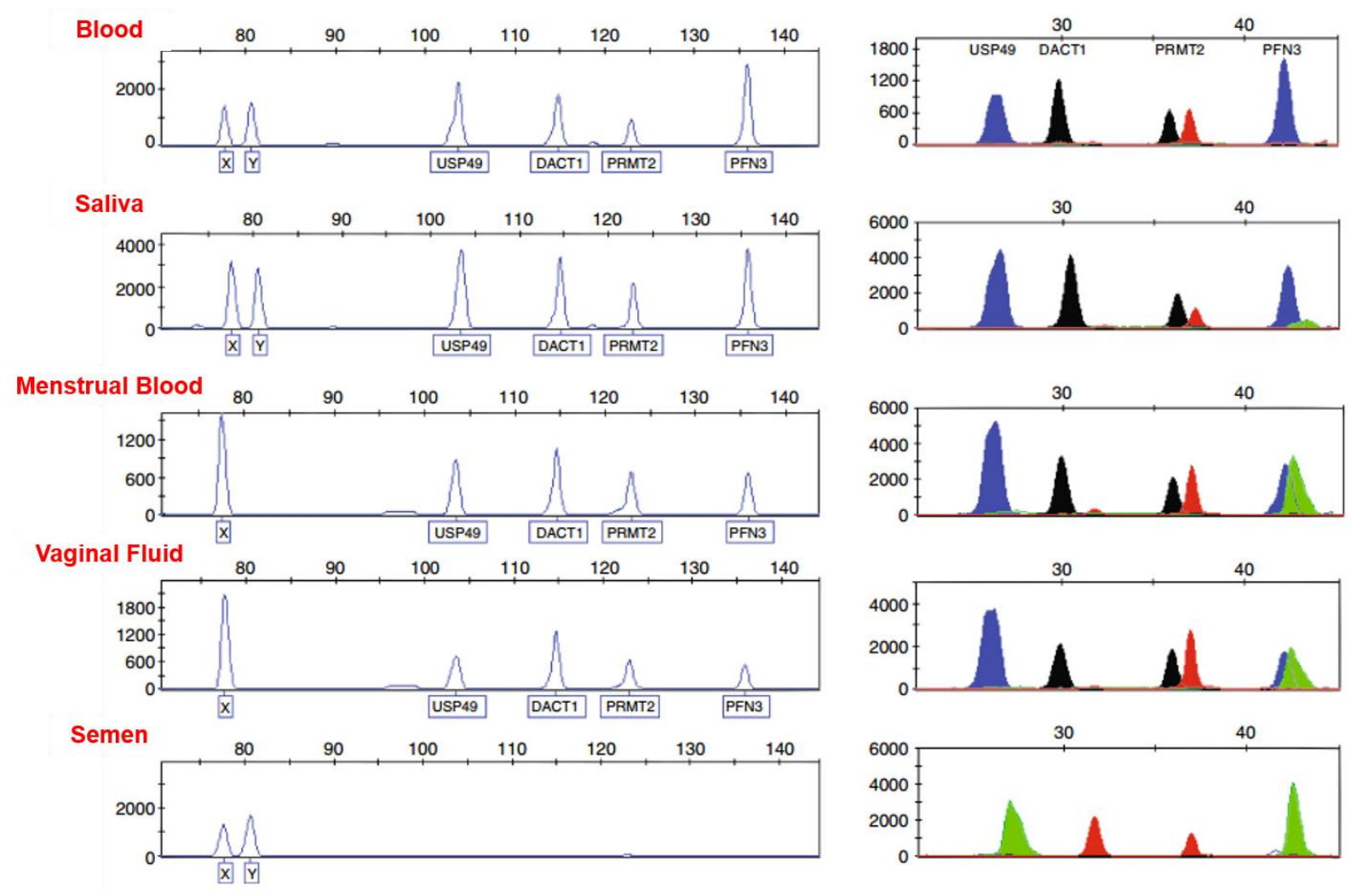

Figure 6: Identification of semen based on the amplification of DACTI, USP49, PRMT2, and PFN3 tDMRs following methylation sensitive restriction enzyme (MSRE) PCR (left) and bisulfite sequencing (right) ${ }^{76}$. Only in semen are tDMR sequences unmethylated at $\mathrm{CpG}$ sites at all of these loci. Following MSRE PCR, amplicons for all tDMRs are found with exception of semen. In bisulfite sequencing, the methylation sites result in base mutations that can be identified through fluorescent detection. The green and red peaks signify base mutations and together, the tDMRs can uniquely discriminate semen from all other examined body fluids.

not easily be identified otherwise ${ }^{75}$. Microbial methods, however, must account for inter person variability, as age and health can largely effect the concentrations of detectable bacteria.

Bacterial markers have largely been studied for saliva, vaginal fluid, and feces. Several signatures for vaginal fluid utilize Lactobacillus crispatus, Lactobacillus gasseri, Lactobacillus iners, and Lactobacillus jensenii ${ }^{80}$. The concentrations of these lactobacilli bacteria are known to vary throughout the menstral cycle but are always present. A detectable amount of Garderella vaginalis is also found in women that are experiencing bacterial vaginosis ${ }^{81}$. 
Bacterial markers identified for saliva are Stretococcus salivarius and Stretococcus mutans which have successfully been used for the detection of saliva via isothermal amplification $^{82}$, or in combination with DNA methylation methods ${ }^{81}($ Fig. 7). However, small detectable amounts of these saliva markers have also been found in feces ${ }^{83}$. Despite this, these bacterial signatures were reported for successful differentiation of vaginal fluid, saliva, and feces via $\mathrm{qPCR}^{83}$. Saliva, in this case, was indirectly 'confirmed' by verifying the absence of all bacteria commonly sourced to fecal matter of any species (i.e. Enterococcus genus).

Since bacterial species are naturally present on surfaces and other parts of the body including hands and the groin ${ }^{14}$, detection of bacterial markers may not be a reliable method for validating the presence of particular body fluids. One study employed multiplexed PCR methods for simultaneous identification of blood, vaginal fluid, saliva, semen, and fecal matter, utilizing microbial signatures in combination with methylation markers ${ }^{83,84}$. Although microbial profiling in its current stage cannot identify body fluids with confidence, it can provide useful additional markers for identification of difficult body fluids, such as vaginal fluid.

\subsubsection{Protein Markers}

The identification of protein markers, originally intended for cancer and other diseases, have shown to be useful in body fluid identification ${ }^{85}$. Using proteins instead of RNA increases the diversity of targets available due to post translational modifications within each body fluid ${ }^{86}$. Proteins are also incredibly stable,

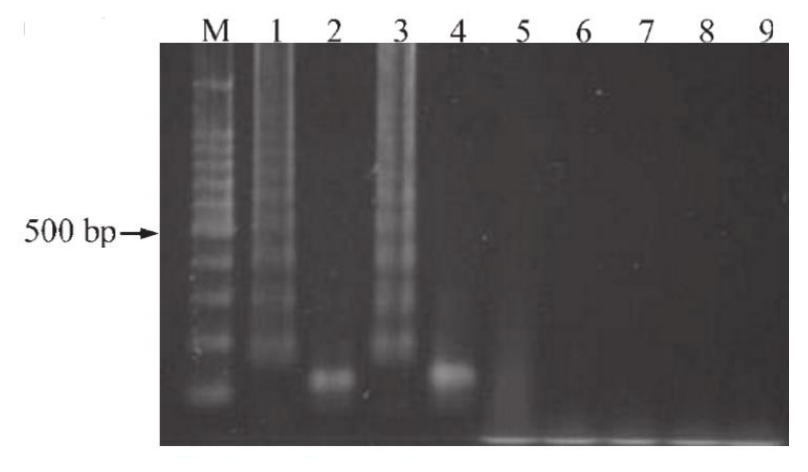

Figure 7: An electrophoretic gel of: purified S. salivarius (lane 1); Cel II digested amplified product of S. Salivarius (lane 2 and 4); amplified saliva sample (lane 3); and nonspecific controls with semen (lane 5), urine (lane 6), vaginal fluid (lane 7), and skin (lane 8) samples ${ }^{82}$. 
and are routinely used for bioanalysis of ancient biological material, which is ideal for body fluid identification $^{87}$.

Chromatography was one of the first methods for both discovery of new proteins and for verifying and validating markers ${ }^{88}$. Chromatographic methods, such as high performance liquid chromatography (HPLC) is combined with spectrometry analysis to separate digested and whole proteins and analyze the contents in a given sample. Using HPLC coupled with a matrix-assisted laser desorption ionization time of flight (HPLC-MALDI TOF) detector, the proteome of blood, semen, and saliva were compared to known profiles from various other human body fluids (including clinically relevant fluids such as cerebralspinal fluid, plasma, serum, etc) for unique protein markers ${ }^{89}$. From 100 samples, four proteins were found to be unique to blood (HBB, HBA1/HBA2, SLC4A1, and SPTA1), five proteins unique to saliva (AMY-1, SMR3B, C6orf58, CYS2, and HTN1), and eight proteins were unique to semen (SEMG1, SEMG2, ACPP, KLK3, MUC6, MSMB isoform PSP94, PAEP, and CRISP1) ${ }^{89}$. Protein fingerprints of each body fluid based on these markers were consistent with previous studies and allowed for reliable identification of each body fluid. In mixtures, however, only the most abundant proteins were used to identify each body fluid, as some of the less concentrated proteins are not observed in a diluted mixture. To account for interpersonal variability, fluids from a small population of donors were analyzed and those proteins found in all fluid types were used for protein fingerprints ${ }^{86}$.

Van Steedam et al. expanded the body fluid analysis to include menstrual blood, vaginal fluid, nasal secretions, urine, and feces ${ }^{90}$. A 'decision tree' method was used to identify the 9 body fluids using fluid biomarkers based on the abundance of the protein and fluid specificity. An overnight digestion and reverse-phase separation allowed for the detection of biomarkers with 10fold improved sensitivity over the presumptive tests. Mixtures were also easily identified via the 
'decision tree', however, the identification of menstrual blood first required positive identification of venous blood. If a mixed sample contained both venous and menstrual blood, it would not be possible to differentiate them.

The proteome method for body fluid analysis can be improved by eliminating the reverse phase column and analyzing both hydrophilic and hydrophobic peptides via direct body fluid analysis ${ }^{86}$. To do this, collected body fluids were digested and directly analyzed by a quadrupole TOF (Q TOF) mass spectrometer. Almost 700 proteins were found in these samples and more than $80 \%$ of candidate biomarkers for body fluids could be found in these unfractionated samples (Table 2). Using the proposed biomarkers, trace quantities of human semen, urine, vaginal fluid, venous blood, saliva, and menstrual blood were successfully detected following direct body fluid analysis $(n=12)$. Although specific biomarkers were identified, this method is limited by a laborious filtration step to eliminate high abundance, non-specific proteins that could hinder the ability to detect less abundant but more specific proteins. Also, due to a small population analysis $(\mathrm{n}=12)$, there is a possibility that biomarkers could be found in trace levels in nontarget fluids which could lead to inaccurate fluid identification.

Although the use of proteins for body fluid identification is in its infancy, there is significant potential for discovering markers with increased specificity due to the large diversity of potential protein targets. Proteins are also robust in harsh environmental conditions and are known to have long-term stability. Further research will facilitate the development of a multiplexed approach to body fluid identification which will be beneficial for confirmatory analysis of fresh samples, or older samples that could not be otherwise analyzed due to degradation. 


\subsection{Conventional Lysis and DNA Purification}

Once body fluid identification methods indicate the presence of DNA in the form of a body fluid such as blood or saliva sample processing moves forward to DNA purification. This involves releasing the DNA from the cell followed by separation of the DNA from all other cellular material using a method that is compatible with downstream processing. This is necessary as cellular components, such as hemoglobin, may inhibit subsequent PCR, which is required to obtain a STR profile.

Early purification of DNA from forensic samples relied on a chloroform-phenol extraction which exploits differences in cell component solubility between aqueous and organic phases. The phenol/chloroform mixture denatures proteins and partitions them into an organic phase while

Table 2: Protein Body Fluid Identification Markers Found in Unfractionated Samples ${ }^{86}$

\begin{tabular}{|c|c|c|c|}
\hline Body fluid & Protein & Accession & Unfractionated \\
\hline \multirow[t]{7}{*}{ Seminal Fluid } & Epididymal secretory protein E1 & P61916 & $\sqrt{ }$ \\
\hline & Glycodelin-S & P09466 & $\sqrt{ }$ \\
\hline & Semenogelin-1 & P04279 & $\sqrt{ }$ \\
\hline & Semenogelin-2 & 002383 & $\sqrt{ }$ \\
\hline & Prolactin-inducible protein & P12273 & \\
\hline & Prostate-specific antigen & P07288 & $\sqrt{ }$ \\
\hline & Prostatic Acid Phosphatase & P15309 & $\sqrt{ }$ \\
\hline \multirow[t]{6}{*}{ Saliva } & Cystatin_D & P28325 & $\sqrt{ }$ \\
\hline & Cystatin_SA & P09228 & $\sqrt{ }$ \\
\hline & Histatin-1 & $\mathrm{P} 15515$ & $\sqrt{ }$ \\
\hline & Salivary acidic proline-rich phosphoprotein $1 / 2$ & P02810 & \\
\hline & Statherin & P02808 & $\sqrt{ }$ \\
\hline & Submaxillary gland androgen-regulated protein $3 B$ & P02814 & $\sqrt{ }$ \\
\hline \multirow[t]{9}{*}{ Vaginal Fluid } & Cornulin & Q9UBG3 & $\sqrt{ }$ \\
\hline & IgGFc-binding protein & Q9Y6R7 & $\sqrt{ }$ \\
\hline & Involucrin & P07476 & $\sqrt{ }$ \\
\hline & Ly6/PLAUR domain-containing protein 3 & 095274 & $\sqrt{ }$ \\
\hline & Matrix metalloproteinase- 9 & P14780 & $\sqrt{ }$ \\
\hline & Neutrophil gelatinase-associated lipocalin & P80188 & $\sqrt{ }$ \\
\hline & Periplakin & 060437 & \\
\hline & Suprabasin & Q6UWP8 & $\sqrt{ }$ \\
\hline & Vimentin & P08670 & \\
\hline \multirow[t]{2}{*}{ Urine } & Osteopontin & P10451 & $\sqrt{ }$ \\
\hline & Uromodulin & P07911 & $\sqrt{ }$ \\
\hline Menstrual Fluid & Calpastatin & P20810 & \\
\hline \multirow[t]{4}{*}{ Peripheral Blood } & Alpha-1-antitrypsin & P01009 & $\sqrt{ }$ \\
\hline & Complement C3 & P01024 & $\sqrt{ }$ \\
\hline & Hemoglobin subunit beta & P68871 & $\sqrt{ }$ \\
\hline & Hemopexin & P02790 & $\sqrt{ }$ \\
\hline
\end{tabular}


leaving DNA in the aqueous phase. Although this method is successful, providing high purity, intact DNA from even difficult forensic samples, it is laborious and requires the use of hazardous reagents ${ }^{91}$.

The discovery of DNA binding to silica in $1979^{92}$ catalyzed the development of solid phase extraction (SPE) technology which is currently the most commonly used extraction method. To purify samples, following cell lysis, DNA is bound to silica particles in a high concentration chaotropic salt, such as guanidine hydrochloride $(\mathrm{GuHCl})$. An alcohol solution, such as $70 \%$ isopropanol, is used as a wash step to flush away contaminants and weak binding species. The DNA is then released from the beads in water or a low salt buffer, such as Tris-EDTA (TE) buffer. As shown in Figure 8, two primary forms of solid-phase extraction (SPE) exist: a packed silica phase or loose magnetic particles. In packed phases, solutions are passed through a static phase centrifugally or via syringe pumps or vacuum to purify DNA. Several commercial kits exist for SPE purification of DNA, produced by companies such as QiaAmp® (Qiagen), Wizard® Plus (Promega), PureLink ${ }^{\circledR}$ Pro (Thermofisher scientific), and others.

An alternative form of solid phase extraction is by utilizing loose silica particles with an iron magnetic core. These particles are free to move through a solution to capture DNA reducing the diffusion distance, then can be collected via an external magnet. Several biorobotic instruments, such as the EZ1® Advanced (Qiagen) and Maxwell@ systems (Promega) allow for full automation of the extraction process and are routinely used in forensic labs for casework processing.

Numerous other methods have been used for DNA purification and include chelex, FTA ${ }^{\mathrm{TM}}$, Chargeswitch ${ }^{\circledR}$, and enzyme liberation. Chelex extractions exploit an ion-exchange resin that bind polyvalent metals to deactivate nucleases. To purify samples, a $5 \% \mathrm{w} / \mathrm{v}$ of chelating resin is 


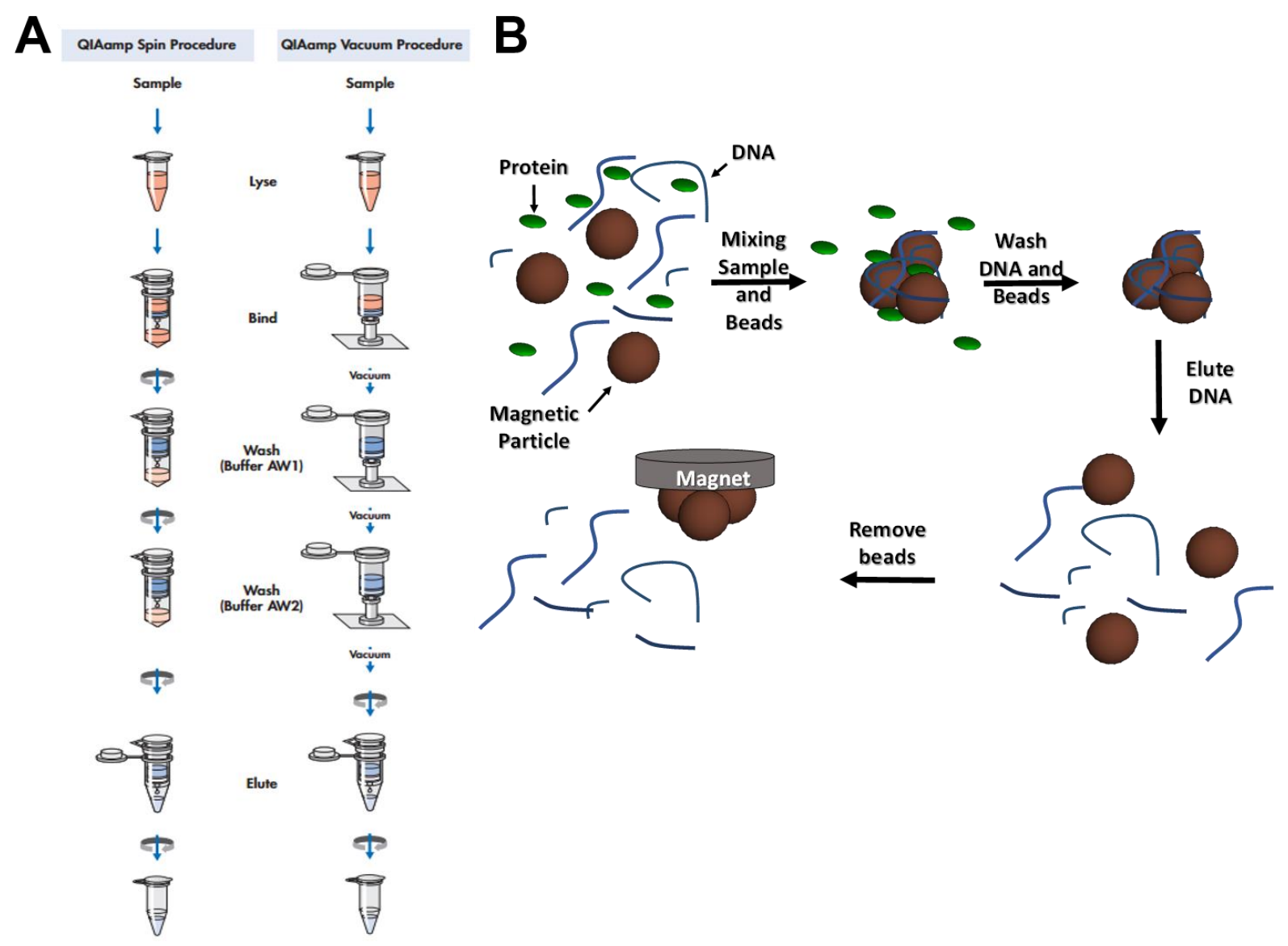

Figure 8: Solid Phase Extraction Methods. (A) Packed phase centrifugation or vacuum example procedure $^{93}$. (B) Schematic of dynamic solid phase extraction (dSPE) ${ }^{94}$.

placed in a sample and boiled for several minutes to release the DNA from cells. A short centrifugation step then pulls all cellular material to the bottom of a tube, leaving purified DNA in the supernatant. This method is popular due to minimized contamination risks, but can introduce PCR inhibition if too much sample is added ${ }^{5}$. Similar to Chelex is an extraction by FTA ${ }^{\mathrm{TM}}$ paper, a cellulose material that contains proprietary chemicals to stabilize nucleic acids for up to 1 year ${ }^{95}$. Upon contact with the paper, cells from blood or saliva samples lysed and DNA is immobilized. Paper punches can be placed directly in PCR reactions following washing steps to remove weakly bound proteinaceous material. Although this process is not currently adopted due to challenges associated with static electricity, it is promising for future direct amplification applications ${ }^{5}$. 
Invitrogen's ChargeSwitch ${ }^{\circledR}$ DNA extraction mechanism is unique for its $\mathrm{pH}$-dependent nucleic acid binding and elution. These particular magnetic particles bind DNA at a $\mathrm{pH}<6.5$ when the beads are positively charged, attracting the negatively charged DNA molecules and allowing proteins and contaminants to be removed by washing. Once the contaminants are removed, the $\mathrm{pH}$ is increased $>8.5$ which changes the resin to be negatively charged and release the DNA ${ }^{91,96}$. Although ChargeSwitch ${ }^{\circledR}$ is a simple platform for DNA purification, it suffers from significantly lower DNA yields and purity than other extraction platforms and cannot compete with dSPE technology and organic extraction methods ${ }^{97}$.

A unique liquid-based enzymatic method was developed that uses a thermostable proteinase from an Anarctic Bacillus sp. EA1 to yield PCR-ready DNA. To run an assay, a sample is incubated at $75{ }^{\circ} \mathrm{C}$ to allow the enzyme to digest cell membranes and release DNA in a PCR compatible buffer. This is followed by an incubation at $95{ }^{\circ} \mathrm{C}$ which will denature all proteins in the sample. Although this method has been reported with various forms of forensic samples ${ }^{98,99}$, it has not yet been validated for casework. Other commercial methods are available for DNA purification, but have not been reported for forensic samples. These include GeneReleaser ${ }^{\circledR}$ (BioVentures, INC, USA), FlexSTAR+ (AutoGen, USA), and others.

\subsection{DNA Quantitation and Amplification}

Determining the concentration of human DNA in a given sample is critical to ascertain the appropriate sample volume to be utilized in a STR amplification reaction. This is in accordance to the FBI's Quality Assurance Standard 9.4, QAS 2009 because the extraction process can coextract RNA, bacterial, and viral DNA alongside any human genomic DNA. Ideally, most STR amplifications operate within an optimal mass range of $0.5 \mathrm{ng}-2 \mathrm{ng}$ of DNA ${ }^{5}$. Anything higher 
than this range can introduce artifacts in a STR profile which are not easily interpretable. DNA masses below this range will normally result in poor or failed amplification of alleles, starting with longer STR fragments.

A common method for determining the concentration of DNA in a given sample is quantitative PCR (qPCR). The quality and quantity of the DNA is measured in real time as it is amplified using a fluorescent detector to measure the fluorescence from a chelating dye, such as SYBR green, or from a Taqman assay ${ }^{5}$ (see Fig. 9). SYBR green is specific for the detection of double stranded DNA and can be used to detect the formation of PCR amplicons. However, SYBR green is not specific to the target of interest thus there are issues with nonspecific amplification or binding to double stranded co-extracted DNA ${ }^{100}$. For this reason, a Taqman assay is preferred for quantitative DNA measurements. Taqman uses an oligonucleotide probe to track the amplification of target sequences in real time. The probe has a fluorescently tagged reporter molecule on the 5 ' end and a quencher molecule on the 3' end. A fluorescent signal is measured only when the quencher and reporter are not in close proximity to each other ${ }^{100}$. During the annealing stage of PCR, the probe binds to amplified fragments containing the complementary sequence. During extension, the polymerase displaces and physically separates the quencher and reporter molecules leading to an increased fluorescent signal. The fluorescence detector can monitor and record an amplification plot.

The cycle threshold, or PCR cycle number at which there is a significant increase in fluorescence from the background signal, is the parameter used to determine the concentration of samples of interest following qPCR. A standard curve is created and compares the cycle threshold for reference standards with known concentrations. From this curve, the cycle threshold of the unknown sample can estimate the approximate concentration in the original sample. One 
important parameter for DNA quantitation is inclusion of an internal positive amplification control (IPC) that can gauge whether the quantitation assay performed correctly and more importantly whether a quantitative value, assigned to a sample, may have been skewed by inhibition. The HY Plexor kit (Promega) and Quantifiler (Life Technologies) are two commercial available qPCR kits that use an IPC control for DNA quantitation in forensic sample testing.

A number of different instruments are currently used for qPCR assays and include the ABI 7000 series or QuantStudio® instruments (Thermo Fisher Scientific), the iCycler (Bio-Rad), the

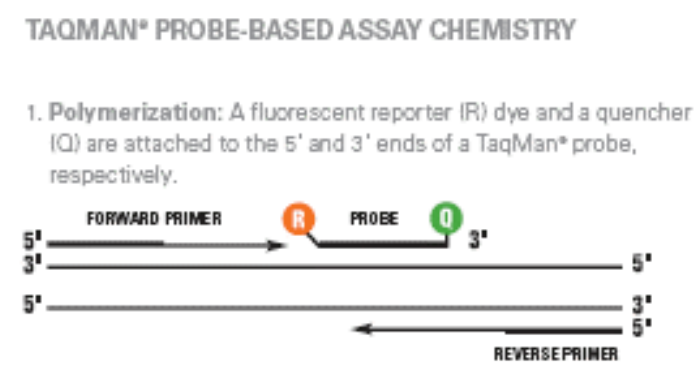

2. Strand displacement: When the probe is intact, the reporter dye emission is quenched.

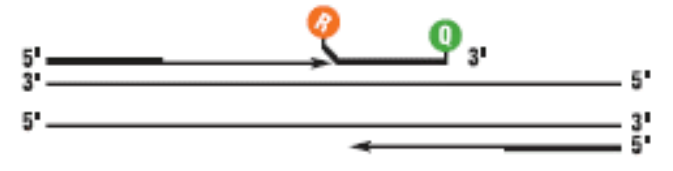

3. Cleavage: During each extension cycle, the DNA polymerase cleaves the reporter dye from the probe.

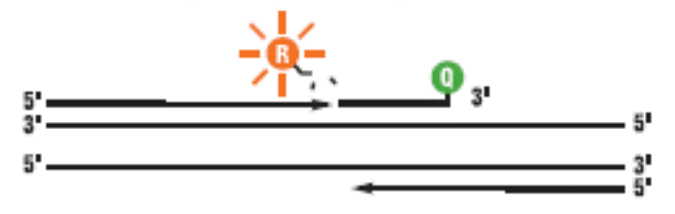

4. Polymerization completed: Once separated from the quencher, the reporter dye emits its characteristic fluorescence.

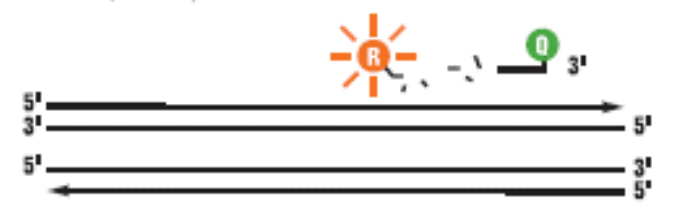

\section{SYBR $^{*}$ GREEN I DYE ASSAY CHEMISTRY}

1. Reaction setup: The SYBR* Green I Dye flucresces when bound to double-stranded DNA.

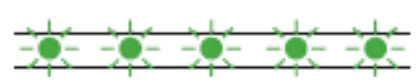

2. Denaturation: When the DNA is denatured, the SYBR* Green I Dye is released and the fluorescence is drastically reduced.

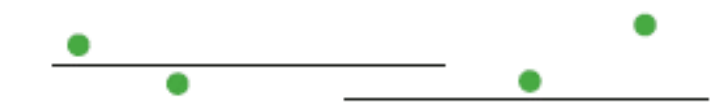

3. Polymerization: During extension, primers anneal and PCA product is generated.

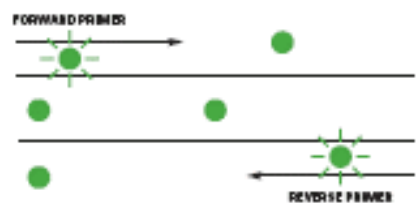

4. Polymerization completed: When polymerization is complete, $\mathrm{SYBR}^{*}$ Green I Dye binds to the double-stranded product, resulting in a net increase in fluorescence detected by the $7900 \mathrm{HT}$ system.

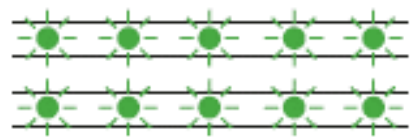

Figure 9: Taqman and SYBR Green Dye comparison for qPCR. Note: polymerization refers to the formation of double stranded DNA via PCR. Adapted from http://www.biosyn.com/tew/taqman-vs-sybr-green-chemistries.aspx. 
LightCycler® (Roche Applied Science), Mx3000P and Mx3005P (Agilent Genomics), and others. The most common instrument for forensic analysis is the ABI 7500 .

Once sample quantitation is completed, appropriate volumes of samples are placed in tubes along with the necessary reagents for amplification. The STR amplification kits amplify and fluorescently tag multiple STR markers simultaneously. Common STR kits, such as AmpF€STR ${ }^{\circledR}$ Identifiler ${ }^{\circledR}$ (Applied Biosystems, USA) and Powerplex ${ }^{\circledR} 16$ HS (Promega, USA) kits target 16 STR loci which include the 13 core loci. With the FBI requirement to include the expanded European loci in 2017, these kits will soon be replaced with STR kits that target 24 loci such as Globalfiler® (Applied Biosystems, USA) and Powerplex ${ }^{\circledR}$ Fusion (Promega, USA).

The newer kits provide increased discrimination for human identification but also come with improved chemistry for more efficient amplification. Buffer advancements, for example, have increased the robustness of STR kits to PCR inhibitors such as hemoglobin or synthetic dyes (Fig. 10). This allows for use of a direct amplification process, in which STR profiles can be obtained from samples without prior extraction or quantitation ${ }^{11}$. Some success has been found in developing direct amplification methods that are compatible with common STR kits ${ }^{11,101-103}$ with the addition of proprietary direct amplification buffers. However, direct amplification is only applicable to single-source samples and requires further research before it can be validated for casework.

\subsection{DNA Separation and Detection}

Capillary electrophoresis (CE) is exclusively used to separate STR samples prior to detection. There are three basic requirements for reliable STR typing with CE: single base pair resolution of fragments, spectral resolution (separating fluorescent dyes), and reproducible sample 
injections ${ }^{5}$. The most commonly used instrument that complies with all requirements is an ABI 3130, which can simultaneously inject up to 16 samples in 45 minutes. Some laboratories have validated an upgraded instrument (ABI 3500) for casework samples, which can analyze up to 24 samples simultaneously in 45 minutes.

Single base pair resolution is necessary to discriminate all possible alleles of all STR markers at the selected loci. As mentioned previously, the number of possible repeats for each STR marker can be highly variable and detection methods need to be able to capture all possible combinations for human identification. One of the 13 core loci, TH01, requires the separation of a 9.3 and a 10 allele, which vary in length by only 1 base pair and are both are commonly found. To achieve single base separation, the capillary length, polymer type and concentration, and voltage must be considered. Thanks to research efforts, companies developing the STR separation instrumentation have achieved single base pair separation with a capillary length of $36 \mathrm{~cm}$ and a performance optimized polymer (POP-4) at a running voltage of $10 \mathrm{kV}^{105}$. Spectral resolution is achieved with a CCD camera and an optimized color matrix that can differentiate fluorescent signals from each of the dyes being used. Manufacturer software allows for simple interpretation

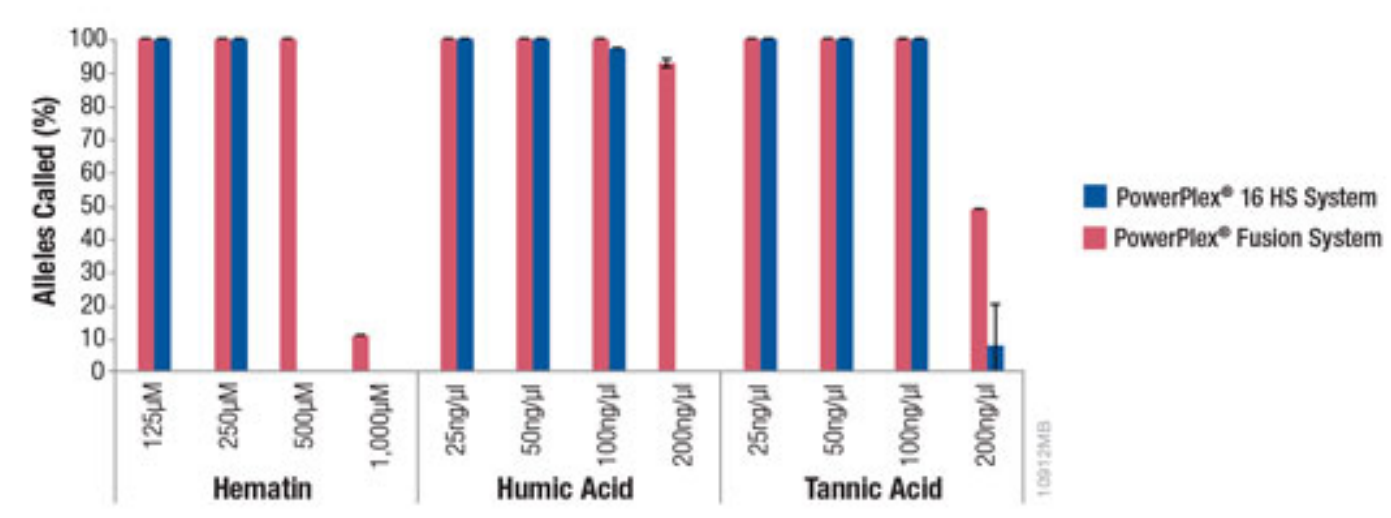

Figure 10: Graphical representation of the improved tolerance to PCR inhibitors with the newer Promega Fusion STR kit ${ }^{104}$. 
of all data following each injection, separation, and detection. Lastly, reproducible spectra are achieved with the simultaneous injection of an allelic ladder, which acts as an internal size standard for each loci under the same separation conditions. This allows accurate assignment of each allele at every loci based on migration time through the capillary (Fig. 11).

\subsection{Integrated Human Identification Systems}

In 2010, the FBI launched a Rapid DNA Program Office for the development of an integrated and automated system that could produce a STR profile from a sample in 90 minutes or less. This effort was instituted to facilitate accelerated forensic results and quicker arrests. Ideally, these instruments were to be kept at police stations to assist in the booking process. Since most of these samples were reference DNA samples, these instruments did not need to include presumptive testing steps.

Although this initiative introduces many challenges, several research groups have shown promising progress towards an integrated system. Liu et al. ${ }^{107}$ developed a fully integrated device capable of extraction, amplification, and separation of reference samples within 3 hours. A nonconventional sequence-specific DNA extraction method was used to specifically capture DNA sequences that matched those used in a STR amplification and concentrate the template DNA prior to amplification. Rapid STR amplification was completed in as little as 40 minutes due to a reduced volume of $250 \mathrm{~nL}$ and a simpler STR assay only targeting 9-loci. With less loci, samples could be detected in 30 minutes with a shortened $14 \mathrm{~cm}$ separation channel. 


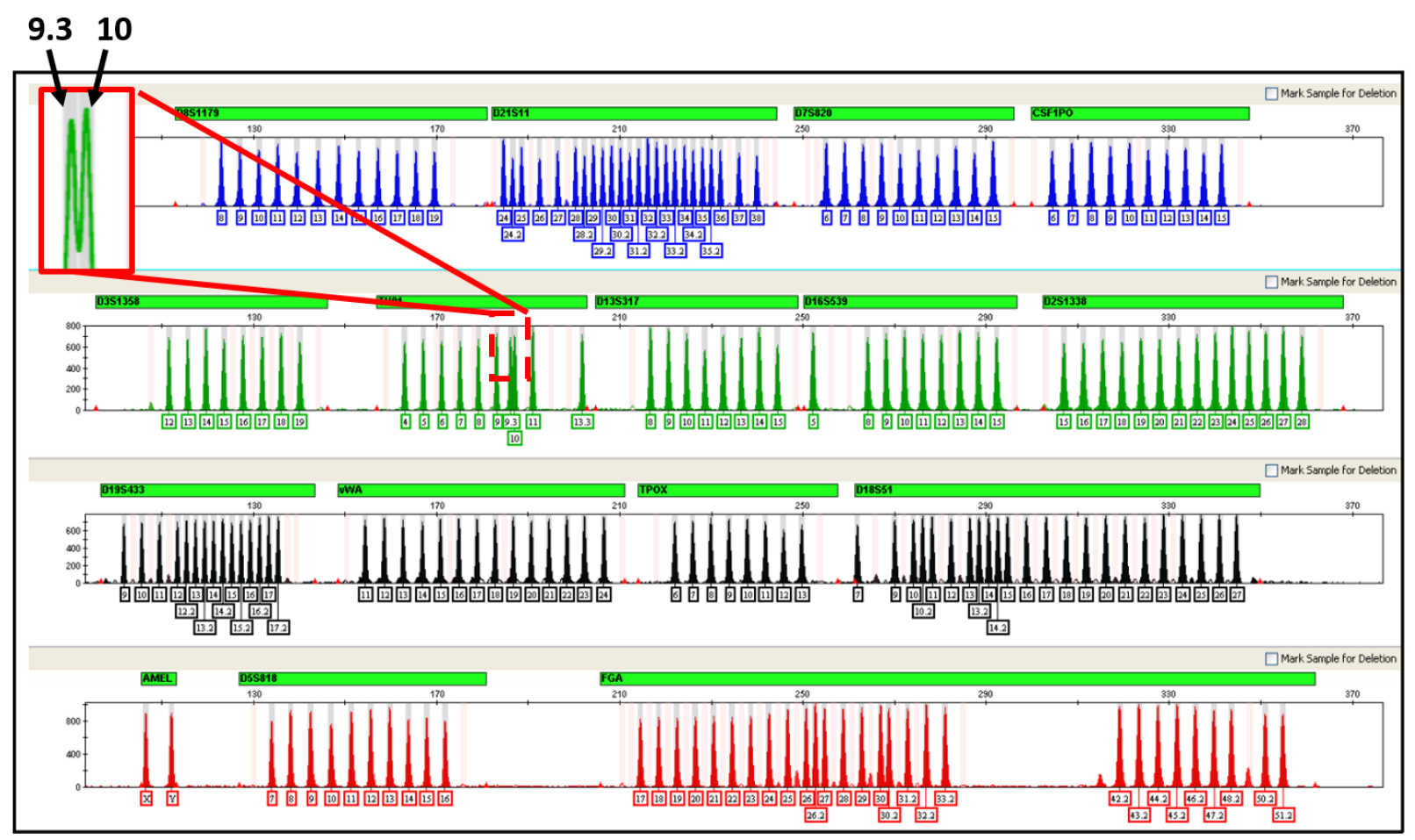

Figure 11: Allelic ladder separation of Identifiler chemistry on an ABI $3130 \mathrm{xl}$ with an expanded region showing the 9,3 and 10 alleles within the TH01 loci ${ }^{106}$.

Another example of an integrated system, from Microlab Diagnostics Inc. can processes up to four buccal swabs simultaneously within 2 hours ${ }^{108}$. The miniaturized device is comparable to the size of a 96 well plate and utilizes on-board reagents for a 'swab in-profile-out' protocol (Fig. 12A). DNA liberation technology coupled with a short $7 \mathrm{~cm}$ separation channel yields full STR profiles with remarkable single base pair resolution (Fig. 12B). Although an IntrepIDS $2 A 90^{\mathrm{TM}}$ instrument was manufactured for full automation of these microdevices ${ }^{109}$, it did not stay in the market long enough to be evaluated for forensic samples. 
The paradigm shift towards miniaturized devices paves the way for achieving portable, robust, and rapid DNA processing for buccal swab or dried blood punch samples. Several commercial instruments are currently being evaluated for the FBI Rapid initiative: RapidHIT® ${ }^{110}$ 111 (IntegenX, USA), Accelerated Nuclear DNA Equipment (ANDE ${ }^{\mathrm{TM}}$ ) instrument ${ }^{12,113}$ (Network Biosystems, USA) and DNAscan ${ }^{\mathrm{TM}}$ Rapid DNA Analysis ${ }^{\mathrm{TM}}$ (Network Biosystems, USA). In April of 2016, the DNAscan ${ }^{\mathrm{TM}}$ system was reportedly given National DNA Index System (NDIS) approval from the FBI which allows the STR profiles from the instrument to be uploaded and matched to STR profiles on databases including CODIS. It will only be a matter of time before
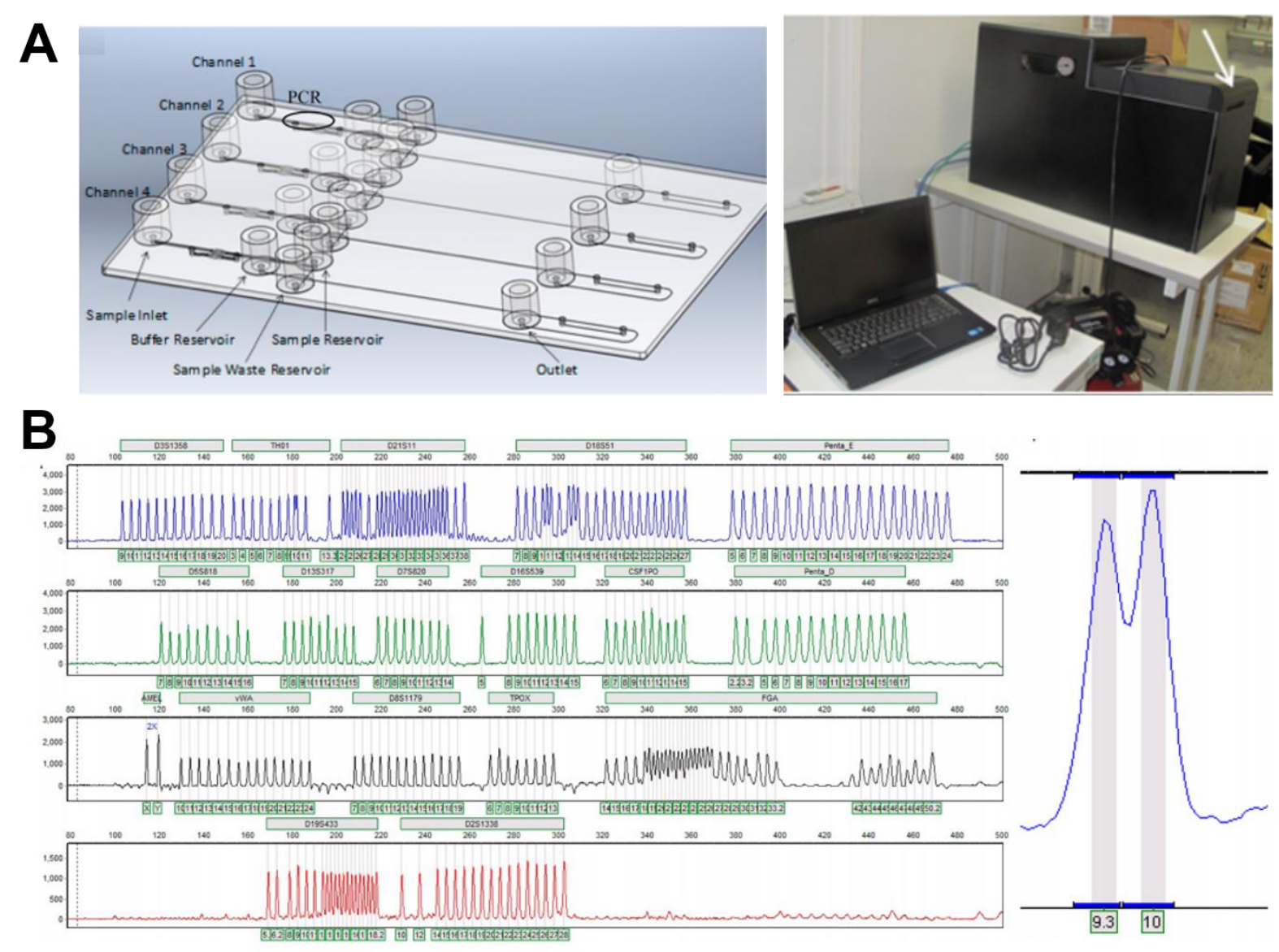

Figure 12: Integrated DNA processing on a microfluidic platform. (A) Microfluidic device that can accommodate 4 buccal swabs fits into the instrument (right) where the white arrow points. (B) Allelic ladder separation on the microfluidic device with a zoomed TH01 region to demonstrate single base resolution ${ }^{108}$. 
these instruments will be placed in forensic labs, deployed overseas for use by military personnel, and in police stations.

\subsection{Concluding Remarks}

These advancements will continue to reduce casework backlogs while increased processing abilities. This dissertation will discuss work towards improving the current DNA processing workflow in an effort towards rapid DNA screening prior to the integration of rapid DNA systems. Chapter 2 introduces the development of a preliminary bead-based screening method that can be used for determining the presence of human genomic DNA. The prescreening method for DNA is then improved and expanded to include the identification of body fluids in Chapter 3. Chapter 4 will introduce the initial efforts of miniaturizing the dSPE extraction process from whole blood samples via centrifugal microfluidics. The integration of sample lysis and extraction along with the inclusion of buccal swab samples on a microfluidic device is discussed in Chapter 5. Finally, conclusions and future applications will be outlines in Chapter 6.

\subsection{References}

1. P. Gill, A. J. Jeffreys and D. J. Werrett, Nature, 1985, 318, 577-579.

2. A. J. Jeffreys, V. Wilson and S. L. Thein, Nature, 1985, 314, 67-73.

3. C.-W. Su, C.-Y. Li, J. C.-I. Lee, D.-D. Ji, S.-Y. Li, B. Daniel, D. Syndercombe-Court, A. Linacre and H.-M. Hsieh, Forensic Science Medicine and Pathology, 2015, 11, 208-215.

4. L. Roewer, Investigative genetics, 2013, 4, 1 .

5. J. Butler, Advanced Topics in Forensic DNA Typing, Academic Press, 2011.

6. R. K. Saiki, S. Scharf, F. Faloona, K. B. Mullis, G. T. Horn, H. A. Erlich and N. Arnheim, Science, $1985,230,1350-1354$. 
7. S. H. Wong and I. Sunshine, Handbook of analytical therapeutic drug monitoring and toxicology, CRC Press, 1996.

8. P. Thanakiatkrai and L. Welch, Forensic Science International: Genetics, 2011, 5, 285-290.

9. B. Budowle, T. R. Moretti, A. L. Baumstark, D. A. Defenbaugh and K. M. Keys, Journal offorensic sciences, 1999, 44, 1277-1286.

10. D. R. Hares, Forensic Science International: Genetics, 17, 33-34.

11. D. E. Hall and R. Roy, Croatian Medical Journal, 2014, 55, 655-661.

12. J. A. Lounsbury, Doctorate of Philosophy in Chemistry, The University of Chemistry, 2012.

13. L. Gefrides and K. Welch, in The forensic laboratory handbook procedures and practice, Springer, 2011, pp. 15-50.

14. S. R. F. Harbison, Research and Reports in Forensic Medical Science, 2016, 2016:6, 11-23.

15. K. Virkler and I. K. Lednev, Forensic Science International, 2009, 188, 1-17.

16. J. M. Romeika and F. Yan, Journal of Forensic Research, 2013, 2014.

17. S. S. Tobe, N. Watson and N. N. Daeid, Journal of forensic sciences, 2007, 52, 102-109.

18. J. L. Webb, J. I. Creamer and T. I. Quickenden, Luminescence, 2006, 21, 214-220.

19. R. E. Gaensslen, Sourcebook in forensic serology, immunology, and biochemistry, US Department of Justice, National Institute of Justice, 1983.

20. D. Petersen and M. Kaplan, Journal of forensic sciences, 2011, 56, 733-735.

21. H. Poon, J. Elliott, J. Modler and C. Fregeau, Journal of forensic sciences, 2009, 54, 1278-1286.

22. M. Vennemann, G. Scott, L. Curran, F. Bittner and S. S. Tobe, Forensic Sci Med Pathol, 2014, 10, 69-75.

23. A. Sinelnikov, A. Kalinina, J. B. Old, P. W. Boonlayangoor and K. A. Reich, Applied Sciences, 2013, 3, 624-635.

24. R. M. Gardner, Practical crime scene processing and investigation, CRC Press, 2011.

25. J.-H. An, K.-J. Shin, W.-I. Yang and H.-Y. Lee, BMB reports, 2012, 45, 545-553.

26. R. Li, Forensic Chemistry Handbook, 2011, 269-290. 
27. A. M. Gross, K. A. Harris and G. L. Kaldun, Journal of forensic sciences, 1999, 44, 837-840.

28. M. Woźniak, M. Papuga, K. Stopińiska and K. Sliwka, Archiwum medycyny sadowej $i$ kryminologii, 2005, 56, 239-245.

29. I. Quinones, D. Sheppard, S. Harbison and D. Elliot, Canadian Society of Forensic Science Journal, 2007, 40, 53-63.

30. S. Johnston, J. Newman and R. Frappier, Canadian Society of Forensic Science Journal, 2003, 36, 173-183.

31. A. Durdle, R. J. Mitchell and R. A. Oorschot, Journal of forensic sciences, 2015, 60, 468-470.

32. B. A. Schweers, J. Old, P. W. Boonlayangoor and K. A. Reich, Forensic Science International: Genetics, 2008, 2, 243-247.

33. R. Saferstein, Criminalistics: An Introduction to Forensic Science, Prentice Hall, 7th edn., 2001.

34. R. C. Shaler, Forensic Science Handbook, Prentice Hall, Upper Saddle River, NJ, 2002, 529-546.

35. M. M. Hobbs, M. J. Steiner, K. D. Rich, M. F. Gallo, A. Alam, M. Rahman, P. Menezes, T. Chipato, L. Warner and M. Macaluso, Sexually transmitted diseases, 2009, 36, 501-506.

36. E. Diamandis and H. Yu, The Lancet, 1995, 345, 1186.

37. R. Nagar and K. K. Sharma, Indian Journal of Clinical Biochemistry, 2012, 27, 375-378.

38. P. Lunetta and H. Sippel, Journal of Forensic and Legal Medicine, 2009, 16, 397-399.

39. L. A. Christel, K. Petersen, W. McMillan and M. A. Northrup, Journal of Biomechanical Engineering-Transactions of the Asme, 1999, 121, 22-27.

40. Á. Laffan, I. Sawyer, I. Quinones and B. Daniel, Medicine, Science and the Law, 2011, 51, 11-17.

41. J. Herr, SpermPaint optimization and validation, publisher not identified, 2007.

42. A. De Moors, T. Georgalis, G. Armstrong, J. Modler and C. J. Frégeau, Forensic science international: genetics, 2013, 7, 367-379.

43. D. L. Laux, A. J. Tambasco and E. A. Benzinger, Journal, 2006.

44. T. R. De Wolff, A. J. Kal, C. E. H. Berger and B. Kokshoorn, Law, Probability and Risk, 2015, 14, 323-339. 
45. J. R. Myers and W. K. Adkins, Journal of forensic sciences, 2008, 53, 862-867.

46. B. Pang and B. K. Cheung, Journal of forensic sciences, 2008, 53, 1117-1122.

47. D. G. Casey and J. Price, Forensic Science International, 2010, 194, 67-71.

48. U. Ricci, I. Carboni and F. Torricelli, Journal of forensic sciences, 2014, 59, 1410-1412.

49. E. Hanson and J. Ballantyne, Forensic science review, 2010, 22, 145-157.

50. E. K. Hanson and J. Ballantyne, F1000Research, 2013, 2.

51. D. Zubakov, E. Hanekamp, M. Kokshoorn, W. van IJcken and M. Kayser, International journal of legal medicine, 2008, 122, 135-142.

52. M. Setzer, J. Juusola and J. Ballantyne, Journal of forensic sciences, 2008, 53, 296-305.

53. M. Bauer and D. Patzelt, Forensic science international, 2003, 136, 76-78.

54. C. Haas, E. Hanson, M. Anjos, W. Bär, R. Banemann, A. Berti, E. Borges, C. Bouakaze, A. Carracedo and M. Carvalho, Forensic Science International: Genetics, 2012, 6, 70-80.

55. A. Bowden, R. Fleming and S. Harbison, Forensic Science International: Genetics, 2011, 5, 6468.

56. J.-L. Park, S.-M. Park, J.-H. Kim, H.-C. Lee, S.-H. Lee, K.-M. Woo and S.-Y. Kim, Genomics Inform, 2013, 11, 277-281.

57. A. D. Roeder and C. Haas, International journal of legal medicine, 2013, 127, 707-721.

58. P. Danaher, R. L. White, E. K. Hanson and J. Ballantyne, Forensic Science International: Genetics, $2015,14,18-30$.

59. C. Haas, E. Hanson, A. Kratzer, W. Bär and J. Ballantyne, Forensic Science International: Genetics, 2011, 5, 449-458.

60. إبـ راهيم .م .ت and The Egyptian Journal of Forensic Sciences and Applied Toxicology, 2012, 12, 27-46.

61. S.-M. Park, S.-Y. Park, J.-H. Kim, T.-W. Kang, J.-L. Park, K.-M. Woo, J.-S. Kim, H.-C. Lee, S.Y. Kim and S.-H. Lee, Forensic Science International: Genetics, 2013, 7, 143-150.

62. J. Donfack and A. Wiley, Forensic Science International: Genetics, 2015, 16, 112-120. 
63. M. Ha and V. N. Kim, Nature reviews Molecular cell biology, 2014, 15, 509-524.

64. E. Hanson, H. Lubenow and J. Ballantyne, Forensic Science International: Genetics Supplement Series, 2009, 2, 503-504.

65. X. Luo, Z. Li, D. Peng, L. Wang, L. Zhang and W. Liang, Forensic Science International: Genetics Supplement Series, 2015, 5, e630-e632.

66. D. Zubakov, A. W. Boersma, Y. Choi, P. F. van Kuijk, E. A. Wiemer and M. Kayser, International journal of legal medicine, 2010, 124, 217-226.

67. C. Courts and B. Madea, Journal of forensic sciences, 2011, 56, 1464-1470.

68. Z. Wang, J. Zhang, H. Luo, Y. Ye, J. Yan and Y. Hou, Forensic Science International: Genetics, 2013, 7, 116-123.

69. J. L. Park, S. M. Park, O. H. Kwon, H. c. Lee, J. y. Kim, H. H. Seok, W. S. Lee, S. H. Lee, Y. S. Kim and K. M. Woo, Electrophoresis, 2014, 35, 3062-3068.

70. F. Eckhardt, J. Lewin, R. Cortese, V. K. Rakyan, J. Attwood, M. Burger, J. Burton, T. V. Cox, R. Davies and T. A. Down, Nature genetics, 2006, 38, 1378-1385.

71. J. P. Antunes, T. Madi, K. Balamurugan, R. Bombardi, G. Duncan and B. McCord, 2014.

72. H. Y. Lee, M. J. Park, A. Choi, J. H. An, W. I. Yang and K.-J. Shin, International journal of legal medicine, 2012, 126, 55-62.

73. E. Kitamura, J. Igarashi, A. Morohashi, N. Hida, T. Oinuma, N. Nemoto, F. Song, S. Ghosh, W. A. Held and C. Yoshida-Noro, Genomics, 2007, 89, 326-337.

74. R. Illingworth, A. Kerr, D. DeSousa, H. Jørgensen, P. Ellis, J. Stalker, D. Jackson, C. Clee, R. Plumb and J. Rogers, PLoS Biol, 2008, 6, e22.

75. S. Forat, B. Huettel, R. Reinhardt, R. Fimmers, G. Haidl, D. Denschlag and K. Olek, PLoS ONE, 2016, 11, e0147973.

76. J. H. An, A. Choi, K.-J. Shin, W. I. Yang and H. Y. Lee, International journal of legal medicine, 2013, 127, 35-43. 
77. D. Frumkin, A. Wasserstrom, B. Budowle and A. Davidson, Forensic Science International: Genetics, 2011, 5, 517-524.

78. C. Robino, S. Gino and C. Torre, 2004.

79. B. L. LaRue, J. L. King and B. Budowle, International journal of legal medicine, 2013, 127, 299308.

80. T. Akutsu, H. Motani, K. Watanabe, H. Iwase and K. Sakurada, Legal Medicine, 2012, 14, 160162.

81. C. C. Benschop, F. C. Quaak, M. E. Boon, T. Sijen and I. Kuiper, International journal of legal medicine, 2012, 126, 303-310.

82. H. Nakanishi, T. Ohmori, M. Hara, A. Takada, H. Shojo, N. Adachi and K. Saito, Journal of forensic sciences, 2011, 56.

83. S. Giampaoli, A. Berti, F. Valeriani, G. Gianfranceschi, A. Piccolella, L. Buggiotti, C. Rapone, A. Valentini, L. Ripani and V. R. Spica, Forensic Science International: Genetics, 2012, 6, 559-564.

84. A. Choi, K.-J. Shin, W. I. Yang and H. Y. Lee, International Journal of Legal Medicine, 2014, 128, $33-41$.

85. P. Iadarola, M. Fumagalli, A. M. Bardoni, R. Salvini and S. Viglio, Electrophoresis, 2016, 37, 212230.

86. K. M. Legg, R. Powell, N. Reisdorph, R. Reisdorph and P. B. Danielson, Electrophoresis, 2014, 35, 3069-3078.

87. E. Cappellini, L. J. Jensen, D. Szklarczyk, A. Ginolhac, R. A. da Fonseca, T. W. Stafford Jr, S. R. Holen, M. J. Collins, L. Orlando and E. Willerslev, Journal of proteome research, 2011, 11, 917926.

88. R. D. Smith, Clinical chemistry, 2012, 58, 528-530.

89. H. Yang, B. Zhou, H. Deng, M. Prinz and D. Siegel, International journal of legal medicine, 2013, 127, $1065-1077$. 
90. K. Van Steendam, M. De Ceuleneer, M. Dhaenens, D. Van Hoofstat and D. Deforce, International Journal of Legal Medicine, 2013, 127, 287-298.

91. C. W. Price, D. C. Leslie and J. P. Landers, Lab on a Chip, 2009, 9, 2484-2494.

92. B. Vogelstein and D. Gillespie, Proceedings of the National Academy of Sciences of the United States of America, 1979, 76, 615-619.

93. (ed. 04/2010), Available online at http://www.qiagen.com.

94. J. C. B. K.R. Jackson, M. Meija, B.L. Poe, E. Carrilho, D.M. Haverstick, and J.P. Landers, San Antonio, Texas, 2014.

95. M. Stangegaard, C. Børsting, L. Ferrero-Miliani, R. Frank-Hansen, L. Poulsen, A. J. Hansen and N. Morling, Journal of laboratory automation, 2013, 18, 404-410.

96. W. D. Cao, C. J. Easley, J. P. Ferrance and J. P. Landers, Anal. Chem., 2006, 78, 7222-7228.

97. A. Psifidi, C. I. Dovas, G. Bramis, T. Lazou, C. L. Russel, G. Arsenos and G. Banos, PloS one, 2015, 10, e0115960.

98. J. A. Lounsbury, N. Coult, D. C. Miranian, S. M. Cronk, D. M. Haverstick, P. Kinnon, D. J. Saul and J. P. Landers, Forensic Science International: Genetics, 2012, 6, 607-615.

99. B. L. Thompson, C. Birch, J. Li, J. A. DuVall, D. Le Roux, D. A. Nelson, A.-C. Tsuei, D. L. Mills, S. T. Krauss, B. E. Root and J. P. Landers, Analyst, 2016, DOI: 10.1039/C6AN00209A.

100. T. Scientific, TaqMan® Chemistry vs. SYBR® Chemistry for Real-Time PCR, https://www.thermofisher.com/us/en/home/life-science/pcr/real-time-pcr/qpcr-education/taqmanassays-vs-sybr-green-dye-for-qpcr.html\#taqman).

101. D. Y. Wang, C. W. Chang, R. E. Lagace, L. M. Calandro and L. K. Hennessy, Journal of Forensic Sciences, 2012, 57, 453-465.

102. A. Linacre, V. Pekarek, Y. C. Swaran and S. S. Tobe, Forensic science international: genetics, 2010, 4, 137-141.

103. B. A. Myers, J. L. King and B. Budowle, Forensic Science International Genetics, 2012, 6, 640645. 
104. Promega, Powerplex Fusion Systems, http://www.promega.com/products/genetic-identity/stramplification/powerplex-fusion-str-kits/).

105. A. Biosystems.

106. A. Biosystems, 2012.

107. P. Liu, X. Li, S. A. Greenspoon, J. R. Scherer and R. A. Mathies, Lab on a Chip, 2011, 11, 10411048.

108. D. Le Roux, B. E. Root, J. A. Hickey, O. N. Scott, A. Tsuei, J. Li, D. J. Saul, L. Chassagne, J. P. Landers and P. de Mazancourt, Lab on a Chip, 2014, 14, 4415-4425.

109. J. G. Shewale and R. H. Liu, Forensic DNA Analysis: Current Practices and Emerging Technologies, CRC Press, 2013.

110. S. Verheij, L. Clarisse, M. van den Berge and T. Sijen, Forensic Science International: Genetics Supplement Series, 2013, 4, e254-e255.

111. S. Jovanovich, G. Bogdan, R. Belcinski, J. Buscaino, D. Burgi, E. L. Butts, K. Chear, B. Ciopyk, D. Eberhart and O. El-Sissi, Forensic Science International: Genetics, 2015, 16, 181-194.

112. R. S. Turingan, S. Vasantgadkar, L. Palombo, C. Hogan, H. Jiang, E. Tan and R. F. Selden, Investigative Genetics, 2016, 7, 1-12.

113. E. Tan, R. S. Turingan, C. Hogan, S. Vasantgadkar, L. Palombo, J. W. Schumm and R. F. Selden, Investigative Genetics, 2013, 4, 1-15. 


\section{Characterization of High Affinity Aggregation for Detection of Targeted Amplicons}

\section{Summary}

In this chapter, the development of a novel sequence-specific high affinity aggregation (HAA) method is described for rapid and sensitive detection of DNA and RNA target sequences. HAA exploits the high affinity between streptavidin coated beads and biotinylated sequences to induce bead aggregation. Unlike other previously developed bead detection methods, HAA is only limited by the restraints of the amplification method. Using loop-mediated isothermal amplification (LAMP), a detection range nearing single copies of DNA (20 pg) up through $100 \mathrm{ng}$ was achieved. Broad applicability of HAA was demonstrated with clinical genotyping of Warfarin using single-nucleotide polymorphisms, Rift Valley fever virus (RVFV) for bio warfare agent detection, and Salmonella enterica for foodborne pathogen detection. This chapter also describes quantitative qualities of the HAA assay for possible real-time amplification detection

\subsection{Introduction}

Molecular diagnostics rely on rapid, simple detection methods for fields ranging from pathogen detection to clinical diagnostics. Polymerase chain reaction (PCR) is conventionally used with fluorescence detection due to PCR sensitivity, detection bandwidth, and speed of detection. However, PCR is sensitive to the presence of inhibitors (i.e., bile salts and humic acids) and optimization can be complex. In addition, fluorescence detection is expensive and limited due to the optical specifications necessary for detection. The need for a robust, rapid, and simple amplification and detection method led to the exploration of loop-mediated isothermal amplification (LAMP). 
LAMP was originally reported in $2000^{2}$ and uses a set of 4-6 primers for high-specificity amplification. These primers include two inner primers, FIP (composed of a F1C and F2 sequence), BIP (composed of a B1C and B2 sequence), and two outer primers, F3 and B3. The locations for these primer binding sites are shown in Figure 1A, which include F3, F2, F1C, $\mathrm{B} 1 \mathrm{C}, \mathrm{B} 2$, and $\mathrm{B} 3$ from the 5 ' end. In the LAMP preparation stage, all primers bind alongside the target sequence to form a 'stem-loop' structure prior to amplification. If one of these primer sequences does not bind, then amplification will not occur. To form the 'stem-loop', the FIP primer first binds to F2C site on the DNA and initiates elongation (Figure 1B). The F3 primer then anneals to the target sequence and catalyzes a single strand displacement of the elongated DNA. The released DNA strand will form a loop at the 5' end, as F1 is complementary to F1c (structure 4 in Figure 1B). This two-step process repeats itself with the BIP primer followed by single strand displacement via B3. As B1 is complementary to B1c, a second loop will form at the 3' end, leaving a 'stem-loop' structure that can be amplified.

Once a 'stem-loop' structure has formed (structure 5), the process moves into an amplification stage where only the FIP and BIP primers are active (Fig 1C). This process begins with the annealing of the FIP primer at the F2 site (structure 5, Fig. 1C) followed by the annealing of a BIP primer. This will create an amplification cycle where the 'stem-loop' structure will switch between the normal 'stem-loop' to a complementary 'stem-loop' structure (structure 7). Elongated products (structures 9 and 11) will be formed from intermediate steps in this cycle and include structures 6 and 8. This will provide LAMP amplicons of various sizes.

The LAMP process can be accelerated with the use of two loop primers, LF and LB. These loop primers are active in the amplification stage of LAMP and hybridize to the outer stem loops while allowing simultaneous amplification of the FIP and BIP inner primers. Nagamine et. 

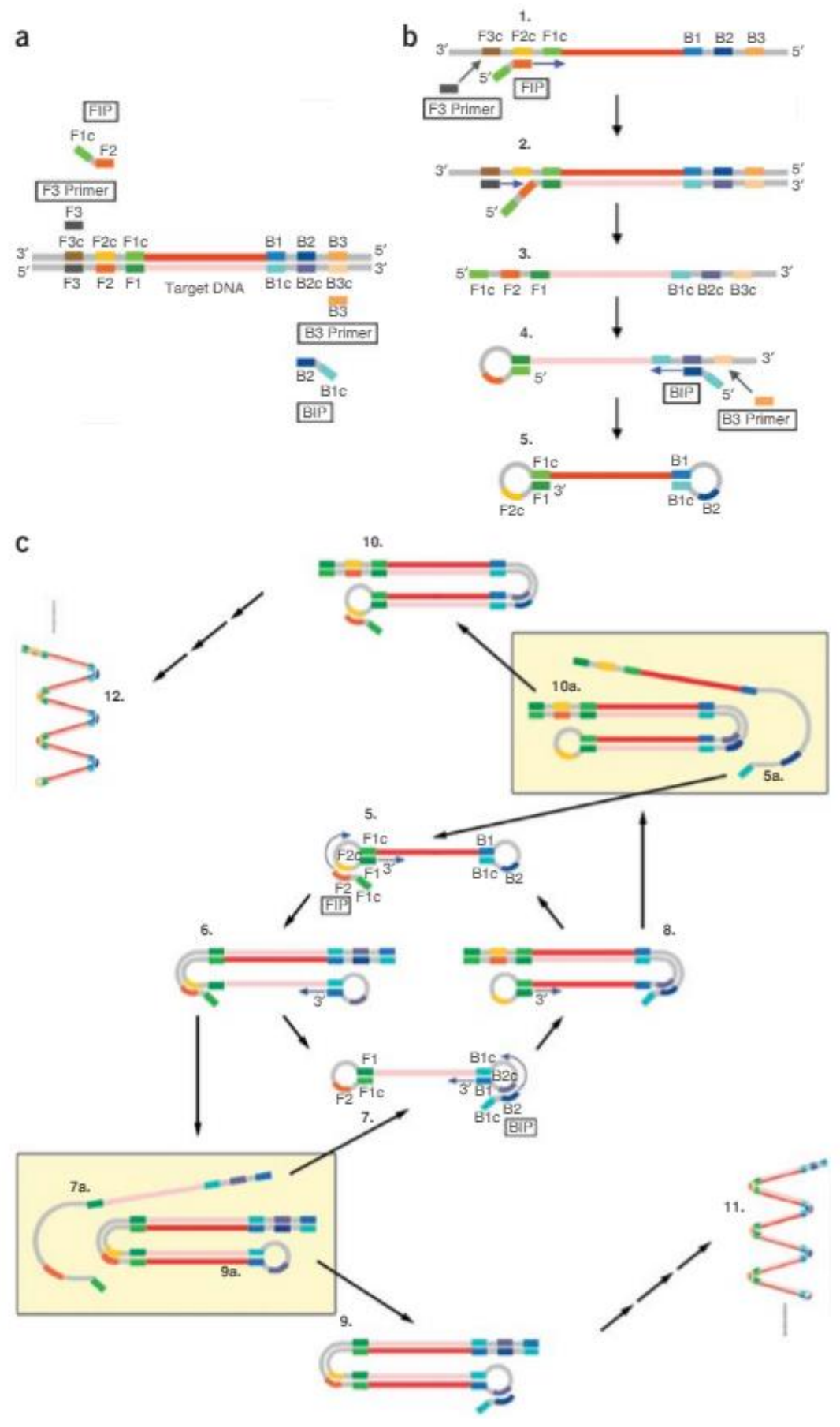

Figure 1: Principle for LAMP Amplification. (A) Illustration of the binding sites along a target sequence for LAMP. (B) Process for formation of a 'stem-loop' structure prior to the amplification stage of LAMP. (C) Process for LAMP amplification from the 'stem-loop' structure. Adapted from Tomita et. al 2008. 
al found that amplification with loop primers can decrease the amplification by $50 \%$ due to accelerated amplification ${ }^{3}$. Since then, most LAMP studies have adopted loop primers for rapid amplification $^{4-6}$.

LAMP has many advantages over traditional PCR such as increased specificity, single temperature amplification, and a decreased amplification time of 60 minutes ${ }^{1,2}$. The increased primers in LAMP to recognize a target of interest provides increased specificity for amplifying difficult samples such as specific strains of bacteria ${ }^{7-9}$ or single nucleotide polymorphisms ${ }^{10-12}$. LAMP also produces a significant amount of magnesium pyrophosphate during amplification that is exploited for fluorescence detection using calcein, direct turbidity measurements, and absorbance measurements using a colorimetric dye $\mathrm{e}^{1,13}$. All of these detection methods, although useful, require bulky instrumentation and large volumes (minimum of $25 \mu \mathrm{L}$ ) for reliable analytical measurements. Instead, bead detection platforms could be used for simple, accurate measurements of LAMP products.

Bead detection platforms have been studied over the last 20 years ${ }^{14-16}$ and can accurately detect analytes from 1-2 $\mu \mathrm{L}$ of sample. One of these methods, product inhibited bead aggregation (PIBA), has already demonstrated sensitive detection of LAMP products and is based on the well-known entropically-driven binding of DNA to silica in the presence of a high concentration guanidine solution ${ }^{7,17}$. In the presence of a rotating magnetic field, these LAMP amplicons coat the surface of the magnetic particles, thus preventing 'trigger DNA', or DNA that would induce aggregation under normal chaotropic conditions, from aggregating beads together. $\mathrm{PiBA}$ is quantitative and highly specific but may only operate at lower concentrations $(<10$ $\mathrm{ng} / \mu \mathrm{L}$ ), as unamplified starting template could compete with LAMP amplicons for sites on the 
particles. As a result, the binding of the template material could mask the inhibition of aggregation with increased concentration and provide false results.

As PiBA has only been studied at $<5 \mathrm{ng} / \mu \mathrm{L}$ starting template with bacterial and human genomic $\mathrm{DNA}^{7}$, the upper detection limit is unknown. In this present study, we will define the limitations of PiBA and then propose an alternative bead detection method platform to PiBA that can more accurately identify target analytes in samples.

\subsection{Materials and Methods}

\subsubsection{Sample Preparation}

Four primary samples were used for this study: male pre-purified human genomic DNA (Promega; Madison, WI) whole blood, salmonella, and Rift Valley Fever virus (RVFV). Whole blood samples were provided from the University of Virginia Medical School. DNA from the blood samples were extracted using a QIAamp DNA mini kit (Qiagen, Germantown, MD) according to the manufacturer protocol. Salmonella enterica (ATCC; Manassas, Virginia) was purchased directly from the manufacturer and used directly for amplification. Lastly, RVFV was prepared as described in DuVall et al. ${ }^{7}$. Briefly, pre-purified RVFV RNA underwent reversetranscription polymerase chain reaction (RT PCR) according to manufacturer instructions (product P/N 4387406; Applied Biosystems; Foster City, CA) and then stored at $-20^{\circ} \mathrm{C}$ until needed for amplification.

\subsubsection{LAMP Amplification}

All LAMP primer sequences were taken from literature ${ }^{7,10,18}$ and directly purchased from Eurofins MWG Operon (Huntsville, AL). To run on the HAA system, all FIP and BIP primer sets 
modified with 5' Biotin. All LAMP amplifications were run according to previously optimized temperatures according to the following resources: Salmonella enterica ${ }^{7}\left(63^{\circ} \mathrm{C}\right), \mathrm{RVFV}^{7}\left(61^{\circ} \mathrm{C}\right)$, Amelogelin $\mathrm{Y}^{18}\left(63{ }^{\circ} \mathrm{C}\right) \mathrm{CYP} 2 \mathrm{C} 19 * 2$ mut and wild-type allele ${ }^{10}\left(60{ }^{\circ} \mathrm{C}\right)$. The primer concentrations for each assay were 5 pmol of F3 and B3 each, 20 pmol each LF and LB, and 40 pmol each of FIP and BIP. A Loopamp DNA amplification kit (Eiken Chemical Co., Ltd, Tokyo, Japan) was used for all amplifications according to the manufacturer instructions. One microliter of each sample was added to a final volume of $12.5 \mu \mathrm{L}$ reaction volume (half reaction).

\subsubsection{Microdevice Fabrication}

Each microfluidic device ( $4 \mathrm{~cm} \times 4 \mathrm{~cm} \times 1.5 \mathrm{~mm}$ ) was designed in AutoCAD software (Autodesk Inc., San Rafael, CA) and fabricated via laser-ablation with a 50 Watt $\mathrm{CO}_{2}$ laser (Universal Laser Systems, Scotsdale, AZ; VLS3.50) followed by thermal bonding. A previously optimized thermal bonding method ${ }^{19}$ was used to thermally seal the laser-ablated layer (1.0 mm thick) with a bottom layer of PMMA (0.5 mm thick). Each device, following fabrication, has 12microwells in a circular array with each holding a volume of approximately $20 \mu \mathrm{L}$.

\subsubsection{Instrument Setup}

The instrument for PiBA and HAA assays are the same and was originally described by Nelson et $\mathrm{al}^{16}$. Briefly, the agitation platform consists of a rotating magnet, fixed above a vortexer (MS3 basic vortexer, IKA, Wilmington, NC) that contains the PMMA device to provide gentile agitation for particle suspension and separation of the beads during magnetic mixing. 


\subsubsection{PiBA Platform}

Magnesil® paramagnetic particles (MP Chemicals, Santa Ana, CA) were diluted 100fold in a pre-made $6 \mathrm{M}$ Guanidine Hydrochloride $(\mathrm{GuHCl}$; $\mathrm{pH} 6.1)$ solution as previously described $^{7}$. To run a PiBA assay, each microwell of a PMMA device was filled with the following: $4 \mu \mathrm{L}$ Magnesil ${ }^{\circledR}$ bead solution, $13 \mu \mathrm{L}$ of $\mathrm{GuHCl}$ solution, $0.5 \mu \mathrm{L}$ of $1 \mathrm{ng} / \mu \mathrm{L}$ pre-purified human genomic DNA ("trigger DNA"; Promega, Madison, WI), and $2 \mu \mathrm{L}$ of a LAMP amplified sample. Once all reagents were loaded, the vortexer was set to $\sim 550$ revolutions per minute (RPM) and a rotating magnetic field at 2200 RPM for 5 minutes. Images of each microwell were taken following the agitation and analyzed in a previously written Mathematica algorithm.

\subsubsection{HAA Platform}

Preliminary experiments for HAA were run with optimized parameters from a previously described streptavidin bead hybridization detection method ${ }^{12}$. To find optimal parameters for HAA, MyOne Streptavidin C1 beads (Invitrogen, Oslo, Norway) were prepared to the following concentrations according to manufacturer instructions: $10 \mathrm{mg} / \mathrm{mL}, 7 \mathrm{mg} / \mathrm{mL}, 5 \mathrm{mg} / \mathrm{mL}, 4.5$ $\mathrm{mg} / \mathrm{mL}$, and $4 \mathrm{mg} / \mathrm{mL}$. A hybridization buffer, composed of $400 \mathrm{mM} \mathrm{KCl}$ and $20 \mathrm{mM}$ Tris (pH 7.5) was also prepared for optimal hybridization between biotin products and streptavidin particles.

To run a HAA assay, a PMMA device was placed on the agitation platform. For each sample of interest, $18 \mu \mathrm{L}$ of the hybridization buffer, $1 \mu \mathrm{L}$ of the prepared particle solution, and 1 $\mu \mathrm{L}$ of a LAMP product were added to a microwell alongside a positive control, negative control, and a "blank" (containing only buffer and beads). Once all reagents were loaded, the beads were mixed in each well with the vortexer, set to 150 revolutions per minute (RPM) and a rotating 
magnetic field (2,000 RPM). Mixing times of 5, 7, 10, and 12 minutes were tested to determine maximum discrimination of a positive and negative sample.

\subsubsection{Data Analysis}

Once bead mixing had completed, digital photos were taken of each well on the PMMA device using a Tli DSLR camera with MP-E 65 mm f/2.8 1-5x macro lens (Cannon U.S.A., Inc., Lake Success, NY). These samples were run through a custom Kapur Mathematica (Champaign, IL) algorithm ${ }^{14}$, for PiBA systems or a modified algorithm (a 150 saturation threshold) for HAA samples.

\subsection{Results and Discussion}

\subsubsection{Defining PiBA Limitations}

Thus far, PiBA studies have focused on defining the advantages of the detection method,

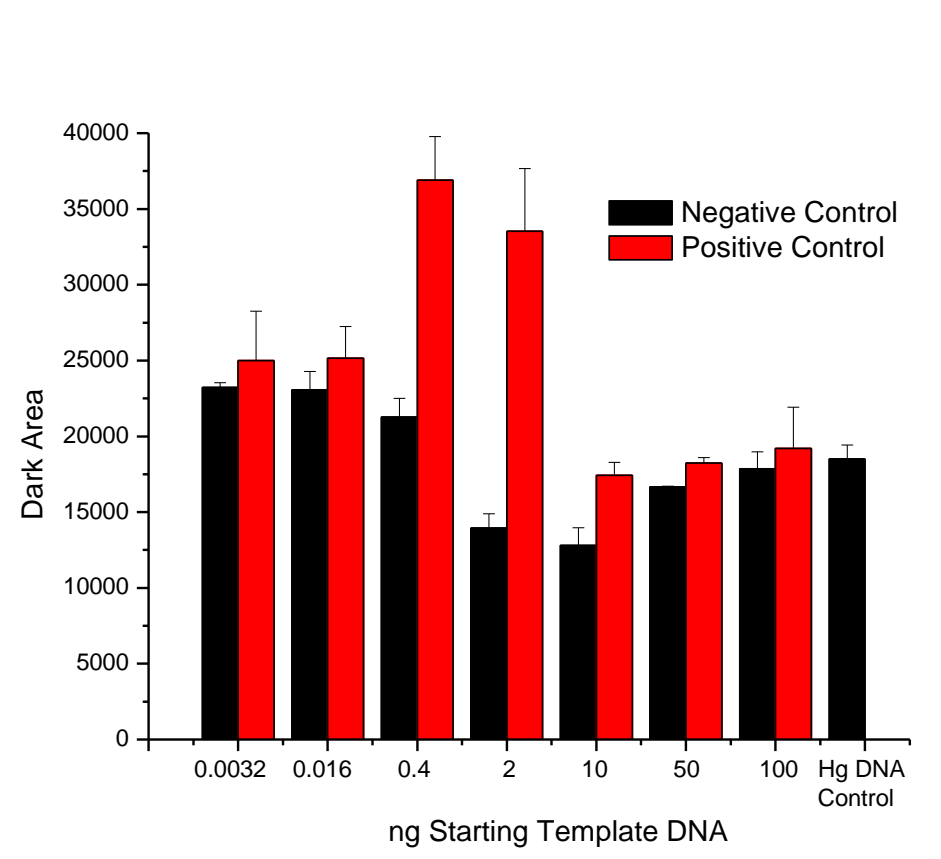

Figure 2: Comparison of the aggregation between positive and negative control samples at increasing concentrations of DNA $(n=3)$. including high specificity and sensitivity, rather than the limitations of the system. We know from the mechanism of PiBA that the LAMP amplicons prevent exogenous DNA from binding to the silica beads within the microwell. However, what is unclear is whether the starting template will mask the amplicon inhibition with increased sample 
A

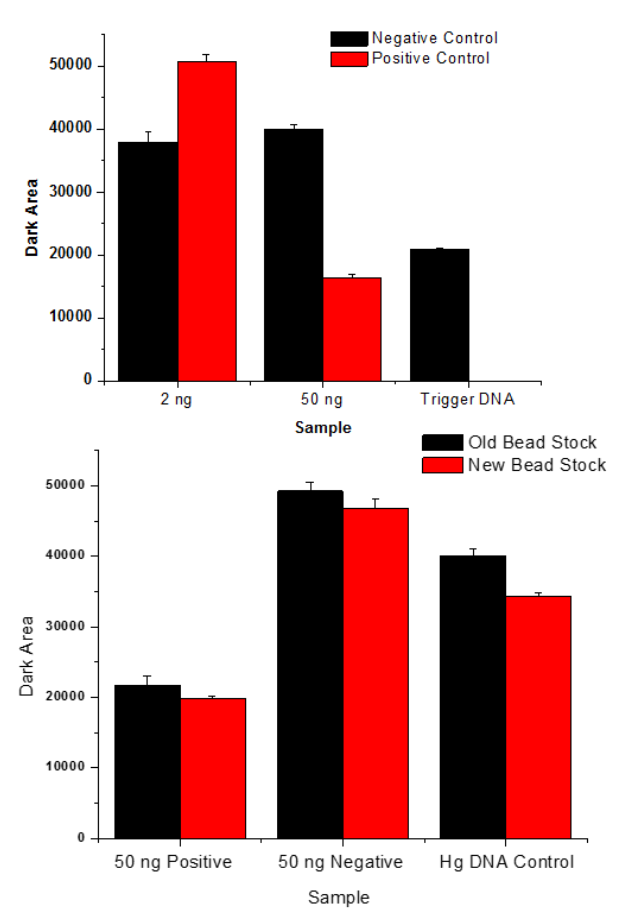

B

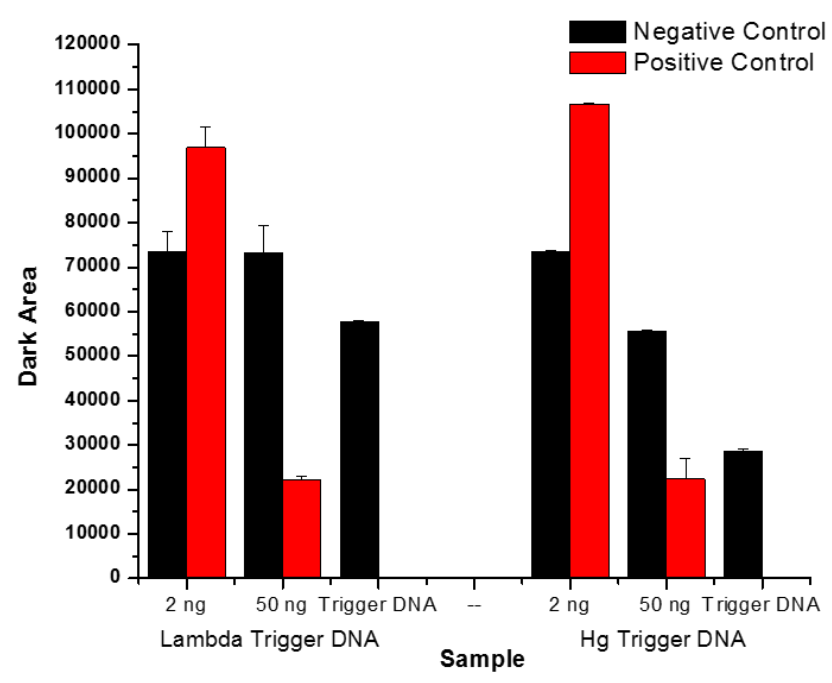

Figure 3: Troubleshooting PiBA Studies. (A) PiBA data $(n=3)$ with the addition of $95^{\circ} \mathrm{C}$ denature step ( 2 min incubation)(top) or the exchange for a new silica bead stock (bottom). (B) Supporting PiBA experiment $(n=3)$ with the exchange of trigger DNA from $1 \mathrm{ng}$ Lambda DNA to $1 \mathrm{ng}$ human genomic (Hg) DNA._It should be noted that positive controls are known DNA target samples and negative controls samples contain only amplification reagents with a Tris-EDTA (TE) buffer solution.

concentrations (> $5 \mathrm{ng}$ DNA). To test this theory, human genomic DNA was amplified via a human-specific DNA LAMP assay ${ }^{18}$ with the following amount of starting template DNA: $3.2 \mathrm{pg}$, $16 \mathrm{pg}, 400 \mathrm{pg}, 2 \mathrm{ng}, 10 \mathrm{ng}, 50 \mathrm{ng}$, and $100 \mathrm{ng}$. These samples were then placed on the agitation platform with optimized PiBA parameters and analyzed with a previously described algorithm ${ }^{14}$ to measure sample aggregation. Aggregation is measured by a 'dark area', which correlates to the total number of pixels occupied by the dark beads. Thus, an increasing dark area is indicative of decreasing bead aggregation. As shown in Figure 2, the $3.2 \mathrm{pg}$ and $16 \mathrm{pg}$ starting DNA template samples did not amplify the target LAMP sequence within 60 minutes, which supported the previously reported lower limits of the LAMP assay ${ }^{7}$. The expected inhibition of the aggregation was observed at both 0.4 and $2 \mathrm{ng}$ starting templates, however, the inhibited response dissipated 
at concentrations equal or greater than 10 ng. To verify this result, the experiment was repeated for $2 \mathrm{ng}$ and $50 \mathrm{ng}$ starting templates with varying reagent changes including new bead stock solutions and varying the 'trigger DNA' (1 $\mathrm{ng}$ तDNA vs human genomic DNA) (see Fig. 3).

Despite these changes, all $50 \mathrm{ng}$ samples remained aggregated and

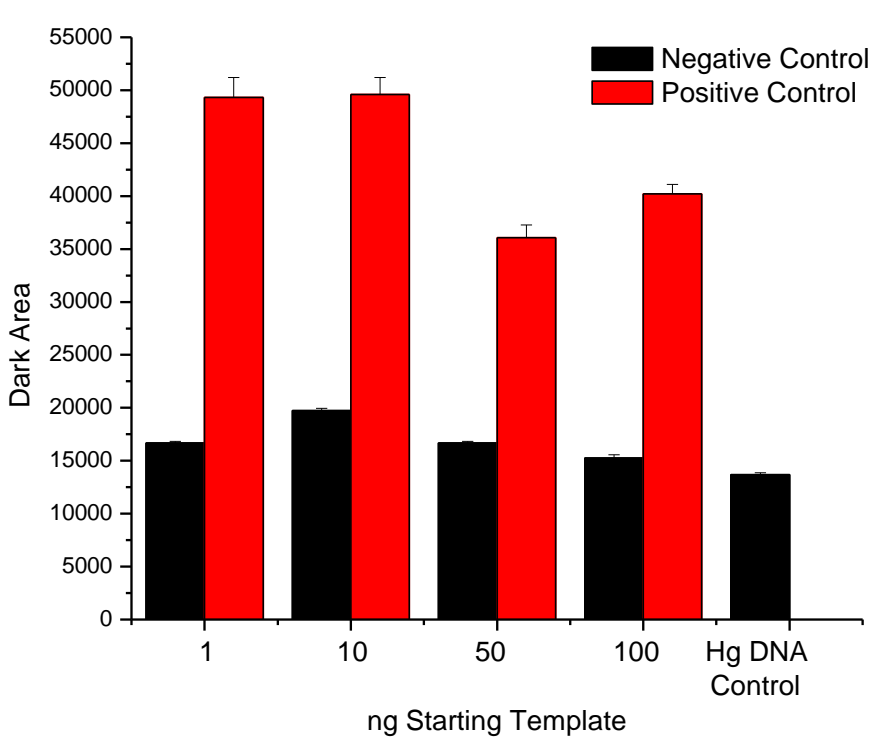

Figure 4: Analyzed samples on PiBA setup after running through Millipore column $(\mathrm{n}=3)$.

correlated to previous results. This supported the predicted hypothesis that the leftover template DNA could be competing with the inhibition of aggregation from the LAMP amplicons. The inhibition response at higher concentrations was recoverable upon filtration of the sample to remove any remaining starting template with a Milipore column (Fig. 4); thus this experiment confirming the predicted hypothesis. Unlike other bead detection methods which will still detect the result if the sample saturates the system, PiBA will provide false negatives even if a sample is present and has amplified. Therefore, samples of unknown sample concentration can mask the inhibition at varying rates which makes it difficult to accurately identify samples of interest.

Millipore columns were explored for correcting the 'masking effect' which can negatively impact the quantitative capability of PiBA. In working conditions, a linear increase in dark area is observed over time on the PiBA platform, which is indicative of the production of LAMP amplicons with increased amplification time (Fig. 5A). When amplified samples were run through a Millipore column to eliminate leftover starting template, it was soon discovered that the 
A

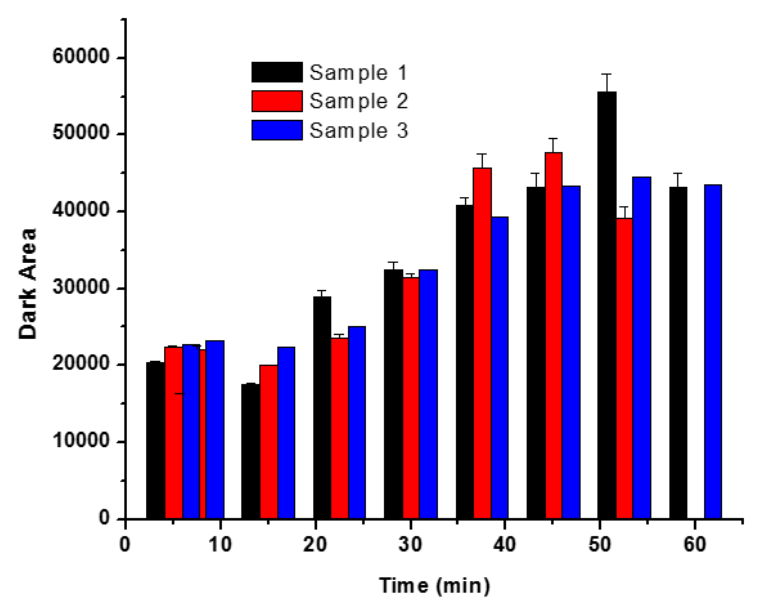

B

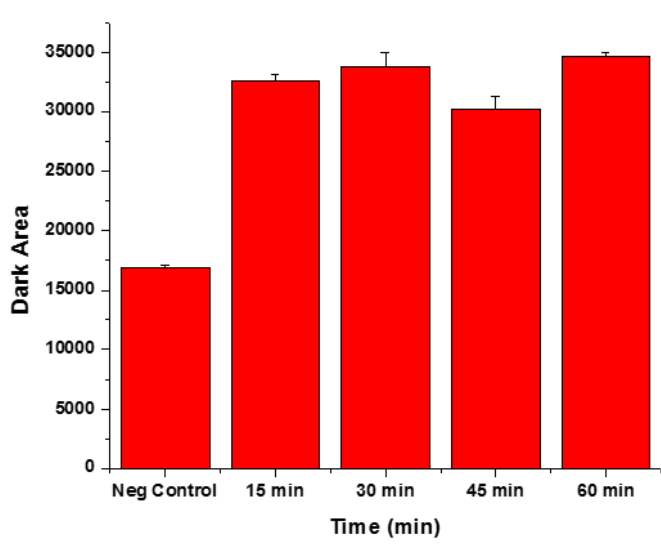

Figure 5: Quantitative Analysis of LAMP DNA (n=3). (A) Real-time analysis of triplicate LAMP samples on the PiBA platform. (B) Real-time analysis of amplified samples (starting template of $100 \mathrm{ng}$ ) after a Millipore column cleanup.

quantitative detection ability was no longer possible (Fig. 5B). This may be due to sample loss

during PCR cleanup. Since Millipore columns were not feasible, an alternative detection method was explored for possible quantitative detection of LAMP amplicons.

\subsubsection{Biotin Labeling during LAMP for HAA}

The LAMP mechanism uses four to six specific primers (F3, FIP, BIP, and B3 (LF and LB optional)) to identify sequence-specific regions alongside a target sequence. If any of the primer sequences do not match the template sequence perfectly, LAMP amplification of the target sequence will not occur ${ }^{1}$. During the amplification stage of LAMP, the FIP and BIP primers are those that play a primary role, with the LF and LB primers accelerating the rate of amplification. If either the FIP or BIP primers were modified with a tag, then it could transfer the tag only to the LAMP amplicons for detection of a given product. Several tags, including biotin and fluorescein isothiocyanate (FITC), have been previously used to tag LAMP amplicons without interfering with 
chaLAMP amplification ${ }^{20}$. As fluorescence requires complex and expensive instrumentation, we explored the use of biotin to assist with post-amplification bead aggregation.

The high affinity between biotin and streptavidin is well understood ${ }^{21-23}$ and has been previously exploited in many applications for the detection of target analytes ${ }^{24-28}$. To trigger bead aggregation with biotin, it was critical to enable biotin labeling in multiple sites of LAMP amplicons to enable the tethering of beads together. There were two ways to biotinylate amplified nucleic material in this manner: (i) directly biotinylate the starting template using a photoreactive biotinylation reagent or (ii) using custom made biotinylated primers to transfer biotin to amplicons during amplification. Biotinylating the starting template prior to amplification was not ideal as photoreactivation is time consuming and expensive. Furthermore, any leftover template DNA or RNA could interfere with the detection of LAMP amplicons as all nucleic material would be biotinylated. Therefore, the FIP and BIP primers were designed with a 5' end biotin modification to enable biotin labeling of amplicons (Fig. 6A).

\subsubsection{Data Analysis}

To determine whether biotin labeled LAMP products could trigger bead aggregation with streptavidin beads, a previously optimized Rift Valley Fever (RVFV) virus LAMP assay ${ }^{7}$ was explored. All primer sequences remained the same with exception of a 5' biotin modification on the FIP and BIP primers. All amplifications with RVFV cDNA $(n=3)$ provided visual turbid results at optimized amplification conditions, which indicated that primer biotin modification did not inhibit the amplification process. When placed on the agitation platform, we found that those samples containing the targeted sample of interest were the only ones to successfully induce bead aggregation (Fig. 6B). The lack of aggregation from biotinylated primers in the negative controls 
was attributed to the primers only having a biotin label on the 5 ' end, which by itself would not allow the tethering of beads together to trigger bead aggregation. Even with a 10-fold increase in the primer concentration in the negative control samples, the degree of aggregation (measured by dark area) was easily distinguishable from a positive control sample ( $1 \mathrm{ng}$ starting template) at $1 \mathrm{x}$ primer concentrations (Fig. 7). This is important, as a 10x primer concentration likely causes the formation of primer complexes that could create nonspecific bead aggregation. This concentration, however, is too high and causes LAMP amplification to fail.

The Mathematica algorithm, originally developed to discriminate the differences in bead aggregation between silica-coated magnetic particles and $\mathrm{DNA}^{14}$, could not always reliably differentiate dispersed streptavidin beads from those assays containing small amounts of
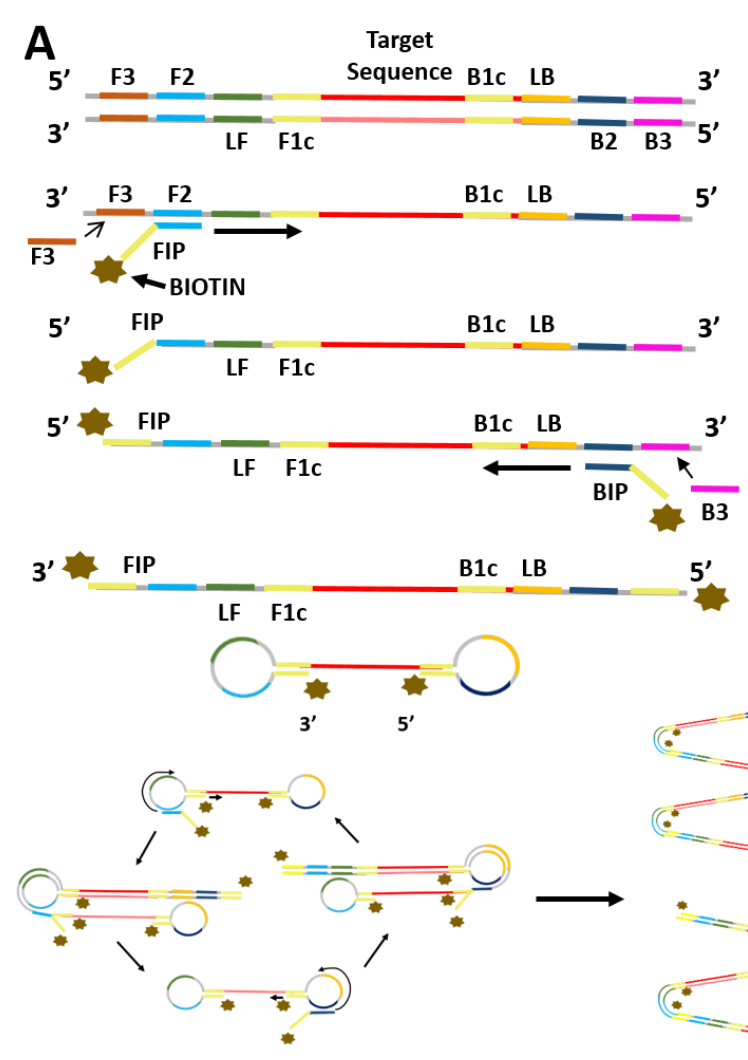

B
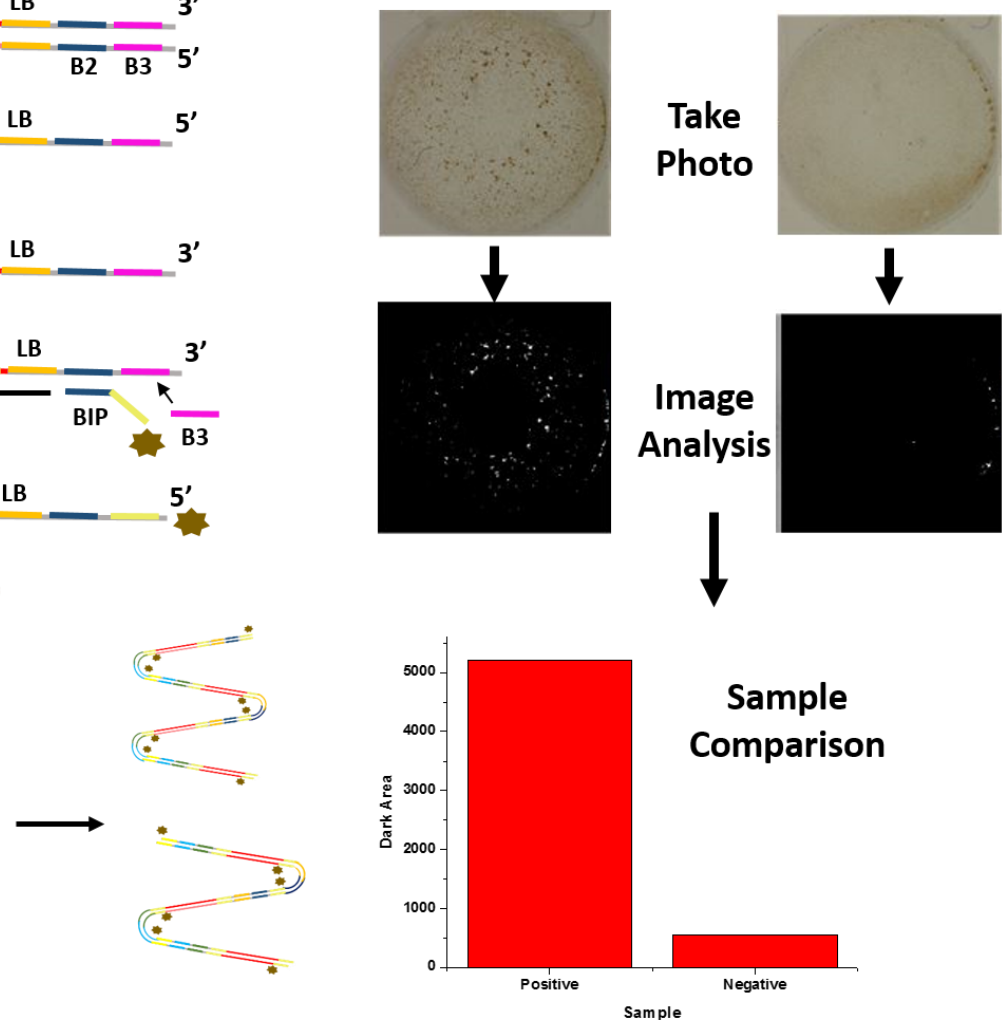

Figure 6: Schematic of LAMP amplification and biotin-streptavidin binding. (A) LAMP detailed mechanism with biotin-labelling. (B) Exemplary positive and negative LAMP controls with HAA platform using biotin-labeling. 
aggregation. This was likely due to

the lighter brown color of the

streptavidin particles that blended

with the background color of the

well. Therefore, the algorithm was

modified such that the image analysis

only looked at the saturation of the

image, and out of the scale of 0 to

255 , set a minimum threshold of 150 .

Instead of measuring the dispersion

of the beads, the dark area now

measured the amount of aggregation

present in the well. Increased

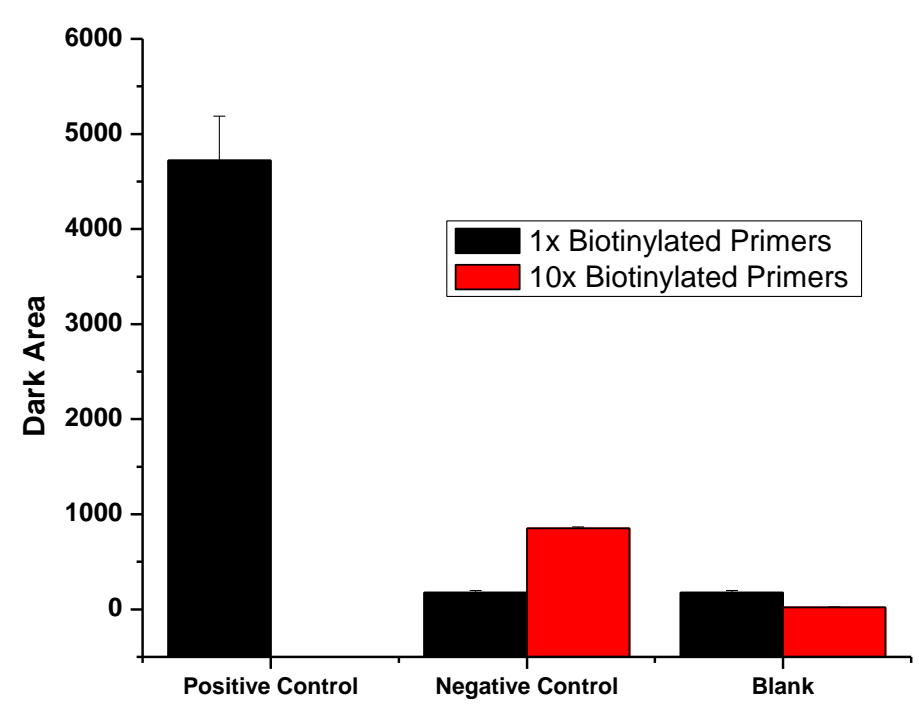

Figure 7: Biotinylation Primer Concentration Study. The contributing aggregation for HAA was compared for primer concentrations of $1 \mathrm{x}$ concentration (manufacturer instructions) and 10x in Tris-EDTA (TE) buffer in triplicates. The results are compared in dark area which is attributing to the amount of aggregation. Increased dark area corresponds to increasing bead aggregation.

aggregation, therefore, correlated to increasing dark area. Using this modified algorithm, samples containing a target analyte could now be discriminated by negative control samples, as shown in Figure 6B. All positive samples, therefore, could be identified by a dark area > 1000, and negative, or blank samples with readouts $<1000$.

\subsubsection{Characterizing Bead Aggregation}

Although preliminary results for HAA was shown to be effective, several parameters affecting the biotin-streptavidin bead aggregation were optimized: bead agitation speed, the interaction time between beads and biotin-tagged amplicons, streptavidin bead concentration, and the sample volume. 


\subsubsection{Bead Agitation}

Continuous agitation of the streptavidin beads prevents the beads from settling in the microwell and is an important factor in bead-assisted mixing ${ }^{16}$. However, the agitation speed needs to be optimized to avoid the creation of false positives through bead settling or false negatives by the disruption and prevention of bead aggregates. A vortexing speed of 130 RPM was initially explored, as this is the optimal setting for an oligonucleotide-bound streptavidin bead detection method $^{12,15}$. As shown in Figure 8A, an agitation speed of 130 RPM provided sufficient differentiation between positive and negative control samples, however, negative control samples were rarely dispersed due to bead settling. This led to an increased amount of false positives. At an increased vortexing speed of 550 RPM (commonly used for PiBA), the streptavidin beads were very well distributed in all negative controls, but the bead aggregates in positive samples were difficult to see and often interpreted as false negatives. Further exploration found that agitation speeds between 490 and 500 RPM were optimal for HAA. Due to increased reproducibility at 490 RPM, this value was chosen for all future experiments.

\subsubsection{Mixing Time}

The streptavidin-biotin interactions is one of the most stable in nature with an exceptionally high affinity $\left(\mathrm{K}_{\mathrm{a}} \sim 2.5 \times 10^{13} \mathrm{M}^{-1} \text { at } \mathrm{pH} 7.4 \text { and } 25^{\circ} \mathrm{C}\right)^{22}$. As the binding of streptavidin and biotin is biphasic, with multiple binding sites on each streptavidin bead, we would expect increased aggregation with increased mixing time. Therefore it is necessary to determine the maximum mixing time in the microwells with minimal evaporation. 
Initial mixing times of 5, 7, 10, and 12 minutes were chosen based on other bead detection mixing methods ${ }^{7,12,14-16}$ (see Fig. 8B). Starting template DNA (1 ng and $100 \mathrm{ng}$ ) served as low and high range of detection for LAMP amplifications to observe the sample mixing. After 5 minutes of mixing, we observed large discriminations between positive and negative samples, but a large variability between sample runs at $100 \mathrm{ng}$. Increasing the mixing time to 7-10 minutes increased the sample reproducibility without compromising the discrimination between a positive and negative sample. Once the mixing time exceeded 10 minutes, the beads started pushing towards the outside of the well, which was indicative of evaporation. To allow for maximum aggregation, a mixing time of 10 minutes was chosen.

\subsubsection{Bead Concentration}

Optimal bead concentration is important to avoid a loss in sensitivity due to oversaturation or false aggregation due to the image algorithm. The preliminary results of HAA showed promising discrimination of positive and negative control samples using a $10 \mathrm{mg} / \mathrm{mL}$ bead sample. However, following the modification in the image algorithm, fully dispersed beads at $10 \mathrm{mg} / \mathrm{mL}$ were misinterpreted as bead aggregates. To minimize this effect, the concentration of the beads was incrementally decreased $1 \mathrm{mg} / \mathrm{mL}$. Only when the bead solution was decreased 2 -fold in concentration would all false aggregation be fully eliminated (data not shown).

Three different bead concentrations were explored to find the optimal detection of target amplicons using 10 minutes of mixing: 4, 4.5, and $5 \mathrm{mg} / \mathrm{mL}$ (Fig. 8C). Just like in the mixing time experiments, each of these parameters were tested with $1 \mathrm{ng}$ and $100 \mathrm{ng}$ of starting template. At $4 \mathrm{mg} / \mathrm{mL}$, the samples containing the target DNA saturated the beads, providing similar dark area values for both $1 \mathrm{ng}$ and $100 \mathrm{ng}$ samples. This is indicative of a loss in sensitivity because the 
amount of starting template is linearly related to the amount of product generated ${ }^{1}$. Therefore, more biotinylated product would be expected in the $100 \mathrm{ng}$ sample than a $1 \mathrm{ng}$ sample. Increasing the bead concentration to $4.5-5 \mathrm{mg} / \mathrm{mL}$ yielded a distinguishable difference in aggregation between a $1 \mathrm{ng}$ and $100 \mathrm{ng}$ sample and a positive: negative dark area discrimination ratio > 20 . Due to increased discrimination between positive and negative control samples, a $5 \mathrm{mg} / \mathrm{mL}$ bead concentration was chosen for all future assays.
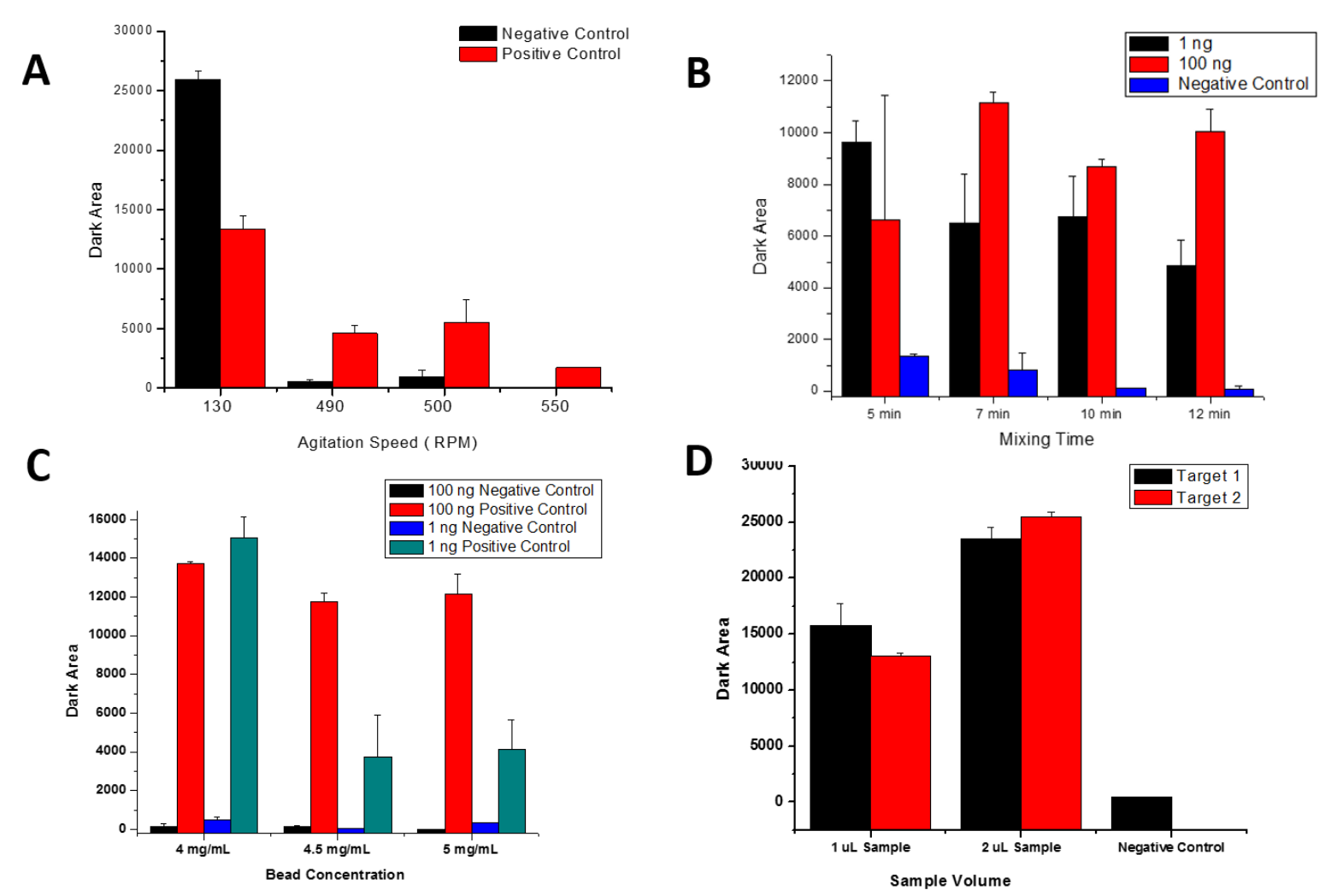

Figure 8: Optimization parameters for HAA assay $(n=3)$. (A) Comparison of the discrimination of positive $(1 \mathrm{ng} / \mu \mathrm{L})$ and negative control samples (just TE buffer) at varying agitation speeds. (B) Graph comparing magnetic mixing times for optimal detection. (C) Comparison of streptavidin bead concentration at an optimal 10 minute mixing time. (D) Comparing the volume of sample with two different target ampllifications at a 10 minute mixing and $5 \mathrm{mg} / \mathrm{mL}$ bead solution. 


\subsubsection{Sample Concentration}

Most bead detection platforms all require 1-2 $\mu \mathrm{L}$ of sample in a microwell holding $20 \mu \mathrm{L}^{7}$, 14, 16 which provide a sufficient discrimination between samples with the target and ones without any targets. To test whether it also applied to HAA, $1 \mu \mathrm{L}$ and $2 \mu \mathrm{L}$ sample aliquots from two different targeted amplification reactions were compared at all previously described optimization parameters. Target 1 was RVFV whereas target 2 was Salmonella enterica, both with $1 \mathrm{ng}$ of starting template. Sample aliquots of $1 \mu \mathrm{L}$ provided a large distinction between a positive control and negative control sample, as shown in Figure 8D. When the sample input was increased to 2 $\mu \mathrm{L}$, the differentiation between positive samples and negative sample increased, as expected with an increased amount of biotinylated product. As a $1 \mu \mathrm{L}$ aliquot provides satisfactory distinction between a positive and negative control sample, there was no need to use more than $1 \mu \mathrm{L}$ sample for future experiments.

\subsubsection{HAA Applications}

HAA has broad applicability, as it can be applied to any target with any amplification, so long as modified primers with biotin do not affect the amplification. For general amplification via PCR, one could use a forward and reverse $5^{\prime}$ biotinylated primer set to generate double stranded products with biotin labeled on both ends. For the purposes of this paper, previously optimized LAMP assays were used to show HAA applications for RVFV RNA for bio warfare agent detection, Salmonella enterica DNA for food pathogen detection, and allele specific drug genotyping with the P450 CYP2C19 gene (only *2 mutation and wild-type chosen). To adapt all optimized primer sets for HAA detection, the 5' ends of all FIP and BIP primers were modified with biotin, as shown in Table 1 (appendix). The LAMP reactions then proceeded as reported in 
literature ${ }^{7,10}$ to yield biotinylated products. It should be noted that the drug genotyping assay was amplified using the manufacturer directions instead of the altered reaction mix described ${ }^{10}$. All amplified targets provided significant bead aggregation, as shown in Figure 9A, and could be distinguished by image analysis from a negative control that contains only LAMP reagents without nucleic material (see Fig. 9B). Although the extraction of DNA from whole blood samples is not necessary, as LAMP is not as sensitive to PCR inhibitors such as heme, the leftover proteins within blood induced false aggregation of the streptavidin beads (data not shown).

A

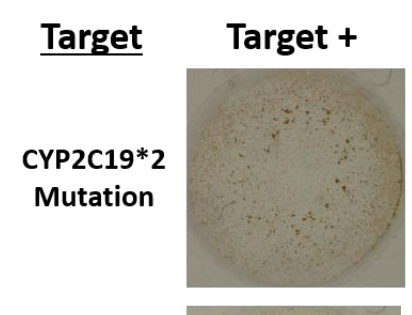

CYP2C19*2 Wild-type
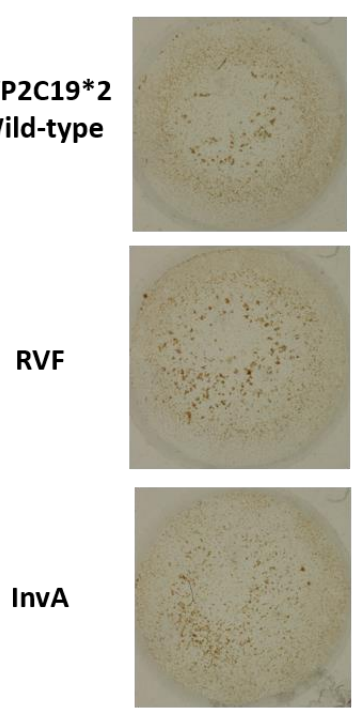

B

\section{Target -}
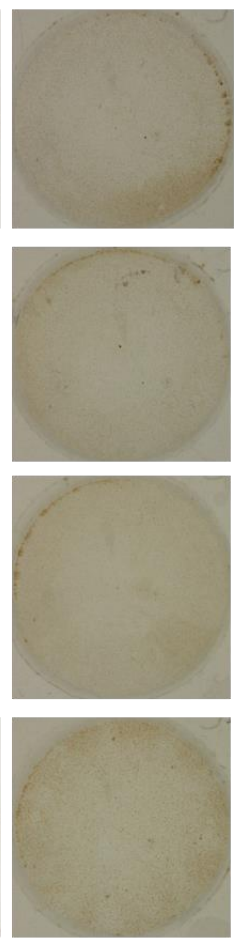

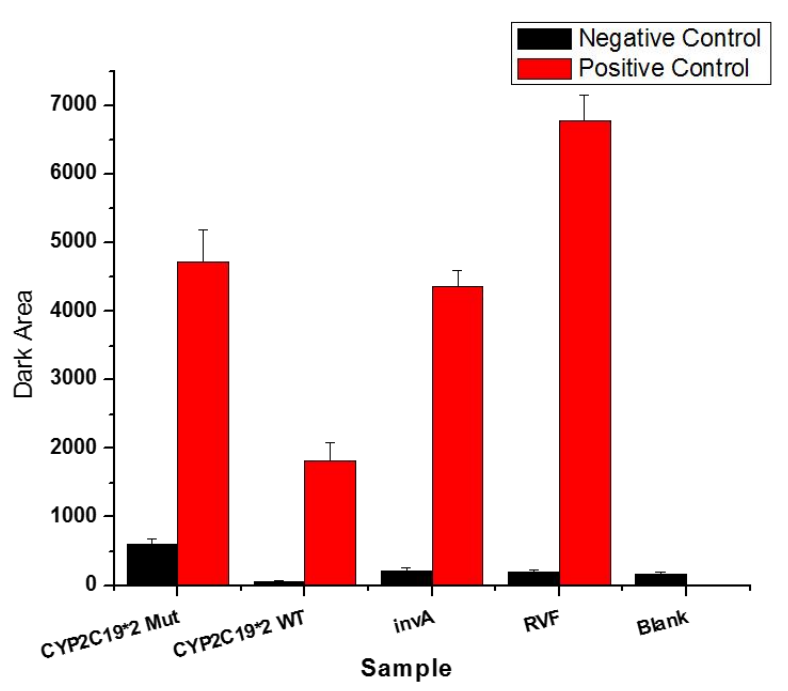

Figure 9: Comparisons of HAA assay with various LAMP targets. (A) Visual comparison of HAA assays with and without the target LAMP product. (B) Image analysis of target photos compared to a blank sample 


\subsubsection{Exploration of HAA Limitations}

Since the bead aggregation is

induced upon the production of biotinylated

LAMP amplicons, the limitations of HAA

is solely based on the limitations of the

amplification method. Manufacturer

instructions define $100 \mathrm{ng}$ as the maximum

DNA starting template for the LAMP

amplification kit however no low limit was

mentioned. As this needed to be defined, starting template concentrations of: $16 \mathrm{pg}$, 400 pg, 2 ng, 10 ng, 50 ng, and 100 ng

were amplified. As shown in Figure 10,

HAA could positively detect between 400

pg and 100 ng. Samples containing

between 16 - 400 pg were separately

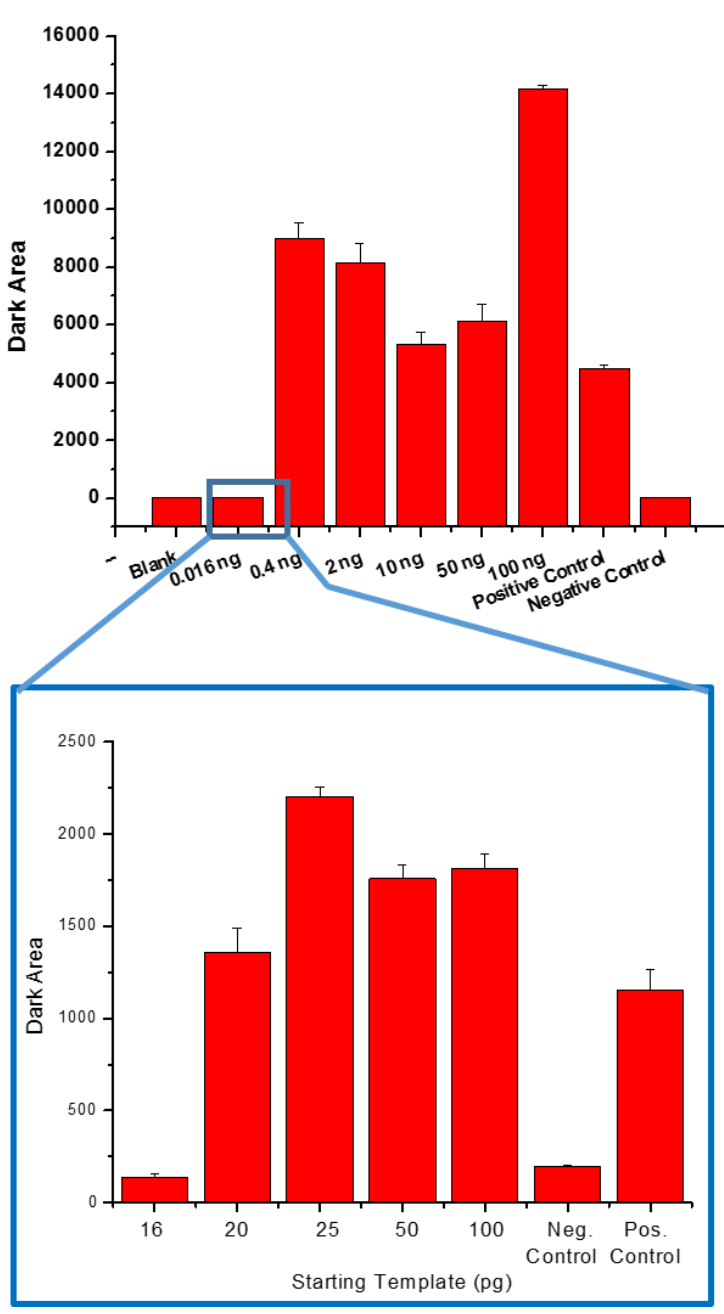

Figure 10: 60 minute HAA amplifications tested over a broad range of starting templates.

amplified for 60 minutes to see the lower limit of the LAMP reaction and compared to a separate positive control. As little as $25 \mathrm{pg}$ starting template (equivalent to $\sim 5$ copies of DNA) was successfully amplified as confirmed by turbidity detected by the naked eye. These results were verified on the HAA system, with positive amplification down to $20 \mathrm{pg}$ of starting template within a 60 minute amplification (Fig. 10). Although normalization of the dark area is necessary due to variability from run-to-run, HAA detection provides comparable detection limits to that 
A

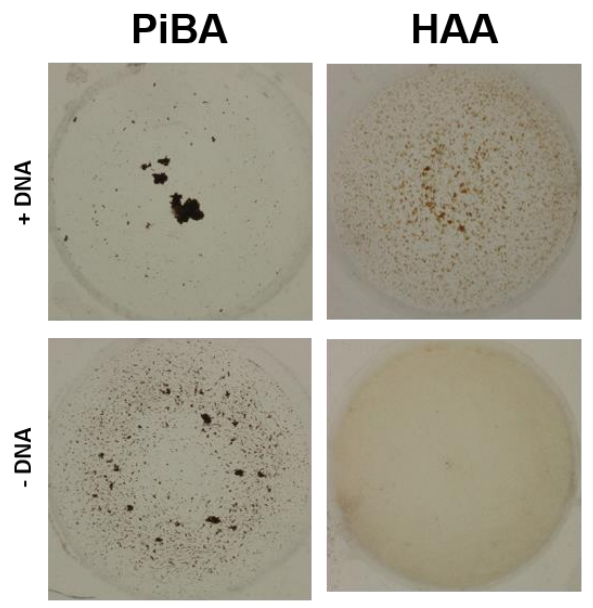

B

\begin{tabular}{|c|c|c|}
\hline Method: & PiBA & HAA \\
\hline Detection Ability & Quantitative & Quantitative \\
\hline $\begin{array}{c}\text { Sequence-Specific } \\
\text { Detection? }\end{array}$ & Yes & Yes \\
\hline $\begin{array}{c}\text { Detection Range } \\
\text { (ng/ } \mu \text { L) }\end{array}$ & $\sim 0.07->10$ & $0.02-100$ \\
\hline $\begin{array}{c}\text { Mechanism } \\
\text { LAMP Amplicon } \\
\text { Inhibition }\end{array}$ & $\begin{array}{c}\text { Biotin binding to } \\
\text { streptavidin }\end{array}$ \\
\hline
\end{tabular}

Figure 11: Comparison of PiBA and HAA Detection. (A) Snapshots of positive and negative control samples. (B) Table comparing qualities of PiBA and HAA

of $\mathrm{PiBA}^{7}$, but with improved detection bandwidth that spans over 4 orders of magnitude. Other comparisons between PiBA and HAA are shown in Figure 11.

The HAA platform was challenged with a real-time amplification sample to see whether the system could detect increased biotinylated product over time. To do this, a $20 \mathrm{ng}$ Salmonella enterica sample was amplified and analyzed over the course of a 60 minute amplification. The biotinylated product, as shown in Figure 12, is detected as early as 15 minutes. This detection capability shows potential of HAA as a quantitative method and the unique measurement capability, as a low limit of 20 ng is unprecedented of the detectable range of all bead detection methods to date.

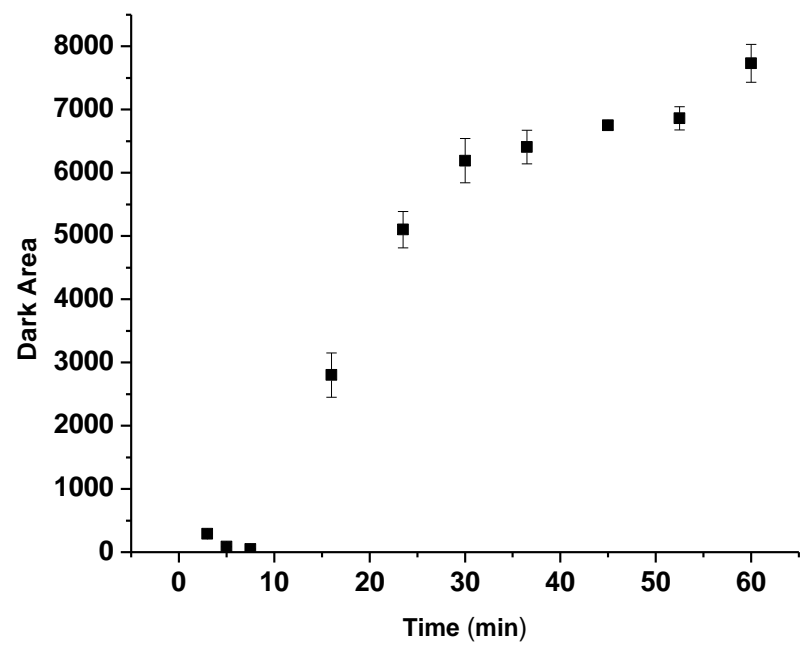

Figure 12: Real-time measure of amplification product during amplification with a $20 \mathrm{ng} \mathrm{RVFV}$ virus target $(\mathrm{n}=3)$. 


\subsection{Conclusions}

Here we define a new bead detection modality, HAA, for sequence-specific, sensitive, and accurate detection of target analytes. Although previously demonstrated as a useful bead detection method, PiBA could not accurately identify target analytes in human genomic samples containing more than 2 ng of DNA or RNA. Thus, HAA was proposed as an alternative bead detection method.

HAA can detect any DNA or RNA target, with detection limitations only defined by the restraints of the amplification method. Simple, rapid, and highly specific isothermal amplification was achieved with LAMP amplification. This method also allowed for a broad detection bandwidth between $20 \mathrm{pg}$ up to $100 \mathrm{ng}$. This provided HAA with a 10 -fold improvement over traditional PiBA with comparable sensitivity. More importantly is the broad applicability of HAA to rapidly detect even a single base mismatch for clinical genotyping or differentiate bacterial strains by optical analysis.

Like PiBA, HAA has the potential for quantitatively measuring the production of LAMP products in real time. Combined quantitative capability with single base discrimination opens the doors for countless applications. These include the potential for cost-effective and sensitive fluor-free quantitative PCR or personalized medicine.

\subsection{References}

1. N. Tomita, Y. Mori, H. Kanda and T. Notomi, Nat. Protocols, 2008, 3, 877-882.

2. T. Notomi, H. Okayama, H. Masubuchi, T. Yonekawa, K. Watanabe, N. Amino and T. Hase, Nucleic Acids Research, 2000, 28, e63.

3. K. Nagamine, T. Hase and T. Notomi, Molecular and Cellular Probes, 2002, 16, 223-229.

4. T. Iwamoto, T. Sonobe and K. Hayashi, Journal of clinical microbiology, 2003, 41, 2616-2622. 
5. M. Tsujimoto, K. Nakabayashi, K. Yoshidome, T. Kaneko, T. Iwase, F. Akiyama, Y. Kato, H. Tsuda, S. Ueda and K. Sato, Clinical Cancer Research, 2007, 13, 4807-4816.

6. Y. Mori and T. Notomi, Journal of infection and chemotherapy, 2009, 15, 62-69.

7. J. A. DuVall, J. C. Borba, N. Shafagati, D. Luzader, N. Shukla, J. Li, K. Kehn-Hall, M. M. Kendall, S. H. Feldman and J. P. Landers, Plos One, 2015, 10.

8. X. Zhao, Y. Li, L. Wang, L. You, Z. Xu, L. Li, X. He, Y. Liu, J. Wang and L. Yang, Molecular Biology Reports, 2010, 37, 2183-2188.

9. M. Safavieh, M. K. Kanakasabapathy, F. Tarlan, M. U. Ahmed, M. Zourob, W. Asghar and H. Shafiee, ACS Biomaterials Science \& Engineering, 2016, 2, 278-294.

10. M. Iwasaki, T. Yonekawa, K. Otsuka, W. Suzuki, K. Nagamine, T. Hase, K.-I. Tatsumi, T. Horigome, T. Notomi and H. Kanda, Genome Letters, 2003, 2, 119-126.

11. H. Aonuma, A. Badolo, K. Okado and H. Kanuka, in Nucleic Acid Detection: Methods and Protocols, eds. M. D. Kolpashchikov and V. Y. Gerasimova, Humana Press, Totowa, NJ, 2013, DOI: 10.1007/978-1-62703-535-4_10, pp. 121-127.

12. H. S. Sloane, K. A. Kelly and J. P. Landers, Analytical Chemistry, 2015, 87, 10275-10282.

13. J. Fischbach, N. C. Xander, M. Frohme and J. F. Glokler, Biotechniques, 2015, 58, 189-194.

14. D. C. Leslie, J. Li, B. C. Strachan, M. R. Begley, D. Finkler, L. A. L. Bazydlo, N. S. Barker, D. M. Haverstick, M. Utz and J. P. Landers, Journal of the American Chemical Society, 2012, 134, 56895696.

15. B. C. Strachan, H. S. Sloane, J. C. Lee, D. C. Leslie and J. P. Landers, Analyst, 2015, 140, 20082015.

16. D. A. Nelson, B. C. Strachan, H. S. Sloane, J. Li and J. P. Landers, Analytica Chimica Acta, 2014, 819, 34-41.

17. K. A. Melzak, C. S. Sherwood, R. F. B. Turner and C. A. Haynes, Journal of Colloid and Interface Science, 1996, 181, 635-644. 
18. H. Nogami, H. Tsutsumi, T. Komuro and R. Mukoyama, Forensic Science International-Genetics, 2008, 2, 349-353.

19. Y. Sun, Y. C. Kwok and N.-T. Nguyen, Journal of Micromechanics and Microengineering, 2006, 16, 1681-1688.

20. Y. L. Sun, C. H. Yen and C. F. Tu, Journal of Veterinary Medical Science, 2014, 76, 509-516.

21. M. Srisa-Art, E. C. Dyson, A. J. deMello and J. B. Edel, Analytical Chemistry, 2008, 80, 70637067.

22. L. Deng, E. N. Kitova and J. S. Klassen, Journal of The American Society for Mass Spectrometry, 2012, 24, 49-56.

23. P. Weber, D. Ohlendorf, J. Wendoloski and F. Salemme, Science, 1989, 243, 85-88.

24. R. Ahirwar, S. Tanwar, S. Parween, A. Kumar and P. Nahar, Analyst, 2014, 139, 2186-2192.

25. Z. Deng, Y. Ge, Q. Cao and K. Han, Bioorganic \& Medicinal Chemistry Letters, 2011, 21, 69056908.

26. Z. Yang, X. C. Sun, T. Wang, C. Lei, Y. Liu, Y. Zhou and J. Lei, Biomedical Microdevices, 2015, 17.

27. J. Lei, C. Lei, T. Wang, Z. Yang and Y. Zhou, Applied Physics Letters, 2013, 103.

28. T.-E. Du, Y. Wang, Y. Zhang, T. Zhang and X. Mao, Analytica Chimica Acta, 2015, 861, 69-73. 


\section{Chapter 2 Appendi}

Table 1: Primer sets used for testing HAA assay

\begin{tabular}{|c|c|c|}
\hline Target & Primers & Sequence $\left(5^{\prime}-3^{\prime}\right)$ \\
\hline \multirow[t]{6}{*}{ invA } & FIP & [Biotin-5]GCGCGGCATCCGCATCAATATITTTGCCCGGTAAACAGATGAGT \\
\hline & BIP & [Biotin-5]GCGAACGGCGAAGCGTACTGTITTCGCACCGTCAAAGGAAC \\
\hline & F3 & CGGCCCGATTTTTCTCTGG \\
\hline & B3 & CGGCAATAGCGTCACCTT \\
\hline & $\mathrm{LF}$ & GGCCTTCAAATCGGCATCAAT \\
\hline & LB & GAAAGGGAAGCCAGCTTTACG \\
\hline \multirow[t]{6}{*}{ RVFV } & FIP & [Biotin-5]AGCACCTCTGGATTCTCATTTATTIITTCAGAAATTGAGAGACCGTTT \\
\hline & BIP & [Biotin-5]AGAACAGGCCCAGAAATATTGTTTTTGACAATGATGACACAACAC \\
\hline & F3 & TGGGGATCTAGGAAGAAGTT \\
\hline & B3 & GAGGCCATGACTTTACAAACT \\
\hline & $\mathrm{LF}$ & GTTCAATCCAGTTCTCTGGTATGTT \\
\hline & LB & CATTGCAGAGAAAGTCCATAGCC \\
\hline \multirow[t]{2}{*}{$\begin{array}{l}\text { CYP2C19*2 } \\
\text { Wild-type }\end{array}$} & FIP & [Biotin-5]CCGGGAAATAATCTTTTAATTTAATAAATTATTGTTTTCTCTTAG \\
\hline & BIP & [Biotin-5]CGGGAACCCGTGTTCTTTTACTTTCTCC \\
\hline \multirow[t]{2}{*}{$\begin{array}{l}\text { CYP2C19*2 } \\
\text { Mutation }\end{array}$} & FIP & [Biotin-5]CTGGGAAATAATCTTTTAATTTAATAAATTATTGTITTCTCTTAG \\
\hline & BIP & [Biotin-5]CAGGAACCCGTGTTCTTTTACTTTCTCC \\
\hline \multirow[t]{4}{*}{$\begin{array}{c}\text { CYP2C19*2 } \\
\text { Common } \\
\text { Primers }\end{array}$} & F3 & CCAGAGCTTGGCATATTGTATC \\
\hline & B3 & AGGGTTGTTGATGTCCAT \\
\hline & $\mathrm{LF}$ & GATAGTGGGAAAATTATTGC \\
\hline & LB & CAAATTACTTAAAAACCTTGCTT \\
\hline
\end{tabular}




\section{Development of a Novel Loop-mediated Isothermal Amplification Panel for Body Fluid Identification}

\section{Summary}

Messenger RNA profiling for body fluid identification (BFI) is a useful approach to collect contextual information surrounding a crime. Current methods require expensive fluorescent probes or time-consuming sample preparation. To simplify this process, we report the development of an inexpensive, fluorescence-free detection method that combines a universal operating procedure with a high-throughout (96-well plate) platform for simultaneous detection of mRNA markers from blood, cell-free semen, sperm, saliva, and vaginal fluid. Full BFI analysis of 23 samples was completed in under 3 hours using smart phone optical detection and analysis and show efficacy of the method in a validated blind study. The results provide an efficient and accurate method that can supplement the current biochemical tests in a forensic laboratory.

\subsection{Introduction}

Genetic DNA profiling via short tandem repeat (STR) analysis is heavily relied on for unique identification of an individual in a criminal case. Body fluid identification (BFI) has gained recent attention due to important contextual information that is not provided through STR analysis. The identification of saliva versus semen on a victim's clothing, for instance, will significantly change the focus of an investigation. To be effective with the current DNA workflow, BFI methods should be non-destructive, sensitive and accurate, and with high specificity $^{1}$.

Traditionally, forensic labs look to presumptive tests followed by confirmatory testing for the identification of blood, saliva, and semen. These presumptive tests interact with the body 
fluids to produce a colorimetric change, visible to the eye. Although these tests are sensitive and provide rapid results, they are not human specific and are susceptible to false positives ${ }^{2}$. With increased specificity, confirmatory tests can provide a colorimetric answer via antibody-antigen interactions with increased confidence ${ }^{3}$. However, even the most popular immunological test suffer from false positives so there still remains a need for improved BFI methods ${ }^{2,4-7}$.

Messenger RNA (mRNA) profiling is a promising method for identification of biological material due to mRNA stability and DNA workflow compatibility via co-purification of RNA with $\mathrm{DNA}^{8,9}$. Forensically-relevant body fluids such as vaginal fluid and menstrual blood could not be previously be identified via catalytic or enzymatic methods but are now ascertained via mRNA markers such as MUC4 and MMP7, respectively ${ }^{2,10}$. Although multiplexed mRNA profiling assays have been reported by methods including quantitative real-time polymerase chain reaction (qRT-PCR) assays or high resolution melt (HRM) analysis, these methods use expensive instrumentation and may provide false negatives or false positive identification ${ }^{10-12}$.

A more recent Nanostring ${ }^{\circledR}$ barcoding system uses 18-23 different mRNA targets and probe hybridization to identify fluids such as blood, semen, saliva, and vaginal fluid, and sweat ${ }^{10,}$ 13. Accurate identification of blood and semen were possible using mRNA markers, however, due to the use of multiple non-specific mRNA markers, a statistical algorithm had to be used for determining the 'probability' of a stain containing either saliva, vaginal fluid, or sweat. In addition, this method required large sample volumes (50 $\mu \mathrm{L}$ stains) in combination with a lengthy $12-24$ hour hybridization time frame per sample before body fluids could be identified $^{13}$.

An alternative and simple BFI method was proposed for rapid testing of blood using a real-time detection via reverse-transcription loop mediated isothermal amplification (RT- 
LAMP $)^{14}$. The high specificity, rapid isothermal amplification, and single-tube approach of LAMP detection via turbidity measurements eliminated the false identification encountered with RT-qPCR and simplified the sample processing. This method can accommodate trace samples at $10^{-5}$ ng of RNA but only applies to blood samples and has yet to be demonstrated with any other body fluid.

Here, we describe the development of a body fluid panel for the identification of blood, saliva, vaginal fluid, semen, and azoospermatic semen samples using RT-LAMP coupled with a simple optical detection method. A single fluorescent-free operating procedure was optimized for the simultaneous identification of all of the aforementioned body fluids in a simpler manner. The addition of a metal-indicator dye, hydroxylnathphol blue (HNB), provides a visual colorimetric readout of LAMP reactions that can be captured by a smart phone. Placement on a 96-well format increases the sample efficiency to provide for a sample-to-answer time of $\sim 1.5$ hours for up to 23 samples simultaneously.

\subsection{Materials and Methods}

\subsubsection{Sample Collection}

Whole blood samples, provided by the University of Virginia Medical School, were collected via a standard venipuncture technique as a part of routine care and treated with $5.4 \mathrm{mg}$ of $\mathrm{K}_{2}$ EDTA for anti-coagulation. These samples were collected biweekly and stored at $4-8{ }^{\circ} \mathrm{C}$ until used. All other de-identified buccal swabs, vaginal swabs, and semen samples were collected using procedures approved by the University Institutional Review Board (IRB). Freshly ejaculated semen samples were aliquoted into $50 \mu \mathrm{L}$ volumes and stored at $4{ }^{\circ} \mathrm{C}$ until needed. Fresh deidentified vaginal and buccal swabs were dried and stored in a dark drawer at room temperature. 
To prepare dry stained samples, the body fluids of interest were manually spotted on 2" x 2" squares of blue denim, dark denim, a dark blue sheet, or cotton material and kept in a dark drawer overnight at room temperature. Stained sample volumes included $10 \mu \mathrm{L}$ blood or semen, or wiping a fresh saliva or vaginal swab on the material for 30 seconds.

Azoospermatic sperm samples were processed from collected ejaculated semen samples. Approximately $50 \mu \mathrm{L}$ aliquots were placed in a $0.2 \mathrm{~mL}$ PCR tube and centrifuged at maximum speed $(13,400$ revolutions per minute) for 10 minutes. The supernatant was transferred to a new $0.2 \mathrm{~mL}$ PCR tube. This process was repeated for another 5 minutes and the supernatant again placed in a clean $0.2 \mathrm{~mL}$ tube. Aliquots from each sample was stained with SYTO 11® (Life Technologies; Carlsbad, CA) and examined under a fluorescent microscope (Zeiss Axio Scope.A1; Carl Zeiss Microscopy Ltd.; Jena, Germany) to ensure that there were no sperm cells prior to further sample processing. All samples were stored at $4{ }^{\circ} \mathrm{C}$ until needed.

\subsubsection{RNA Isolation}

A Qiagen RNeasy Mini kit (Valencia, CA) was used for all purifications of samples using manufacturer instructions. To lyse blood, vaginal swab, buccal swab, or semen samples, $350 \mu \mathrm{L}$ of RLT buffer was combined with $90 \mu \mathrm{L}$ RNA-free water, $10 \mu \mathrm{L}$ Proteinase K (Qiagen, Valencia, $\mathrm{CA}$ ), and $4.5 \mu \mathrm{L}$ of $\beta$-mercaptoethanol (Sigma Aldrich, St. Louis, MO). The samples were then incubated at $56{ }^{\circ} \mathrm{C}$ for 10 minutes. All swab samples were placed in a $0.5 \mathrm{~mL}$ tube that had been punctured with a 21 gauge needle in the bottom of the tube. These tubes were placed in a $1.5 \mathrm{~mL}$ microcentrifuge tube and centrifuged at a short spin cycle for 1-2 seconds at maximum speed ${ }^{15,16}$. The fractions spun through to the $1.5 \mathrm{~mL}$ tube were combined with the original lysed sample. The 
RNeasy kit manufacturer instructions were used for the remaining RNA purification steps. Once finished, all samples were stored at $-20^{\circ} \mathrm{C}$ until needed.

\subsubsection{Messenger RNA Marker Selection and LAMP Optimization}

We chose B-globin (HBB; accession no. NM000518.4) for blood identification, human beta-defensins (HBD-1; accession no. NM005218.3) for vaginal fluid, human semenogelin-1precursor (SEMG1; accession no. NM003007.4) as a semen marker, and histatin-3 precursor (HTN3; accession no. NM000200.2) for saliva detection. All LAMP primers were designed using Primer Explorer V4 and purchased from Eurofins MWG Operon (Huntsville, AL). A Loopamp DNA amplification kit (Eiken Chemical Co., Ttd, Tokyo, Japan) was used in combination with a reverse transcriptase (RT) kit (High Capacity RNA-to cDNA ${ }^{\mathrm{TM}}$ kit; Thermo Fisher Scientific, Waltham, MA) for individual body fluid LAMP optimizations and specificity testing according to the manufacturer instructions. Reaction volumes were reduced to $5 \mu \mathrm{L}$ and consisted of $1 \mathrm{x}$ reaction mix (40 mM Tis- $\mathrm{HCl}$ (pH 8.8), $20 \mathrm{mM}(\mathrm{NH} 4) 2 \mathrm{SO} 4,16 \mathrm{mM} \mathrm{MgSO} 4,20 \mathrm{mM} \mathrm{KCl}, 0.2 \%$ Tween, 1.6 M Betaine), 20 pmol LF and LB primers, 5 pmol for F3 and B3 primers, and 40 pmol for FIP and BIP, and $8 \mathrm{U}$ Bst polymerase. Approximately $0.5 \mu \mathrm{L}$ sample volumes were added to reaction volumes. A Biorad MyCycler Thermal Cycler (Bio-Rad Laboratories Inc., Hercules, CA) was used for all amplifications. Initial LAMP reactions were examined visually for increased turbidity and analyzed on Agilent 2100 instrumentation using DNA 1000 series II kits (Agilent Technologies, Santa Clara, CA) for confirmation of amplification.

Once individual LAMP reactions were optimized, all LAMP amplifications transitioned to a Loopamp RNA Amplification kit (Eiken Chemical Co., Ltd, Tokyo, Japan). Reaction volumes were increased to $10 \mu \mathrm{L}$ for optimized colorimetric detection of LAMP with all previously 
mentioned concentrations remaining the same. Approximately $1 \mu \mathrm{L}$ of sample was placed in each LAMP reaction.

\subsubsection{Colorimetric LAMP Analysis}

Hydroxynaphthyl blue (HNB) dye (Sigma Aldrich, St. Louis, MO) was added to each LAMP reaction to a final concentration of $120 \mu \mathrm{M}$ in an amplification volume of $10 \mu \mathrm{L}$. Samples were amplified in 96-well plates (cat \# 2239441; Bio-Rad Laboratories Inc., Hercules, CA) at $63{ }^{\circ} \mathrm{C}$ for up to a 60 minute amplification, with a 2 minute incubation at $95{ }^{\circ} \mathrm{C}$ to denature the $\mathrm{BST}$ polymerase. Once the amplification was finished, the 96-well plate was placed inside of an in-house built photo box made of poly methyl methacrylate (PMMA). Images of all sample wells were taken with an iPhone 6 (Apple Inc, Cupertino, CA) cell phone. The images were analyzed in ImageJ software using a hue, saturation, brightness (HSB) filter (HSB stack) surface plot to capture a hue profile for each well.

\subsection{Results}

\subsubsection{LAMP Optimization}

A unique mRNA marker for each body fluid was chosen based on fluid specificity and include HBB (blood), HBD-1 (vaginal fluid),

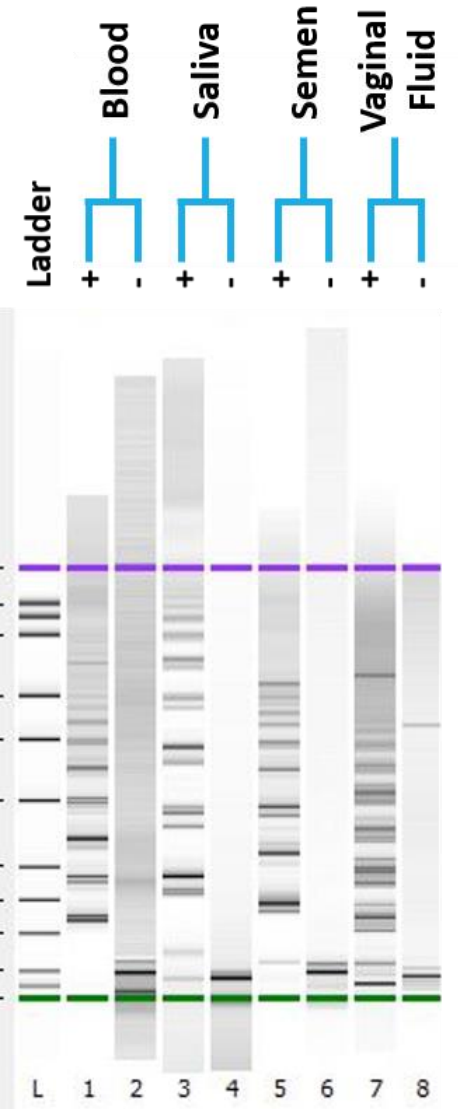

Figure 1: Electrophoretic gel displaying positive and negative LAMP controls of each body fluid using identified mRNA markers for blood, saliva, semen, and vaginal fluid. 
SEMG1 (semen), and HTN3 (saliva). Two of the five designed primer sets were chosen for each fluid in initial BFI testing. Extracted cDNA samples from each body fluid was used to test designed primers for successful amplification at temperatures between $60-65^{\circ} \mathrm{C}$. Each of these primer sets did amplify the targeted body fluid at one or more temperatures. Figure 1 shows an example electropherogram of the successful amplification of all targeted body fluids at $63{ }^{\circ} \mathrm{C}$ using one of the two selected primer sets.

Although the designated primers allowed successful amplification of targeted body fluids, the specificity of each assay needed to be analyzed. To do this, cDNA from each body fluid was tested against each primer set, with the inclusion of a negative control (containing only LAMP reagents with Tris-EDTA (TE) buffer) and a non-specific positive control (1 ng pre-purified human genomic DNA). Despite the high specificity that LAMP amplifications offer, three of the four primer sets from Figure 1 amplified non-specifically at $63^{\circ} \mathrm{C}$. An amplification map was created to find a set of primers that could selectively amplify each targeted body fluid over a $60-65{ }^{\circ} \mathrm{C}$ range (see Table 1; sequences are found in Table 1-4A (see appendix)). Using this map, we found three of the four body fluids were specific at both $63{ }^{\circ} \mathrm{C}$ and $65^{\circ}$, therefore, the number of

Table 1: Amplification Map of Explored Primer Sequences

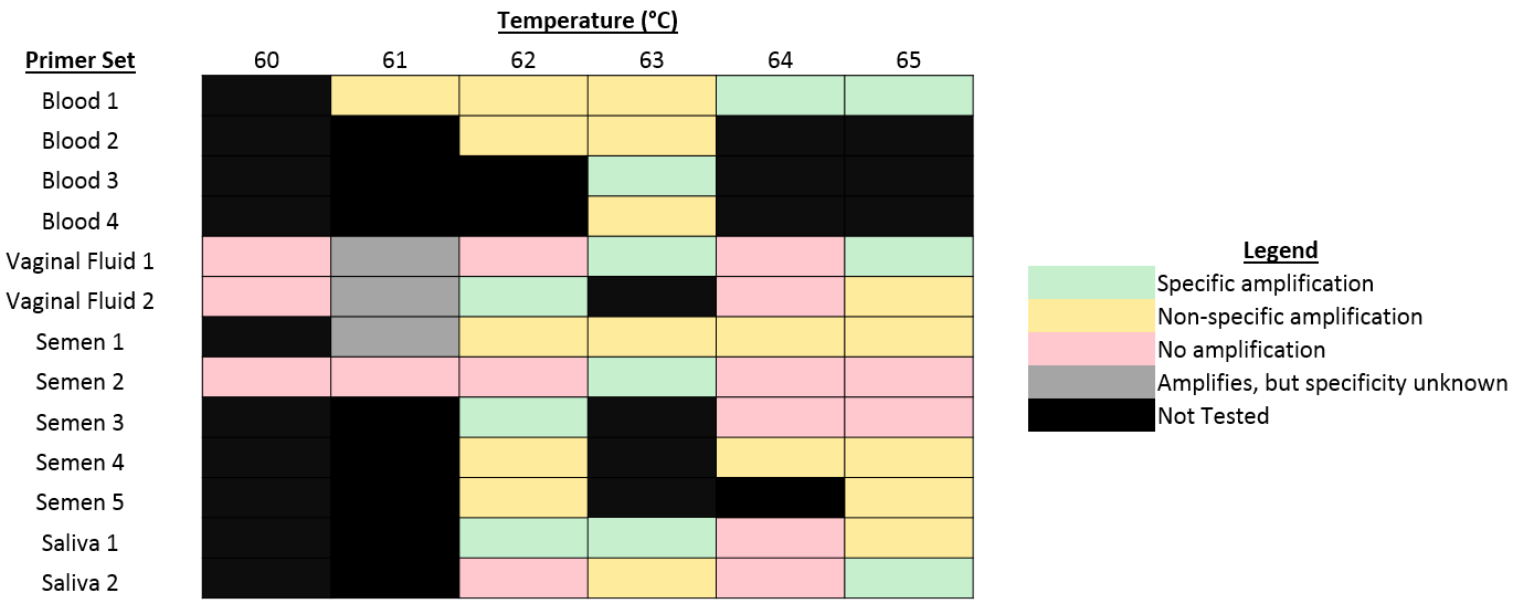



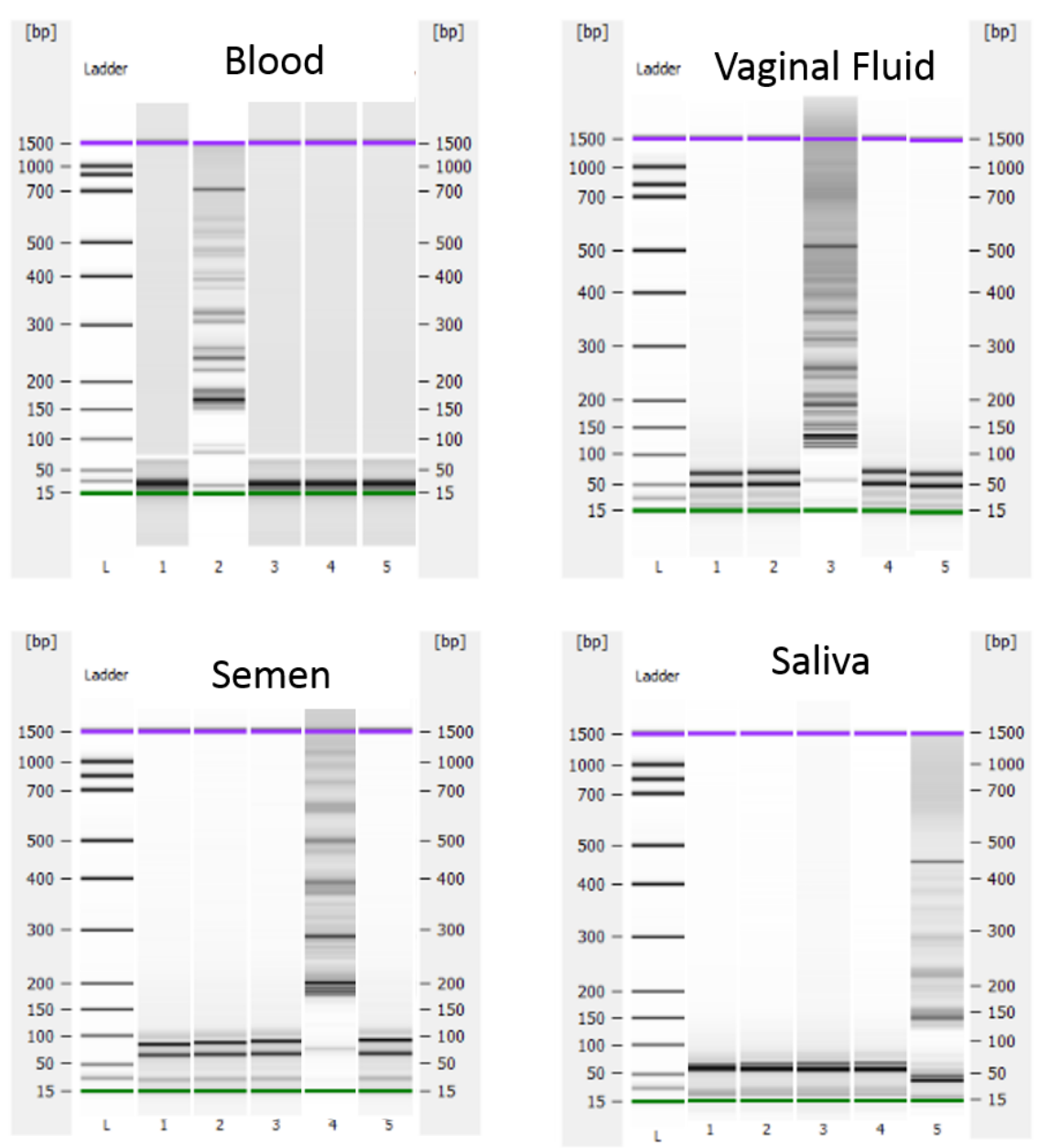

Figure 2: Electropherograms displaying specific amplification only to the targeted fluid of interest: Tris-EDTA buffer (Lane 1; negative control); blood (lane 2); vaginal fluid (lane 3); semen (lane 4); and saliva (lane 5).

primer sets were expanded for semen and blood. With this expansion of primer assays, we could achieve full specificity of all targeted body fluids at $63{ }^{\circ} \mathrm{C}(\mathrm{n}=20)$ as shown in Figure 2.

To shorten the sample-to-answer time for BFI identification, a RT-LAMP kit was used for direct amplification from RNA-extracted samples. This method has been used from RNA samples for bacteria and human-derived materials with rapid results ${ }^{14,17,18}$. Changing from a DNA LAMP kit to a RT-LAMP kit eliminated a 60 minute conversion of RNA to cDNA and did not negatively affect the efficiency of amplification, as observed visually via turbidity. Due to improved sample processing, the RT-LAMP kits were used for all future amplifications. 


\subsubsection{Universal Lysing Procedure}

To detect all targeted body fluids simultaneously, it was critical to develop a single universal sample preparation procedure for the lysis and extraction of all samples. Thus far, sample lysis were done according to different recipes previously described in the literature ${ }^{19}$. All recipes required different volumes of Qiagen RLT lysis buffer with 0, 25, or $39 \mathrm{mM}$ dithiothreitol (DTT) and the occasional use of Proteinase K (for vaginal swab and saliva samples). After extensive experimentation, we found that $350 \mu \mathrm{L}$ of Qiagen RLT buffer, $0.2 \mathrm{mg}$ proteinase $\mathrm{K}$, and $35 \mathrm{mM}$ DTT was optimal for the lysis of semen samples. When blood, semen, and vaginal swab samples were lysed with this recipe, all fluids reproducibly amplified within 30 minutes $(\mathrm{n}=10)$. However, the saliva LAMP assay weakly amplified at 60 minutes or failed to amplify at all $(\mathrm{n}=10)$. In a control study, we found that the amplification of saliva was hindered with increasing DTT concentrations (Table 2). As DTT is necessary for the lysis of sperm cells, it was important to find a minimum concentration to lyse the sperm cells without inhibiting the amplification of saliva samples. Table 2 shows that $32 \mathrm{mM}$ was the optimal concentration of DTT for semen and saliva samples, but only allowed saliva Table 2: Comparison of varying concentrations of DTT with the rate of amplification for semen and samples to amplify around 40 saliva samples

minutes.

Another reducing agent, 2-mercaptoethanol ( $\beta$-me), was explored as an alternative to DTT because of its known compatibility with Qiagen

\begin{tabular}{|c|c|c|}
\hline $\begin{array}{c}\text { Concentration } \\
\text { DTT }\end{array}$ & Semen RNA & Saliva RNA \\
\hline $39 \mathrm{mM}^{¥}$ & Amp $<30 \mathrm{~min}$ & No amp \\
\hline $37 \mathrm{mM}$ & Amp $<30 \mathrm{~min}$ & Amp $>45 \mathrm{~min}$ \\
\hline $35 \mathrm{mM}$ & $\mathrm{Amp}<30 \mathrm{~min}$ & $\mathrm{Amp}>45 \mathrm{~min}$ \\
\hline $32 \mathrm{mM}$ & $\mathrm{Amp}<30 \mathrm{~min}$ & $\mathrm{Amp} \sim 40 \mathrm{~min}$ \\
\hline $30 \mathrm{mM}$ & $\mathrm{Amp} \sim 40 \mathrm{~min}$ & $\mathrm{Amp} \sim 40 \mathrm{~min}$ \\
\hline $25 \mathrm{mM}$ & $\mathrm{Amp} \sim 45 \mathrm{~min}$ & $\mathrm{Amp}<30 \mathrm{~min}$ \\
\hline
\end{tabular}

$¥=$ Recommended DTT for sperm cells

$*=$ Recommended DTT for saliva 
Table 3: Comparison of DTT vs $\beta$-mercaptoethanol ( $\beta$-me) as a reducing agent for the LAMP Assay

\begin{tabular}{|c|c|c|c|c|c|c|c|c|c|c|}
\hline & \multicolumn{2}{|c|}{$35 \mathrm{mM}$ DTT } & \multicolumn{2}{c|}{$32 \mathrm{mM}$ DTT } & \multicolumn{2}{c|}{$0.8 \% \mathrm{v} / \mathrm{v} \beta-\mathrm{me}$} & \multicolumn{2}{c|}{$1 \% \mathrm{v} / \mathrm{v} \beta-\mathrm{me}$} & \multicolumn{2}{c|}{$1.3 \% \mathrm{v} / \mathrm{v} \beta-\mathrm{me}$} \\
\hline $\begin{array}{c}\text { Time } \\
(\mathrm{min})\end{array}$ & Semen & Saliva & Semen & Saliva & Semen & Saliva & Semen & Saliva & Semen & Saliva \\
\hline 25 & + & - & + & - & - & - & + & - & + & - \\
\hline 32 & + & - & + & - & - & - & + & + & + & + \\
\hline 40 & + & - & + & + & - & + & + & + & + & + \\
\hline
\end{tabular}

chemistry. Table` 3 shows a comparison of a series of sample lysis protocols containing optimal DTT concentrations (32 and $35 \mathrm{mM}$ ) and concentrations of $0.8 \%, 1 \%$ (manufacturer recommended), and $1.3 \%(\mathrm{v} / \mathrm{v}) \beta$-me $(\mathrm{n}=3)$. Overall, samples containing $\geq 1 \% \beta$-me provided more efficient amplification of saliva and semen samples than either concentration of DTT. Blood and vaginal fluid samples were tested in triplicates at $1 \% \beta$-me and also reproducibly amplified in less than 30 minutes. As $\beta$-me provided more efficient amplification for the targeted body fluids, it was integrated into the lysis for all future sample processing.

\subsubsection{Dried Sample Stains}

With a universal method established for all body fluids, the LAMP amplification method was challenged with difficult samples that cannot be easily tested by conventional chemical and enzymatic methods. These samples include dry fluid stains on denim and dark synthetic dye materials that can interfere with the visual interpretation of conventional colorimetric tests. Even though LAMP amplifications have been shown to be incredibly robust towards PCR inhibitors ${ }^{20}$, ${ }^{21}$, the extraction of RNA prior to LAMP amplification will likely eliminate the presence of any inhibitors. For proof-of-feasibility, $10 \mu \mathrm{L}$ of whole blood was spotted on $\sim 51 \mathrm{~mm}$ x $51 \mathrm{~mm}$ cuttings of the blue and black denim materials and dried overnight ( $\mathrm{n}=3$ each for 2 donors). From 

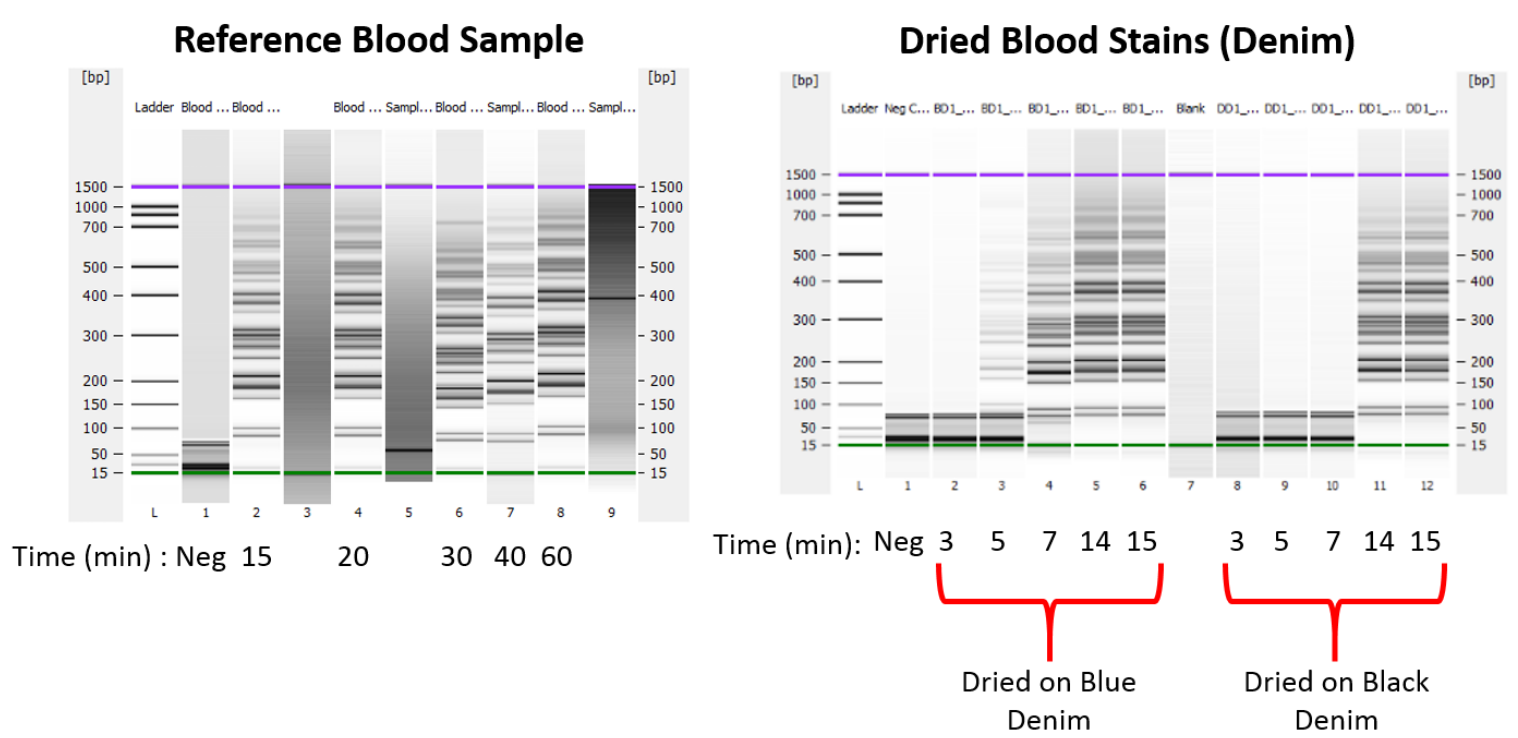

Figure 3: Time monitoring of RT-LAMP amplifications from a whole blood (reference) sample versus dried blood stains on blue and black denim material.

a small $1 \mathrm{~mm} \times 1 \mathrm{~mm}$ square cutting of the dried stain, blood could be identified in all samples within 15 minutes. A comparative example between whole blood (reference control) to dried blood samples is shown in Figure 3.

Unknown dried mixtures containing combinations of blood, vaginal fluid, semen, and saliva were prepared to challenge the LAMP assays as a BFI panel. A series of 8 samples were prepared and blindly tested and interpreted by a second user. The sample interpretation of these

Table 4: Blind Body Fluid Study Results

\begin{tabular}{|c|c|c|c|c|c|}
\hline Sample & Blood & $\begin{array}{c}\text { Vaginal } \\
\text { Fluid }\end{array}$ & Semen & Saliva & Score \\
\hline 1 & + & - & + & - & $\checkmark$ \\
\hline 2 & - & - & - & + & $\checkmark$ \\
\hline 3 & + & + & + & - & $\checkmark$ \\
\hline 4 & + & - & - & + & $\checkmark$ \\
\hline 5 & + & + & - & + & $\checkmark$ \\
\hline 6 & - & + & - & - & $\checkmark$ \\
\hline 7 & + & + & - & - & $\checkmark$ \\
\hline 8 & + & - & - & - & $\checkmark$ \\
\hline
\end{tabular}

blind samples, shown in Table 4 (primer sequences shown in Table 5A (appendix)), correctly matched all of the sample preparation and could be identified within a 30 minute amplification. 


\subsubsection{Sample Image Analysis Development}

Conventional enzymatic and chemical testing methods are popular due to the rapid sampling time, inexpensive reagents, and clear visual colorimetric readout, which were taken in account for the development of this assay. The turbidity of conventional LAMP amplifications can be difficult to decipher without fluorescence due to the low contrast of the turbidity relative to the background ${ }^{22}$, therefore, a colorimetric approach with hyroxynaphthol blue (HNB) was explored.

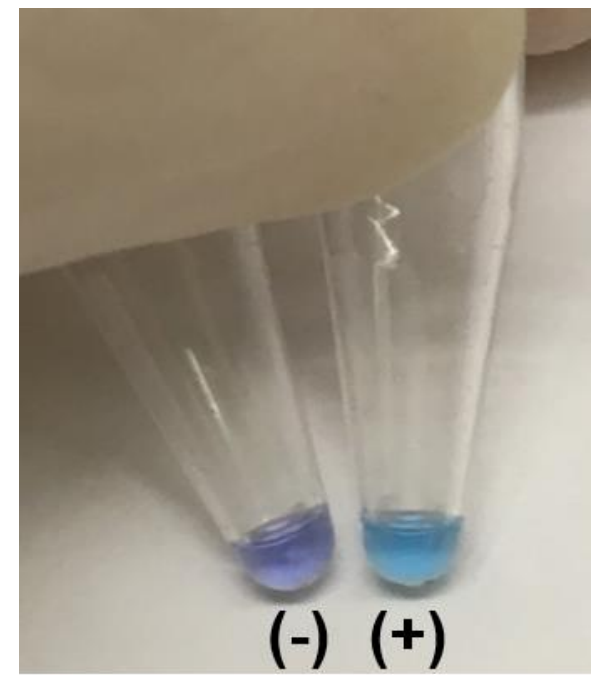

Figure 4: Picture of LAMP reaction positive and negative control with HNB dye

HNB dye has previously been used with LAMP reactions and described as being optimal at a concentration of $120 \mu \mathrm{M}^{22-25}$. A purple color, indicative of the chelation of $\mathrm{Mg}^{2+}$ by $\mathrm{dNTPs}^{26}$, changes to a sky blue color as the concentration of free $\mathrm{Mg}^{2+}$ in solution depletes in solution with amplification (see Fig 4). Sensitivity studies with this dye indicate that visual detection can occur with single copies of nucleic material which is useful for visual detection ${ }^{27,28}$.

A HNB disodium salt was first used as the colorimetric dye, as there were no description beyond the name of the HNB dye in any of the literature. The dye was first optimized for mock LAMP samples to obtain the greatest discrimination between a positive and negative control sample. To do this, PCR tubes were filled with 5-25 $\mu \mathrm{L}$ of the $\mathrm{HNB}$ dye in either $3 \mathrm{mM} \mathrm{MgCl}_{2}$ (negative control) or deionized water (positive control). The PCR tubes were imaged on the HAA imaging platform from Chapter 2 and run through a custom hue analysis algorithm that was previously described ${ }^{29}$ for comparison. The optimal HNB concentration was found to be approximately $30 \mathrm{mM}$, as $20 \mathrm{mM}$ was too light in color to visually see and $40 \mathrm{mM}$ HNB did not 

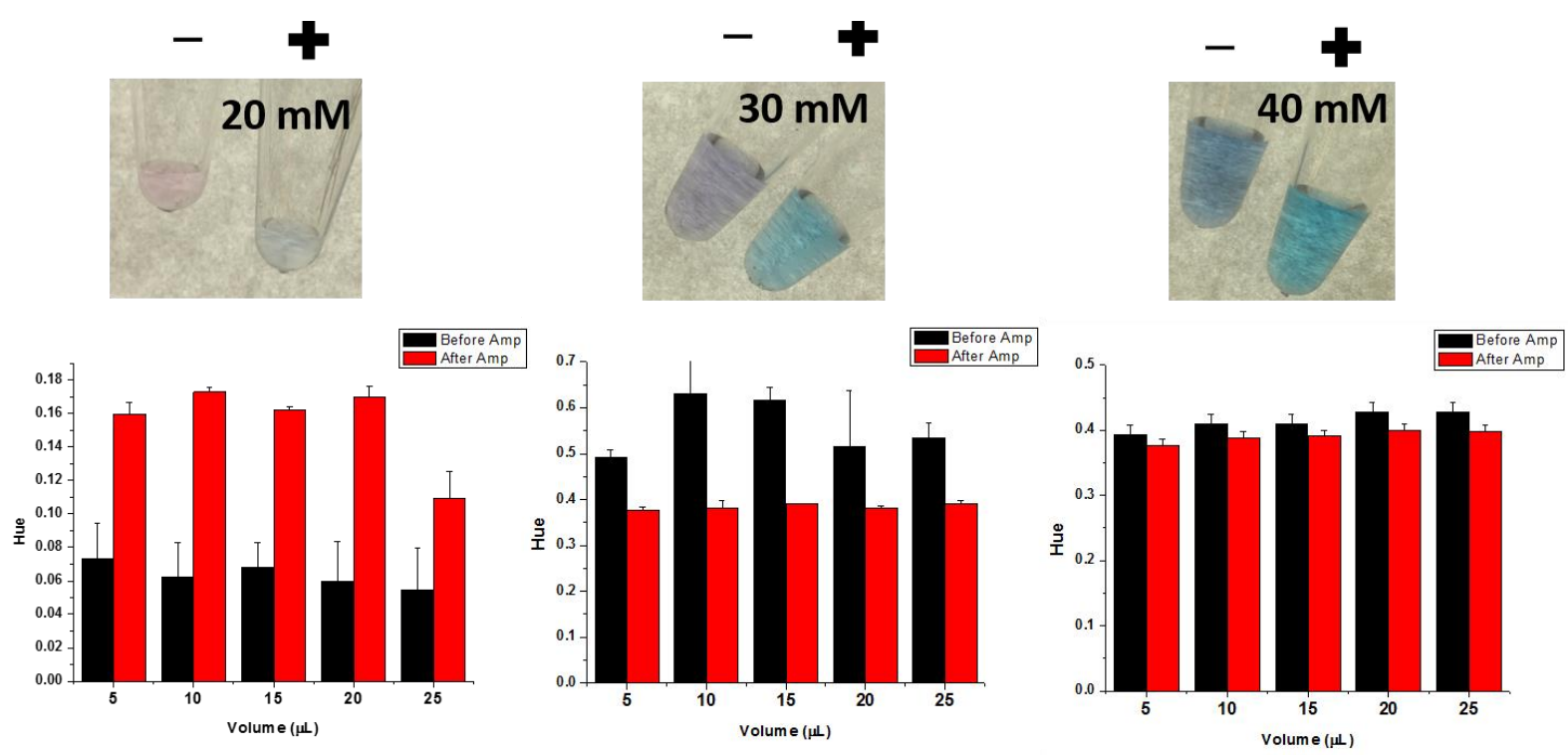

Figure 5: Optimization of HNB Disodium Salt Dye in Mock LAMP Reactions. An optical camera captured images of the solutions in the PCR tubes, which could be used for image analysis via a custom hue algorithm.

provide a distinguishable difference between a positive and a negative sample (Fig 5). When the dye was diluted to $120 \mu \mathrm{M}$ in a LAMP reaction, the color of the solution was no longer visible, which did not agree with previous literature. When all LAMP reactions failed to amplify with the HNB dye, experiments conditions were carefully checked with those listed in previous literature. After confirming that experimental conditions matched, it became very clear that either the described procedures were not written clearly or that there was a problem with the HNB dye.

After troubleshooting this challenge without any progress, further research was done on the structure of the HNB dye itself. Despite the dye having the same name as written in literature, the HNB disodium salt was found to be different than the dye used in the previously reported LAMP reactions. Instead of using the disodium salt, a metal indicator dye was used which had minimal chemical structure differences when compared (Figure 6). Despite these minor differences, the new dye provided a bright violet color at $120 \mu \mathrm{M}$ which was consistent with literature. When this dye was embedded in a LAMP reaction at $120 \mu \mathrm{M}$, the colorimetric change 
Hydroxy Naphthol Blue Disodium Salt

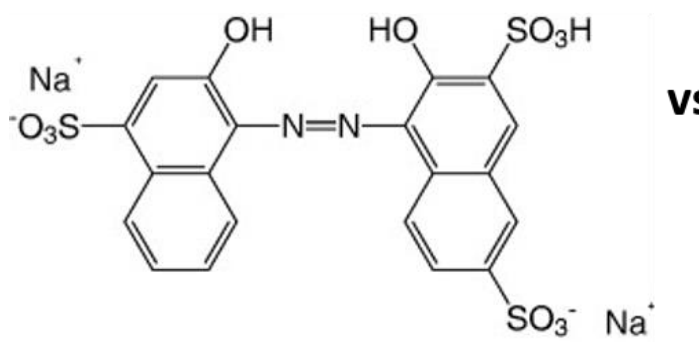

\section{Hydroxy Naphthol Blue Metal Indicator}

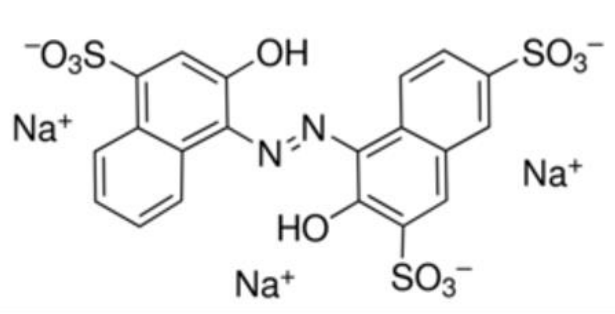

Figure 6: Comparison of HNB disodium salt chemical structure to the HNB metal indicator dye structure

from violet to sky blue was observed without negatively affecting the LAMP reaction. With the HNB dye now working with LAMP samples, the project was able to move forward with developing a protocol for image analysis.

To accommodate the maximum number of samples, colorimetric LAMP detection was adapted to a 96-well plate format. A rectangular case was made to fit a 96-well plate could fit directly in the top of the box (Fig. 7) with a cell phone placed below to take an image. The color

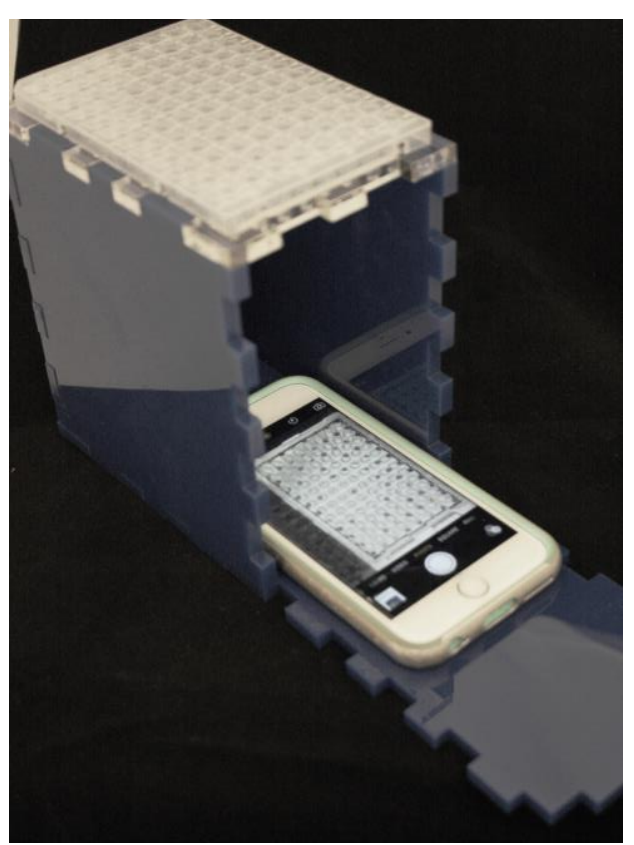

Figure 7: An image of the PMMA box used for 96-well plate imaging of LAMP samples of the well will help determine what body fluids are present but it was unknown whether the color could be differentiated by cell phone analysis. To test this, a testing plate was filled with mock LAMP samples (15 $\mu \mathrm{L}$ volume) using either nuclease-free water or $8 \mathrm{mM} \mathrm{MgSO}_{4}$ solution with $120 \mu \mathrm{M} \mathrm{HNB}$ to mimic the violet and sky blue colors before and after amplification, respectively. Using ImageJ software, 
A
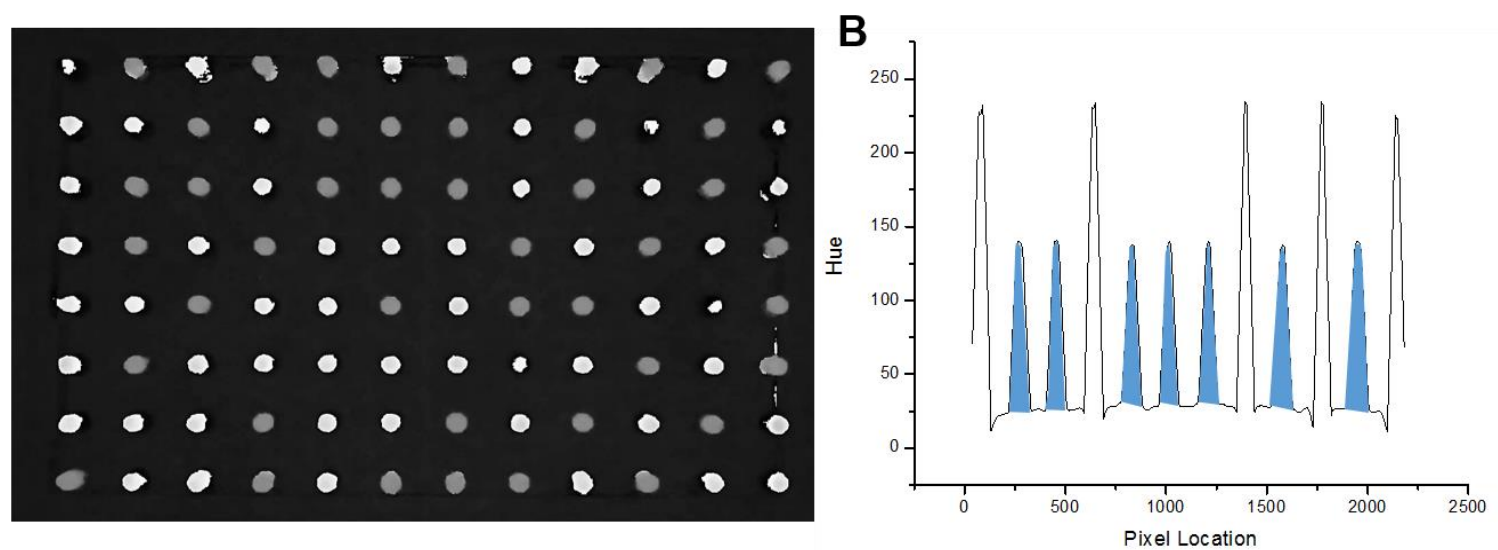

Figure 8: Cell Phone Imaging of LAMP Plate. (A) Cell phone image of 96-well plate containing mock LAMP samples that has been converted to the hue channel via Imagej. (B) Surface plots of the third row of the 96-well plate from panel (A) with amplified LAMP samples highlighted. The hue distribution of the negative and positive LAMP samples are plotted.

the hue for each well were plotted. All violet samples provided hue values between 170-220 whereas blue samples were below 150 (see Figure 8).

Multiple photos of this plate provided similar hue profiles with slight variability, but clearly differentiates between positive and negative samples with a threshold hue of 150 (see Figure 9). Cell phone analysis of a second plate looked at the minimal volume required for analysis as well as the interpretation of blank wells. The plate was filled with three sets of 5 , 10 , and $15 \mu \mathrm{L}$ positively and negatively mock LAMP samples that were separated by a blank well. The cell phone analysis

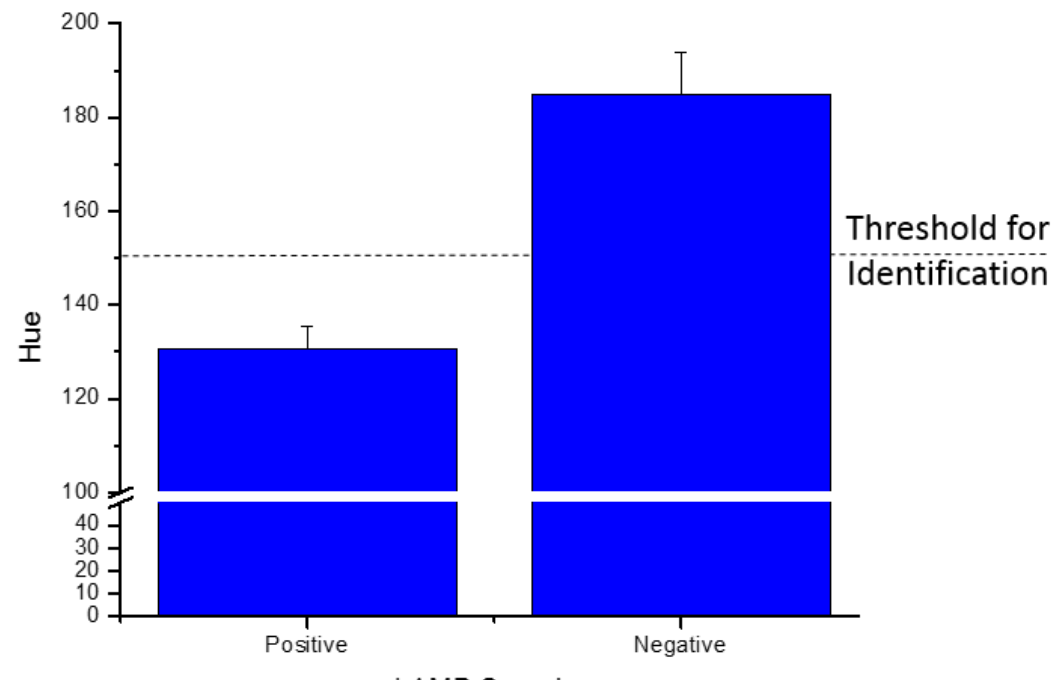
could clearly distinguish

Figure 9: Graph displaying the hue distribution of the negative and positive LAMP samples from a full 96 well plate. 


\section{LAMP Volume}
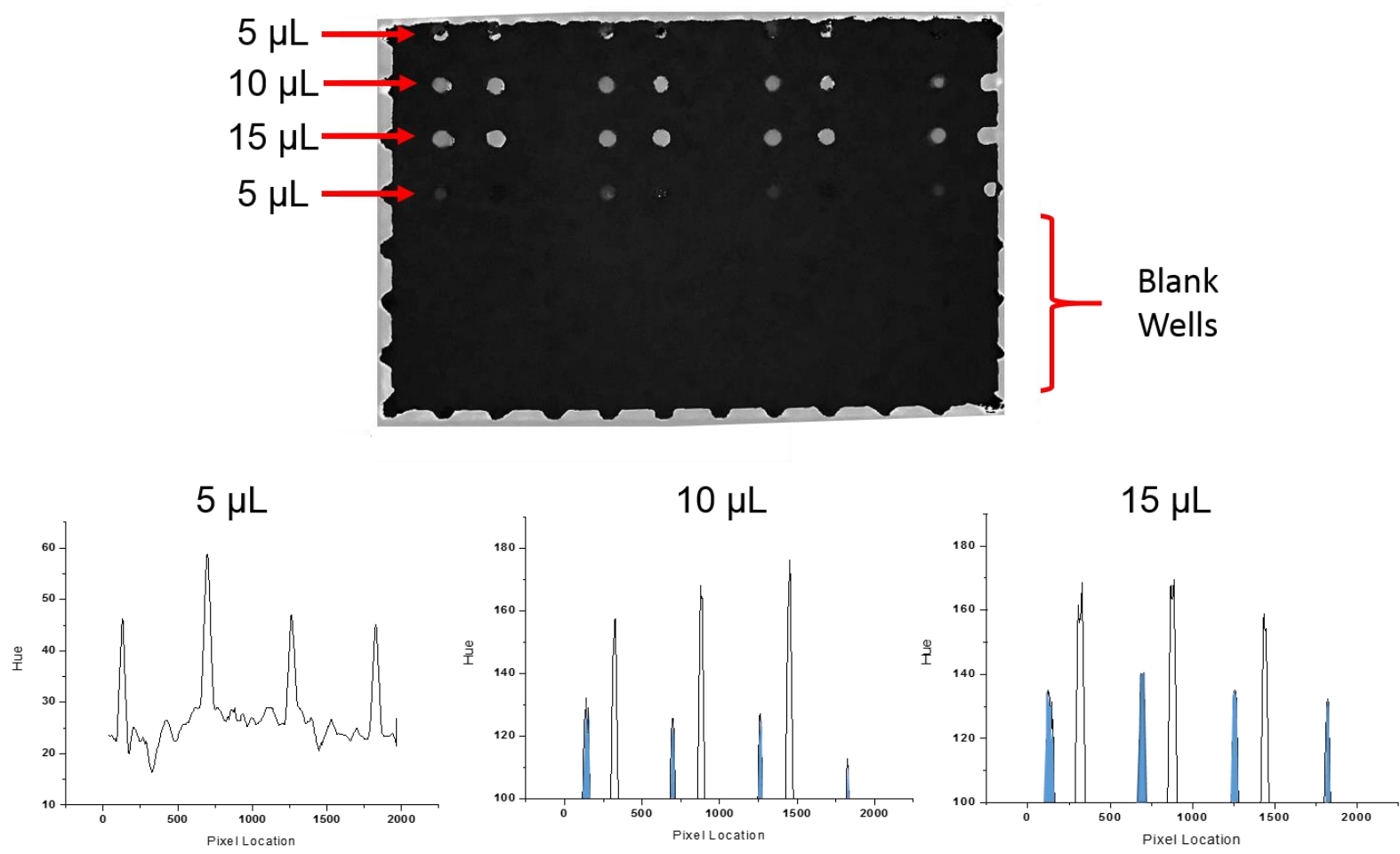

Figure 10: Picture of 96-well plate with 5, 10, and $15 \mu \mathrm{L}$ LAMP volumes with corresponding surface plots for each LAMP volume.

samples down to $10 \mu \mathrm{L}$. Below this volume, the blue LAMP samples dropped out in the profiles (see Figure 10). Any blank wells in the 96-wells plate blended with baseline hue values and did not interfere with the optical detection analysis.

\subsubsection{Challenging Samples}

As SEMG1 is a prominent protein in semen, we explored whether the semen LAMP assay was sensitive enough to identify azoospermatic semen samples. To do this, duplicate samples from three separate donors were processed and amplified alongside a negative control (containing only TE buffer) and a non-specific control (1 ng human genomic DNA). All of the semen samples could be correctly identified within 30 minutes via cell phone imaging, as shown in Figure 11. 

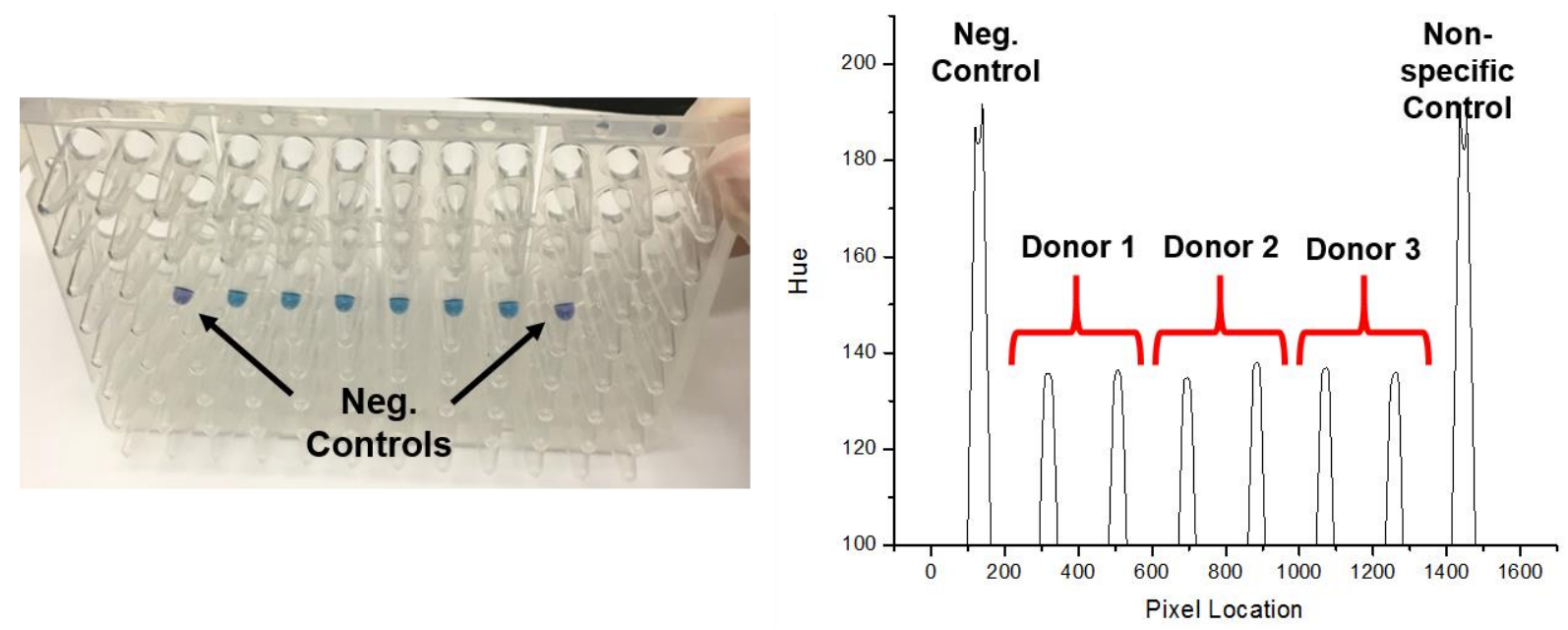

Figure 11: Picture of 96-well plate with corresponding image analysis of azoospermatic semen samples. Duplicate samples from three different donors were compared to a negative control (containing only TE buffer for sample) and a non-specific control (1 ng human genomic DNA).

This technique was further challenged to see whether a series of 23 sample mixtures (containing between 1-3 body fluids) could be correctly identified via LAMP and optical detection. The 96-well plate was organized into 4 sections, with 2 consecutive rows assigned to each target body fluid. Negative controls were automatically placed at the beginning of the first row as an internal plate standard and to ensure that none of the reagents were contaminated. After a 30 minute amplification, the 96-well plate was placed in the PMMA box and captured via a smart phone. Using ImageJ, the hue of unknown samples was compared to the negative controls. Only a hue value below the threshold of 150 will a sample be identified for containing the target body fluid. Mapping a surface plot of the hue along the entire plate provided a digital readout of all the body fluids present in all 23 samples simultaneously (Fig. 12). Fortunately, all samples were correctly identified and did not provide any false negatives or positives. 


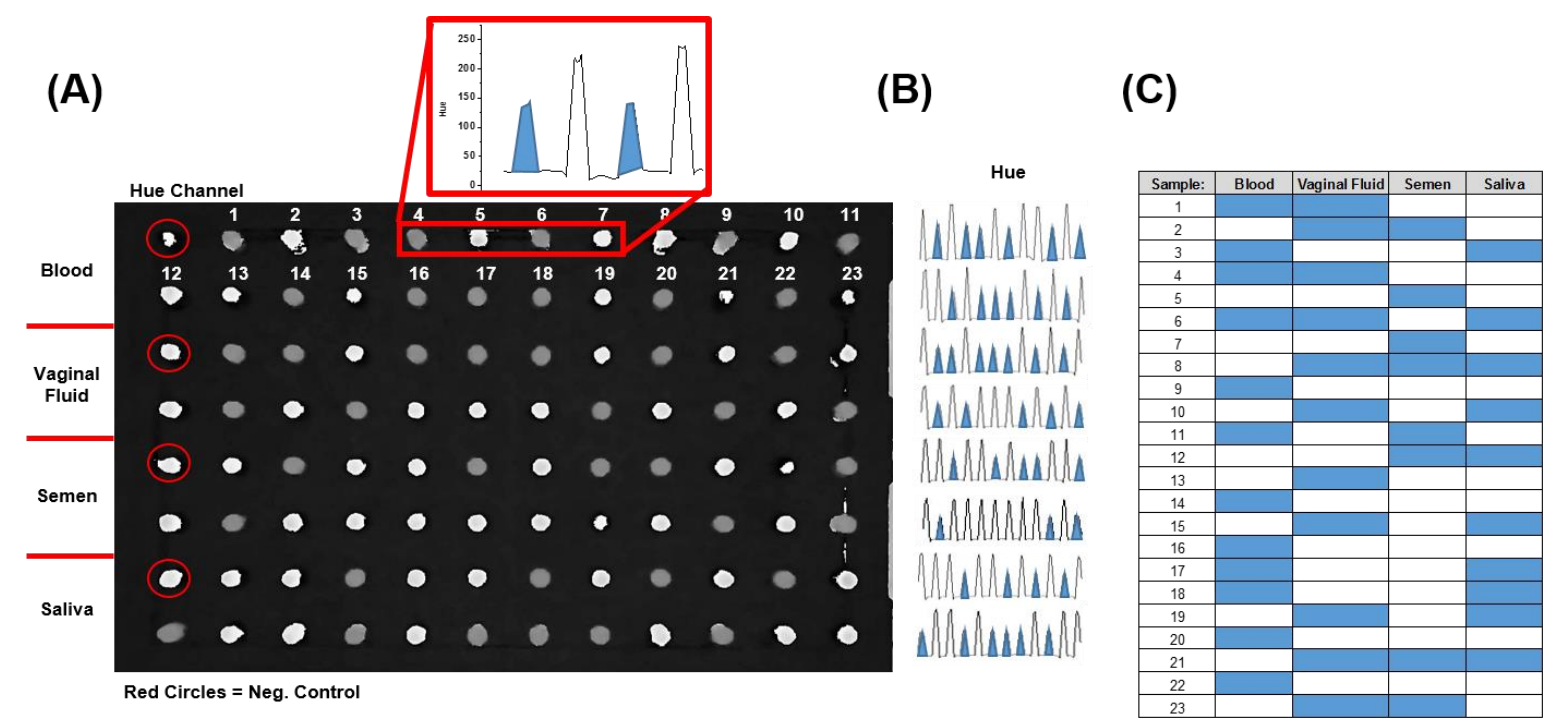

Figure 12: Cell Phone Image Analysis. (A) Cell phone image of 96-well plate containing mock LAMP samples that has been converted to the hue channel via Imagej. (B) Surface plots of each row of the 96-well plate from panel (A) with amplified LAMP samples highlighted. (C) Table summary of the body fluids contained in each sample analyzed in panel (A).

\subsection{Discussion}

LAMP provides many advantages over conventional PCR including high specificity, a large dynamic range, and single temperature amplification in a short 60 minute time frame. The high specificity of LAMP amplifications is derived from the four to six primers that recognize six to eight regions along a target sequence ${ }^{23}$. The ability for LAMP to discriminate between single nucleotides $^{30}$ minimizes the chances of encountering any false positives. In addition, the LAMP method is easily amendable to a simple workflow.

In this study we exploited the advantages of LAMP to create a body fluid panel that is simple to execute, provides rapid results, and does not require expensive detection instrumentation. The use of highly expressed mRNA markers with LAMP provided unique identification of each body fluid with no observed false-positives or negatives. This was significant, as previous studies used multiple fluid markers for BFI which were susceptible to false identification ${ }^{13}$. Using markers with lower fluid specificity led to the need of complex statistical algorithms to discriminate the 
presence of body fluids. However, with increased specificity via LAMP, the most specific mRNA marker can be used which eliminates the need for an algorithm.

A universal sample lysis and extraction method was developed and allowed the simultaneous processing and identification of all four body fluids within 30 minutes of amplification. The co-extraction of DNA and RNA prior to amplification allows analysts to do BFI testing in tandem with STR testing if desired. Unlike many reported methods for body fluid identification, this method does not require prior DNAse treatment and therefore is non-destructive to samples of interest. Regardless of the fluid mixture, this LAMP method was shown to be equally effective for blood, vaginal fluid, semen, and saliva samples which is imperative in unknown forensic mixtures. An exceptional dynamic range spanning over five orders of magnitude affords the detection of even trace samples which may be out of range for conventional enzymatic methods.

This LAMP method was applied to several fluid samples that were dried on difficult materials including denim and dark synthetic material. Initial testing of blood stains $(\sim 2.5 \mu \mathrm{L})$ showed sample amplifications within 15 minutes that was consistent between cotton, blue denim, and black denim stain samples. Vaginal fluid, saliva, and semen, on the other hand, were detectable after 30 minute amplifications. Only $25 \%$ of fluid stains $(\sim 2.5 \mu \mathrm{L})$ were consumed for BFI analysis. From this same extract, STR profiles could also be obtained, which left $75 \%$ of the stain for re-extracting if necessary. This sample consumption is significantly less than the $50 \mu \mathrm{L}$ stain samples previously reported ${ }^{13}$.

A visual colorimetric reaction, provided by a metal chelating dye embedded in the LAMP amplification, was easily captured with a smart phone camera and avoided the use of expensive and complicated instrumentation. The colorimetric change with HNB dye upon LAMP 
amplification is visually consistent with previous literature and does not inhibit the LAMP assays ${ }^{22,}$ 24, 26. A 96-well plate was used for high sample processing and could accommodate the testing of each body fluid (blood, vaginal fluid, semen, and saliva) with 23 samples and a negative control. One image can capture the full 96-well plate and be analyzed to provide a digital 'yes' or 'no' response based on the hue of each sample well. HNB dye was found to be stable in LAMP reactions and the 150 hue threshold was applicable for every plate tested. Empty sample wells did not interfere with sample analysis, allowing analysts freedom in running between $1-23$ samples simultaneously. Running a maximum of 23 samples could provide rapid sample-to-answer interpretation in under 3 hours.

\subsection{Conclusions}

Overall, this LAMP body fluid identification panel has significant potential for providing contextual information by accurately and rapidly identifying blood, vaginal fluid, saliva, and semen in unknown samples. Four highly discriminatory mRNA markers were used to design LAMP assays amplifiable at the same temperature. Specificity studies validated the accurate identification of each target body fluid which was reproducible between multiple users. The elegance of this approach is that it does not involve thermocycling and uses a simple dye for colorimetric read-out and a smart phone as a detector. Relative to existing methods, this provides an accelerated sample-to-answer method for mRNA with high specificity and sensitivity (single copies of RNA) and unparalleled bandwidth (5 fluids) for body fluid ID. This method was challenged with dry stains on denim material and azoospermatic samples which both performed remarkably well and could be successfully detected. A blind study also validated the efficacy of the method and demonstrated that the method can be used by individuals who are not highly 
trained. Future efforts will focus on automation of this system and validation of the method with real forensic samples. Other body fluids, such as urine, sweat, and menstrual blood could also be included in the assay in future experiments.

\subsection{References}

1. S. R. F. Harbison, Research and Reports in Forensic Medical Science, 2016, 2016:6, 11-23.

2. J. Butler, Advanced Topics in Forensic DNA Typing, Academic Press, 2011.

3. D. G. Casey and J. Price, Forensic Science International, 2010, 194, 67-71.

4. S. Johnston, J. Newman and R. Frappier, Canadian Society of Forensic Science Journal, 2003, 36, 173-183.

5. E. Diamandis and H. Yu, The Lancet, 1995, 345, 1186.

6. R. Nagar and K. K. Sharma, Indian Journal of Clinical Biochemistry, 2012, 27, 375-378.

7. U. Ricci, I. Carboni and F. Torricelli, Journal of forensic sciences, 2014, 59, 1410-1412.

8. D. Zubakov, E. Hanekamp, M. Kokshoorn, W. van IJcken and M. Kayser, International journal of legal medicine, 2008, 122, 135-142.

9. M. Setzer, J. Juusola and J. Ballantyne, Journal of forensic sciences, 2008, 53, 296-305.

10. J.-L. Park, S.-M. Park, J.-H. Kim, H.-C. Lee, S.-H. Lee, K.-M. Woo and S.-Y. Kim, Genomics Inform, 2013, 11, 277-281.

11. M. L. L. Richard, K. A. Harper, R. L. Craig, A. J. Onorato, J. M. Robertson and J. Donfack, Forensic Science International-Genetics, 2012, 6, 452-460.

12. C. Haas, B. Klesser, C. Maake, W. Baer and A. Kratzer, Forensic Science International-Genetics, $2009, \mathbf{3}, 80-88$.

13. P. Danaher, R. L. White, E. K. Hanson and J. Ballantyne, Forensic Science International: Genetics, $2015,14,18-30$. 
14. C.-W. Su, C.-Y. Li, J. C.-I. Lee, D.-D. Ji, S.-Y. Li, B. Daniel, D. Syndercombe-Court, A. Linacre and H.-M. Hsieh, Forensic Science Medicine and Pathology, 2015, 11, 208-215.

15. J. V. Norris, Doctoral Dissertation, University of Virginia, 2008.

16. D. Kimes and M. Tamir, Crime Laboratory Digest, 1985, 12, 32-33.

17. F. Fan, M. Yan, P. Du, C. Chen and B. Kan, Foodborne pathogens and disease, 2015, 12, 778-786.

18. L. Niessen, J. Luo, C. Denschlag and R. F. Vogel, Food microbiology, 2013, 36, 191-206.

19. K. A. Hagan, C. R. Reedy, J. M. Bienvenue, A. H. Dewald and J. P. Landers, Analyst, 2011, 136, 1928-1937.

20. H. Kaneko, T. Iida, K. Aoki, S. Ohno and T. Suzutani, Journal of clinical microbiology, 2005, 43, 3290-3296.

21. H. Nakanishi, T. Ohmori, M. Hara, A. Takada, H. Shojo, N. Adachi and K. Saito, Journal of forensic sciences, 2011, 56.

22. J. Fischbach, N. C. Xander, M. Frohme and J. F. Gloekler, Biotechniques, 2015, 58, 189-194.

23. N. Tomita, Y. Mori, H. Kanda and T. Notomi, Nat. Protocols, 2008, 3, 877-882.

24. J. Hadersdorfer, M. Neumuller, D. Treutter and T. C. Fischer, Annals of Applied Biology, 2011, 159, 456-466.

25. K. W. Soli, M. Kas, T. Maure, M. Umezaki, A. Morita, P. M. Siba, A. R. Greenhill and P. F. Horwood, Diagnostic Microbiology and Infectious Disease, 2013, 77, 321-323.

26. M. Goto, E. Honda, A. Ogura, A. Nomoto and K. I. Hanaki, Biotechniques, 2009, 46, 167-+.

27. L. Luo, K. Nie, M. J. Yang, M. Wang, J. Li, C. Zhang, H. T. Liu and X. J. Ma, Journal of Clinical Microbiology, 2011, 49, 3545-3550.

28. R. Ghosh, A. Nagavardhini, A. Sengupta and M. Sharma, Bmc Research Notes, 2015, 8.

29. Y. Ouyang, J. Li, C. Phaneuf, P. S. Riehl, C. Forest, M. Begley, D. M. Haverstick and J. P. Landers, Lab on a Chip, 2016, 16, 377-387.

30. M. Iwasaki, T. Yonekawa, K. Otsuka, W. Suzuki, K. Nagamine, T. Hase, K.-I. Tatsumi, T. Horigome, T. Notomi and H. Kanda, Genome Letters, 2003, 2, 119-126. 


\section{Chapter 3 Appendix}

Table 1A: LAMP Primers for Blood

\begin{tabular}{|c|c|c|}
\hline Target & Primers & Sequence $\left(5^{\prime}-3^{\prime}\right)$ \\
\hline \multirow[t]{4}{*}{ Blood 1} & FIP & GCCAAAGTGATGGGCCAGCACCTGAGAACTTCAGGCTCCT \\
\hline & BIP & AAGAATTCACCCCACCAGTGCAAGGGCATTAGCCACACCA \\
\hline & F3 & GCTGCACTGTGACAAGCT \\
\hline & B3 & TGGACAGCAAGAAAGCGAG \\
\hline \multirow[t]{6}{*}{ Blood 2} & FIP & CCAGGAGCCTGAAGTTCTCAGGTTTTGGCACCTTTGCCACACTG \\
\hline & BIP & TGTGTGCTGGCCCATCACTTTTTTCCAGCCACCACTTTCTGAT \\
\hline & F3 & CTCACCTGGACAACCTCAAG \\
\hline & B3 & TTGTGGGCCAGGGCATTA \\
\hline & LF & TGCAGCTTGTCACAGTGCA \\
\hline & LB & AATTCACCCCACCAGTGCA \\
\hline \multirow[t]{6}{*}{ Blood 3} & FIP & CGTTGCCCAGGAGCCTGAAGTTTTACTGAGTGAGCTGCACTGT \\
\hline & BIP & GGTCTGTGTGCTGGCCCATCTTTTCCAGCCACCACTTTCTGAT \\
\hline & $\mathrm{F} 3$ & CCTCAAGGGCACCTTTGC \\
\hline & B3 & TTGTGGGCCAGGGCATTA \\
\hline & LF & AGGATCCACGTGCAGCTTGT \\
\hline & LB & CTTTGGCAAAGAATTCACCC \\
\hline \multirow[t]{6}{*}{ Blood 4} & FIP & AGCCTTCACCTTAGGGTTGCCTTTTTGAGTCCTTTGGGGATCTGT \\
\hline & BIP & CATGGCAAGAAAGTGCTCGGTGTTTTGTGCCCTTGAGGTTGTCC \\
\hline & F3 & CTGGTGGTCTACCCTTGGA \\
\hline & B3 & CAGCTCACTCAGTGTGGC \\
\hline & LF & CATAACAGCATCAGGAGTGG \\
\hline & LB & TTAGTGATGGCCTGGCTCA \\
\hline
\end{tabular}


Table 2A: LAMP Primers for Vaginal Fluid

\begin{tabular}{|c|c|c|}
\hline Target & Primers & Sequence $\left(5^{\prime}-3^{\prime}\right)$ \\
\hline \multirow[t]{6}{*}{$\begin{array}{l}\text { Vaginal } \\
\text { Fluid } 1\end{array}$} & FIP & CCACCTGAGGCCATCTCAGACATTTTCCAGTCGCCATGAGAACTTC \\
\hline & BIP & ACTTTCTCACAGGCCTTGGCCTTTTGAGACATTGCCCTCCACTG \\
\hline & F3 & CCTGAAATCCTGGGTGTTGC \\
\hline & B3 & AAGATCGGGCAGGCAGAA \\
\hline & LF & AGCAGAGAGTAAACAGCAGAA \\
\hline & LB & GATCTGATCATTACAATTGCG \\
\hline \multirow[t]{6}{*}{$\begin{array}{l}\text { Vaginal } \\
\text { Fluid } 2\end{array}$} & FIP & GGCAACACCCAGGATTTCAGGATTTTCTGGAAGCCTCTGTCAGC \\
\hline & BIP & TGCCAGTCGCCATGAGAACTTTTTTCCTGAGGCCATCTCAGACAA \\
\hline & F3 & TACAGTGACGCTCCAGCC \\
\hline & B3 & GGCCTGTGAGAAAGTTACCA \\
\hline & LF & AGACGCTGGCTCCTTTGGA \\
\hline & LB & CCTTCTGCTGTTTACTCTCTGCT \\
\hline
\end{tabular}


Table 3A: LAMP Primers for Semen

\begin{tabular}{|c|c|c|}
\hline Target & Primers & Sequence $\left(5^{\prime}-3^{\prime}\right)$ \\
\hline \multirow[t]{6}{*}{ Semen 1} & FIP & AGATCCATGTTGGTGTCTGCCTTTTCAATCTACAAATAGAGAACAAGACC \\
\hline & BIP & TAGAGCAGGAAGATGACAGTGATTTTATAATGGGTTTCGGTCGT \\
\hline & F3 & GCAAAAGGAGAGTCTGGC \\
\hline & B3 & GGTTACCGAATGGTAGGTT \\
\hline & $\mathrm{LF}$ & TTTGTTCATGACTGAGTA \\
\hline & LB & GTCATTTGGCACAACATCTT \\
\hline \multirow[t]{5}{*}{ Semen 2} & FIP & GTTTCGGTCGTTGTTAAGATGTTGTTTTTTAATTATAGAGCAGGAAGATGACAG \\
\hline & BIP & TAAACCTACCATTCGGTAACCATGTTTTCACTGAGGTCAACTGACA \\
\hline & F3 & TCTCATGGGGGATTGGAT \\
\hline & B3 & CATCTCAGAAACATCACAGAA \\
\hline & LB & GAAAGGATGGACCAATATCAAG \\
\hline \multirow[t]{6}{*}{ Semen 3} & FIP & GGTCAACTGACACCTTGATATTGGTTTTTTTACATAAACCTACCATTCGGTA \\
\hline & BIP & GAGATGCAGACTCCCGTGTATTTTAAAGCCTAATTCAAGGAAGC \\
\hline & F3 & CAACATCTTAACAACGACCG \\
\hline & B3 & TCTCTTTATTGGAAGTTTGAAGG \\
\hline & $\mathrm{LF}$ & CCATCCTTTCACATGGT \\
\hline & LB & TCAGATTCTTGGTCCATGGAT \\
\hline \multirow[t]{6}{*}{ Semen 4} & FIP & ATGTCTTTGGATCCATGTTGGAGTTTTTTGAACATTCAAGTAAAGTACAAACC \\
\hline & BIP & ACCCAAGATGAGCTCCTAGTATATTTTTCCATGCTGTTGATCTTGATTG \\
\hline & F3 & TGTGGTTGAAGTGAGAGAG \\
\hline & B3 & ATGATATTTTATTTGCCTTTCGG \\
\hline & $\mathrm{LF}$ & TCTTGGTGCGCAGGACAGA \\
\hline & LB & CAAGAATCAACACCAGAC \\
\hline
\end{tabular}


Table 3A: LAMP Primers for Semen Continued

\begin{tabular}{|c|c|c|}
\multicolumn{1}{c|}{ Target } & Primers & Sequence $\left(5^{\prime}-3^{\prime}\right)$ \\
\hline Semen 5 & FIP & TAGGCTTCCCAGTGCCTCCAGTTTTCCAGACACTCACAGCAAGG \\
\hline & BIP & TTCCTTTGGCATGGGATGGGGTTTTACCTCCCCCCATAGTGAAT \\
\hline & F3 & CCATGAAGCACTGAGCAGAA \\
\hline & B3 & AGGGTTGTCTGGAGGACTTC \\
\hline & LF & GAGTGGGTTATGTTTTCAGC \\
\hline & LB & AAGTAAGGAGAGGGACTGGAC \\
\hline
\end{tabular}

Table 4A: LAMP Primers for Saliva

\begin{tabular}{|c|c|c|}
\hline \multicolumn{1}{|c|}{ Target } & Pequence $\left(5^{\prime}{ }^{-3}{ }^{\prime}\right)$ \\
\hline Saliva 1 & FIP & GATGTGAATGATGCTTTTCATGGAATTTTCTGGAGCTGATTCACATGC \\
\hline & BIP & ATTGATATCTTCAGTAATCACGGGGTTTTAGTCCAAAGCGAATTTGC \\
\hline & F3 & TTGGCTCTCATGCTTTCC \\
\hline & B3 & GGTATGACAAATGAGAATACACG \\
\hline & LF & TATACCCATGATGTCTCT \\
\hline Saliva 2 & FIP & GTTGGCTGAGTCCTTTTACTCAATTTTGAGACTTCACTTCAGCTTCA \\
\hline & BIP & TTGTTTTTGCTTTAATCTTGGCTCTTTTTTTTTATACCCATGATGTCTCTTTG \\
\hline & F3 & CCATCTAGGAAAGGACATCTC \\
\hline & B3 & TCTATAGCCTCGATGTGAATG \\
\hline & LF & GAGGAGAATCCAGAAGTCAG \\
\hline & LB & CCATGACTGGAGCTGATTCAC \\
\hline
\end{tabular}


Table 5A: Blind Body Fluid Study Primers

\begin{tabular}{|c|c|c|}
\hline Target & Primers & Sequence $\left(5^{\prime}-3^{\prime}\right)$ \\
\hline \multirow[t]{4}{*}{ Blood } & FIP & GCCAAAGTGATGGGCCAGCACCTGAGAACTTCAGGCTCCT \\
\hline & BIP & AAGAATTCACCCCACCAGTGCAAGGGCATTAGCCACACCA \\
\hline & F3 & GCTGCACTGTGACAAGCT \\
\hline & B3 & TGGACAGCAAGAAAGCGAG \\
\hline \multirow[t]{6}{*}{$\begin{array}{l}\text { Vaginal } \\
\text { Fluid }\end{array}$} & FIP & GGCAACACCCAGGATTTCAGGATITTCTGGAAGCCTCTGTCAGCT \\
\hline & BIP & TGCCAGTCGCCATGAGAACTTIITTCCTGAGGCCATCTCAGACAA \\
\hline & F3 & TACAGTGACGCTCCAGCC \\
\hline & B3 & GGCCTGTGAGAAAGTTACCA \\
\hline & $\mathrm{LF}$ & AGACGCTGGCTCCTITGGA \\
\hline & LB & ССTTCTGCTGTTTACTCTCTGCT \\
\hline \multirow[t]{6}{*}{ Semen } & FIP & AGATCCATGTTGGTGTCTGCCTTTTCAATCTACAAATAGAGAACAAGACC \\
\hline & BIP & TAGAGCAGGAAGATGACAGTGATTTTATAATGGGTTTCGGTCGT \\
\hline & F3 & GCAAAAGGAGAGTCTGGC \\
\hline & B3 & GGTTACCGAATGGTAGGTT \\
\hline & $\mathrm{LF}$ & TाTGTTCATGACTGAGTA \\
\hline & LB & GTCATTTGGCACAACATCTT \\
\hline \multirow[t]{6}{*}{ Saliva } & FIP & GATGTGAATGATGCTITTCATGGAATITTCTGGAGCTGATTCACATGC \\
\hline & $\mathrm{BIP}$ & ATTGATATCTTCAGTAATCACGGGGTTITAGTCCAAAGCGAATTTGC \\
\hline & F3 & TTGGCTCTCATGCTTTCC \\
\hline & B3 & GGTATGACAAATGAGAATACACG \\
\hline & $\mathrm{LF}$ & TATACCCATGATGTCTCT \\
\hline & LB & CATGATTATGGAGGTITGAC \\
\hline
\end{tabular}




\section{DNA Purification using Dynamic Solid-Phase Extraction on a Rotationally-driven Polyethylene-Terephthalate Microdevice}

\section{Summary}

The development of a disposable polyester toner centrifugal device is reported for semiautomated, dynamic solid phase DNA extraction (dSPE) from whole blood samples. The integration of a novel adhesive and hydrophobic valving with a simple and low cost microfabrication method allowed for sequential addition of reagents without the need for external equipment for fluid flow control. The spin-dSPE method yielded an average extraction efficiency of $\sim 45 \%$ from $0.6 \mu \mathrm{L}$ of whole blood. The device performed single sample extractions or accommodate up to four samples for simultaneous DNA extraction, with PCR-readiness DNA confirmed by effective amplification of a $\beta$-globin gene. The purity of the DNA was challenged by a multiplex amplification with 16 targeted amplification sites. Successful multiplexed amplification could routinely be obtained using the purified DNA collected post an on-chip extraction, with the results comparable to those obtained with commercial DNA extraction methods. This proof-of-principle work represents a significant step towards a fully-automated low cost DNA extraction device and is presented in this chapter as well as Jackson et al. ${ }^{1}$

\subsection{Introduction}

DNA extraction is a crucial step in genetic analysis as this directly affects downstream processing of DNA, including accurate DNA quantitation and amplification using the polymerase chain reaction $(\mathrm{PCR})^{2-5}$. Current commercial kits exploit two primary forms of DNA extraction: those containing packed silica columns (solid phase extraction (SPE)) and others with silica-coated magnetic particles freely moving, which are 
controllable by external magnets (dynamic solid phase extraction (dSPE)). In both cases, the extraction chemistry remains the same to yield purified, readily-amplifiable DNA through binding of DNA to beads followed by washing and elution. dSPE is advantageous over SPE because it circumvents issues associated with the reproducible packing of solid phases, and allows for thorough interaction of DNA with the silica surface of the beads during binding and elution ${ }^{6,7}$.

Translating DNA extraction to a microfluidic platform in a manner that allows for high quality DNA extraction from raw samples provides significant advances over conventional benchtop instrumentation ${ }^{2,4,5,8,9}$. These advantages, largely tethered to the miniaturization, include the potential for: $(i)$ reduced cost with decreasing reagent and sample volume ${ }^{10-13}$; (ii) enclosed devices for decreased risk for contamination with the development of integrated steps, and, consequently, reduced analyst manipulation; (iii) total automation ${ }^{10-13}$; (iv) portability, and, finally, ( $\left.v\right)$ operation outside of complex and expensive laboratories ${ }^{10,12-14}$.

Among all microfluidic platforms, rotationally-driven microfluidics provide a simple and robust method for DNA extraction, as transporting liquids can be done by simply controlling spin speed, which presents the potential for integrating and automating all aspects of the extraction process. Several elegant methods for DNA extraction have been demonstrated in a single chamber ${ }^{15,16}$, however, these methods are limited by the use of bulky external syringe pumps and valve systems. In contrast, rotation-driven systems rely on centrifugation as the sole force driving fluid flow through the microfluidic device, circumventing hydraulic systems (e.g., syringe pumps) and the associated external hardware that leads to more complex instrumentation ${ }^{14,17-20}$. 
Sequential addition of reagents in DNA extraction is essential to successful purification; therefore, centrifugal microdevices have to be coupled to a mechanism that controls fluidic movement. This is not a novel concept, as a combination of architectural flow restraints, valves, and rotational speeds can be used to control liquid in centrifugal microfluidics, as liquid is driven radially from the center outwards ${ }^{14}, 17,18,20$. Most centrifugal devices for DNA extraction utilize substrates including poly(methyl methacrylate) (PMMA), polycarbonate (PC), and cyclic olefin copolymer (COC), all of which use high rotation speeds $(>33 \mathrm{~Hz})$ due to surface tension ${ }^{21-24}$. For these extraction devices, external valving systems, such as a mechanical laser valves ${ }^{21}$, hydraulic capillary valves following surface treatment ${ }^{23}$, or the use of a stacked magnet interface (to allow bead transfer between static extraction solutions) $)^{22,24}$, have been exploited. All of the devices described in the literature have targeted large-volume samples (>100 $\mu \mathrm{L}$ ), excluding some important smaller-volume applications. Although all of these devices claim to extract DNA, only one of these methods using a complex stacked magnet ${ }^{22,24}$ yields purified DNA that is PCR-ready.

Unlike other microfluidic substrates, polyethylene terephthalate $(\mathrm{Pe})$ is commercially available with a hydrophilic coating that enhances capillary flow which presents an opportunity for coupling passive valving with the ease of the print-cut-laminate (PCL) fabrication methodology for on-chip purification ${ }^{10,11,25,26}$. Duarte et al. described a dSPE-based DNA extraction on Pe-toner (PeT) devices ${ }^{10}$ and recovered roughly $34 \%$ of DNA from $0.6 \mu \mathrm{L}$ of whole blood that was high-quality and compatible with PCR amplification. Despite the advantages offered by this approach, the method was very labor intensive, requiring several manual pipetting and mixing steps, which not only increased 
the risk for contamination, but also significantly extended the extraction time and demonstrated that this approach was not likely applicable to multiplexing or higher throughput.

While the need for automating the PeT device-based dSPE process is evident, several challenges exist, with flow control being a significant one. Ouyang et al. described a simple and cost-effective microfabrication method for implementing hydrophobic barrier valves using laser-printed toner in a multilayer PeT device ${ }^{27}$. While effective with aqueous solutions, toner valves are incompatible with organic solvents (e.g., alcohol) which compromises the full integration of the dSPE process. Furthermore, toner valves are onetime actuated valves (i.e., non-reversible), and once actuated, a downstream valve remains open for all subsequent steps $^{28}$. The irreversible nature of the toner valve introduces challenges of separating the purified DNA from the waste in the final stages of an on-chip extraction process.

With the aim of demonstrating proof-of-principle multi-step DNA extraction process on a spin device, this chapter describes a multi-sample spin-dSPE microdevice capable of yielding high quality, purified DNA from whole blood for PCR amplification. The PCR-readiness is established via successful amplification of both with single and multiple amplicons. Additionally, to address the need for alternative valving, the 'tape valve' described by Lounsbury et al. ${ }^{29}$ on a PMMA microdevice, is modified to allow for a semi-reversible valve that does not rely on a hydrophobic barrier. 


\subsection{Materials and Methods}

\subsubsection{Reagents}

Guanidine Hydrochloride (GuHCl) (MP Chemicals, Santa Ana, CA) was dissolved in deionized water to concentrations of $6 \mathrm{M}$ and $3 \mathrm{M}$ and adjusted to $\mathrm{pH}$ 6.1. Magnesil® paramagnetic particles (Promega, Madison, WI) were then diluted 3.3-fold in the $3 \mathrm{M}$ $\mathrm{GuHCl}$ solution prior to extractions. An $80 \%$ (wt/wt) isopropanol (IPA) (Sigma) solution and a $1 \mathrm{X}$ Tris-EDTA (TE) buffer (pH 8.0; Sigma Aldrich, St. Louis, MO) were prepared in deionized water prior to extractions. Picogreen (Invitrogen, Carlsbad, CA) was used as the intercalating dye for all DNA quantitation following extractions. All further reagents mentioned are without modifications. All pH adjustments were performed using a Mettler Toledo MP 220 pH meter.

\subsubsection{Device design and fabrication}

All microfluidic structures and architecture were designed using CAD software (AutoCAD; Autodesk Inc., San Rafael, CA). Commercially available Pe transparency sheets (Film Source Inc, Maryland Heights, MO) were used to fabricate the devices using a previously described 'print, cut, laminate' method $^{10,26,27}$. Briefly, device architecture was cut into toner printed or patterned Pe transparency sheets prior to layer assembly and adhesive bonding via thermal lamination. The lamination procedure was modified so that the assembled microdevice was sandwiched between pre-cut pieces of $\sim 1 \mathrm{~mm}$ thick brass shimstock.

Each device accommodates four extractions, with channel thicknesses of $272 \mu \mathrm{m}$. A circular $6.19 \mathrm{~mm}$ polydimethylsiloxane (PDMS) film (125 $\mu \mathrm{m}$ thick) was attached to the 
top of the recovery chamber in each extraction domain using laser-ablated pressure sensitive adhesive (PSA) (ARcare 90106; Adhesives Research Inc, Glen Rock, PA). The PDMS films were prepared according to manufacturer instructions in a Sylgard kit (Dow Corning, Midland, MI), using a 1:10 ratio of curing agent to polymer. The PDMS polymer, once degassed, was then poured onto a silicon wafer substrate and spun in a spincoater (model WS-400-6NPP-Lite; Laurell Technologies, North Wales, PA) to achieve a $125 \mu \mathrm{m}$ thickenss (verified by extrinsic fabry-perot interferometry (EFPI) using a Fiberpro USB interferometer (Luna Innovations, Roanoke, VA)). Transparent $19 \mathrm{~mm}$ thick adhesive tape (Scotch Magic ${ }^{\text {TM }}$ Tape; 3M, St. Paul, MN) was then placed over the remaining $2.25 \mathrm{~mm}$ circular openings through the top transparency layer using a $2.25 \mathrm{~mm}-2.8 \mathrm{~mm}$ diameter circular cutting of PSA.

\subsubsection{Construction of a spin platform and real-time monitoring}

A spin system was built in-house to enable separate control of magnetic mixing and fluidic control. An 8-core microcontroller (Propeller P8X32A-M44; Propellar Inc, Rockland, CA) was used to power two separate stepper motors (Sanmotion series, Sanyo denki, Moriguchi, Osaka prefecture, Japan) with 1.8 degree resolution driven by a stepper motor driver (drv8801). Each motor holds a $1.5 \mathrm{~mm}$ poly(methyl methacrylate) (PMMA) disc and is controlled by a serial terminal interface provided from the manufacturer website (Parallax Inc). The top disc was inverted and designated for magnetic mixing. Permanent N42 Neodymium magnets (ND024-42NM, CMS Magnetics, Garland, TX) were adhered to the top 150 mm diameter PMMA disc using 5-minute epoxy (Cat 14250; Devcon, Inc; Danvers, MA). In each domain, the negative magnetic pole was placed $29 \mathrm{~mm}$ from the 
rotational center facing the microfluidic device, with the positive magnetic pole $10 \mathrm{~mm}$ from the sample device. The bottom disc was used for placement of a dSPE device and fluidic control. The distance between the PMMA discs were controlled via three stepper motors and limited between 7 and $49 \mathrm{~mm}$ apart.

A high speed camera (MotionBLITZ EoSens mini 1-1; Mikrotron, San Diego, CA), laser (SLS-115/230; Monarch Instrument, Amherst, NH) and stroboscope (Nova-strobe PBL; Monarch Instrument, Amherst, NH) setup was used for monitoring the fluidic movement through the microfluidic device in real-time.

\subsubsection{Sample Preparation for dSPE Extraction}

Blood samples with known white blood cell counts were provided by the University of Virginia Medical School and were prepared as previously described ${ }^{10}$. Briefly, $12 \mu \mathrm{L}$ of whole blood was combined in a mixture of $10 \mu \mathrm{L}$ Protinase K (Qiagen, Valencia, CA) and $18 \mu \mathrm{L}$ of $6 \mathrm{M} \mathrm{GuHCl}$ and incubated at $56{ }^{\circ} \mathrm{C}$ for $10 \mathrm{~min}$. Aliquots of $2 \mu \mathrm{L}$ were used for each extraction, which is equivalent to $0.6 \mu \mathrm{L}$ whole blood.

\subsubsection{DNA Extraction Procedure}

All reagents were initially loaded onto the device prior to on-chip extraction: $6 \mu \mathrm{L}$ TE buffer, $4 \mu \mathrm{L}$ IPA (each chamber), $3 \mu \mathrm{L}$ of magnetic silica particle solution, $2 \mu \mathrm{L}$ of $3 \mathrm{M}$ $\mathrm{GuHCl}$, and $2 \mu \mathrm{L}$ lysed sample. Once reagents were placed in the microdevice, a saved propeller automated program began the extraction process. Mixing of the beads to bind the DNA in the solution was initiated with the stepper motors bringing the magnets, stationary on the magnetic disc, $10 \mathrm{~mm}$ from the sample disc. The sample disc was then swayed $43^{\circ}$ 
bi-directionally at a frequency of $0.736 \mathrm{~Hz}$ for $500 \mathrm{sec}$ for comprehensive mixing. Once the mixing was completed, the magnetic disc was brought to a height of $49 \mathrm{~mm}$ from the sample disc to start the fluidic control process.

The following were found to be optimal spin parameters after initial binding between DNA and silica: (i) IPA wash: $5 \mathrm{~Hz}, 110 \mathrm{sec}, 0.67 \mathrm{~Hz} \mathrm{sec}^{-1}$, clockwise; (ii) TE wash: 6.67 $\mathrm{Hz}, 10 \mathrm{sec}, 0.67 \mathrm{~Hz} \mathrm{sec}{ }^{-1}$, clockwise; (iii) TE elution: $11.67 \mathrm{~Hz}, 1 \mathrm{sec}, 0.67 \mathrm{~Hz} \mathrm{sec}{ }^{-1}$, clockwise; and finally (iv) transfer to the DNA recovery chamber: $13.33 \mathrm{~Hz}, 1 \mathrm{sec}, 0.67$ $\mathrm{Hz} \mathrm{sec}^{-1}$, counter-clockwise. After the TE wash was complete, the motor decelerated to a full stop to close the tape valve located between the binding chamber and the waste chambers. The DNA was then recovered from the device post-extraction by puncturing through the PDMS sleeve on the recovery chamber with a clean SGE liquid syringe (cat \# 24845; Restek, Bellefonte, PA).

\subsubsection{Conventional Solid Phase Extraction}

A Qiagen tube extraction served as a control method for the on-chip extraction (QIAamp DNA Mini Kit; Germantown, MD). This commercial kit was used to purify a 2 $\mu \mathrm{L}$ aliquot of each lysed blood sample of interest according to manufacturer instructions. These purified blood samples were directly compared to those purified via on-chip extractions.

\subsubsection{DNA quantitation and amplification procedures}

A Picogreen assay quantitation and human $\beta$-globin PCR procedures was adopted from Duarte et $a l^{7}$. In the instance where a multiplexed short tandem repeat (STR) 
amplification was performed, AmpFLSTR $®$ Identifiler ${ }^{\circledR}$ kits (Life Technologies, Frederick, MD) were used according to manufacturer instructions. An ABI PRISM® 310 Genetic Analyzer (Applied Biosystems, Grand Island, NY) was used for obtaining electropherograms post amplification.

\subsection{Results and Discussion}

Controlling fluid flow in a rotationally-driven manner is not a new concept, and this is now recognized as a powerful means for flow control ${ }^{27,30,31}$. We have defined a mechanism for DNA detection, the 'pinwheel effect', based on magnetic particles in a stationary device exposed to rotating magnetic field ${ }^{32,33}$. The inherent 'rotation' involved in centrifugal microfluidics for fluid flow presents a unique opportunity to reverse this configuration by exposing magnetic beads in a moving device to stationary magnets. We show the seamless integration of magnetic bead motion via a rotating extraction device over a stationary magnetic field for the semi-automated dynamic solid phase extraction of DNA.

\subsubsection{Microdevice Design}

A spin-dSPE device shown in Figure 1A was designed with four identical extraction domains, each capable of accepting a separate blood sample loaded directly into the device. The architectural design exploits a number of unique features that become feasible with the print-cut-laminate (PCL) fabrication process we have described previously ${ }^{26}$. This includes architecture with multiple fluidic levels and chamber/channel depths controlled by the number of Pe layers used, and fluid flow control governed by channel geometry and hydrophobic barriers created with printed toner. The features of each layer in the multilayer 


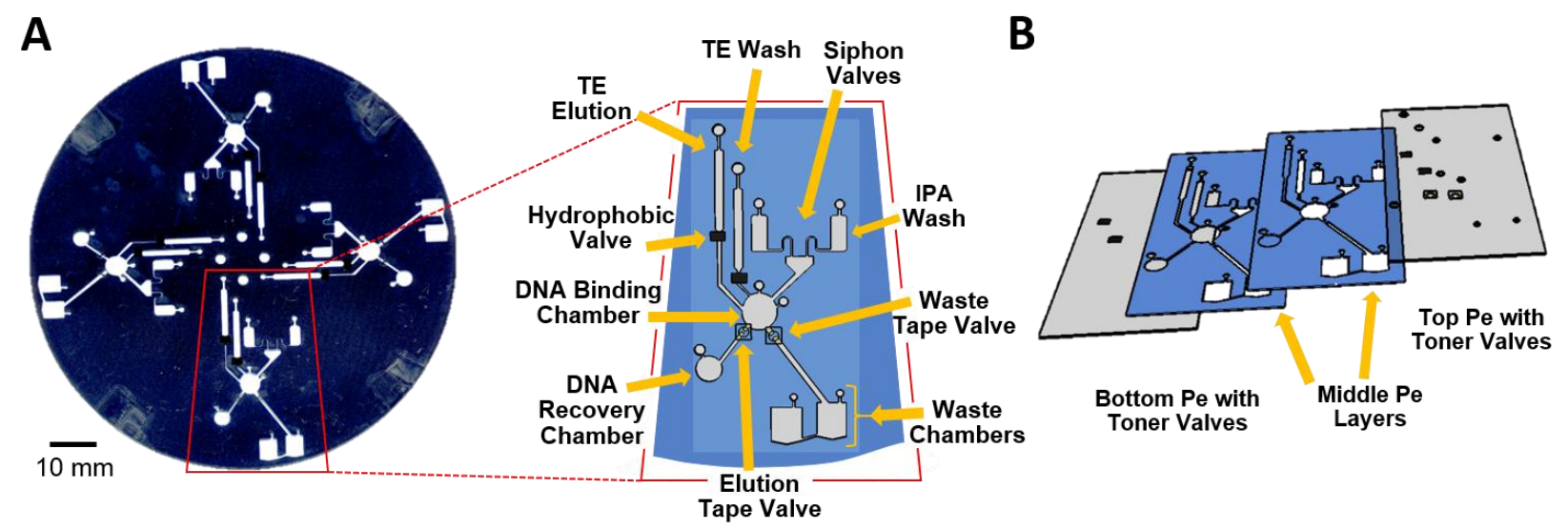

Figure 1: Spin-dSPE Device Design. (A) Front-view illustration of the device with labeled domains for dynamic solid-phase extraction. (B) Illustrated overlay of the different layers of polyethylene $(\mathrm{Pe})$ material to form chambers and connecting channels for spin-dSPE

system, along with the order of assembly, is provided in Figure 1B. The middle Pe layers define the overall architectural dimensions and design for the device while the toner, printed on each middle layer, acts as an adhesive to sandwich these layers together.

The SPE device features needed to carry out the extraction steps described in the introduction are shown in the exploded view in Figure 1A. Here, the central location for DNA binding, washing, and elution chemistry is the DNA binding chamber. Upstream from this are chambers for the reagents needed for washing or elution during the extraction process: isopropyl alcohol (IPA) wash chambers, Tris-EDTA (TE) wash, and TE elution reagents. Downstream from the chamber, the design allows fluid flow in two directions: (i) waste chambers, holding up to $14 \mu \mathrm{L}$ volume, or (ii) a DNA recovery chamber that accommodates up to approximately $7 \mu \mathrm{L}$ total volume of PCR-ready DNA after the completion of an extraction.

It is essential that the wash and elution solutions be retained until sample-bead mixing has occurred and DNA binding is complete. While the toner valves were effective 
for the TE solutions ${ }^{27}$, they were ineffective at retaining IPA solutions above $10 \%$, even with the highest density of toner (100\% grayscale) while the device was static (Fig. 2).

Since the premature introduction of IPA adversely affects DNA binding to silica particles, a combination of

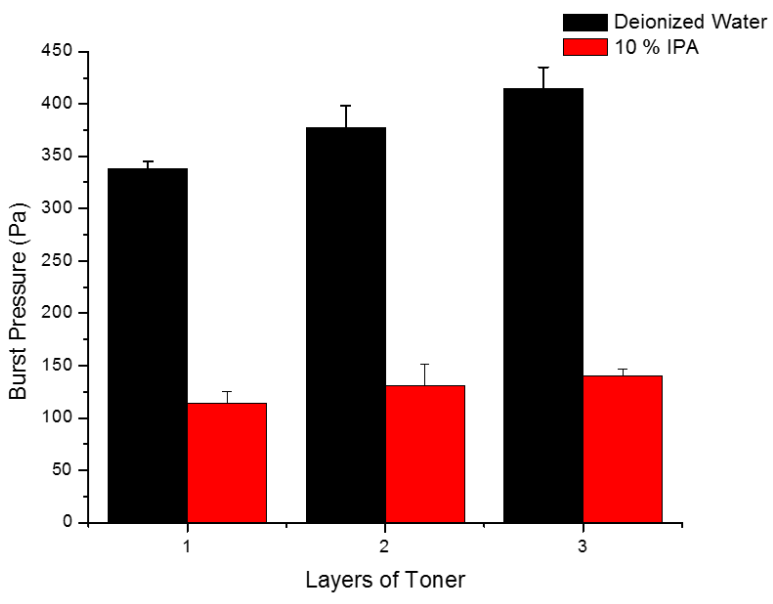

Figure 2: Comparison of the toner valve burst pressure for increased layers of toner between deionized water and a $10 \%$ IPA hydrostatic pressure $^{34}$ and reagent solution ( $90 \%$ deionized water solution) $(n=4)$. separation via gas trapping 31,34 were exploited as a 'valve'. To accomplish this, the IPA chambers were enlarged to have a total volume $0.5 \mu \mathrm{L}$ greater than the volume of solution pumped into the chamber. The chamber enlargement, combined with the removal of air vents, allowed for the introduction of air at the base of the IPA chambers, which prevented the premature release of IPA.

To determine whether there was any structural deformation as a result of the lamination, a novel channel interrogation technique was used to probe the integrity of the microfluidic architecture following fabrication ${ }^{35}$. Briefly, the method involves filling the device with an Allura Red dye solution, scanning on a flatbed scanner, and analyze pixel depth with ImageJ software. We evaluated the extraction chambers as these are the largest features and, thus, had the highest probability of deformation (sagging). The DNA binding chamber, for example, was designed to have a volume of $6.70 \mu \mathrm{L}$, and the post-lamination volume was found to be $6.77 \pm 0.30 \mu \mathrm{L}^{35}$. All dSPE chambers on one device were found to be within $6 \%$ (RSD) of the target volumes, and had average volumes within $2 \%$ error $^{35}$. 
The recovery of DNA following an extraction was critical but was challenging to both contain purified DNA yet provide easy access for post-processing. Since the microdevice rotates during the extraction process, the elution chamber needed to contain the purified sample. Several architectural designs were tested for DNA recovery but were limited by surface tension (preventing fluid from being accessible) or lacked the ability to contain the sample as the device spun. (Fig. 3A). Instead of changing the architecture, a strong adhesive layer was placed around the perimeter of the recovery chamber which could allow a polymer sleeve to be placed on top (Fig. 3B). This worked well to prevent the loss of purified DNA but allowed easy access via puncturing by a syringe or pipette.

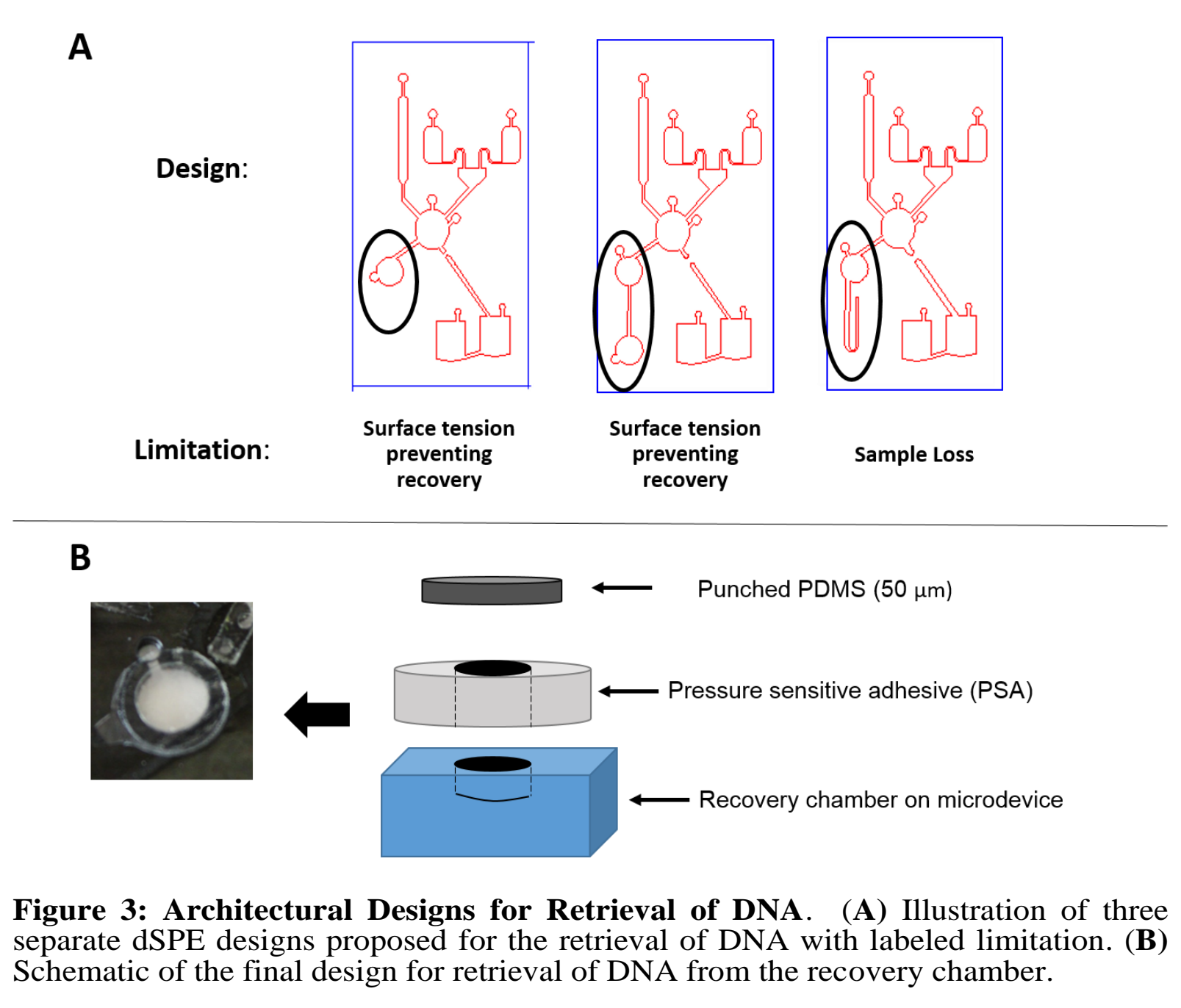




\subsubsection{Fluidic Control}

Although toner valves provide an ideal method for passive fluidic control, their inherent limitations are problematic for dSPE. These include: (1) a maximum burst pressure equivalent to spinning at $9.17 \mathrm{~Hz}^{27}$; (2) a burst pressure accuracy of $\pm 0.5 \mathrm{~Hz}$, due to variation in toner printing (not to mechanical variation in spin speed which was $\pm 1.67 \times 10^{-3} \mathrm{~Hz}$ ) (Fig. 4); (3)
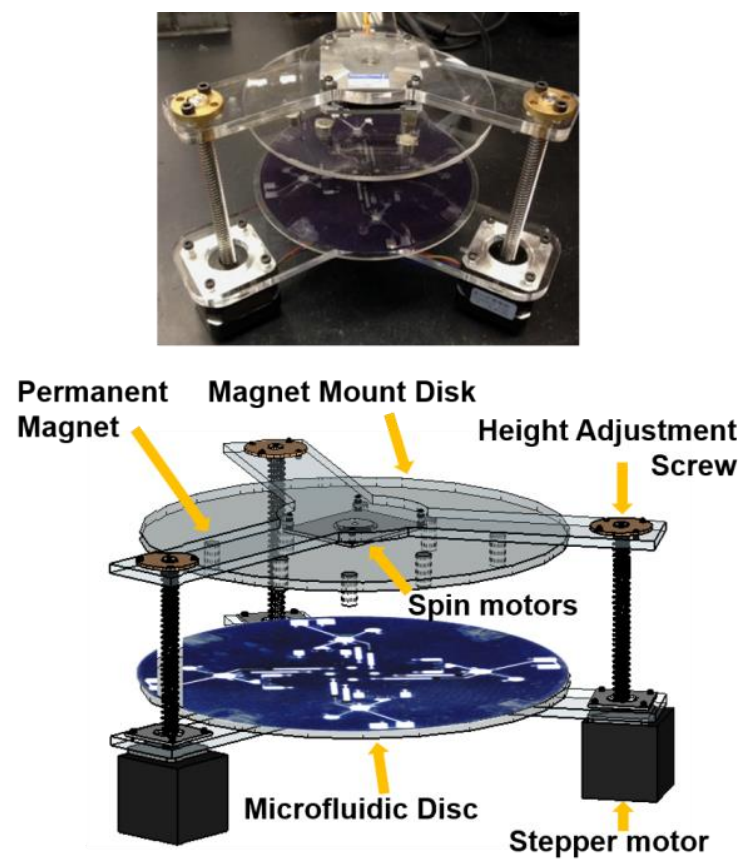

Figure 4: Setup for dSPE rotationallydriven platform with 3D labeled schematic.

incompatibility with IPA; and (4) one-time actuation. Hence, an alternative but complementary valving approach to toner valves was explored. Bifunctional valves (actuatable in both the 'normally-open' and 'normally-closed state) were sought to direct IPA to the waste during the extraction process. These are labelled in the exploded view in Figure 1A (expanded figure) as 'tape valves', and emanate from the work of Lounsbury et $a l .{ }^{29}$ who utilized an adhesive membrane and a screw to isolate fluid in a microchamber during heating ${ }^{29}$.

We have evolved the Lounsbury et al. valve and exploited the adhesive properties to create an adhesive tape valve (ATV) (Fig. 5A). In configuration, this valve is similar to that described by Grover et al. ${ }^{36}$, but with two distinct differences. First, the elastomeric membrane used in the Grover et al. valve was replaced here with a non-elastomeric tape 
capable of 'sealing' on a barrier between two channels. Secondly, where the Grover et al. valve was 'normally-open' until actuated, the ATV can be configured as 'normally-open' or 'normally-closed' in the resting state. Using both a PSA and a double-sided adhesive (DSA), the functionality of the ATV in Figure 5B, is achievable. The 'normally open' ATV allows fluid to pass until manual pressure is applied to force contact of the PSA with the valve seat, sealing off the connection between channels. Fluid driven with adequate centrifugal force generate enough
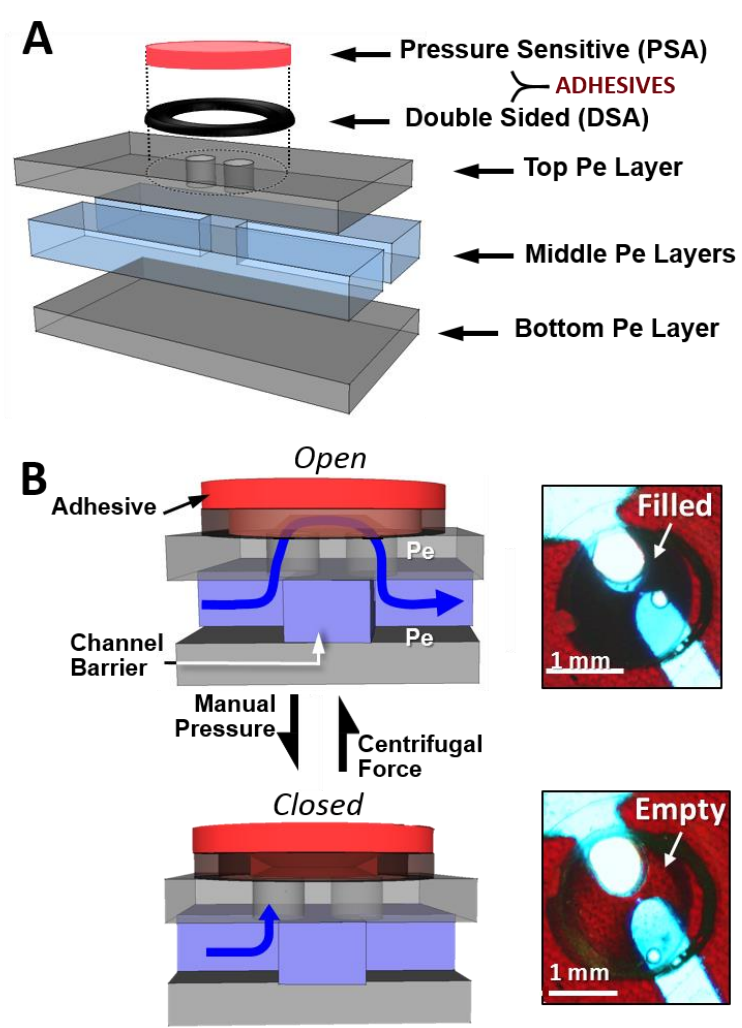

Figure 5: Schematic for Physical Tape Valves on dSPE Microfluidic Device. (A) 3D Illustration of a tape valve used for fluidic control. (B) Side view tape valve open and closed orientations with accompanying pictures of valve in labeled open or closed state.

pressure to delaminate the PSA from the channel barrier and re-open the valve.

Each extraction requires the use of a 'normally open' waste ATV and a 'normally closed' elution ATV (Fig. 1A) allowing all wash reagents to pass through to the waste chambers. After all wash steps were completed, a blunt object was used to close the waste ATV, where it remained closed for the duration of the extraction. Following DNA elution from the silica surface, hydraulic pressure (generated by higher spin speeds) opened the elution ATV, and allowed for mobilization of the PCR-ready DNA from the binding chamber to the DNA recovery chamber. 


\section{A}

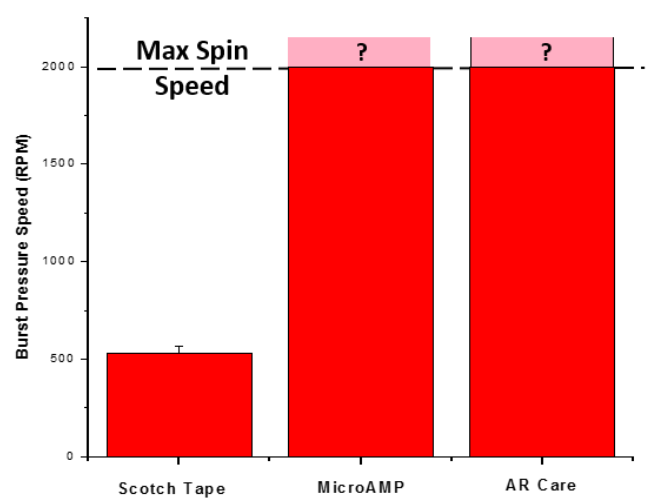

B

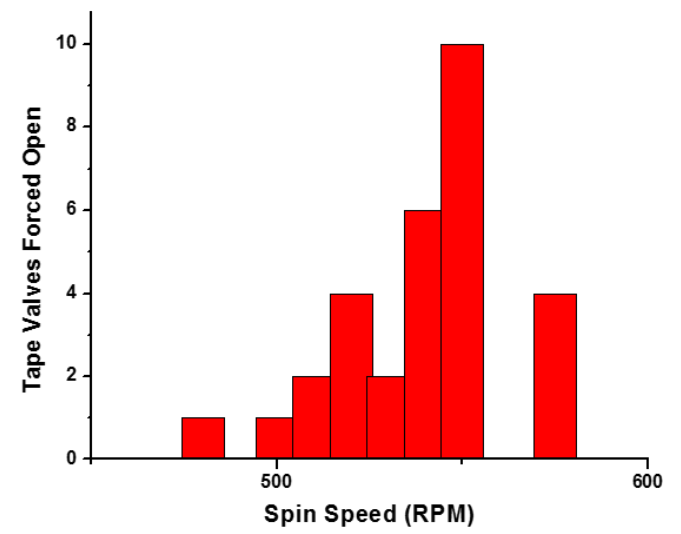

Figure 6: Performance of Tape Valves on dSPE Microfluidic Device. (A) Comparison of the burst pressure for adhesives tested. (B) Histogram correlating the spin speed to the number of tape valves forced open from a closed position.

Three important characteristics of ATVs were considered for the extraction device: 1) the ability to remain closed at low spin speeds;2) the ability to generate enough hydraulic pressure to burst the valve at high spin speeds; and 3) the reagent compatibility of the valve. Both the low and high spin speed functionality depend on 'bond strength' of the ATV, which is a primary parameter determining the burst frequency of the valve. Other valve parameters included the surface area of the channel barrier ${ }^{37,38}$ and the radial distance from the center ${ }^{38}$.

We explored several adhesives as potential candidates to function as part of the ATV based on bond strength, availability, ease of use, and biocompatibility. We compared the bond strength of Scotch® tape, Microamp adhesive tape, and ARcare to Pe film, using the burst frequency as the metric, i.e., rotation speed associated with fluid breakthrough within $10 \mathrm{sec}$ of reaching the targeted speed. The results in $0.83 \mathrm{~Hz}$ increments are given in Figure 6A. The Microamp and ARcare adhesives reproducibly resisted delamination at spin speeds up to $33.33 \mathrm{~Hz}(\mathrm{n}=10)$. In contrast, the $S \operatorname{cotch}{ }^{\circledR}$ tape resisted delamination at spin frequencies up to $\sim 8.33 \mathrm{~Hz}(8.83 \pm 0.58 \mathrm{~Hz} ; \mathrm{n}=10)$, which was ideal given the functional 


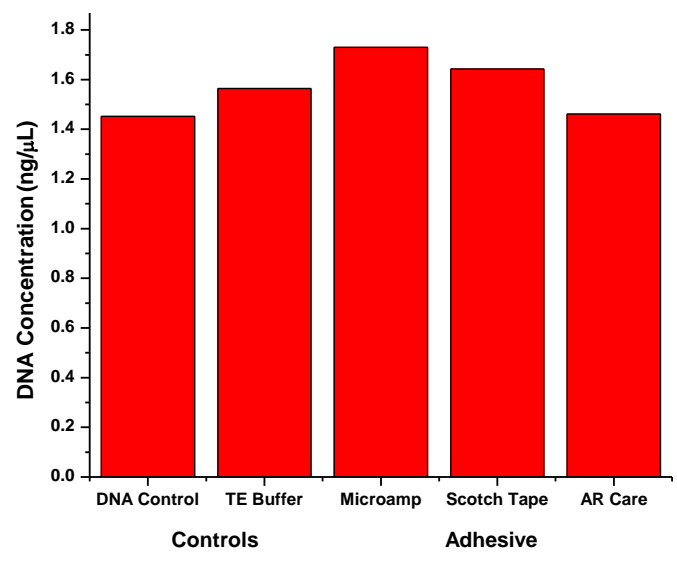

Figure 7: qPCR analysis of amplifiable DNA with various adhesive tapes added to a DNA control sample. spin frequency range of the system (0-33.33 Hz). As shown in Figure 7, none of the adhesives were found to affect downstream PCR amplification of DNA (validated via qPCR), hence, Scotch ${ }^{\circledR}$ tape was selected as the best adhesive for all future ATVs.

Having defined the appropriate substrate and established functionality criteria for the ATV, we sought to evaluate the

reproducibility of the ATV opening. This was accomplished using spin devices with replicate (4 per device) 'closed state' ATVs where fluid was loaded into the device and rotational frequency increased in $0.17 \mathrm{~Hz}$ increments; the number of valves that opened within $5 \mathrm{sec}$ of reaching the desired spin speed was evaluated. As shown in Figure 6B, 29 of the 30 ATVs opened at $8.33 \mathrm{~Hz}$ or greater, indicating that a multistep protocol could be carried out at sub-9 Hz speeds without bursting the ATV. The single failed valve (1/30) resulted from inadequate manual sealing of the adhesive.

\subsubsection{Optimization of the Spin Frequency Protocol for dSPE of DNA}

Sequence-specific addition of reagents is necessary for successful dSPE, therefore, it was pertinent to optimize the following steps outlined in Figure 8: (i) initial binding of DNA to silica particles, following sample addition (ii) washing of the bead mixture with IPA, (iii) washing of the bead mixture with TE buffer, (iv) introduction of TE elution for desorption of DNA from the silica particles, and finally, (v) transfer of the purified DNA 
to a recovery chamber. If any of these steps failed, it would compromise the nature of the DNA and result in unsuccessful recovery of the DNA and/or poor PCR amplification. Prior to initiating the device spin protocol, all reagents were loaded into the appropriate chambers (see 'prior to spin'; Fig. 8). A rotational speed of $5 \mathrm{~Hz}$ mobilized the IPA without overcoming the burst frequency of any of the TE buffer toner valves, therefore, allowing the IPA to remove the $\mathrm{GuHCl}$, and any weakly adsorbed components while leaving the DNA tightly bound to the particles ('Step 1'; Fig. 8). Spinning at $6.67 \mathrm{~Hz}$ released the TE
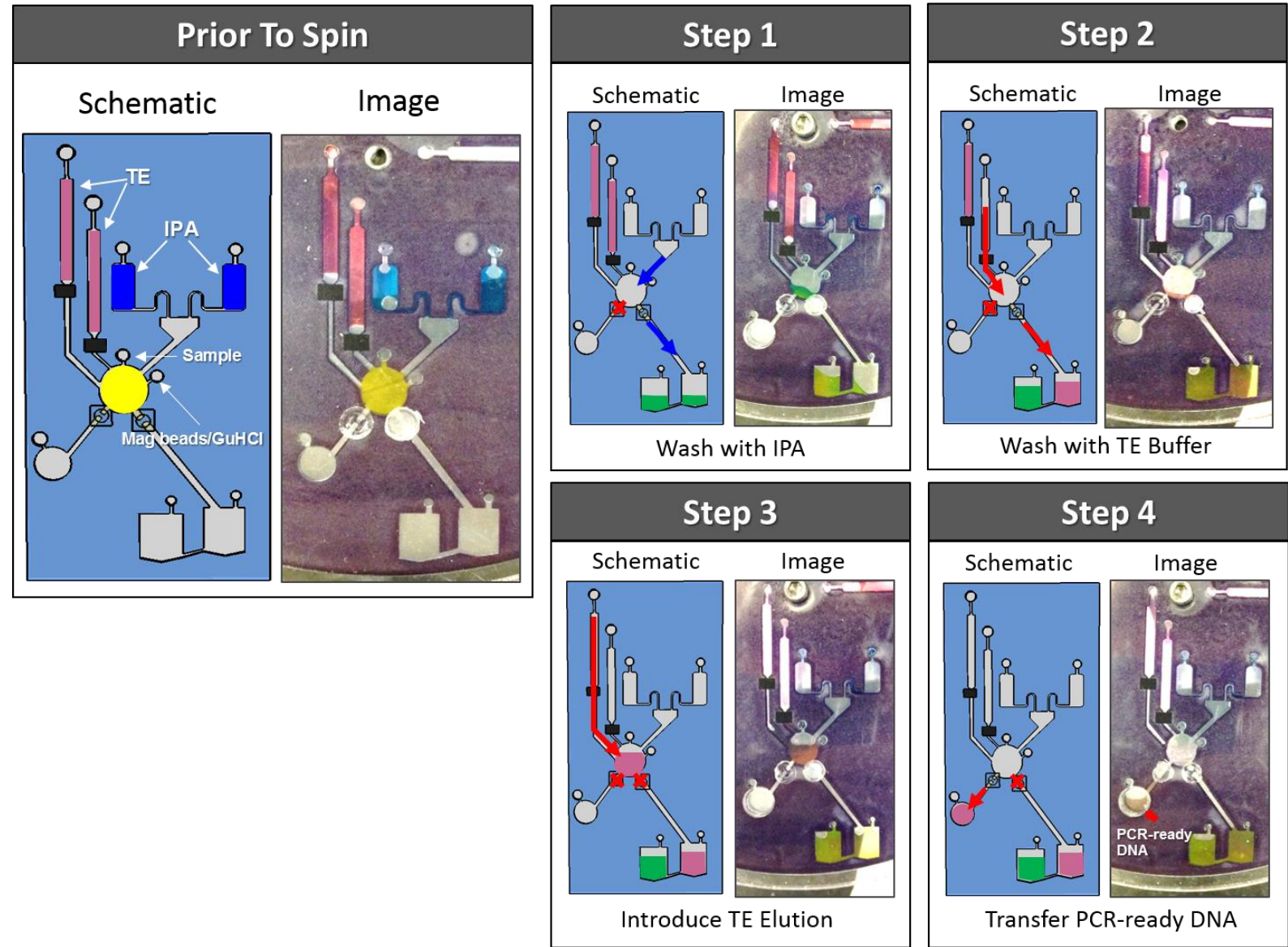

Figure 8: Optimized method for the extraction of DNA from raw samples. Illustration of loaded spin-dSPE device, ready for extraction followed by representation of the steps for on-chip dSPE. Step 1: IPA wash $(5 \mathrm{~Hz}, 120$ seconds) following initial mixing to bind DNA. Step 2: TE buffer wash $(6.67 \mathrm{~Hz}, 10$ seconds). Step 3: Load elution buffer $(11.67 \mathrm{~Hz}, 5$ seconds) and mix to elute DNA. Step 4: Transfer elution buffer to separate chamber for post processing (13.33 Hz, 5 seconds). 
wash buffer without affecting the elution chamber ('Step 2'; Fig. 8), displacing any residual IPA and $\mathrm{GuHCl}$ that could be inhibitory to DNA amplification. Following this step, the waste ATV was manually closed.

Since toner valves (100\% grayscale) break at $9.5 \pm 0.5 \mathrm{~Hz}$, the device was rapidly accelerated to a speed that exceeded this threshold $(1.67 \mathrm{~Hz}$ in this case) to ensure the opening of all toner valves. Note that the ATV breaks at $8.83 \mathrm{~Hz}$, and while it may seem problematic to transiently exceed this spin speed, fluid has not reached the downstream architecture at this point and, thus, is not a problem ('Step 3'; Fig. 8). Once fluid had reached the ATVs, the TE buffer hydrostatic pressure was below that of the burst frequency of the tape valves, therefore, the closed ATV prevented the TE from leaving the binding chamber.

After the DNA was desorbed from the magnetic particles by mixing in TE buffer, a rapid counter-clockwise acceleration $\left(1.5 \mathrm{~Hz} \mathrm{sec}^{-1}\right)$ forced the elution ATV open, allowing transfer of the PCR-ready DNA to the DNA recovery chamber ('Step 4'; Fig. 8). The overall success rate for 40 DNA extractions on 10 microdevices was $77 \%$, with a $100 \%$ success in IPA containment. We are aware that a $77 \%$ success is not acceptable for immediate adoption in any field, however, the advances described here are significant for the following reasons: 1) minimized external hardware for fluidic control, i.e., no syringe pumps or complex valving hardware ${ }^{15,16}$, and 2) operation on a low-cost centrifugal system made from COTS components. Further improvements are currently underway with a focus on burst pressure variability of valves $(10 \%$ for the elution buffer toner valve and $7 \%$ for the ATVs) and the integration of sample lysis. 


\subsubsection{Magnetic Field-induced Mixing}

Rotating magnetic field mixing of magnetic silica particles and DNA is not new and has been exploited over the last several years ${ }^{32,33,39-41}$. The strong affinity of DNA for silica surfaces in a chaotropic salt is attributed to shielded intermolecular electrostatic forces, dehydration of DNA and silica surfaces, and a hydrogen bond formation between DNA and silica particles ${ }^{32,40}$. While the rotating magnetic field approach is valuable, it isn't compatible with the PeT device, as channel depths of $1.5 \mathrm{~mm}$ cannot be achieved and an equivalent magnetic field provided low extraction efficiencies $(<25 \%)$. Therefore, we
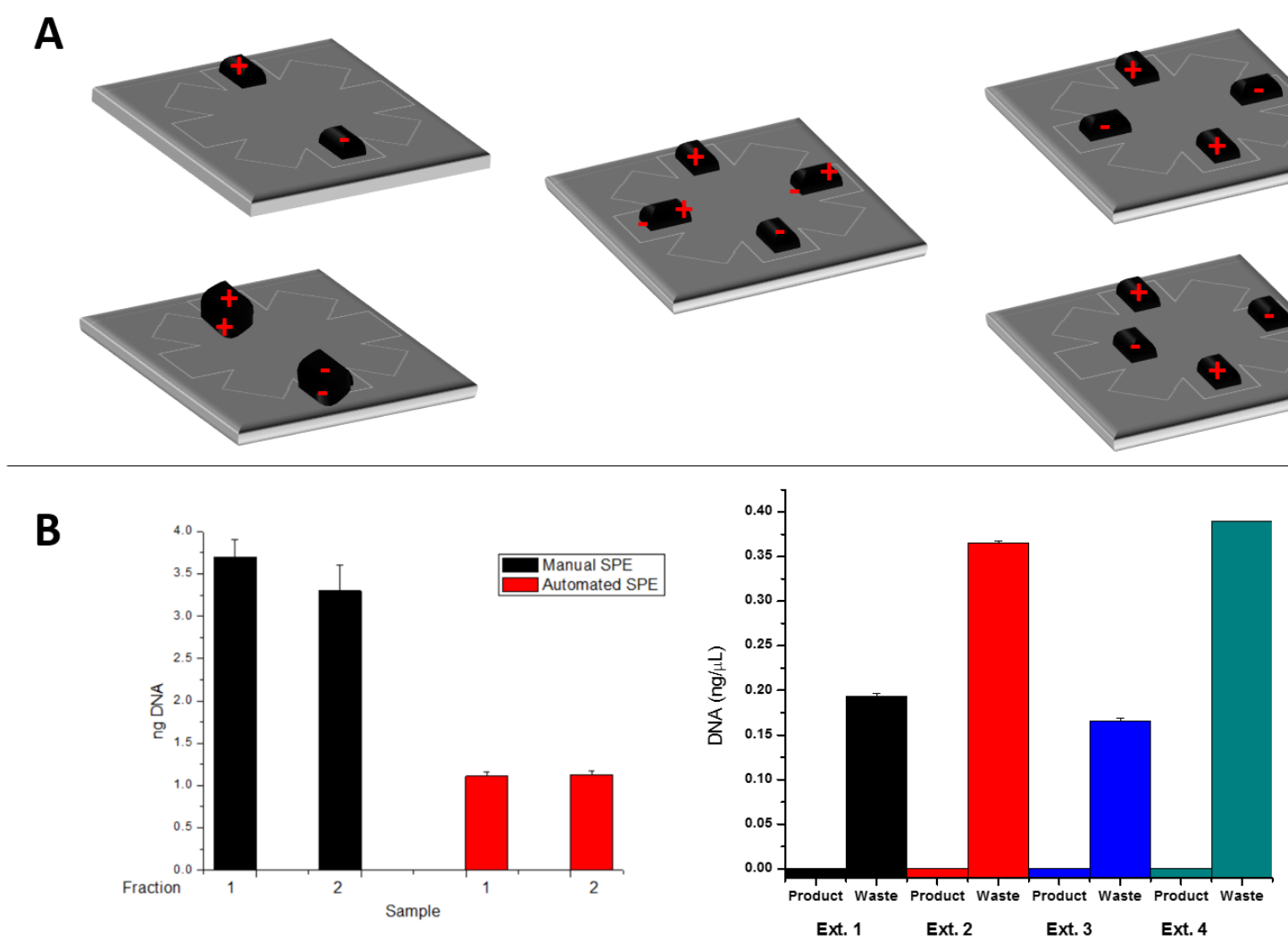

Figure 9: Optimizing Automated Mixing Strategy. (A) Schematic of selected magnetic arrays tested for dSPE (magnetic field oriented towards the microdevice). (B) Analysis of automated mixing compared to manual mixing (left) and the distribution between the amount of DNA present in the recovery chamber (product) versus the waste (right). 
worked towards obtaining a sufficient magnetic field with three key properties in mind: position of the magnets, magnetic mixing distance, and mixing time.

Various magnetic arrays were tested to observe the mixing efficiency of the beads and DNA. Figure 9A shows some of the magnetic fields which were tested for dSPE. Unfortunately, none of these magnetic fields provided an extraction efficiency above $20 \%$, which is not acceptable for on-chip DNA extraction. The mixing efficiency for manual mixing was significantly higher than when the mixing was automated, as shown in Figure 9B. In some cases, the DNA was found only in the waste, which was indicative of problems binding the DNA to the beads. As a higher extraction efficiency was needed for DNA extraction, an alternative mixing scheme was explored.

The optimal mixing strategy employed used two magnets with opposing polarities facing the sample device (Fig. 10). When the sample device was moved bi-directionally through $43^{\circ}$, attraction to the positive magnetic pole mobilized the particles to the lower left quadrant of the DNA chamber (position "A";

Fig. 10). When the device was rotated in the opposite direction, attraction to the positive magnetic pole mobilized the particles to the top right quadrant of the DNA chamber (position "B"; Fig. 10). Repeating this bidirectional motion between the two magnetic poles at a

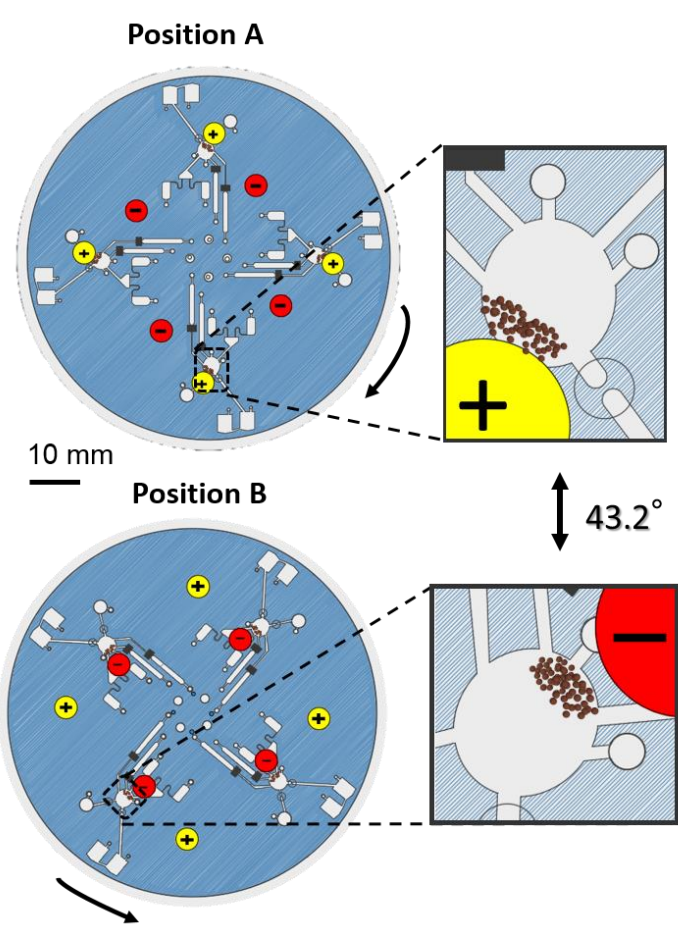

Figure 10: Top-view Illustration of Bi-directional sway mixing of magnetic field from between position $A$ and $B$ at a $10 \mathrm{~mm}$ height differential from the sample device. 
frequency of $0.736 \mathrm{~Hz}$ led to a "sweeping" effect with the beads. This allowed the beads maximum opportunity to interact with the DNA.

The optimal vertical distance between the magnetics and sample device was determined empirically for maximum bead movement, and found to be in the 7-10 $\mathrm{mm}$ range. At this distance, the beads responded rapidly to the external magnetic field, mobilizing from one side of the chamber to the other within $5 \mathrm{sec}$. Outside of this range, bead movement was sluggish and required substantially longer exposure times (20-30 sec) to traverse the width of the chamber. Given this, a height of $10 \mathrm{~mm}$ was used for all experiments.

The extraction efficiency of DNA from whole blood with known WBC counts (99\% of the DNA in whole blood originates from WBC DNA) was used as a metric for determining an optimal mixing time. Studies on genomic DNA extraction from lysed blood suggest a minimum mixing time of $240 \mathrm{sec}$ as a starting point using an alternative high speed mixing strategy ( +9 to $-9 \mathrm{~Hz}$ speed with a $60 \mathrm{~Hz} \sec ^{-1}$ acceleration $)^{21}$. Mimicking this mixing approach was not possible because the speeds required are larger than the burst frequency of the ATVs. Mixing via bidirectional sweeping was explored at $240 \mathrm{sec}$, but did not provide sufficient extraction efficiencies $(24.4 \% ; \mathrm{n}=3)$. Increasing the mixing time to $500 \mathrm{sec}$ alternating between the poles every $5 \mathrm{sec}$ improved the extraction efficiency dramatically, with an average of $44 \pm 4.4 \%$ ( $n=4)$; consequently, this was set as the mixing time for further experiments. 


\subsubsection{Validation of On-chip Dynamic Solid-phase}

\section{Extraction}

With an optimized mixing method and spin protocol, it was imperative to compare the spindSPE device to that of current technologies. To do this, the extraction efficiencies with spin-dSPE were compared with: i) on-chip centrifugal extraction using a manual magnet manipulation technique by Duarte et al. ${ }^{10}$, ii) dSPE in a PeT channel using manual pipetting and mixing steps ${ }^{7}$,

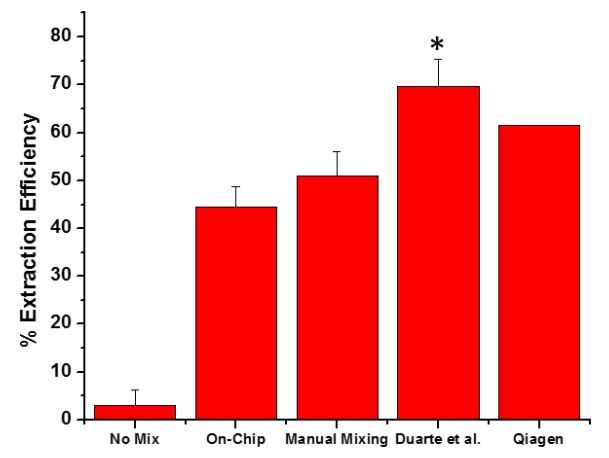

Figure 11: Comparison of on-chip dSPE chip extractions to other extraction methods $(n=4)$ : dSPE via manual mixing and no mixing, a previously described dSPE method $^{12}$, and a conventional Qiagen extraction. Note: * Indicates multiple elutions from the extraction

and iii) conventional extraction (Fig. 11). Other extraction parameters for these methods are summarized and compared in Table 1.

On a single device, the spin-dSPE device yielded an average extraction efficiency of $44.5 \pm 4.4 \%(n=4)$. Although this is substantially lower than the $69.7 \pm 5.7 \%$ reported by Duarte et al. ${ }^{10}$, the initial elution provided a $34.2 \%$ extraction efficiency (of the total 69.7\%), which is lower than the efficiency obtained with the spin-dSPE method. In

Table 1: Summary of Evaluated Extraction Methods

\begin{tabular}{|c|c|c|c|c|c|c|c|c|c|c|}
\hline \multirow{2}{*}{$\begin{array}{l}\text { Extraction } \\
\text { method }\end{array}$} & \multirow{2}{*}{$\begin{array}{c}\text { Sample } \\
\text { Volume } \\
(\mu \mathrm{L})\end{array}$} & \multirow{2}{*}{$\begin{array}{c}\text { Elution } \\
\text { Volume } \\
(\mu \mathrm{L})\end{array}$} & \multirow{2}{*}{$\begin{array}{c}\text { Total } \\
\text { Extraction } \\
\text { Time (min) }\end{array}$} & \multirow{2}{*}{$\begin{array}{c}\text { Manual } \\
\text { Manipulation } \\
\text { Time (min) }\end{array}$} & \multicolumn{6}{|c|}{ Automated steps* } \\
\hline & & & & & $\begin{array}{c}\text { DNA } \\
\text { Binding }\end{array}$ & $\begin{array}{c}\text { IPA } \\
\text { Wash }\end{array}$ & $\begin{array}{c}\text { TE } \\
\text { Wash }\end{array}$ & $\begin{array}{c}\mathrm{TE} \\
\text { Elution }\end{array}$ & $\begin{array}{c}\text { DNA } \\
\text { Desorption }\end{array}$ & $\begin{array}{l}\text { DNA Transfer } \\
\text { for Recovery }\end{array}$ \\
\hline Conventional & 2 & 50 & $25-30$ & $20-25$ & & & & & & \\
\hline $\begin{array}{c}\text { PeT channel } \\
\text { dSPE }\end{array}$ & 2 & 2 & 30 & 30 & & & & & & \\
\hline $\begin{array}{l}\text { Manual mixing } \\
\text { Spin - dSPE }\end{array}$ & 2 & 6 & 25 & 16 & & & & & & \\
\hline Spin - dSPE & 2 & 6 & 20 & 0.5 & & & & & & \\
\hline
\end{tabular}

* Green indicates automation, red indicates manual manipulation. 
addition, there was no significant difference in the average extraction efficiency between a manual mixing method and our on-chip bead mixing method, as shown by overlapping error bars. While DNA extraction spin-dSPE device provided lower extraction efficiencies than that obtained with commercial column extraction systems (69\% extraction efficiency) data discussed in the next section shows that the ultimate metric - PCR-amplifiable DNA was demonstrated.

\subsubsection{PCR Amplification of Spin-dSPE Purified DNA}

The processes downstream of DNA extraction, specifically, PCR, can be sensitive to changes in the integrity of the DNA (intact vs. degraded) and small molecule inhibitors of the polymerase. To verify PCR-readiness of the DNA yielded from on-chip extractions, two PCR amplification assays were carried out: a singleplex amplification of a sequence from the $\beta$-globin gene, and a multiplexed amplification of 16 target locations in the DNA. For the $\beta$-globin sequence, amplification was carried out with equal masses of pre-purified DNA and DNA purified on the spin-dSPE system. The electropherogram in Figure 12A shows the very clean amplification (i.e., no non-specific amplification) of the $389 \mathrm{bp}$ amplicon from the $\beta$-globin gene, and was exemplary of the type singleplex amplifications that were observed with use of pre-purified DNA.

Carrying out the amplification of multiple targets with a single thermocycling protocol is enhanced with DNA of increased purity and devoid of PCR inhibitors, such as GuHCl and IPA, as they inhibit the polymerase activity ${ }^{5}$. The multiplexed assay used for this was a forensic short tandem repeat (STR) amplification for human identification ${ }^{5}$. This 
A
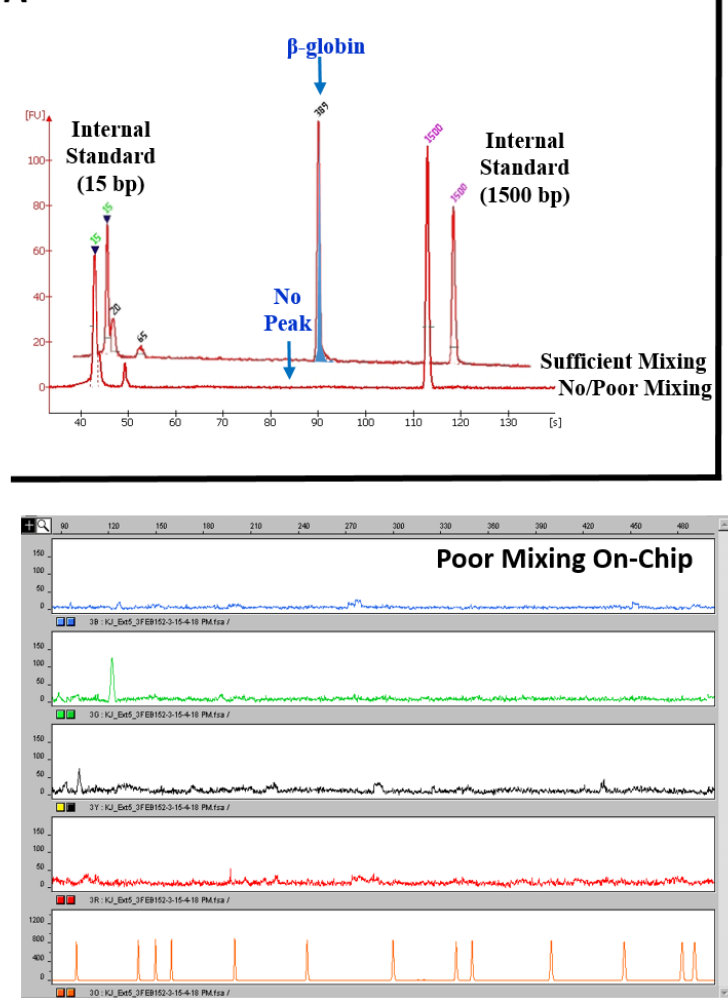

Figure 12: Post-extraction Amplification of On-Chip DNA. (A) Overlayed electropherograms comparing PCR amplification of $\beta$-globin from an on-chip extraction with and without sufficient mixing. (B) Comparison of a well and poor mixed on-chip extraction to a conventional column extraction.

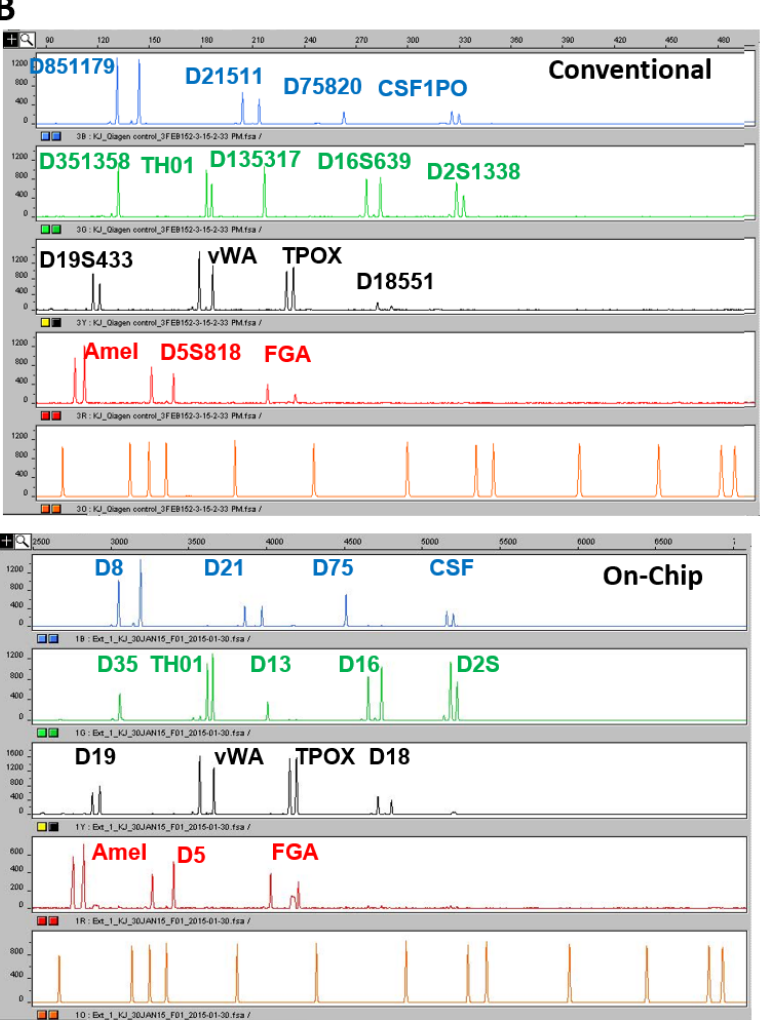

involves DNA amplification at 16 different location in the genome and will amplify between 16 - 32 separate fragments. Successful amplification of all amplicons can be seen in Figure 12B where the upper right panel shows the resultant amplification using DNA purified by conventional means. The same amplification using the spin-dSPE purified DNA (lower right panel) shows that the 'on-chip' purification compares favourably with the conventional extraction method. While there may be concern that no negative control is shown (e.g., contaminate DNA from the user or other sources), it is, in fact, inherent in the experiment that has been done. Since a single-source male DNA template is used here, contamination from the user would be apparent in the form of; (1) a female signature for amelogenin; (2) the presence of additional amplicons at each loci, and (3) variation between 
the conventional extraction and a spin-dSPE extraction. The importance of the mixing steps, driven by the interaction with a static external magnetic field, becomes clear when there is 'poor mixing on-chip' (lower left panel), where a failed amplification resulted from either poor extraction of sample DNA or ineffective removal of inhibitors. Advantages of the spin-dSPE system include a smaller input sample, resultant purified DNA concentrated in a smaller volume, and the potential for cost-per-extraction to be reduced $10-$ fold $^{42}$.

This is the first demonstration of a simple centrifugal device that provides PCRready DNA from $<1 \mu \mathrm{L}$ (specifically, $0.6 \mu \mathrm{L}$ ) of blood. Many of the methods describing DNA extraction on a centrifugal device do not purify DNA and involved large sample volumes $(>100 \mu \mathrm{L})$ of whole blood ${ }^{15,21,23,24}$ that are rarely encountered in forensics. In addition to describing a 100-fold reduction in sample volume relative to previous reports, we also demonstrate an extraction system that accommodates a 2-4 fold increase in the total samples on a single device ${ }^{15,16,21-24}$.

\subsection{Conclusions}

Successful semi-automated DNA extraction has been demonstrated on a disposable PeT centrifugal device using a novel in-house spinning platform and unique mixing via an external magnetic field. For the first time, we show the utility of adhesive tape valves, capable of functioning in both the 'normally open' and 'normally closed' states, and demonstrate their integration with passive flow control on a PeT device. The spin-dSPE protocol yields PCR-ready DNA in a manner that allows for the simultaneous extraction of four samples. The integrity of the extracted DNA was confirmed by the successful amplification in simple and complex implication schemes. 
Perhaps more important are the attributes of the semi-automated DNA extraction system that make it unique. Unlike other centrifugal microfluidic systems reported in the literature with elegant in design and function ${ }^{18,21}$, the spin-dSPE system we describe here uniquely provides extracted DNA in a pure form completely free of PCR inhibitors. While other systems have reported the successful purification of DNA from large volume (100 4 l) blood samples ${ }^{22,24}$, samples routinely encountered in the forensic sector involve substantially smaller volumes, and it is here that the spin-dSPE system we describe is effective. To this point, it is capable of extracting DNA from small, precious samples where the extracted DNA must be eluted in small volumes for direct PCR amplification. However, in addition, provides an extraction efficiency of $44 \pm 4.4 \%$ from human genomic DNA, an improvement over the $31 \pm 10 \%$ reported for automated bacterial DNA extraction ${ }^{24}$. Moreover, the system described here elutes DNA in volumes that are 10-fold lower than automated centrifugal systems ${ }^{22,24}$ and 100-fold lower than commercial systems without compromising extraction efficiency. This is particularly advantageous with most forensic applications.

Future efforts are focused on automating the spin-dSPE process in a hands-free system with comprehensive passive valving. This, along with modifications that: 1) include the sample lysis step, and 2) will accommodate other sample types (e.g., buccal swabs), will be critical to defining a functional system for a broad range of genomic analyses.

\subsection{References}

1. J. B. B. K. R. Jackson, M. Meija, D. L. Mills, D. M. Haverstick, K. E. Olson, R. Aranda, G. T. Garner, E. Carrilho and J. P. Landers, Analytica Chimica Acta, Submitted. 
2. S. Park, Y. Zhang, S. Lin, T.-H. Wang and S. Yang, Biotechnology Advances, 2011, 29, 830-839.

3. W. A. Al-Soud and P. Rådström, Journal of Clinical Microbiology, 2001, 39, 485-493.

4. B. Rittich and A. Spanova, Journal of Separation Science, 2013, 36, 2472-2485.

5. J. Butler, Advanced Topics in Forensic DNA Typing, Academic Press, 2011.

6. C. W. Price, D. C. Leslie and J. P. Landers, Lab on a Chip, 2009, 9, 2484-2494.

7. G. R. M. Duarte, C. W. Price, J. L. Littlewood, D. M. Haverstick, J. P. Ferrance, E. Carrilho and J. P. Landers, Analyst, 2010, 135, 531-537.

8. J. Wu, R. Kodzius, W. Cao and W. Wen, Microchim Acta, 2014, 181, 1611-1631.

9. K. M. Horsman, J. M. Bienvenue, K. R. Blasier and J. P. Landers, Journal of Forensic Sciences, 2007, 52, 784-799.

10. G. R. M. Duarte, C. W. Price, B. H. Augustine, E. Carrilho and J. P. Landers, Analytical Chemistry, $2011, \mathbf{8 3}, 5182-5189$.

11. F. Ahmad and S. A. Hashsham, Analytica Chimica Acta, 2012, 733, 1-15.

12. A. Manz, D. J. Harrison, E. M. J. Verpoorte, J. C. Fettinger, A. Paulus, H. Ludi and H. M. Widmer, Journal of Chromatography, 1992, 593, 253-258.

13. W. K. T. Coltro, D. P. de Jesus, J. A. F. da Silva, C. L. do Lago and E. Carrilho, Electrophoresis, 2010, 31, 2487-2498.

14. D. Mark, S. Haeberle, G. Roth, F. Von Stetten and R. Zengerle, in Microfluidics Based Microsystems, eds. S. Kakaç, B. Kosoy, D. Li and A. Pramuanjaroenkij, Springer Netherlands, 2010, DOI: 10.1007/978-90-481-9029-4_17, ch. 17, pp. 305-376.

15. S. Azimi, G. Nixon, J. Ahern and W. Balachandran, Microfluidics and Nanofluidics, 2011, 11, 157165.

16. W. P. Gan, B. Zhuang, P. F. Zhang, J. P. Han, C. X. Li and P. Liu, Lab on a Chip, 2014, 14, 37193728.

17. J. Ducree, S. Haeberle, S. Lutz, S. Pausch, F. von Stetten and R. Zengerle, Journal of Micromechanics and Microengineering, 2007, 17, S103-S115. 
18. R. Gorkin, J. Park, J. Siegrist, M. Amasia, B. S. Lee, J. M. Park, J. Kim, H. Kim, M. Madou and Y. K. Cho, Lab on a Chip, 2010, 10, 1758-1773.

19. M. Vázquez, D. Brabazon, F. Shang, J. O. Omamogho, J. D. Glennon and B. Paull, TrAC Trends in Analytical Chemistry, 2011, 30, 1575-1586.

20. Z. J. Madou M, Jia G, Kido H, Kim J, Kim N., Annu Rev Biomed Eng., 2006, 8, 601-628.

21. Y. K. Cho, J. G. Lee, J. M. Park, B. S. Lee, Y. Lee and C. Ko, Lab on a Chip, 2007, 7, 565-573.

22. M. Karle, J. Miwa, G. Czilwik, V. Auwarter, G. Roth, R. Zengerle and F. von Stetten, Lab on a Chip, 2010, 10, 3284-3290.

23. J. Siegrist, R. Gorkin, M. Bastien, G. Stewart, R. Peytavi, H. Kido, M. Bergeron and M. Madou, Lab on a Chip, 2010, 10, 363-371.

24. O. Strohmeier, A. Emperle, G. Roth, D. Mark, R. Zengerle and F. von Stetten, Lab on a Chip, 2013, 13, 146-155.

25. C. Lucio do Lago, H. D. Torres da Silva, C. A. Neves, J. G. Alves Brito-Neto and J. A. Fracassi da Silva, Analytical Chemistry, 2003, 75, 3853-3858.

26. B. L. Thompson, Y. Ouyang, G. R. M. Duarte, E. Carrilho, S. T. Krauss and J. P. Landers, Nat. Protocols, 2015, 10, 875-886.

27. Y. W. Ouyang, S. B. Wang, J. Y. Li, P. S. Riehl, M. Begley and J. P. Landers, Lab on a Chip, 2013, 13, 1762-1771.

28. J. Kim, H. Kido, R. H. Rangel and M. J. Madou, Sensors and Actuators B: Chemical, 2008, 128, 613-621.

29. J. A. Lounsbury, A. Karlsson, D. C. Miranian, S. M. Cronk, D. A. Nelson, J. Li, D. M. Haverstick, P. Kinnon, D. J. Saul and J. P. Landers, Lab on a Chip, 2013, 13, 1384-1393.

30. H. Cho, H.-Y. Kim, J. Y. Kang and T. S. Kim, Journal of Colloid and Interface Science, 2007, 306, 379-385.

31. R. Gorkin Iii, C. E. Nwankire, J. Gaughran, X. Zhang, G. G. Donohoe, M. Rook, R. O'Kennedy and J. Ducree, Lab on a Chip, 2012, 12, 2894-2902. 
32. D. C. Leslie, J. Li, B. C. Strachan, M. R. Begley, D. Finkler, L. A. L. Bazydlo, N. S. Barker, D. M. Haverstick, M. Utz and J. P. Landers, Journal of the American Chemical Society, 2012, 134, 56895696.

33. D. A. Nelson, B. C. Strachan, H. S. Sloane, J. Li and J. P. Landers, Analytica Chimica Acta, 2014, 819, 34-41.

34. D. J. Kinahan, S. M. Kearney, N. Dimov, M. T. Glynn and J. Ducree, Lab on a Chip, 2014, 14, 2249-2258.

35. Q. L. K. Xu, K. Jackson, and J. P. Landers, Accepted; Nature Scientific Report, 2015.

36. W. H. Grover, A. M. Skelley, C. N. Liu, E. T. Lagally and R. A. Mathies, Sensors and Actuators B-Chemical, 2003, 89, 315-323.

37. B. Mosadegh, C.-H. Kuo, Y.-C. Tung, Y.-s. Torisawa, T. Bersano-Begey, H. Tavana and S. Takayama, Nat Phys, 2010, 6, 433-437.

38. H. Hwang, H.-H. Kim and Y.-K. Cho, Lab on a Chip, 2011, 11, 1434-1436.

39. B. C. Strachan, H. S. Sloane, J. C. Lee, D. C. Leslie and J. P. Landers, Analyst, 2015, 140, 20082015 .

40. K. A. Melzak, C. S. Sherwood, R. F. B. Turner and C. A. Haynes, Journal of Colloid and Interface Science, 1996, 181, 635-644.

41. A. Ahniyaz, Y. Sakamoto and L. Bergstrom, Proceedings of the National Academy of Sciences of the United States of America, 2007, 104, 17570-17574.

42. Qiagen DNA Mini Kit, https://www.qiagen.com/us/shop/sample-technologies/dna-sampletechnologies/genomic-dna/qiaamp-dna-blood-mini-kit/). 


\section{Integrated DNA Lysis and Purification of Buccal Swab DNA on a Rotationally-driven Polyethylene-Terephthalate Microdevice}

\section{Summary}

An integrated and disposable polyethylene terephthalate toner $(\mathrm{PeT})$ device is presented that can provide sample-to-PCR ready DNA from dried buccal swab samples in 30 minutes. An on-board lysis protocol and an improved spin-dSPE system were separately validated to yield STR profiles comparable to a conventional method. In validating the extraction step, we found that the spin-dSPE system has a large bandwidth and can obtain STR profiles for samples containing between $30 \mathrm{pg} / \mu \mathrm{L}$ and $20 \mathrm{ng} / \mu \mathrm{L}$ of DNA. The lysis and extraction steps were then integrated on one device and successfully processed up to four samples simultaneously to yield PCR-ready DNA.

\subsection{Introduction}

Biological samples obtained from crime scenes can contain numerous PCR inhibitors that negatively affect DNA analysis, therefore, purification is a key sample preparation step in DNA processing. Phenol-chloroform extractions were popular in the early stages of DNA purification, as restriction fragment length polymorphism (RFLP) required high molecular weight DNA ${ }^{1}$. During this process, DNA is separated from proteinaceous components by exploiting differences in solubility between an aqueous and an organic (phenol/chloroform) phase. As electrophoretic technology advanced, the requirement for high molecular DNA dissipated, opening the door for use of other, less labor-intensive extraction methods that circumvented the use of harsh and toxic chemicals ${ }^{2}$. 
Solid phase extraction (SPE) is one of the most popular DNA extraction methods in forensic labs and was first introduced in 1983 by Colpan et al. ${ }^{3,4}$ following the discovery of a high affinity between DNA and silica in $1979^{5}$. This method uses similar chemistry to that of an organic extraction, but replaced the phenol/chloroform solution with a solid silica phase that inherently increased safety ${ }^{2}$. To purify DNA using this method, the following sequence-specific steps are critical: (i) initial disruption of cells, releasing nucleic material into the solution; (ii) adsoprtion of DNA to silica particles in the presence of a chaotropic salt; (iii) washing the bound DNA to eliminate weakly-bound species and cellular material; and, finally, (iv) desorption of DNA in a low salt buffer, such as Tris-EDTA (TE) buffer. Successful SPE requires complete removal of the sample contaminants but also removal of reagents used in the extraction process (guanidine and isopropyl alcohol) that are potent inhibitors of PCR. Current dSPE methods require multiple sample handling and centrifugation steps or rely on expensive and bulky biorobotic instruments.

The current need for an automated, compact, and low cost DNA extraction platform can be found in microfluidics. Inexpensive and simple device fabrication from commercial-off-theshelf (COTS) materials, combined with decreased sample and reagent volumes significantly reduces the cost per extraction relative to commercial extraction systems. Furthermore, centrifugal approaches to microfluidics has simplified the integration of multiple steps (chemical reaction, mobilization, mixing) within a single device using only a rotation-driven manipulation and onchip valving ${ }^{6,7}$. Passive valving, or fluid flow without external syringe pumps and mechanical valves, is attractive as it eliminates the need for bulky external equipment. Only one report describes a centrifugal microdevice for a nucleic acid extraction that has combined centrifugal microfluidics with complete passive valving 8 . In this report, 'DNA extraction' from whole blood, 
however, involved a physical separation of cellular material and DNA which did not yield DNA that was PCR-ready.

Whole blood in liquid form is the 'universal sample' in clinical diagnostics and, owing to its complex composition, is a frequent standard for demonstrating DNA extraction efficiency. That said, it is not as relevant to forensics where dried samples (blood stains or buccal swabs) are more commonly used ${ }^{1}$. Although centrifugal devices have all of the characteristics needed for chipbased SPE, it has not been demonstrated with buccal swab DNA.

Perhaps more importantly is potential for the development of a micro total analysis system ( $\mu$ TAS), which strives for the full integration and automation of multiple sample processes (e.g., lysis and amplification) on a single microfluidic device. This sample in-answer-out approach has the potential to significantly decrease the footprint of an instrument yet increasing sample throughput and minimize analytical time ${ }^{9,10}$. Several integrated devices have been reported to accommodate buccal swab samples for rapid DNA processing ${ }^{11-13}$, but are limited by bulky external hardware for fluidic control.

Using rotationally-driven flow and solely passive valving, we present the first integrated polyester toner (PeT) microdevice that yields purified DNA from buccal swab samples. In efforts to work towards a $\mu \mathrm{TAS}$, we will validate a sample lysis protocol for on-chip use and demonstrate multiplexed sample processing can provide full short tandem repeat (STR) profiles from a broad concentration range of buccal swab samples. With four extractions per device and full passive valving, the dSPE system is an inexpensive and possibly portable alternative to conventional extraction systems. 


\subsection{Materials and Methods}

\subsubsection{Reagents}

A Qiagen EZ1 DNA Investigator kit (Qiagen, Valencia, CA) was used for all Qiagen EZ1 extractions. For extractions on the microdevice, a 1X Tris-EDTA (TE) buffer (pH 8.0) and an $80 \%(\mathrm{wt} / \mathrm{wt}$ ) isopropanol (Sigma Aldrich, St. Louis, MO) solution were prepared in deionized water. Guanidine Hydrochloride (GuHCl) (MP Chemicals, Santa Ana, CA) was made to concentrations of $3 \mathrm{M}$ and $6 \mathrm{M}$ and adjusted to $\mathrm{pH} 6.1$. A stock solution of Magnesil® paramagnetic particles (Promega, Madison, WI) was diluted 3.3fold in the $3 \mathrm{M} \mathrm{GuHCl}$ solution.

\subsubsection{Device design and fabrication}

Architectural designs were all performed within AutoCAD software (AutoCAD, Autodesk Inc., USA). PeT device fabrication details are described elsewhere ${ }^{14}$. Briefly, a 'print, cut, laminate' method was used for fabrication of all microdevices using a Brother HL-4570CDW (Brother International Corporation, Bridgewater, NJ), a $50 \mathrm{~W} \mathrm{CO}_{2}$ trophy etcher (Universal Laser Systems, Scottsdale, AZ ;VLS3.50), and an office laminator (Mega Electronics; Model 305 laminator; Linton, Cambridge).

\subsubsection{Modified lysis validation}

Samples lysed for initial validation were either a $1 / 8$ buccal swab cutting or a $3 \mathrm{~mm}$ x 3 mm square cutting from a blood soaked Whatman® reference FTA® card (Sigma-Aldrich, Carlsbad, CA) using a previously-described $28 \mu \mathrm{L}$ modified lysis protocol ${ }^{15,16}$. This lysis is a mixture of $18 \mu \mathrm{L}$ of $6 \mathrm{M} \mathrm{GuHCl}(\mathrm{pH} 6.1), 10 \mu \mathrm{L}$ of Qiagen Proteinase $\mathrm{K}$, and the sample of 
interest. The sample is placed in a polyethylene tube and incubated at $56{ }^{\circ} \mathrm{C}$ for 10 minutes. It should be noted that the piggyback method, described elsewhere ${ }^{17,18}$, was used to remove all liquid from the modified method samples following a lysis. Briefly, a thin needle pierced the bottom of the lysis tube before placement in a $1.5 \mathrm{~mL}$ polypropylene tube. Each sample was then centrifuged briefly at $>8,000$ revolutions per minute (RPM) to separate the lysate from the cotton material. A modified $14 \mu \mathrm{L}$ lysis protocol for use on a microdevice used the same modified protocol, but simply reduced the total volume by $50 \%$. It should be noted that all lysis samples were run in parallel with a reagent blank to validate that none of the sample reagents were contaminated. Qiagen manufacturer instructions were used in a conventional lysis. Briefly, a $190 \mu \mathrm{L}$ of Qiagen Buffer G2 (cat \# 1014636), $10 \mu \mathrm{L}$ of Qiagen Proteinase K (cat \# 19131), and the sample of interest were placed in a $2 \mathrm{~mL}$ lysis tube prior to an incubation at $56{ }^{\circ} \mathrm{C}$ for a minimum of 30 minutes.

\subsubsection{On-board lysis protocol}

For preliminary on-chip lysis, 1/8 buccal swab cuttings were placed inside of the lysis chamber prior to running the device through an office laminator to seal the PeT device $\left(130{ }^{\circ} \mathrm{C}\right)$. A small piece of $19 \mathrm{~mm}$ Scotch Magic ${ }^{\mathrm{TM}}$ Tape (3M, St. Paul, MN) was placed over the $2.25 \mathrm{~mm}$ circular opening of the tape valve and secured shut with tweezers. A $15 \mu \mathrm{L}$ aliquot of a premixed lysis cocktail, containing $\mathrm{GuHCl}$ and Proteinase $\mathrm{K}$, was pipetted into the lysis chamber before device placement on a hot plate set to $59^{\circ} \mathrm{C}$ for a 10 minute incubation. Once finished, the device was secured to the edge of a $130 \mathrm{~mm}$ poly(methyl methacrylate) (PMMA) plate on the dSPE system and spun at 1,000 RPM for 60 seconds. A pipette tip was used to puncture through the polydimethylsiloxane (PDMS) film covering the recovery chamber to retrieve the sample.

For on-chip lysis to be integrated with on-chip dSPE, a swab cutting was first cut to the approximate size of the open window of the lysis chamber. The swab cutting was divided into 
quarters and placed in the lysis chamber. Microamp clear adhesive tape (Applied Biosystems, Grand Island, NY) was used to seal the lysis chamber shut. A $15 \mu \mathrm{L}$ aliquot of the premixed lysis cocktail was pipetted into the lysis chamber and then devices were placed on a heat plate set to 59 ${ }^{\circ} \mathrm{C}$. After a 10 minute incubation, the devices were placed on the spin-dSPE system and spun at $1500 \mathrm{RPM}$ for 1 minute $(2.5 \mathrm{~Hz})$ to transfer the aliquoted amount of lysate to a separate chamber. The recovery chamber was punctured with a pipette tip to recover the lysate for further analysis.

\subsubsection{DNA extraction procedures}

All on-chip DNA extractions were run in parallel with a conventional method. These reference methods included the Qiagen DNA mini kit or the EZ1 DNA investigator kit on an automated Qiagen EZ1 Advanced instrument (Qiagen, Valencia, CA). A full reference buccal swab was extracted with the DNA mini kit for reference STR profiles according to manufacturer instructions. To purify samples with the EZ1 instrument, a $2 \mu \mathrm{L}$ aliquot from each lysed sample was diluted to a volume of $200 \mu \mathrm{L}$ with $6 \mathrm{M} \mathrm{GuHCl}$ (pH 6.1; MP Chemicals, Santa Ana, CA) in a $2 \mathrm{~mL}$ microtube. The samples were placed in the EZ1 instrument according to manufacturer instructions using a 'trace - tip dance protocol' with a $50 \mu \mathrm{L}$ Tris- EDTA (TE) elution volume. Once samples were finished, they were stored at $4{ }^{\circ} \mathrm{C}$ until needed for further processing.

To run the samples on the spin-dSPE system, $2 \mu \mathrm{L}$ of a lysed sample is added to the DNA binding chamber of a pre-reagent loaded device. Once the sample volume is added, a saved propeller automated program begins the extraction process. First, a mixing process, described elsewhere ${ }^{19}$, is initiated. Briefly, an array of stationary permanent magnets is brought to $9 \mathrm{~mm}$ from a sample disc followed by bi-directional swaying of the sample disc for 500 seconds. Once the mixing was completed, the magnetic disc was brought to 
maximum height $(49 \mathrm{~mm})$ from the sample disc to start the fluidic control process. The following optimized spin parameters were used during on-chip extraction: (i) IPA wash: 310 RPM 110 seconds, $0.67 \mathrm{~Hz}^{*} \mathrm{~s}^{-1}$, clockwise; (ii) TE wash: $450 \mathrm{RPM}, 10$ seconds, 0.67 $\mathrm{Hz}^{*} \mathrm{~s}^{-1}$, clockwise; and (iii) loading TE elution buffer: $500 \mathrm{RPM}, 1$ second, $0.33 \mathrm{~Hz}^{*} \mathrm{~s}^{-1}$, clockwise. A second mixing step, to desorb the DNA from the silica beads, was initiated and is the same method as described previously. Finally the eluted DNA was transferred to a recovery chamber by spinning the motor at $1000 \mathrm{RPM}, 1$ second, $1.5 \mathrm{~Hz}^{*} \mathrm{~s}^{-1}$, counterclockwise. A clean SGE liquid syringe (cat \# 24845, Restek, Bellefonte, PA) was used to puncture the polymer covering the DNA recovery chamber and pull the purified DNA from the microdevice.

\subsubsection{Post processing procedure}

Following separate lysis and extraction validation experiments, the DNA concentration was obtained for each sample both on a 7500 Real-Time PCR system (Applied Biosystems®, Foster City, CA) using a Plexor® HY kit (Promega, Madison, WI) and a Qubit ${ }^{\circledR} 2.0$ fluorimeter, with a high sensitivity kit, according to manufacturer instructions. Once the concentration of each sample was known, all samples were amplified using AmpFISTR® Identifiler® Plus kits (Middletown, VA) targeting 1 ng total DNA per sample. An ABI 3130xl genetic analyzer (Applied Biosystems ${ }^{\circledR}$, Foster City, CA) was used for obtaining all electropherograms post amplification. For integrated lysis and extraction experiments, samples were amplified using a Promega PP18D STR kit (Fitchburg, WI) targeting 1 ng total DNA per sample and analyzed on an ABI 310 genetic analyzer (Applied Biosystems ${ }^{\circledR}$, Foster City, CA). 


\subsection{Results}

\subsubsection{Microdevice extraction design improvements}

As defined by Jackson et al. ${ }^{20}$, the dSPE microdevice demonstrated the proof-ofprinciple DNA purification from whole blood samples, but required manual valving operations that limited its use in application in a real world environment. Several improvements were made to increase the automation of the system which includes the exchange of a manually actuated valve for a passive resistive valve and the addition of a second Tris-EDTA (TE) and a third waste chamber (Fig. 1). Similar to a siphon valve, the resistive valve is rotationally controlled and requires solutions to overcome the siphon crest before it can proceed to the waste chambers. However, the valve relies on pneumatic pressure produced from centrifugal force rather than capillary action. This allows for increased rotational control at low rotational speeds ( $<500$ RPM) but increases the risk for

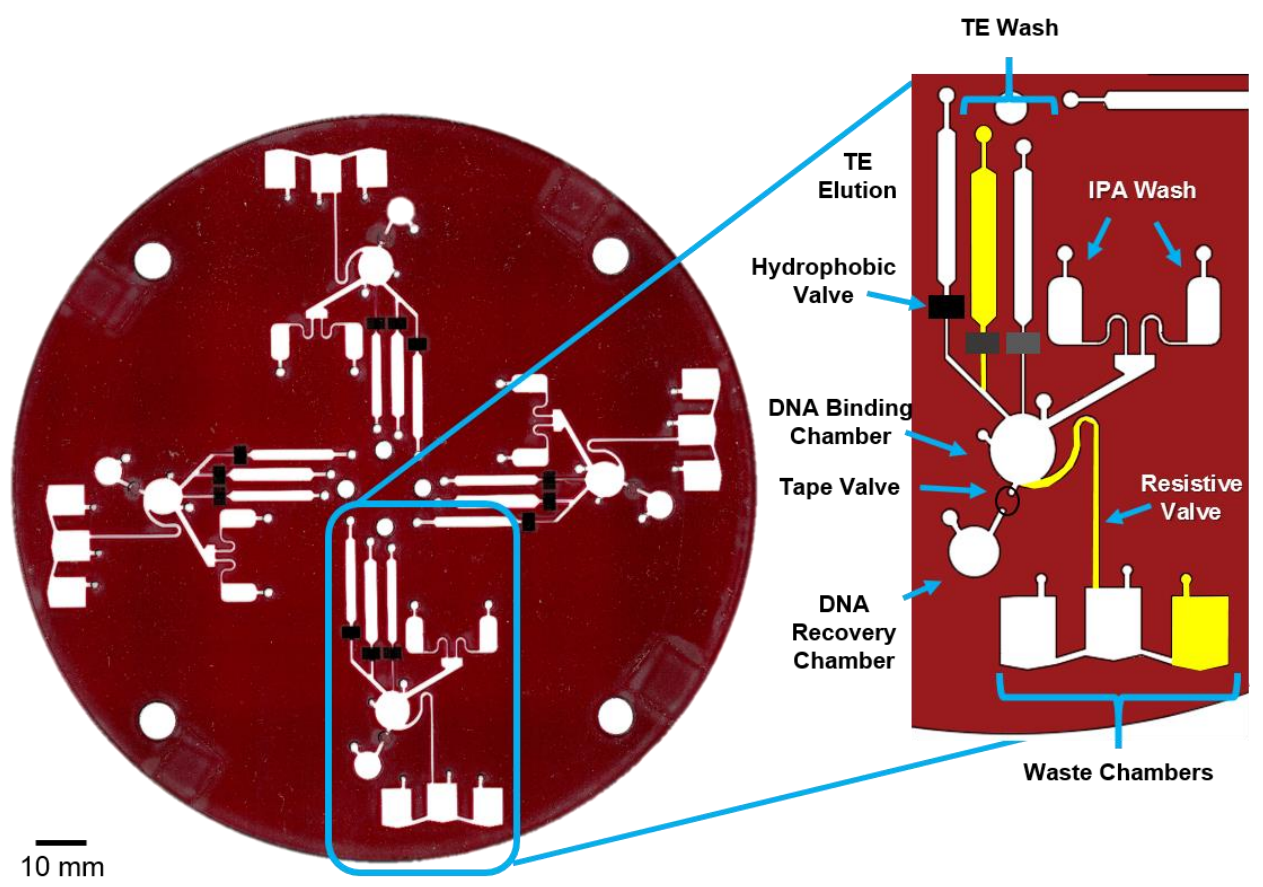

Figure 1: Full view of the dSPE improved design with labelled sections of one dSPE domain. Highlighted seconds are improvements to the dSPE device design. 


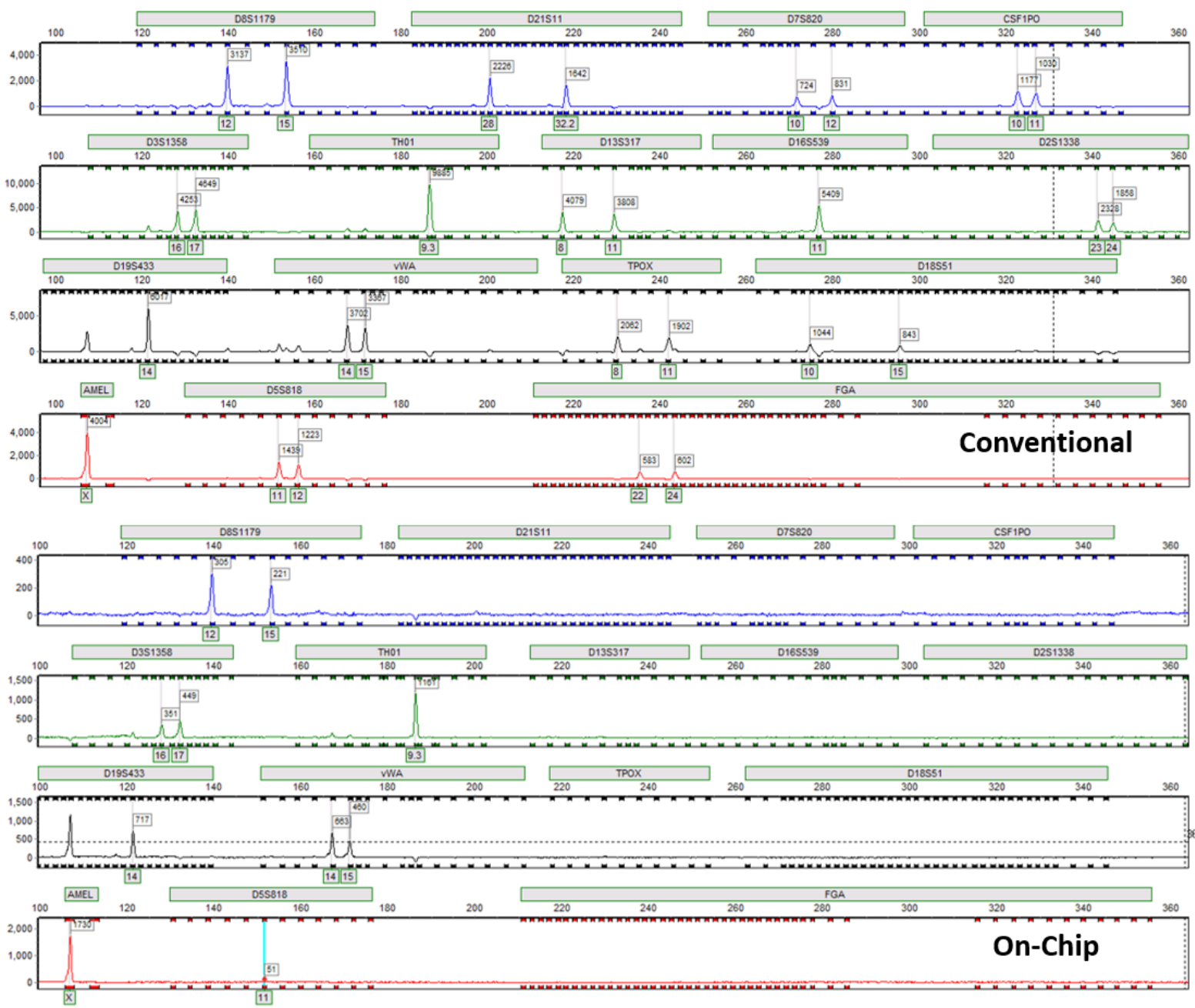

Figure 2: STR profile comparison of a conventional Qiagen extraction to a partial STR profile following an on-chip extraction with PCR inhibition due to residual IPA.

undesired residual inhibition (from $\mathrm{GuHCl}$ and/or IPA) in the DNA binding chamber following a wash. Although this residual fluid can help to re-wet the resistive valve for easier wicking of a secondary fluid, these inhibitors compromise the PCR-readiness of the DNA (Fig 2). To circumvent this issue, a second TE wash chamber (total volume of 7.5 $\mu \mathrm{L}$ ) was added to the original dSPE design to help flush all residual contaminants (IPA and $\mathrm{GuHCl}$ ) from the DNA binding chamber through to the waste chambers. Finally, a third waste chamber was added due to increased wash volumes. Despite the architectural 
changes, four identical extraction domains remain on each device, with the capability of accepting up to 4 separate buccal wash samples per device.

\subsubsection{Optimization of On-chip dSPE Protocol}

Previously optimized parameters were re-evaluated for each of the following dSPE steps following architectural improvement: (i) binding DNA to magnetic silica particles; (ii) washing with IPA to eliminate $\mathrm{GuHCl}$ and poorly bound proteinaceous material; (iii) washing with TE buffer to minimize IPA; (iv) introduction of TE elution buffer to the binding chamber; (v) desorption of DNA from the silica particles; and (vi) transport of purified DNA to the DNA recovery chamber. As the location and dimensions of the binding chamber remained the same, there was no need to change the previously-optimized mixing protocol to bind and elute DNA from the silica particles ${ }^{20}$.

The insertion of a second TE buffer chamber required the re-optimization of all hydrophobic valves, or patches of toner lining the top and bottom of all TE chambers. These passive valves enable sequential release of each of the TE wash steps followed by the TE elution buffer which is critical during a given DNA extraction. The burst pressure of these hydrophobic

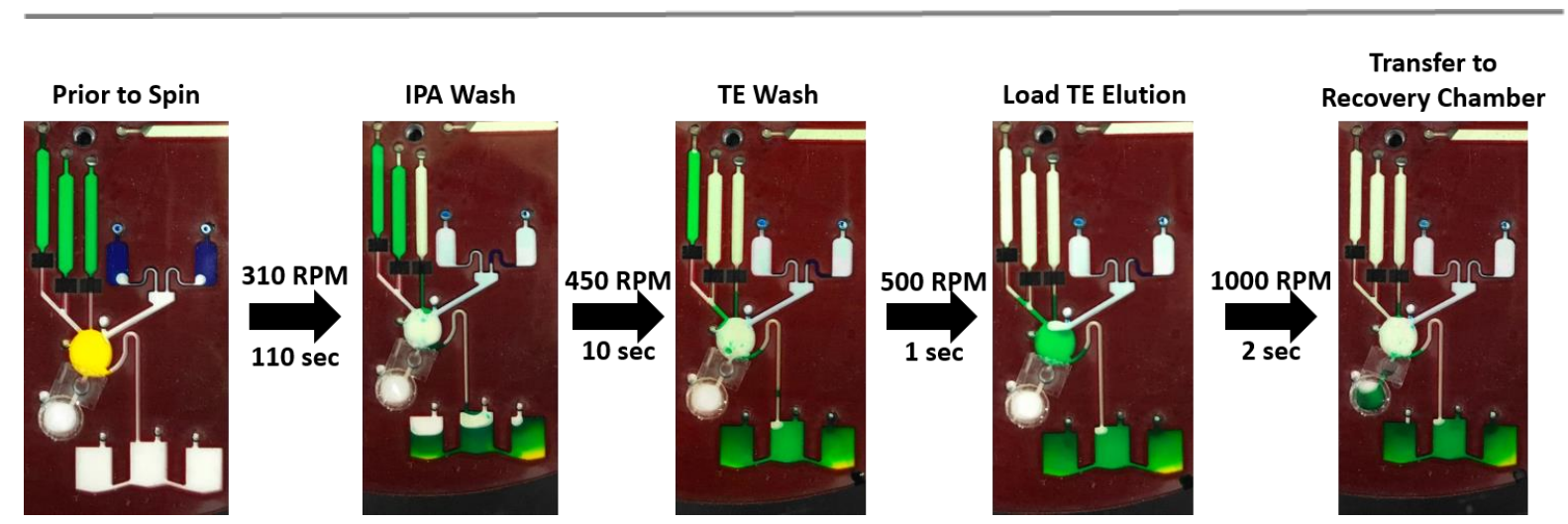

Figure 3: Optimized spin protocol for the improved dSPE design with accommodating device snapshots. 
valves were tuned by changing the grayscale (or hydrophobicity) of the toner patch and the distance from the center of rotation ${ }^{21}$. The burst frequency of the first TE wash was set to release just after the release of the IPA wash (310 RPM). This first TE wash helped push the residual IPA wash solution to the waste chambers. As residual IPA may still be present in the DNA binding chamber after the first TE wash, a second TE wash was initiated at 450 RPM. Following the TE wash, the only fluid remaining in the DNA binding chamber is residual TE buffer, which does not inhibit PCR (Fig. 3).

To elute the DNA from the beads, it was necessary to trap the TE elution buffer in the binding chamber following all IPA and TE wash steps. Since the elution buffer is the same composition as the TE wash solutions, spin frequency and time alone would not prevent the elution buffer (once released from the hydrophobic valve) from passing through the resistive valve. Instead, the waste chamber was used to create fluidic resistance to keep the TE elution buffer in the binding chamber. To do this, the waste chamber was designed to match the total volume of all binding and wash buffer volumes. When the waste chamber was full, it would mimic a closed channel due to the blockage of air flow, thus preventing the TE buffer from moving beyond the binding chamber. By decreasing the acceleration of the spin step that released the TE elution buffer, it improved the rate of successful fluid trapping. With the updated dSPE design, the success rate of trapping the elution volume within the DNA binding chamber, was $77 \%(\mathrm{n}=40)$, as determined by a high-speed camera. Deviations to this pattern were attributed to small variations in channel architecture and reagent evaporation. To minimize evaporation, we explored the use of $80 \%$ 1-butanol and $80 \%$ 2-butanol (v/v) instead of IPA. In triplicate testing of two donors, we found that 1- and 2-butanol is as efficient with extracting DNA from a sample and does not 
negatively affect an STR profile (Fig. 4). In substituting 1-butanol for IPA, the reproducibility increased to $90 \%(n=5)$.

\subsubsection{Comparing DNA Extraction Methods}

The performance of microdevice sample extractions was accessed by comparing the STR profiles from on-chip purified samples to those purified from a conventional method. For this comparison, we chose to use the Qiagen EZ1 Advanced biorobotic instrument because it is one of the fully automated systems currently validated for use with forensic casework samples. Since the spin-dSPE system can extract up to 4 samples simultaneously, one microdevice device was used
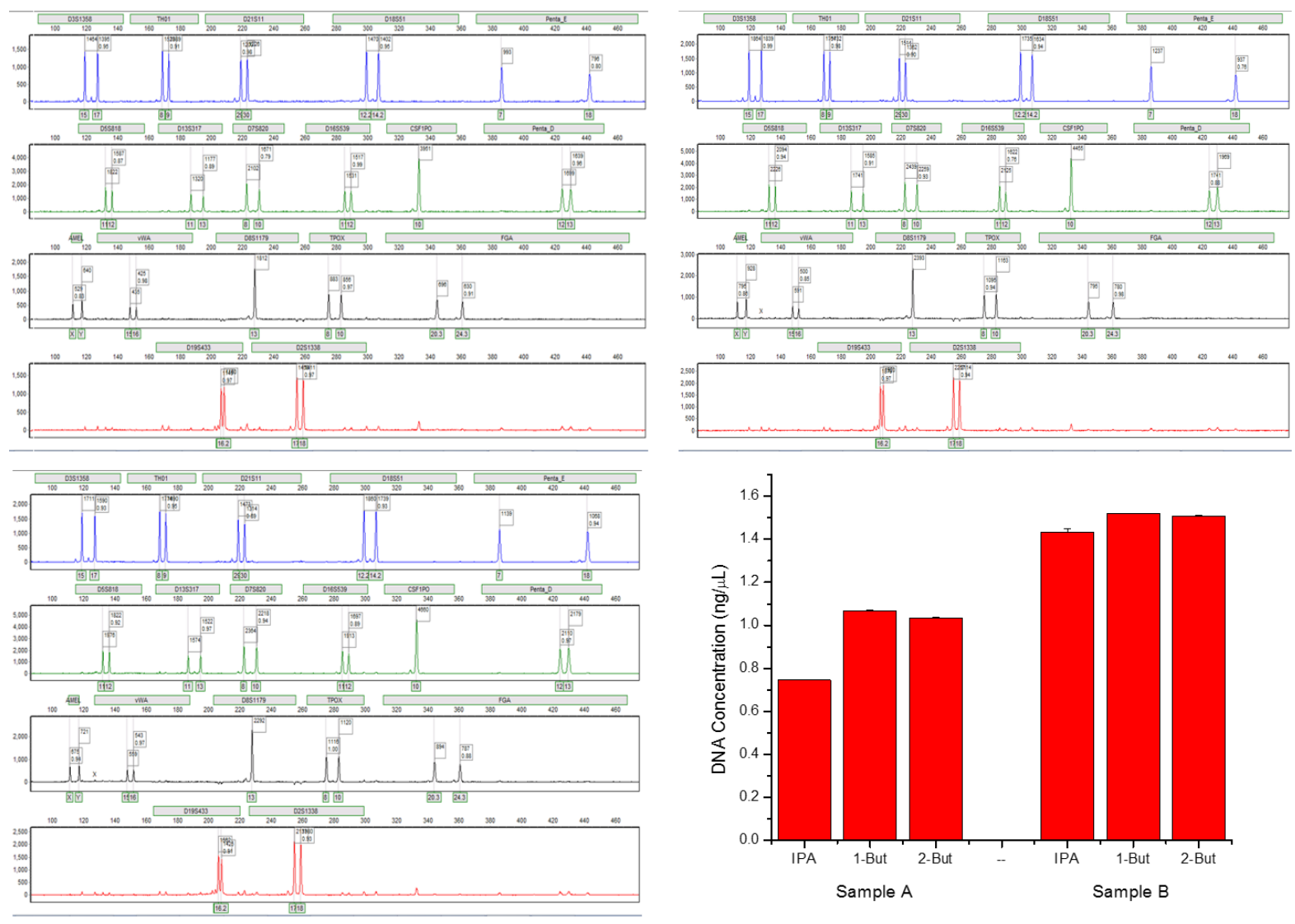

Figure 4: STR comparisons following the exchange of isopropyl alcohol (IPA) for 1butanol (1-But) and 2-butanol (2-But). 
to obtain replicate extractions from one sample in 20 minutes. As shown in Figure $\mathbf{5}$, preliminary experiment results show that a comparable amount of DNA can be extracted from the spin-dSPE microdevice. When these samples were amplified, all samples provided strong STR profiles, with microdevice extractions having similar peak heights and a $100 \%$ correlation to the reference EZ1 extracted and amplified samples.

To better access the applicable bandwidth of the spin-dSPE system, multiple buccal swab samples of unknown concentrations were tested. Buccal swabs from three different donors were sampled in addition to $1: 2$ and 1:4 sample dilutions. As
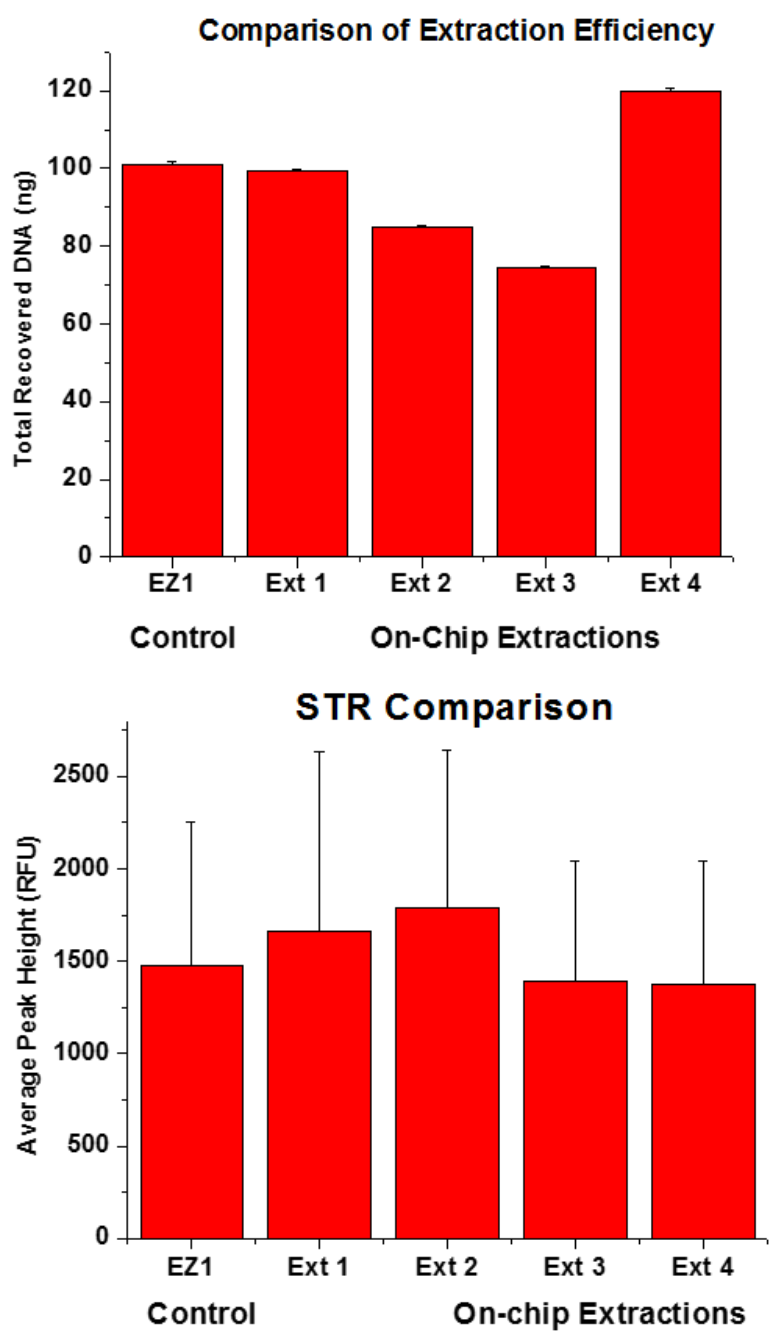

Figure 5: Replicate on-chip extraction efficiency and STR profile comparisons to a conventionally used Qiagen EZ1 instrumental method.

shown in Figure 6 strong STR profiles were obtained for on-chip extractions and correlated to those obtained from EZ1 extractions. Donor 1 tested the lower limitations of the spin-dSPE system, with a 1:4 dilution containing a total of $300 \mathrm{pg}$ of DNA post extraction. Despite this, the STR profile from the spin-dSPE system was comparable to that using an EZ1 and with 100\% correlation. 


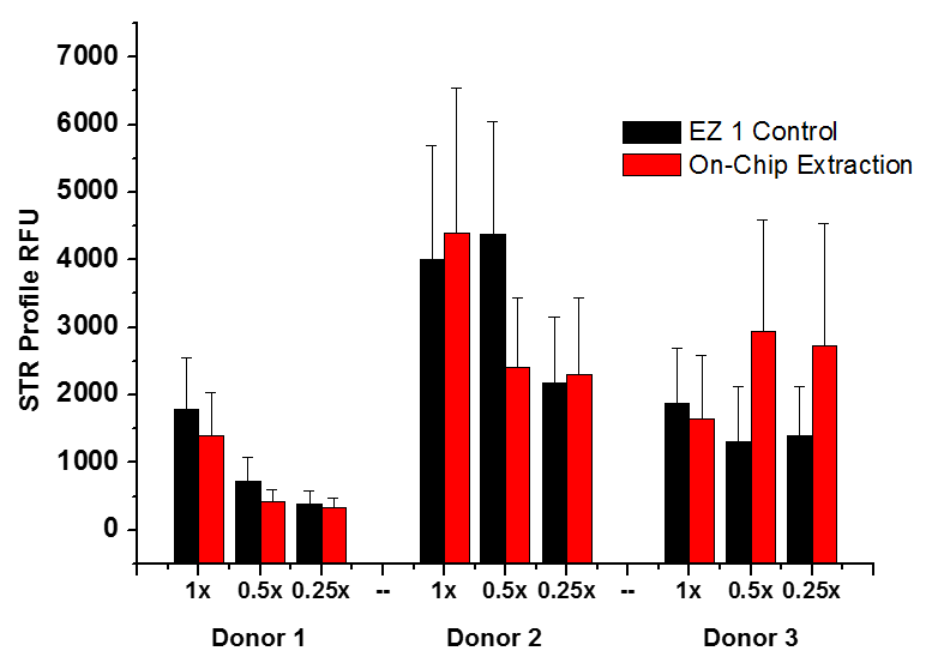

Figure 6: STR profile comparison of on-chip extractions to a conventional Qiagen EZ1 extraction method for multiple donors at various concentrations.

\subsubsection{Potential for PeT on-chip}

lysis

As microfluidic systems

strive towards integrating and automating multiple processes for a true "lab on a chip", integration of sample lysis to the current spindSPE microdevice was explored.

On-board lysis would benefit the

system by providing a closed sample-to-PCR ready DNA system and minimize the risk for contamination. A previously reported $28 \mu \mathrm{L}$ lysis protocol ${ }^{16}$ was first compared to a conventional Qiagen EZ1 lysis method ${ }^{22-24}$ to determine whether a 10-fold decrease in lysis volume would

Table 1: Validation of New Lysis Method for Forensic Samples

\begin{tabular}{|c|c|c|c|c|c|c|}
\hline \multirow[b]{2}{*}{$\begin{array}{c}\text { Volume } \\
\text { Loci }\end{array}$} & \multicolumn{2}{|c|}{ FTA Card } & \multicolumn{2}{|c|}{ Buccal Swab } & \multicolumn{2}{|c|}{ Buccal Swab } \\
\hline & $\begin{array}{c}28 \mu \mathrm{L} \\
10 \text { Minute Lysis }\end{array}$ & $\begin{array}{c}200 \mu \mathrm{L} \\
30 \text { Minute Lysis }\end{array}$ & $\begin{array}{c}28 \mu \mathrm{L} \\
10 \text { Minute Lysis }\end{array}$ & $\begin{array}{c}200 \mu \mathrm{L} \\
30 \text { Minute Lysis }\end{array}$ & $\begin{array}{c}14 \mu \mathrm{L} \\
10 \text { Minute Lysis }\end{array}$ & $\begin{array}{c}200 \mu \mathrm{L} \\
30 \text { Minute Lysis }\end{array}$ \\
\hline D8S1179 & 8,10 & 8,10 & 13,15 & 13,15 & 12,13 & 12,13 \\
\hline D21S11 & $30,32.2$ & $30,32.2$ & 30,32 & 30,32 & 29 & 29 \\
\hline D7S820 & 12 & 12 & 9,11 & 9,11 & 10 & 10 \\
\hline CSF1PO & 11 & 11 & 11 & 11 & 7,10 & 7,10 \\
\hline D3S1358 & 15,18 & 15,18 & 18 & 18 & 15 & 15 \\
\hline TH01 & 7,9 & 7,9 & 6,8 & 6,8 & 8,9 & 8,9 \\
\hline D13S317 & 12,13 & 12,13 & 11,12 & 11,12 & 11,12 & 11,12 \\
\hline D16S539 & 11 & 11 & 12,13 & 12,13 & 11,12 & 11,12 \\
\hline D2S1338 & 17,19 & 17,19 & 17,20 & 17,20 & 18,22 & 18,22 \\
\hline D19S433 & 13,14 & 13,14 & 13,16 & 13,16 & 14 & 14 \\
\hline vWA & 16,17 & 16,17 & 18,19 & 18,19 & 15,16 & 15,16 \\
\hline TPOX & 11 & 11 & 11,12 & 11,12 & 8 & 8 \\
\hline D18S51 & 14,15 & 14,15 & 15,18 & 15,18 & $13.2,15$ & $13.2,15$ \\
\hline Amelogenin & $X, Y$ & $X, Y$ & $x$ & $x$ & $X, Y$ & $X, Y$ \\
\hline D5S818 & 12 & 12 & 11,14 & 11,14 & 12,13 & 12,13 \\
\hline FGA & 23,25 & 23,25 & 19,26 & 19,26 & 21,24 & 21,24 \\
\hline $\begin{array}{c}\text { Quant }(\mathrm{ng} / \mu \mathrm{L}) \\
\text { Average Peak Height }\end{array}$ & 0.28 & 0.32 & 0.17 & 0.22 & 0.35 & 0.28 \\
\hline $\begin{array}{c}\text { (RFU) } \\
\text { Standard Deviation } \\
\text { (RFU) }\end{array}$ & $\begin{array}{r}2132 \\
861 \\
\end{array}$ & 1719 & 2987 & 2605 & 2893 & 4013 \\
\hline
\end{tabular}


compromise DNA recovery. Multiple sample types including a dried buccal swab cutting ( 1/8 of a swab) and a $3 \mathrm{~mm}$ square cutting of dried blood on an FTA card were used for the comparison of these two lysing methods. To do this, triplicate modified lysed samples were processed in parallel to a reference EZ1 control to compare the obtained STR profiles. As shown in Table 1, an equivalent amount of DNA was recovered from the $28 \mu \mathrm{L}$ lysis protocol compared to that of the larger $200 \mu \mathrm{L}$ volume, resulting in nearly identical STR profiles.

The lysis volume was further decreased to $14 \mu \mathrm{L}$ with buccal swab samples to observe whether comparable amounts of DNA could still be obtained from a volume amendable to placing on a microdevice. As shown in Table 1, the volume reduction did not impact the amount of DNA recovered, and thus produced a strong STR profile following amplification. For these reasons, all

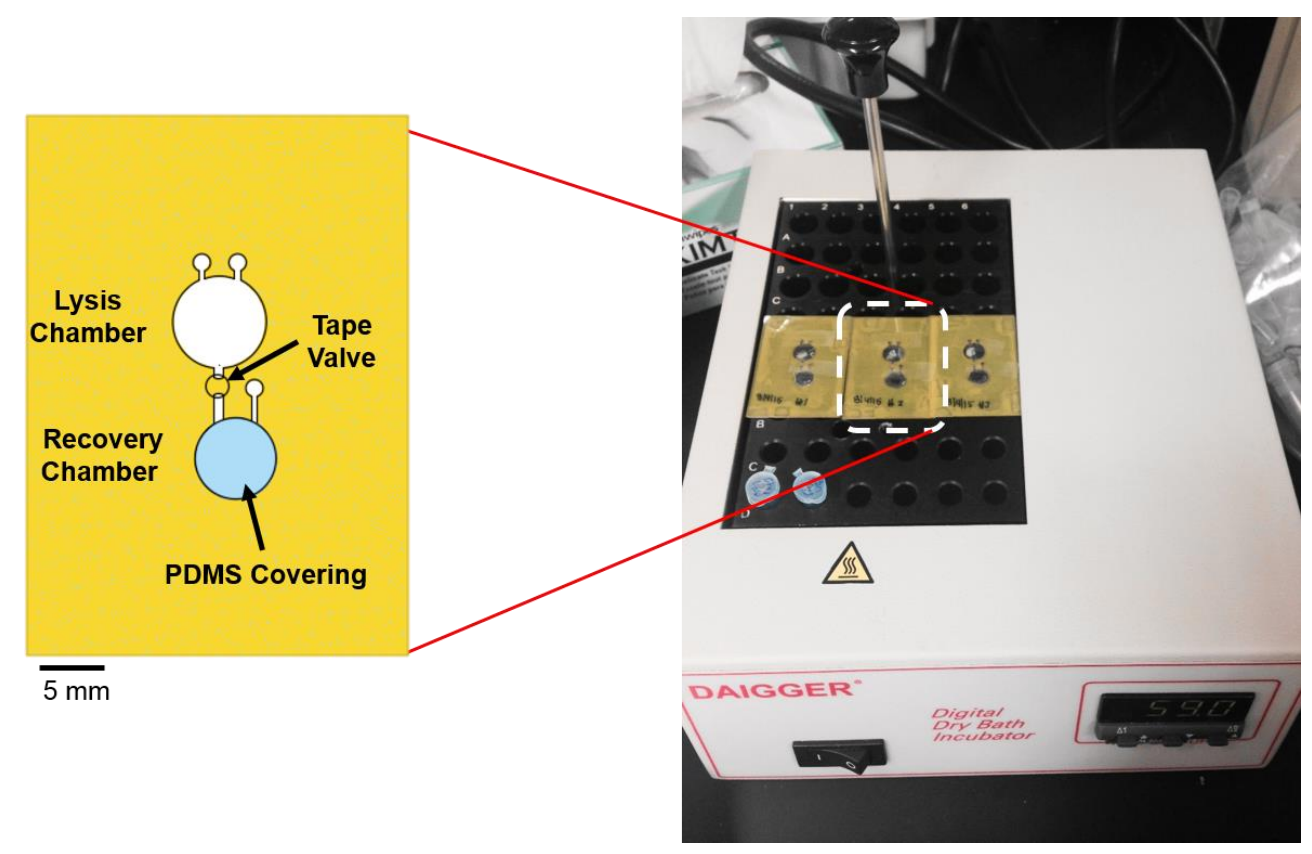

Figure 7: Schematic of On-chip Lysis device with Comparison of DNA samples $(\sim 2$ $\mathrm{ng} / \mu \mathrm{L})$ from Qiagen EZ1 extraction and an on-chip extraction with a reagent blank control. 
future sample lysis was done using a $14 \mu \mathrm{L}$ lysis sample volume and a 10 minute incubation at $56^{\circ} \mathrm{C}$.

A simple PeT device with a lysis and recovery chamber was used for initial on-board lysis experiments to determine the degree of DNA recovery compared to conventional tube extractions. For this experiment, replicate microdevices were filled with a premixed lysis cocktail and placed on a heat block alongside multiple tube controls for a 10 minute incubation at $56{ }^{\circ} \mathrm{C}$ (Fig. 7). Following sample lysis, each microdevice was placed on the spin-dSPE platform to transfer the lysis buffer to a recovery chamber. The lysate was retrieved by puncturing the polydimethylsiloxane (PDMS) layer covering the recovery chamber. Each lysate was then diluted to $200 \mu \mathrm{L}$ with $6 \mathrm{M} \mathrm{GuHCl}$ and extracted on an EZ1 instrument. DNA quantitation of these samples indicated that the modified $14 \mu \mathrm{L}$ tube lysis controls yielded $0.1857 \mathrm{ng} / \mu \mathrm{L}$ and 0.5912 $\mathrm{ng} / \mu \mathrm{L}$ DNA while the on-chip sample lysis yielded $0.1131 \mathrm{ng} / \mu \mathrm{L}, 0.2119 \mathrm{ng} / \mu \mathrm{L}$ and $0.1895 \mathrm{ng} / \mu \mathrm{L}$ DNA, respectively. As each of these samples contain different buccal swab cuttings, the

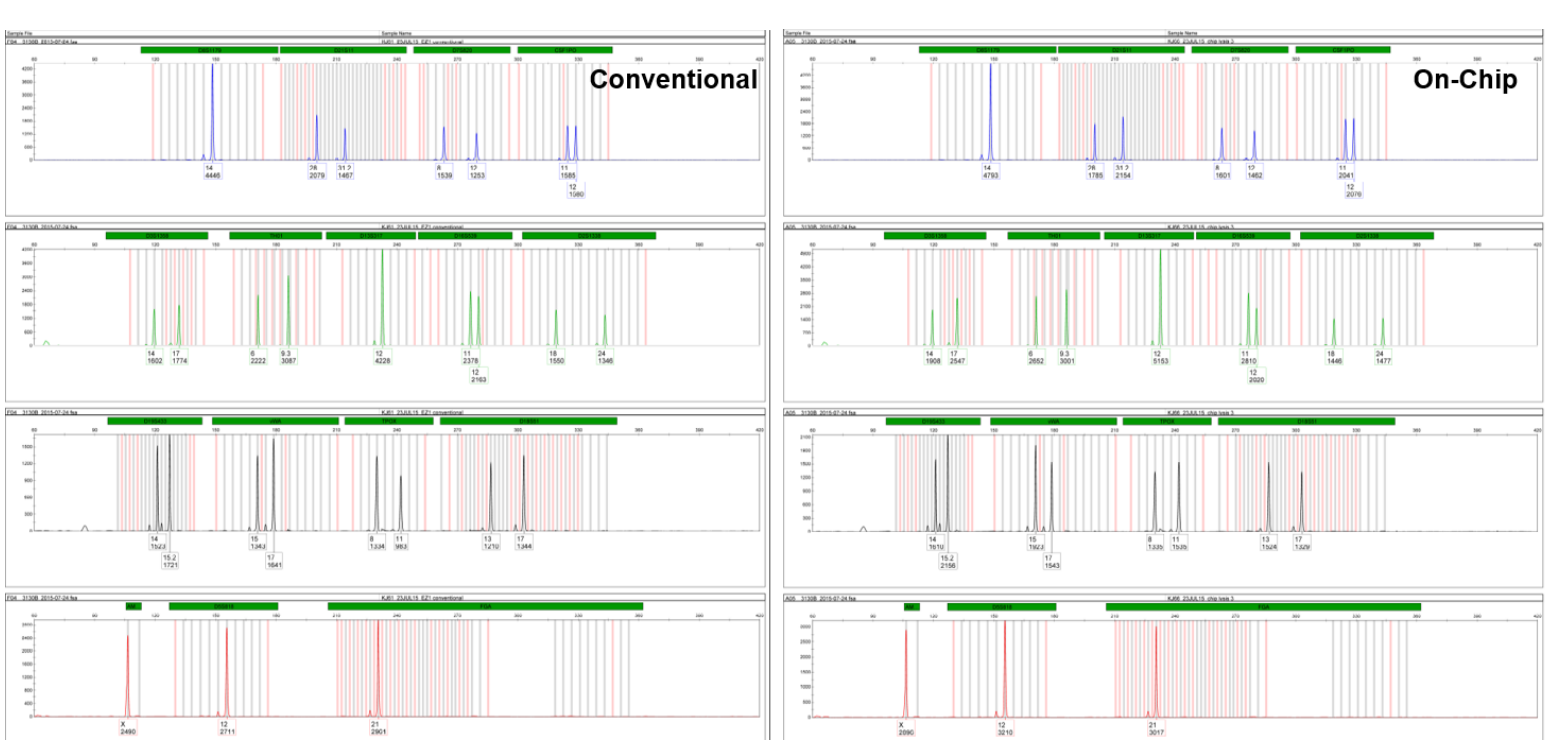

Figure 8: STR profile comparison between an on-chip lysis of a buccal swab and a conventional tube lysis. 
concentration of the extracted DNA cannot be expected to be of the same value. However, a slight decrease in DNA recovery on a microdevice could be attributed to a $25 \%$ loss in lysis volume due to swab absorption. Despite this, full STR profiles could be obtained for each sample, with 100 $\%$ correlation to those obtained from the tube controls. An exemplary example of this comparison is shown in Figure 8.

With data supporting that on-board lysis is feasible, a sample lysis device was designed that is easily integrated to the current dSPE device design (Fig. 9A). There are several unique features which were exploited to allow for simple and effective DNA lysis. A rectangular cutout in the face of the lysis chamber was advantageous in providing a reference size of the swab cutting for effective lysis (5 mm x $3 \mathrm{~mm})$. A swab cutting larger than the cutout was found to decrease the DNA recovery due to increased fluid absorption which is prevented by using the cutout as a size reference. If the swab cutting is split into quarters before placing into the lysis chamber, there is a $30 \%$ increase in DNA recovery which may be due to the increased swab surface area exposure to the lysis buffer (Fig 10). Other advantages of the cutout include facile sealing with a

A

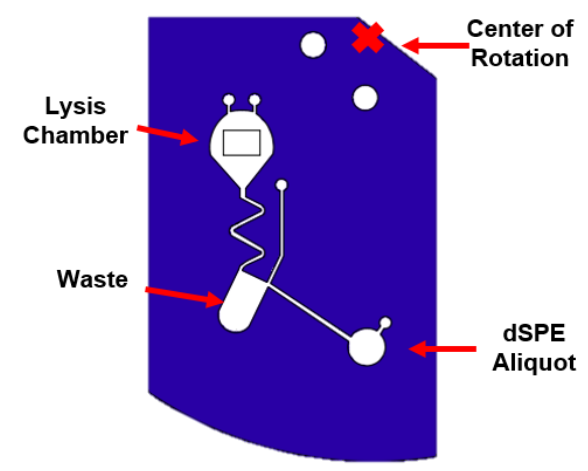

B

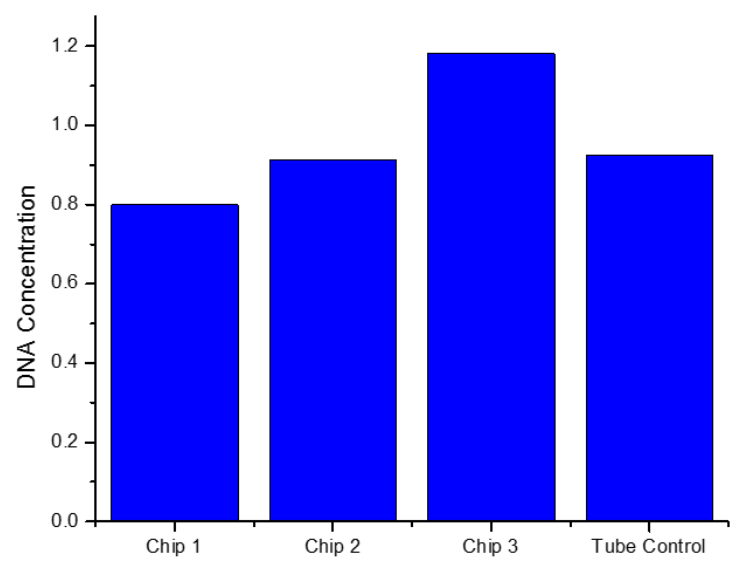

Figure 9: On-chip PeT Lysis Demonstration for Buccal Swab Cuttings. (A)Illustration of microdevice architecture for a sample lysis. (B) Comparison of the DNA recovery from replicate on-chip sample lysis versus a $28 \mu \mathrm{L}$ tube control. 

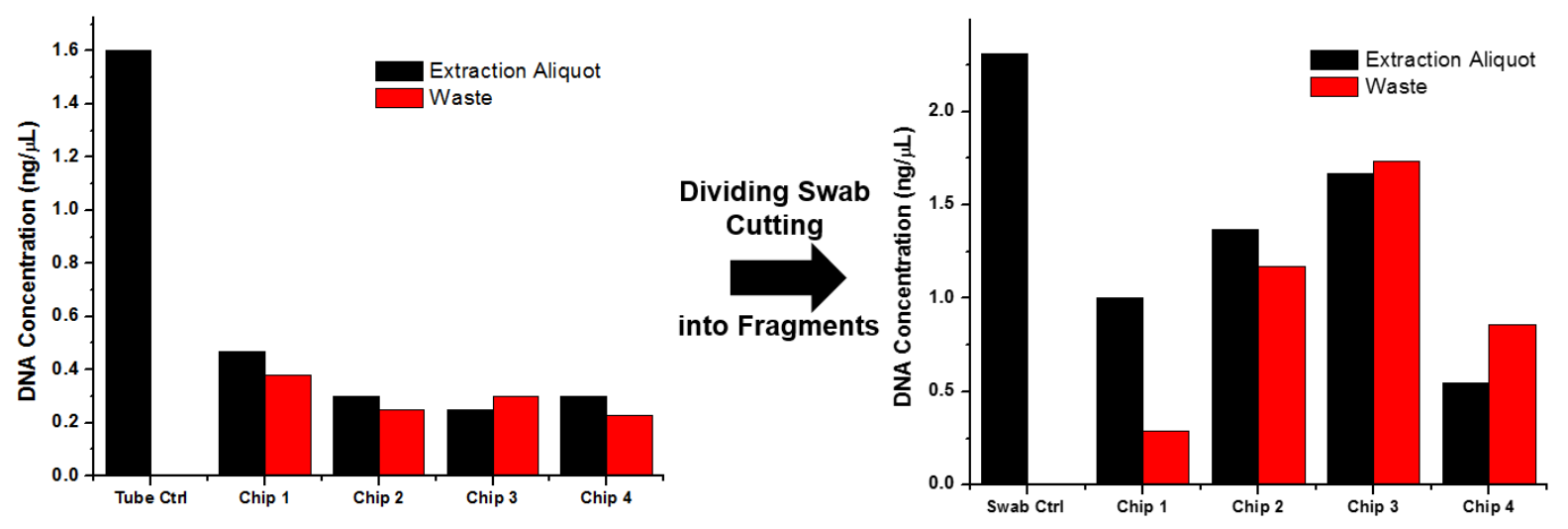

Figure 10: DNA recovery from buccal swab cuttings before and after dividing swab cutting into fragments prior to lysis.

biocompatible adhesive tape for a closed system during on-board lysis while providing easy access to the swab cuttings if they are needed to be recovered from the device post lysis. A serpentine channel, just downstream of the lysis chamber, provides increased mixing of the lysate before entering the dSPE domain. Since dSPE only requires a small aliquot from the lysate, a waste chamber could effectively reduce the lysate volume to be $2-4 \mu \mathrm{L}$ of sample. As shown in Figure 9B, DNA of the similar concentration was recovered from multiple 'dSPE aliquot' chambers post on-board lysis and was similar to an aliquot from a $28 \mu \mathrm{L}$ tube control. Following a Qiagen extraction of all samples, full STR profiles could be obtained from the sample lysis device and was concordant to a reference control sample (Fig. 11).

\subsubsection{Integrated lysis and purification}

The lysis domain was designed according to the free space on the dSPE microdevice so these processes could be easily amalgamated without compromising the number of total sample domains on each device. The dSPE design advancement is shown in Figure 12 from the initial 

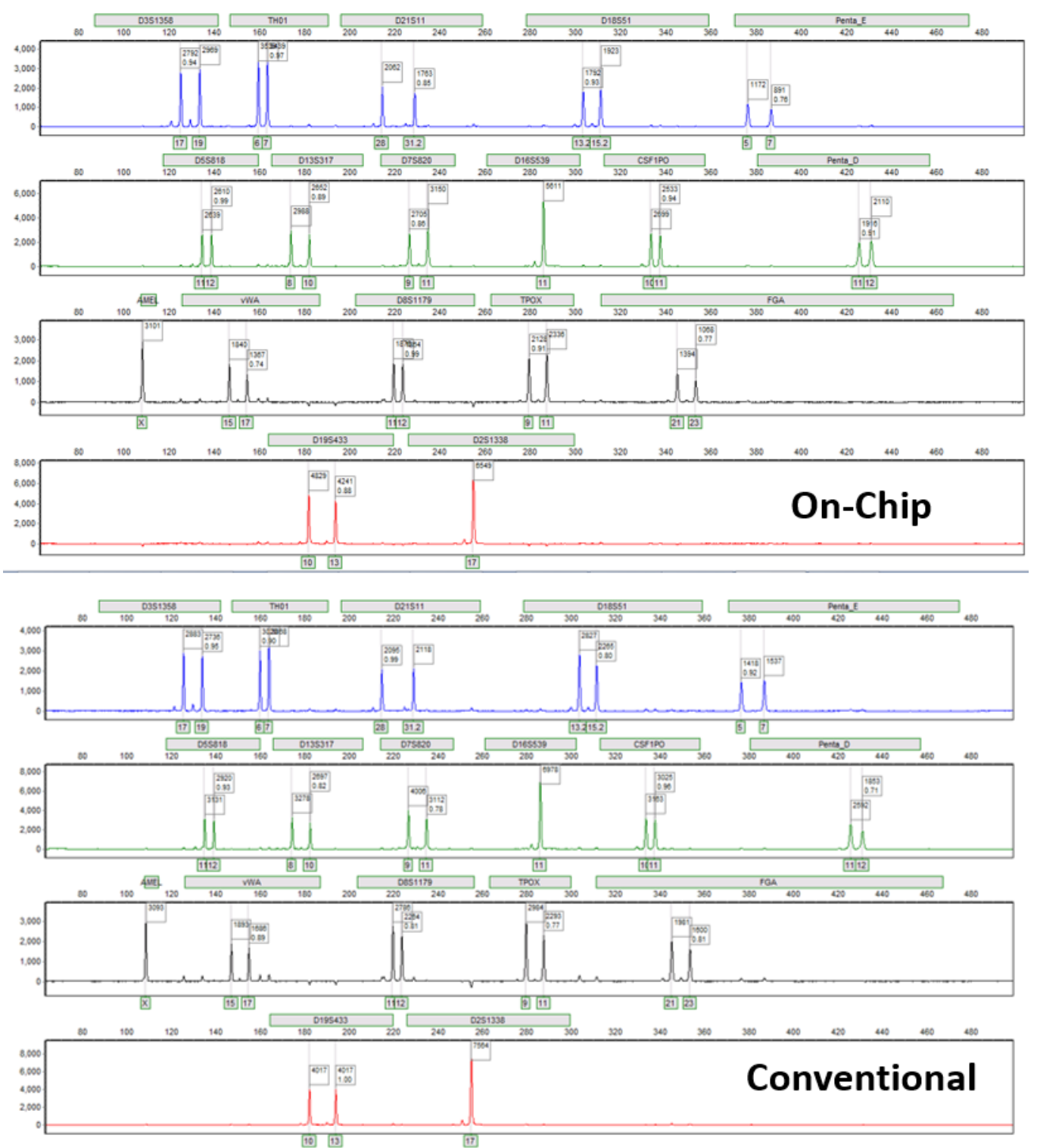

Figure 11: Representative STR profile comparison between a $14 \mu \mathrm{L}$ on-chip sample lysis and a conventional $28 \mu \mathrm{L}$ tube control lysis.

device design, published elsewhere ${ }^{20}$, to the current integrated lysis and extraction microdevice design. The newest design has many advantages including hands-free operation, which eliminates previous manual valve manipulation and lowers the risk for contamination. Initial buccal samples processed with the integrated device yielded strong STR profiles, with an exemplary comparison to a conventional method shown in Figure 13A. Although conventional Qiagen manual extraction 

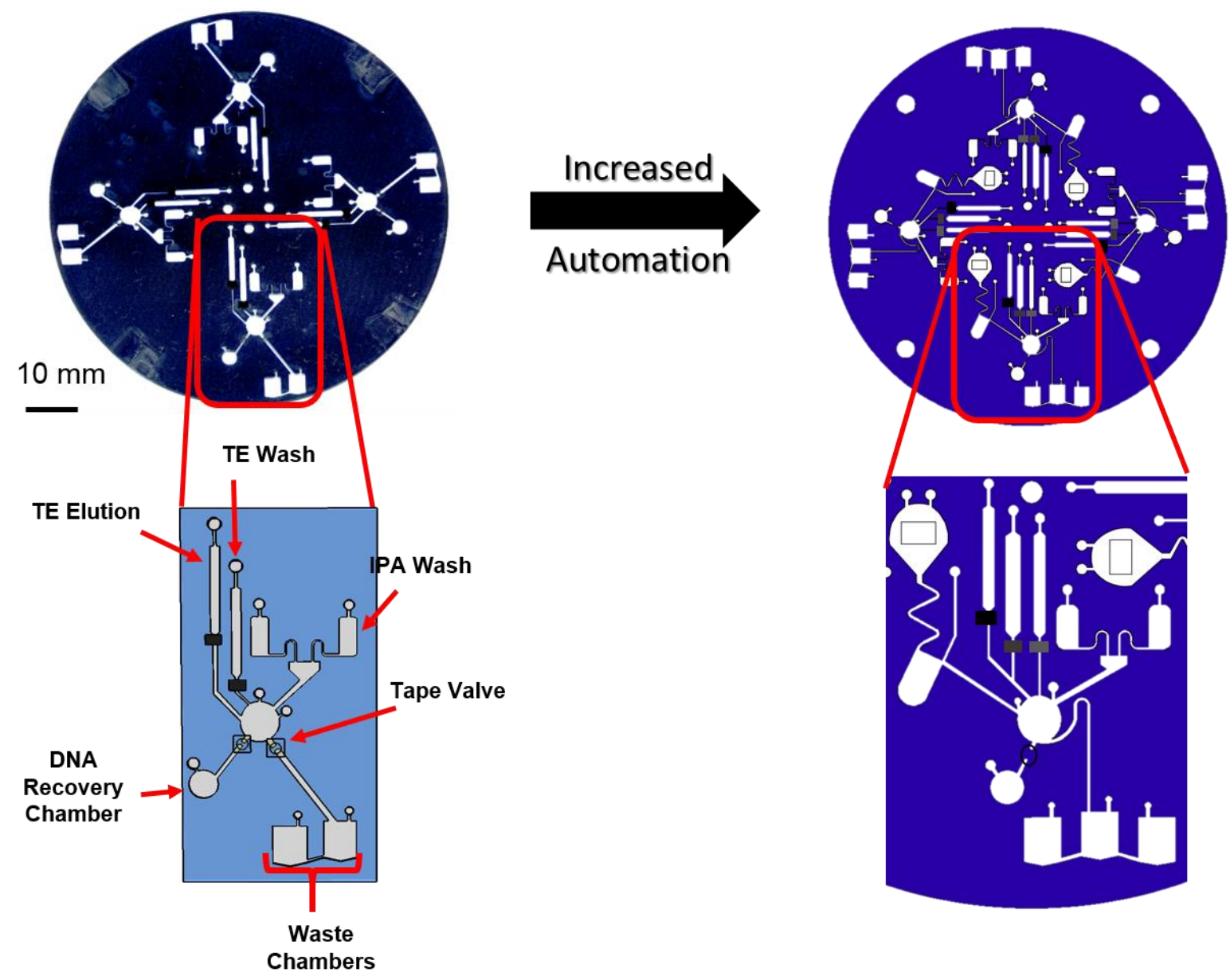

Figure 12: Front-view illustrations of the first edition dSPE device design and an improved design to minimize manual manipulation and integration of the sample lysis.

kits are well validated for DNA purification from buccal samples, the DNeasy kit could not recover enough DNA from 2-4 $\mu \mathrm{L}$ of sample lysate for a full STR profile. Instead, all $28 \mu \mathrm{L}$ of conventional sample lysates were extracted and processed through to obtain STR profiles.

The integrated spin-dSPE system was then challenged with running the lysis and extraction of four separate cutting samples simultaneously. Since the lysis domains are close to the center of the disc, the disc could be placed on one heater plate to lyse all samples. Following the lysis, the disc was placed on the spin system to transfer lysates to the extraction chambers and purify according to the dSPE protocol previously discussed. As shown in Figure 13B, each of these 
A
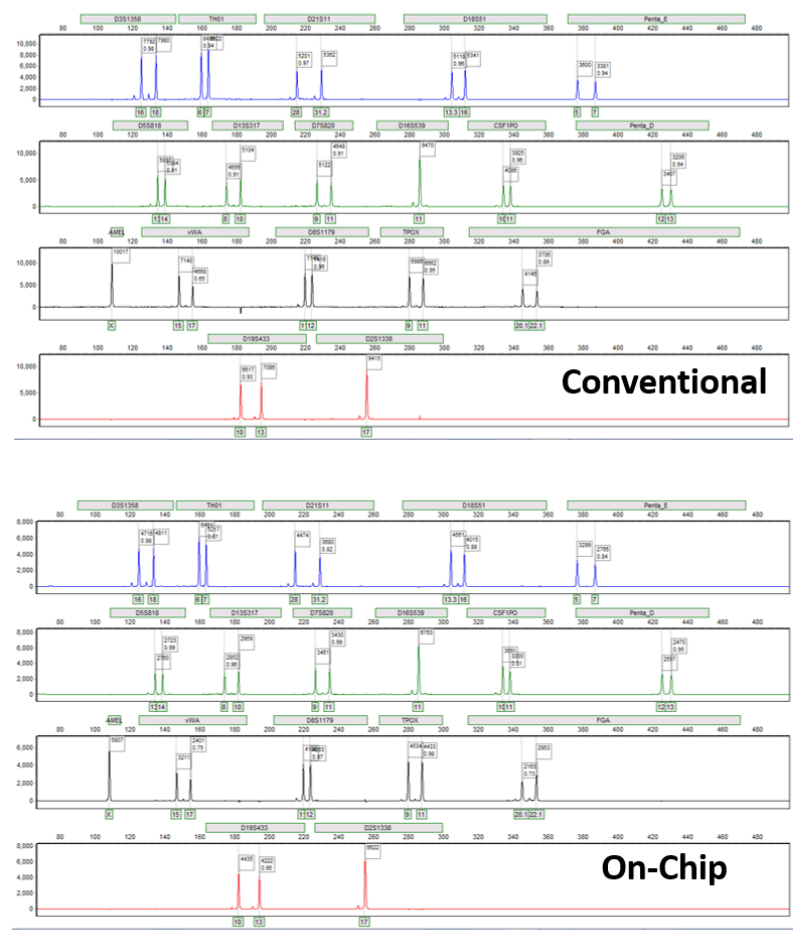

B
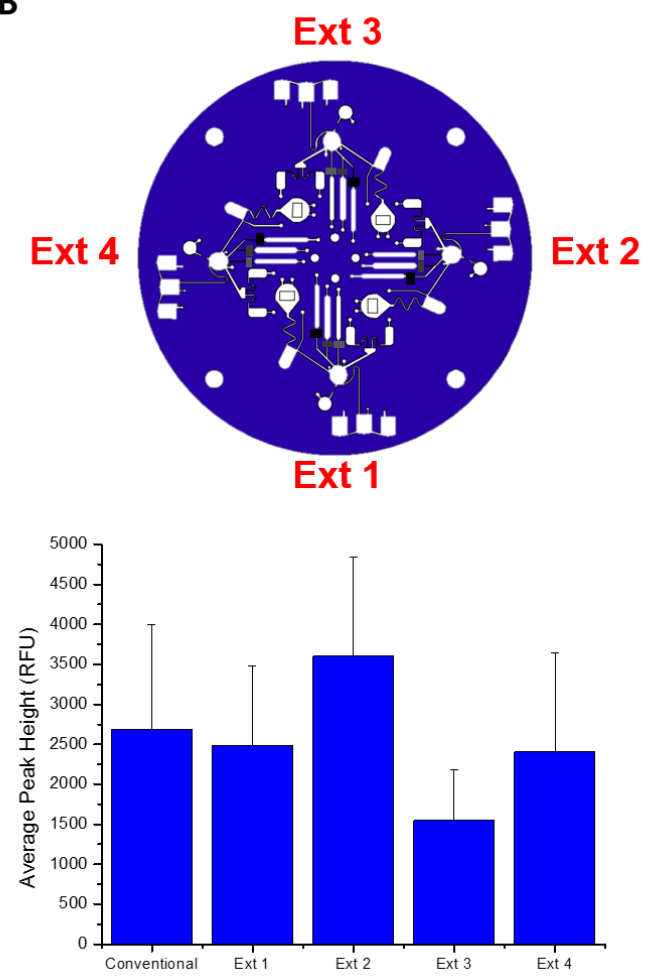

Figure 13: Integrated Lysis and Extraction Analysis. (A) STR comparison of an on-chip integrated lysis and extraction to a conventional method. (B) Labeled lysisdSPE microdevice for a multiplexed lysis-dSPE extraction. A peak height comparison is shown for the STR profiles obtained from four separate buccal swab cuttings as well along with a conventional lysis and extraction as a positive control.

processed samples provided full STR profiles from $2 \mu \mathrm{L}$ of the extracted DNA which were concordant with an off-chip conventional lysis and Qiagen extraction. Slight variability observed in the STR profile was due to the use of different swab cuttings that contain different amounts of buccal cells.

Many advantages of an integrated dSPE device extraction method are outlined in Table 2 and were compared to advantages on a commonly used biorobotic EZ1 instrument. Using the PeT device allows for decreased sample handling time per extraction with a significant 14 -fold decrease in reagent use. In addition, the cost per sample decreases 18 -fold to $\$ 0.25 /$ sample, as the user does 
Table 2: Comparison of dSPE Microdevice to a Conventional Qiagen EZ1 Biorobot

\begin{tabular}{lcc} 
& PeT $\boldsymbol{\mu d e v i c e}$ & Qiagen EZ1 \\
\hline Lysis Volume and Incubation Time & $14 \mu \mathrm{L}, 10$ minutes & $200 \mu \mathrm{L}, 30+$ min \\
Necessary Starting Volume for Extraction & $2 \mu \mathrm{L}$ & $200 \mu \mathrm{L}$ \\
Elution Volume & $6 \mu \mathrm{L}$ & $50 \mu \mathrm{L}$ \\
Cost/Sample & $<\$ 0.50$ & $\$ 9.00$ \\
Time Per Extraction (min) & 20 & 20 \\
Portability & Yes & No \\
Plexing & Single-use & Single-use \\
Sample Multiplexing & Up to 4 samples & Up to 6 samples \\
& & \\
\hline
\end{tabular}

not need to use multiple disposable tubes and cartridges to perform a DNA extraction. The largest advantage may be the portability of the PeT device, which could allow users to use the device in the field. Currently the dSPE device can run 4 samples per chip but there is the possibility for increased throughput by stacking of multiple dSPE devices.

\subsection{Discussion}

Centrifugal microfluidic platforms can be used for the automation and miniaturization of complicated chemistry steps, such as DNA extraction. In this work, we developed a centrifugal microfluidic device with full passive valving to enable automated and inexpensive DNA sample lysis and DNA purification. Fabrication of these PeT devices are cost-efficient due to a simple 'print, cut, laminate' procedure which drive the cost down to approximately $\$ 0.25 /$ sample. This is significantly less expensive than current biorobotic systems ${ }^{25}$ which benefits all forensic laboratories. Comparable STR profiles to a conventional system demonstrates that system processing can also be miniaturized to a hand-held system without compromising the quality of the data. 
The ability to integrate multiple chemical steps on one device indicate that there is an opportunity towards full automation without complex external equipment for fluidic control. Architectural improvements to the extraction domain, such as the addition of a resistive valve, provided improved automation, with reduced hands-on manipulation of the device, and a lower risk for contamination. Our extraction validation data suggests that not only can the system extract DNA with high purity, but also can accommodate samples containing DNA concentrations spanning a minimum of 3 orders of magnitude. Unlike other extraction systems, DNA is processed with centrifugal fluid flow and passive valving, avoiding the use of bulky syringe pumps or external valve actuators which is commonly used in other previously reported extraction systems ${ }^{13}$, 26,27 .

A unique feature in the lysis domain is the calibrated rectangular cutout to accommodate swab cuttings instead of full swabs, as is usually reported for use in literature. Minimized sample consumption offers the opportunity to save precious forensic samples for possible reruns if necessary. Although not required, the developed microdevice also affords the option for retrieving the swab cutting following a lysis and extraction procedure if needed. The cutout also acts as a normalization method to minimize user-to-user variability in swab cuttings and for maximum DNA recovery. When user variability was tested, there was no significant difference between the DNA recovery on four different swab samples.

A large advantage of the integrated system is the ability for on-board lysis and extraction of all samples simultaneously. A single hotplate can be used for effective lysis of all four samples, which is then transferred to the dSPE system for transfer of an aliquot of the lysate to the extraction domain, and then purification of the DNA. We recognize that the integrated dSPE system would need further optimization and integration to be fully automated. For example, building in resistive 
heaters underneath the lysis chambers can allow sample lysis on the spin system directly. Electromagnets would also need to be engineered into the spin system to allow for completely hands-free extraction of the sample. Despite these, this system is a significant step towards the development of a total analysis system.

\subsection{Conclusions}

With an improved PeT DNA extraction design for increased automation, we demonstrated successful DNA purification from dried reference buccal swab samples for the first time. A modified lysis method was validated on a Qiagen EZ1 instrument and demonstrated comparable DNA recovery and STR profiles to a conventional biorobotic method from a $14 \mu \mathrm{L}$ lysis volume and 10 minute incubation. Upon examining the PeT dSPE device materials, we did not find that the materials effected downstream PCR and on-chip mixing did not induce DNA shearing. Using on-chip dSPE extractions with the modified lysis method, strong STR profiles could be obtained from buccal swab samples containing between $30 \mathrm{pg} / \mu \mathrm{L}$ up to $20 \mathrm{ng} / \mu \mathrm{L}$.

We also explored the feasibility of integrating sample lysis with extraction from dried buccal cuttings on a PeT device. An enclosed PeT device with a lysis time of 10 minutes provided sufficient DNA for full STR profiles with a $100 \%$ correlation to control lysis samples. Although not fully optimized for direct integration with dSPE, this work provides a significant step forward towards developing an integrated automated system that is advantageous with forensic DNA applications.

\subsection{References}

1. J. Butler, Advanced Topics in Forensic DNA Typing, Academic Press, 2011.

2. C. W. Price, D. C. Leslie and J. P. Landers, Lab on a Chip, 2009, 9, 2484-2494. 
3. M. COLPAN and D. RIESNER, 1983.

4. D. Riesner and M. Colpan, Journal, 1987.

5. B. Vogelstein and D. Gillespie, Proceedings of the National Academy of Sciences of the United States of America, 1979, 76, 615-619.

6. J. Ducree, S. Haeberle, S. Lutz, S. Pausch, F. von Stetten and R. Zengerle, Journal of Micromechanics and Microengineering, 2007, 17, S103-S115.

7. R. Gorkin, J. Park, J. Siegrist, M. Amasia, B. S. Lee, J. M. Park, J. Kim, H. Kim, M. Madou and Y. K. Cho, Lab on a Chip, 2010, 10, 1758-1773.

8. J. Siegrist, R. Gorkin, M. Bastien, G. Stewart, R. Peytavi, H. Kido, M. Bergeron and M. Madou, Lab on a Chip, 2010, 10, 363-371.

9. D. E. W. Patabadige, S. Jia, J. Sibbitts, J. Sadeghi, K. Sellens and C. T. Culbertson, Analytical Chemistry, 2016, 88, 320-338.

10. D. R. Reyes, D. Iossifidis, P.-A. Auroux and A. Manz, Analytical Chemistry, 2002, 74, 2623-2636.

11. K. J. Shaw, D. A. Joyce, P. T. Docker, C. E. Dyer, G. M. Greenway, J. Greenman and S. J. Haswell, Lab on a Chip, 2011, 11, 443-448.

12. D. Le Roux, B. E. Root, J. A. Hickey, O. N. Scott, A. Tsuei, J. Li, D. J. Saul, L. Chassagne, J. P. Landers and P. de Mazancourt, Lab on a Chip, 2014, 14, 4415-4425.

13. J. Yang, C. Brooks, M. D. Estes, C. M. Hurth and F. Zenhausern, Forensic Science International: Genetics, 2014, 8, 147-158.

14. B. L. Thompson, Y. Ouyang, G. R. M. Duarte, E. Carrilho, S. T. Krauss and J. P. Landers, Nat. Protocols, 2015, 10, 875-886. 
15. G. R. M. Duarte, C. W. Price, B. H. Augustine, E. Carrilho and J. P. Landers, Analytical Chemistry, 2011, 83, 5182-5189.

16. G. R. M. Duarte, C. W. Price, J. L. Littlewood, D. M. Haverstick, J. P. Ferrance, E. Carrilho and J. P. Landers, Analyst, 2010, 135, 531-537.

17. D. Kimes and M. Tamir, Crime Laboratory Digest, 1985, 12, 32-33.

18. J. V. Norris, K. Manning, S. J. Linke, J. P. Ferrance and J. P. Landers, Journal of Forensic Sciences, 2007, 52, 800-805.

19. K. R. Jackson, E. Kamano, J. DuVall, and J. P. Landers, In Progress.

20. J. B. B. K. R. Jackson, M. Meija, D. L. Mills, D. M. Haverstick, K. E. Olson, R. Aranda , G. T. Garner, E. Carrilho and J. P. Landers, Analytica Chimica Acta, Submitted.

21. Y. W. Ouyang, S. B. Wang, J. Y. Li, P. S. Riehl, M. Begley and J. P. Landers, Lab on a Chip, 2013, 13, 1762-1771.

22. Qiagen, EZ1® DNA Investigator® Handbook, file://C:/Users/kjacks07/Downloads/EZ1DNA-Investigator-Handbook\%20(1).pdf).

23. Promega, Maxwell@ 16 DNA Purification Kits Technical Manual, https://www.promega.com/ /media/files/resources/protocols/technical\%20manuals/0/ma xwell\%2016\%20dna\%20purification\%20kits\%20protocol.pdf).

24. T. F. Scientific, MagMAX ${ }^{\mathrm{TM}}$ Total Nucleic Acid Isolation Kit, https://www.thermofisher.com/order/catalog/product/AM1840?ICID=search-product).

25. Qiagen, QIAGEN® Price List 2013-2014 for Sample and Assay Technologies, file:///C:/Users/kjacks07/Downloads/US\%20Pricelist\%202013-2014.pdf).

26. W. P. Gan, B. Zhuang, P. F. Zhang, J. P. Han, C. X. Li and P. Liu, Lab on a Chip, 2014, 14, 3719-3728. 
27. W. Cho, J.-H. Maeng, Y. Ahn and S. Y. Hwang, Electrophoresis, 2013, 34, 2531-2537. 


\section{Conclusions and Future Directions}

\subsection{Overarching Conclusions and Future Directions}

\subsubsection{Sample Preparation}

This dissertation described methods for rapid and cost-effective nucleic acid processing for clinical and/or forensic samples. This is critical, as presumptive testing of DNA in unknown stains is valuable and can identify contextual clues to help in criminal investigations or for clinical diagnosis. Unfortunately, most laboratories are still reliant on archaic technology that lacks specificity and is prone to false positives. Recent attempts to solve this problem have led to the development of methods that are significantly more time consuming and expensive. Here, two optical methods are described that are competitive with newly developed methods, but simple and cost-effective.

Loop-mediated isothermal amplification (LAMP) is a simple and isothermal amplification method that was critical for the development of rapid detection methods of target analytes. In Chapter 2, LAMP was combined with a novel fluorescent-free bead detection method (high affinity aggregation) for sequence-specific detection of target DNA or RNA. There were three primary parts that were optimized for successful detection: (i) biotin labeling of target sequences; (ii) biotin-streptavidin bead aggregation; and (iii) optical detection. The biotin labeling of two LAMP primers was found to selectively transfer biotin to sequences of interest. Using streptavidin particles and a rotating magnetic field, only sequences containing biotin could trigger bead aggregation. This controlled aggregation became known as high affinity aggregation, or HAA. Following optimization studies, the HAA method had a large detection bandwidth which is almost a 20-fold improvement over current bead detection of loop-mediated amplification amplicons. The image algorithm, used for analysis of the bead aggregates, was altered to provide the greatest 
distinction between positive and negative control samples. Using these optimized parameters, multiple applications for HAA were reported and confirmed the ability to distinguish any DNA or RNA sequence with a single base mutation. The high specificity of the LAMP method combined with the biotin-streptavidin binding eliminated any false aggregation that could occur for accurate detection of a target of interest.

Real-time studies of HAA revealed possible quantitative measurement capability, but was not explored further. It would be beneficial to see whether HAA could be used as a fluorescentfree DNA quantitation method if this work was continued. All primers used in HAA were saltfree due to the increased cost of biotin labeling, however, the salt-free primers may not be pure enough to accurately estimate the DNA concentration in a given sample. Higher purity primers may provide a more reproducible estimation of the quantity of DNA and which would improve on a previously developed LAMP method demonstrating accurate quantitation of DNA using the Amelogenin locus ${ }^{1}$. Accurate quantitation of Amelogenin has already been demonstrated via a turbidimeter ${ }^{1}$, therefore, it is feasible to provide quantitative detection of target sequences via HAA on an inexpensive, fluorescent-free platform.

HAA could also be further improved with a newly discovered OmniAmp® polymerase that is reportedly faster than traditional Bst polymerase used in LAMP reactions ${ }^{2}$. This could generate even more rapid amplification and detection in less time, with increased thermal stability of a reaction above $70{ }^{\circ} \mathrm{C}$. Studies show increased sensitivity with the new polymerase down to 1 copy of DNA as well which can amplify within 20 minutes $^{2}$. This would be advantageous if a sample contains trace amounts of nucleic material.

Another improvement to the HAA platform would be to adapt the current system to a microdevice platform. LAMP amplification has been done in a PeT device and does not inhibit 
amplification. However, the bead detection step will be challenging, as preliminary experiments observed particle aggregation in samples that did not contain nucleic material. This is most likely due to non-specific aggregation of the streptavidin particles caused by the biotin-labelled primers. As the primer concentration is necessary for LAMP amplification, this cannot be changed. An alternative method would be to explore methods for agitating the microdevice, as was done in the normal HAA platform. This would break apart any weak non-specific binding species, providing more accurate analysis. Once an agitation method is found to work, the sample addition, amplification, could be integrated on into one device. If combined with the increased purity LAMP primers, then it may be possible to provide quantitative detection on a microdevice platform.

Chapter 3 discussed using LAMP and optical detection via a smart phone for the detection of a panel of 5 body fluids: blood, vaginal fluid, saliva, normal semen, and azoospermatic semen samples. Custom LAMP primers were designed for each body fluid and optimized to operate at the same temperature. Unknown samples containing any combination of the described body fluids above could be analyzed simultaneously with the development of a universal sample processing method. Detection was simplified with the addition of a metal indicator dye to each LAMP reaction that offered a visual colorimetric response following successful LAMP amplification. This color change can easily be captured by a cell phone and analyzed via image analysis. To provide for high sample efficiency, this method was adapted for a 96-well plate format which can process up to 23 samples simultaneously. Difficult samples dried on denim, which could not otherwise be tested via colorimetric testing, was successfully detected within 15 minutes using the LAMP method. This is a significant advancement over archaic colorimetric testing for body fluids, providing increased sensitivity without compromising the simplicity of the method. Unlike other 
body fluid methods that utilize complicated sample preparation or expensive instrumentation, this LAMP method only needs a hot plate and a cell phone to run samples.

The body fluid identification (BFI) method is only as discriminatory as the mRNA target, which makes it difficult to expand to other body fluids with markers found in multiple fluids, including menstrual blood and sweat. However, micro RNA or unique bacteria found in these other body fluids have been explored for potential unique markers. Several markers have been proposed $^{3-6}$ which could be exploited with LAMP and optimized to operate at the same conditions as the blood, vaginal fluid, semen, and saliva assays. Since DNA and RNA co-purifies in a DNA extraction, the universal sample method may not be compromised if both RNA and DNA are needed for BFI.

To make this method more efficient, it would be worth exploring a different chemistry for sample lysis and extraction. ZyGEM technology liberates the nucleic acid in one step from the raw sample of interest and has been shown to be compatible with a custom LAMP assay ${ }^{7}$. The compatibility of ZyGEM chemistry with the traditional LAMP kit was tested in preliminary experiments and all resulted in reproducible failed LAMP assays. Further experimentation revealed that even when ZyGEM buffer was added as a sample (with control RNA added separately), the LAMP assay failed. The traditional LAMP kit was not tested, so it is unclear whether the buffer was inhibitory to the reverse transcriptase, or to the Bst polymerase. Rather than changing the chemistry of the LAMP assay, it would be simpler to change the chemical components associated with ZyGEM liberation. These modifications include changing the concentration of the buffer, the $\mathrm{pH}$, etc. If the ZyGEM chemistry modifications are successful, sample preparation time could be reduced by at least $50 \%$. One downside to this approach would be the required dilution of the sample RNA during ZyGEM sample preparation, which will 
decrease the sensitivity of the overall system. Instead of providing purified RNA (and co-purified DNA) in $30 \mu \mathrm{L}$ by Qiagen technology, the sample would now process in $50-100 \mu \mathrm{L}$. This could be a significant problem if the user is handling samples with trace amounts of nucleic material.

Another improvement of the BFI system is further automation, which would involve the placement of a custom isothermal heater in the top of the photo box that is used to image the entire plate. Since the 96-well plate will be in a fixed position, the location of the wells could be easily estimated and used for automated software development based on hue analysis. This could minimize any user involvement and provide rapid sample analysis. One engineering challenge for the automated system would be temperature control, as the data from Chapter 3 showed the significant change in fluid-specificity within $1^{\circ} \mathrm{C}$. However, thermocyclers currently work via a peltier system, which could easily be integrated into an automated system.

\subsubsection{DNA Purification}

DNA purification is presented in Chapter $\mathbf{4}$ and Chapter 5, which describe the adaptation of dSPE to a microdevice and work towards automation and integration. DNA extraction has been adapted to a variety of microfluidic platforms, but many of these methods target large sample volumes that provide no obvious advantage over conventional methods or complicate devices with bulky external syringe pumps or valving hardware. A PeT microdevice purified blood from only $0.6 \mu \mathrm{L}$ of whole blood, but was limited by the number of manual steps ${ }^{8}$. In an effort to increase the automation of this method, Chapter 4 describes the development of a rotationally-driven PeT device for purification of human genomic DNA from whole blood samples. Increased automation provided the opportunity for multiplexing the dSPE process. Many optimization studies were run to determine the architectural design for dSPE as well as optimal binding/elution of DNA and 
silica particles and the speeds and time for efficient washing. Fluidic control was a challenge even with the use of passive valves, therefore, a novel 'tape valve' was developed. These tape valves were either normally open or normally closed valves and, when closed, had a measured burst pressure of $9.17 \pm 1.67 \times 10^{-3} \mathrm{~Hz}$. Once optimized, the dSPE device provided PCR-ready DNA which was amplified in a singleplex $\beta$-globin reaction and, for the first time, a more complicated multiplexed STR reaction. An extraction efficiency of $44 \%$ was achieved, which is a $13 \%$ improvement over the bacterial extraction method previously described.

In Chapter 5, architectural improvements were made to the dSPE disc described in Chapter 4, which further increased the automation for dSPE. All manual valve manipulation was eliminated with the addition of a resistive valve. In addition, DNA extractions were catered to buccal swab cuttings which are more relevant forensic samples. These extractions could provide equivalent STR profiles to a biorobotic instrument for samples containing between $300 \mathrm{pg}$ total DNA up to $100 \mathrm{ng}$. Sample lysis was integrated upstream of the dSPE extraction architecture, which allowed for sample-to-PCR ready DNA for four samples from just $14 \mu \mathrm{L}$ of lysate. STR profiles from four simultaneous lysis and extractions correlated to a conventional method. This miniaturization proved that comparable STR profiles could be obtained with a single closed system, which is a significant step towards the development of a total analysis system.

The obvious next step for the technology described in Chapters 4-5 is continued automation of the system. This involves the integration of electromagnets to keep the silica beads in place as the disc is spinning, which is challenging from an engineering standpoint. Currently, Neodymium magnets are manually placed on the system, which could be easily exchanged for electromagnets. However, the electromagnets that are comparable in strength to the Neodymium magnets are bulky in size and require a separate power supply. This compromises the portability 
of the system and will be difficult to find space for all of the required components. Since power needs to be sent to the electromagnets as the disc spins, a slip ring will also need to be integrated on the system. The only method of doing this would be to change the current position of the sample motor, which means re-engineering of the entire system.

Once the automation of dSPE is finished, other processes, such as PCR could be placed downstream of the extraction chamber to work towards a fully automated system. Chemically, this can be challenging, as the toner particulates are inhibitory to PCR. However, chemical additives, such as bovine serum albumin (BSA) can counteract this inhibition for increased PCR efficiency. This process may also be difficult from an engineering perspective, as PCR requires careful temperature monitoring. The most efficient method would be to integrate an on-board Peltier system which could clamp the disc in place and provide accurate temperature profiles. However, integrating a Peltier system on the current dSPE system will not be an easy task.

Since RNA co-extracts with DNA with the silica chemistry, it would be interesting to see how much RNA could be recovered from a given sample. This dSPE device, if efficient with RNA extraction, could be used in tandem with the body fluid identification for possible full automation of the BFI process. Based on preliminary studies, switching the silica beads to nanoparticles would increase the overall extraction efficiency to be between $70-80 \%$. However, silica nanoparticles are difficult to control with a magnetic and will inhibit downstream PCR. Other coatings, such as chitosan could be used instead on the nanoparticles to provide increased extraction efficiency without worrying about PCR inhibition. 


\subsection{Summation}

The work described in this thesis focuses on advancing technology associated with sample preparation and DNA purification. The identification of DNA from unknown material was shown in two separate methods via an isothermal amplification. HAA provides sequence-specific quantitative measuring capability of DNA in unknown samples whereas body fluid identification offers a simple colorimetric analysis. The next steps in DNA processing were explored in the following chapters to describe the adaptation of sample lysis and DNA extraction to a centrifugal microfluidic platform. The innovative sample processing methods described in this thesis benefit clinical and/or forensic laboratories by providing accurate, highly specific results from semiautomated methods. Future directions include further work towards full automation and of other similar applications of interest to clinical and/or forensic laboratories.

\subsection{References}

1. R. W. Allen and V. M. Fuller, Journal of forensic sciences, 2006, 51, 76-81.

2. Y. Chander, J. Koelbl, J. Puckett, M. J. Moser, A. J. Klingele, M. R. Liles, A. Carrias, D. A. Mead and T. W. Schoenfeld, Frontiers in Microbiology, 2014, 5.

3. X. Luo, Z. Li, D. Peng, L. Wang, L. Zhang and W. Liang, Forensic Science International: Genetics Supplement Series, 2015, 5, e630-e632.

4. E. Hanson, H. Lubenow and J. Ballantyne, Forensic Science International: Genetics Supplement Series, 2009, 2, 503-504.

5. T. Akutsu, H. Motani, K. Watanabe, H. Iwase and K. Sakurada, Legal Medicine, 2012, 14, 160162.

6. C. C. Benschop, F. C. Quaak, M. E. Boon, T. Sijen and I. Kuiper, International journal of legal medicine, 2012, 126, 303-310. 
7. T. Segawa, Y. Kobayashi, Y. Sase, T. Itou, M. Suzuki, T. Endoh, T. Nakanishi and T. Sakai, Journal of virological methods, 2014, 201, 31-37.

8. G. R. M. Duarte, C. W. Price, B. H. Augustine, E. Carrilho and J. P. Landers, Analytical Chemistry, 2011, 83, 5182-5189. 